\title{
Commercial Motor Vehicle Driving Simulator Validation Study: Phase II
}

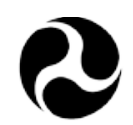

U.S. Department of Transportation 


\section{FOREWORD}

The work performed under this project included multiple research efforts designed to investigate the use of simulators in training commercial motor vehicle drivers. The topics addressed in this project include the effectiveness of entry-level training (including simulation-based training) for commercial licensing testing and the effectiveness of different training methods in terms of licensing skills test scores and driving records post-licensure. As part of this project, several simulated advanced scenarios (e.g., tanker trailer, tire blowout) are showcased as well. A cost analysis of different training methods was performed as part of this project, along with a case study of existing uses of commercial vehicle driving simulators. This report documents the method, results, and conclusions of these efforts.

\section{NOTICE}

This document is disseminated under the sponsorship of the U.S. Department of Transportation in the interest of information exchange. The United States Government assumes no liability for its contents or the use thereof.

The contents of this Report reflect the views of the contractor, who is responsible for the accuracy of the data presented herein. The contents do not necessarily reflect the official policy of the Department of Transportation.

This Report does not constitute a standard, specification, or regulation.

The United States Government does not endorse products or manufacturers named herein. Trade or manufacturers' names appear herein only because they are considered essential to the objective of this document. 
Technical Report Documentation Page—Form DOT F 1700.7 (8-72)

\begin{tabular}{|c|c|c|c|c|}
\hline $\begin{array}{l}\text { 1. Report No. } \\
\text { FMCSA-RRR-11-014 }\end{array}$ & \multicolumn{2}{|c|}{ 2. Government Accession No. } & \multicolumn{2}{|c|}{ 3. Recipient's Catalog No. } \\
\hline \multirow{2}{*}{\multicolumn{3}{|c|}{$\begin{array}{l}\text { 4. Title and Subtitle } \\
\text { COMMERCIAL MOTOR VEHICLE DRIVING SIMULATOR } \\
\text { VALIDATION STUDY: PHASE II }\end{array}$}} & \multicolumn{2}{|l|}{$\begin{array}{l}\text { 5. Report Date } \\
\text { April } 2011\end{array}$} \\
\hline & & & \multicolumn{2}{|c|}{ 6. Performing Organization Code } \\
\hline \multicolumn{3}{|c|}{$\begin{array}{l}\text { 7. Author(s) } \\
\text { Justin F. Morgan, Scott A. Tidwell, Alejandra Medina, Myra Blanco, } \\
\text { Jeffrey S. Hickman, and Richard J. Hanowski }\end{array}$} & \multicolumn{2}{|c|}{ 8. Performing Organization Report No. } \\
\hline \multirow{2}{*}{\multicolumn{3}{|c|}{$\begin{array}{l}\text { 9. Performing Organization Name and Address } \\
\text { Center for Truck and Bus Safety } \\
\text { Virginia Tech Transportation Institute } \\
3500 \text { Transportation Research Plaza (0536) } \\
\text { Blacksburg, VA } 24061\end{array}$}} & \multicolumn{2}{|c|}{ 10. Work Unit No. (TRAIS) } \\
\hline & & & \multicolumn{2}{|c|}{$\begin{array}{l}\text { 11. Contract or Grant No. } \\
\text { DTNH22-05-D-01019 Task Order \# } 9\end{array}$} \\
\hline \multirow{2}{*}{\multicolumn{3}{|c|}{$\begin{array}{l}\text { 12. Sponsoring Agency Name and Address } \\
\text { U.S. Department of Transportation } \\
\text { Federal Motor Carrier Safety Administration } \\
1200 \text { New Jersey Ave. SE } \\
\text { Washington, DC } 20590\end{array}$}} & \multicolumn{2}{|c|}{$\begin{array}{l}\text { 13. Type of Report and Period Covered } \\
\text { Final Report, } \\
\text { August 2006-March } 2010\end{array}$} \\
\hline & & & \multicolumn{2}{|c|}{$\begin{array}{l}\text { 14. Sponsoring Agency Code } \\
\text { FMCSA }\end{array}$} \\
\hline \multicolumn{5}{|c|}{$\begin{array}{l}\text { 15. Supplementary Notes } \\
\text { This report was sponsored by the Federal Motor Carrier Safety Administration. The Contracting Officer's } \\
\text { Technical Representative: Olu Ajayi }\end{array}$} \\
\hline \multicolumn{5}{|l|}{ 16. Abstract } \\
\hline \multicolumn{5}{|c|}{$\begin{array}{l}\text { This study examined the effectiveness of a driving simulator for entry-level commercial motor vehicle (CMV) driver } \\
\text { training and testing. Four training groups of } 107 \text { individuals (conventional } 8 \text {-week certified course, conventional } 8 \text { - } \\
\text { week certified course with } 60 \text { percent of driving in a simulator, informal training with friends/relatives, and } \\
\text { commercial's driver license [CDL] test-focused short courses) were followed from training into employment. There } \\
\text { were no group differences in Division of Motor Vehicles (DMV) road tests. There were group differences in DMV } \\
\text { range tests and validated real truck and simulator re-creations of DMV road and range tests. Conventional and } \\
\text { simulator groups generally scored higher than informal and CDL test-focused participants. A 4-month follow-up } \\
\text { after being hired as a CDL driver indicated no differences in performance, safety, self- or supervisory-ratings. } \\
\text { Findings support the use of CMV driving simulator-based training, but simulator-based testing does not appear to } \\
\text { be feasible at this point. Cost analysis indicated simulator training using the study simulator was } \$ 35 / \text { participant } \\
\text { less expensive than conventional training. The simulator was examined in a demonstration of extreme conditions } \\
\text { and emergency maneuvers under different vehicle configurations with } 48 \text { other drivers. Also provided is a case } \\
\text { study of existing implementations of CMV simulator training, indicating benefits, drawbacks, and drivers' overall } \\
\text { opinions. }\end{array}$} \\
\hline \multicolumn{2}{|c|}{$\begin{array}{l}\text { 17. Key Words } \\
\text { advanced capabilities, CDL, CMV, commercial driver } \\
\text { license, commercial motor vehicle, driver training, } \\
\text { entry-level, forward transfer, longitudinal evaluation } \\
\text { simulator, tanker, training, tractor-trailer } \\
\end{array}$} & \multicolumn{3}{|c|}{$\begin{array}{l}\text { 18. Distribution Statement } \\
\text { No restrictions }\end{array}$} \\
\hline $\begin{array}{l}\text { 19. Security Classif. (of this } \\
\text { Unclassified }\end{array}$ & $\begin{array}{l}\text { 20. Security Clas } \\
\text { Unclassified }\end{array}$ & this page) & $\begin{array}{l}\text { 21. No. of Pages } \\
272\end{array}$ & 22. Price \\
\hline
\end{tabular}

Form DOT F 1700.7 (8-72)

Reproduction of completed page authorized. 


\section{SI* (MODERN METRIC) CONVERSION FACTORS}

\begin{tabular}{|c|c|c|c|c|}
\hline Symbol & When You Know & Multiply By & To Find & Symbol \\
\hline \multicolumn{5}{|c|}{ LENGTH } \\
\hline In & Inches & 25.4 & Millimeters & $\mathrm{mm}$ \\
\hline $\mathrm{Ft}$ & Feet & 0.305 & Meters & $\mathrm{m}$ \\
\hline Yd & Yards & 0.914 & Meters & $\mathrm{m}$ \\
\hline Mi & Miles & 1.61 & Kilometers & $\mathrm{km}$ \\
\hline \multicolumn{5}{|c|}{ AREA } \\
\hline $\mathrm{in}^{2}$ & square inches & 645.2 & square millimeters & $\mathrm{mm}^{2}$ \\
\hline $\mathrm{ft}^{2}$ & square feet & 0.093 & square meters & $\mathrm{m}^{2}$ \\
\hline$y d^{2}$ & square yards & 0.836 & square meters & $\mathrm{m}^{2}$ \\
\hline $\mathrm{Ac}$ & Acres & 0.405 & Hectares & ha \\
\hline \multirow[t]{2}{*}{$\mathrm{mi}^{2}$} & square miles & 2.59 & square kilometers & $\mathrm{km}^{2}$ \\
\hline & & VOLUME & $\begin{array}{l}\text { Note: Volumes greater than } \\
1000 \mathrm{~L} \text { shall be shown in } \mathrm{m}^{3}\end{array}$ & \\
\hline fl oz & fluid ounces & 29.57 & Milliliters & $\mathrm{mL}$ \\
\hline Gal & Gallons & 3.785 & Liters & $\mathrm{L}$ \\
\hline $\mathrm{ft}^{3}$ & cubic feet & 0.028 & cubic meters & $\mathrm{m}^{3}$ \\
\hline$y d^{3}$ & cubic yards & 0.765 & cubic meters & $\mathrm{m}^{3}$ \\
\hline \multicolumn{5}{|c|}{ MASS } \\
\hline $\mathrm{Oz}$ & Ounces & 28.35 & Grams & $g$ \\
\hline $\mathrm{Lb}$ & Pounds & 0.454 & Kilograms & $\mathrm{kg}$ \\
\hline $\mathrm{T}$ & short tons (2000 lb) & 0.907 & megagrams (or "metric ton") & $\mathrm{Mg}$ (or "t") \\
\hline & & TEMPERATURE & Temperature is in exact degrees & \\
\hline${ }^{\circ} \mathrm{F}$ & Fahrenheit & $\begin{array}{l}5 \times(F-32) \div 9 \\
\text { or }(F-32) \div 1.8\end{array}$ & Celsius & ${ }^{\circ} \mathrm{C}$ \\
\hline & & ILLUMINATION & & \\
\hline $\mathrm{Fc}$ & foot-candles & 10.76 & Lux & Lx \\
\hline $\mathrm{Fl}$ & foot-Lamberts & 3.426 & candela/m² & $\mathrm{cd} / \mathrm{m}^{2}$ \\
\hline \multicolumn{5}{|c|}{ Force and Pressure or Stress } \\
\hline Lbf & poundforce & 4.45 & Newtons & $\mathrm{N}$ \\
\hline lbf/in ${ }^{2}$ & poundforce per square inch & 6.89 & Kilopascals & $\mathrm{kPa}$ \\
\hline \multicolumn{5}{|c|}{ Table of APPROXIMATE CONVERSIONS FROM SI UNITS } \\
\hline Symbol & When You Know & Multiply By & To Find & Symbol \\
\hline \multicolumn{5}{|c|}{ LENGTH } \\
\hline $\mathrm{Mm}$ & millimeters & 0.039 & Inches & In \\
\hline M & meters & 3.28 & Feet & $\mathrm{Ft}$ \\
\hline M & meters & 1.09 & Yards & Yd \\
\hline $\mathrm{Km}$ & kilometers & 0.621 & Miles & $\mathrm{Mi}$ \\
\hline \multicolumn{5}{|c|}{ AREA } \\
\hline $\mathrm{mm}^{2}$ & square millimeters & 0.0016 & square inches & in $^{2}$ \\
\hline $\mathrm{m}^{2}$ & square meters & 10.764 & square feet & $\mathrm{ft}^{2}$ \\
\hline $\mathrm{m}^{2}$ & square meters & 1.195 & square yards & $\mathrm{yd}^{2}$ \\
\hline $\mathrm{Ha}$ & hectares & 2.47 & Acres & Ac \\
\hline $\mathrm{km}^{2}$ & square kilometers & 0.386 & square miles & $\mathrm{mi}^{2}$ \\
\hline \multicolumn{5}{|c|}{ VOLUME } \\
\hline $\mathrm{mL}$ & milliliters & 0.034 & fluid ounces & $\mathrm{fl} \mathrm{oz}$ \\
\hline $\mathrm{L}$ & liters & 0.264 & Gallons & Gal \\
\hline $\mathrm{m}^{3}$ & cubic meters & 35.314 & cubic feet & $\mathrm{ft}^{3}$ \\
\hline $\mathrm{m}^{3}$ & cubic meters & 1.307 & cubic yards & $\mathrm{yd}^{3}$ \\
\hline \multicolumn{5}{|c|}{ MASS } \\
\hline G & grams & 0.035 & Ounces & $\mathrm{OZ}$ \\
\hline $\mathrm{Kg}$ & kilograms & 2.202 & Pounds & $\mathrm{lb}$ \\
\hline $\mathrm{Mg}$ (or "t") & megagrams (or "metric ton") & 1.103 & short tons (2000 lb) & $\mathrm{T}$ \\
\hline${ }^{\circ} \mathrm{C}$ & Celsius & $\begin{array}{c}\text { TEMPERATURE } \\
1.8 C+32\end{array}$ & $\begin{array}{l}\text { Temperature is in exact degrees } \\
\text { Fahrenheit }\end{array}$ & ${ }^{\circ} \mathrm{F}$ \\
\hline \multicolumn{5}{|c|}{ ILLUMINATION } \\
\hline Lx & $\operatorname{lux}$ & 0.0929 & foot-candles & fc \\
\hline $\mathrm{cd} / \mathrm{m}^{2}$ & candela/m² & 0.2919 & foot-Lamberts & $\mathrm{fl}$ \\
\hline \multicolumn{5}{|c|}{ Force \& Pressure or Stress } \\
\hline $\mathrm{N}$ & newtons & 0.225 & Poundforce & lbf \\
\hline $\mathrm{kPa}$ & Kilopascals & 0.145 & poundforce per square inch & lbf/in² \\
\hline
\end{tabular}

* $\mathrm{SI}$ is the symbol for the International System of Units. Appropriate rounding should be made to comply with Section 4 of ASTM E380. (Revised March 2003, Section 508-accessible version September 2009) 


\section{TABLE OF CONTENTS}

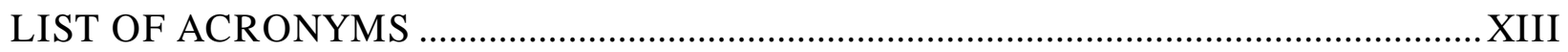

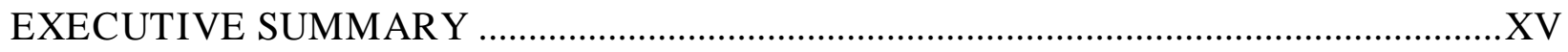

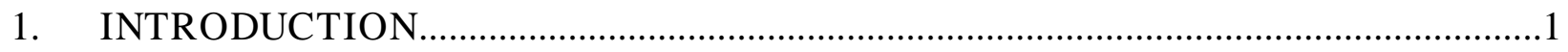

1.1 PROJECT OVERVIEW …………………………………………………………1

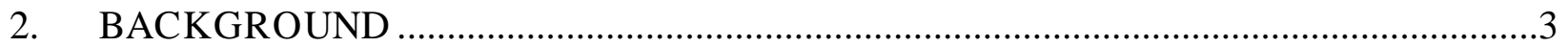

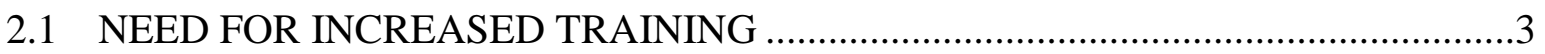

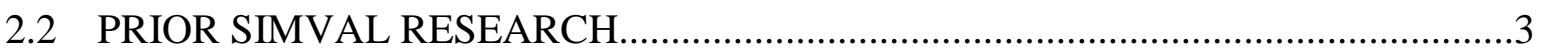

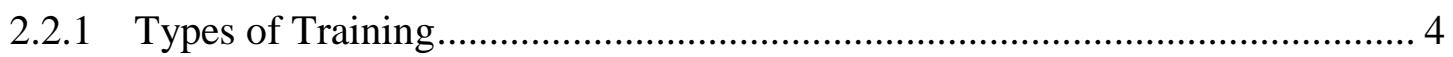

2.2.2 Simulator Based Training Background........................................................... 4

2.2.3 Benefits and Drawbacks to Driving Simulators................................................. 5

2.2.4 Types of Simulators Currently in Use ............................................................ 7

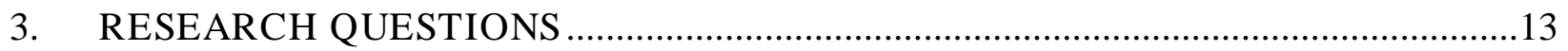

3.1 STUDY OBJECTIVES.....................................................................................13

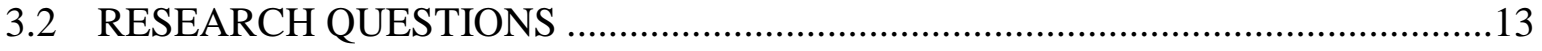

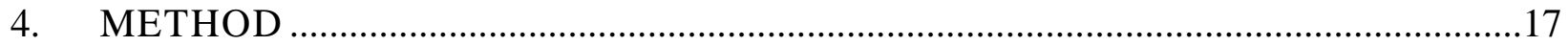

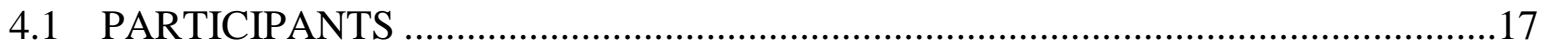

4.1.1 Entry-Level Training Study ………………………................................ 17

4.1.2 Advanced Capabilities Showcase ............................................................. 18

4.1.3 Participant Protections ............................................................................... 20

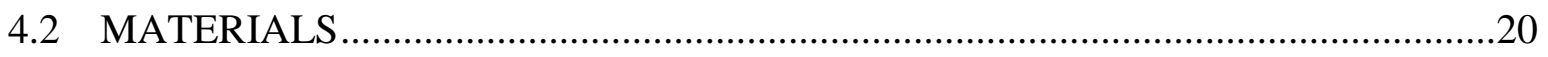

4.2.1 Experimental Venue............................................................................... 20

4.2.2 Entry-Level Training Curriculum ............................................................. 20

4.2.3 Data Acquisition System......................................................................... 22

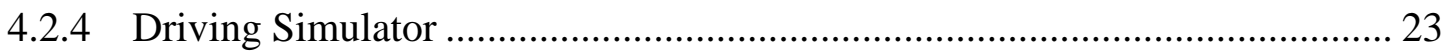

4.2.5 Driving Simulator Scenarios................................................................ 24

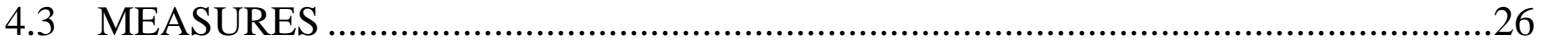

4.3.1 Performance Measures........................................................................ 26

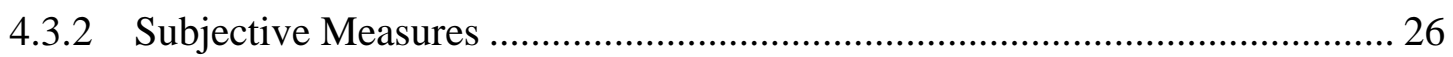

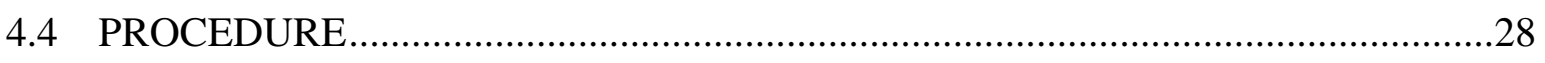

4.4.1 Sample Determination and Power Analysis ................................................... 28

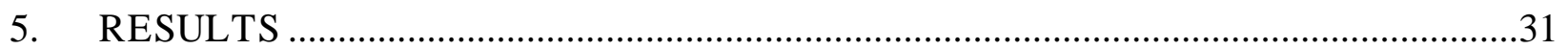


5.1 INTRODUCTION AND OVERVIEW .....

5.2 TOPIC \#1: EFFECTS OF TRAINING METHODS ON CDL AND RELATED TEST PERFORMANCE.

5.2.1 Research Question 1a: Does the training method affect DMV road/range test performance?.

5.3 TOPIC \#2: VALIDITY OF SIMULATION TRAINING AND TESTING ……….......37

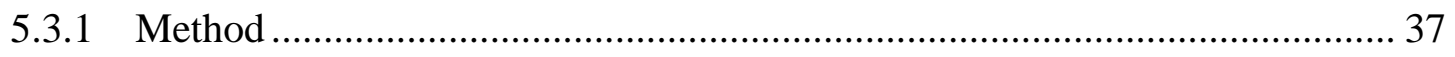

5.3.2 Research Question 2a: Can simulation testing adequately assess road/range

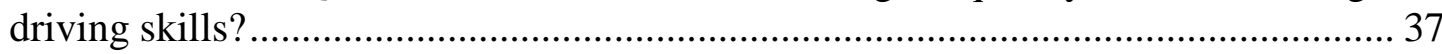

5.3.3 Research Question 2b: What is the correlation between scores from the DTCC road and range tests and simulator road and range tests? ............................................ 45

5.3.4 Research Question 2c: Which road and range test scores (DTCC or Simulated) are more predictive of subsequent DMV road and range tests? ................................... 56

5.3.5 Research Question 2d: What features of the simulator will be most and least effective for participants primarily trained on the simulator? ...................................... 57

5.4 TOPIC \#3: LONGITUDINAL EFFECTS OF TRAINING METHOD ON

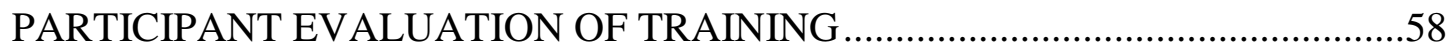

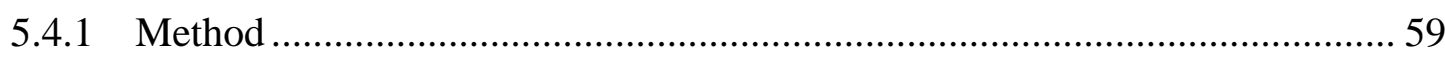

5.4.2 Research Question 3a: Does training method affect the self-ratings of drivers' current performance? 59

5.4.3 Research Question 3b: How does training method affect participant retrospective self-ratings of the training method received in terms of teaching vehicle control, maneuvering skills, and safe operations in comparison to their peers? 60 5.4.4 Research Question 3c: Does training method affect trainee evaluation of training at the end of training and 4 months after being employed as a CDL driver? 61 5.4.5 Research Question 3d: Would participants trained primarily on the simulator deem the simulator skill tests as a valid and fair assessment of their driving skills at the time they completed their training programs?

5.4.6 Research Question 3e: Would participants recommend their method of

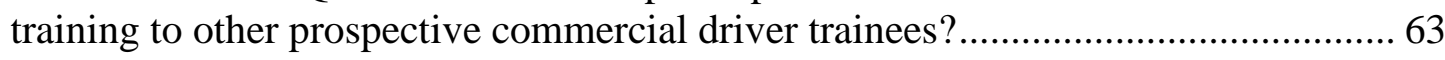

5.4.7 Discussion.

5.5 TOPIC \#4: LONGITUDINAL EFFECTS OF TRAINING METHOD ON DRIVER PERFORMANCE.

5.5.1 Research Question 4a: Does training method affect performance on the simulated road/range and DTCC road/range test during the retest $4-5$ months after they start working as a commercial driver where a CDL is required? Furthermore, does the training method affect the magnitude of change in driving performance 4-5 months after receiving a CDL?

5.6 TOPIC \#5: LONGITUDINAL EFFECTS OF TRAINING METHOD ON SAFETY PERFORMANCE BASED ON DRIVING RECORDS 
5.6.1 Research Question 5a: Does training method affect safety performance on the job (e.g., incident rates, number of violations, number of crashes, supervisor ratings) after 4 and 12 months on the job?

5.7 TOPIC \#6: LONGITUDINAL EFFECTS OF TRAINING METHOD ON SUPERVISOR EVALUATION OF EMPLOYEES

5.7.1 Research Question 6a: Does training method affect supervisors' evaluation of their employees at 4-5 months after being hired and the number of DMV-reported and unreported incidents and violations?

5.8 TOPIC \#7: EXPLORATORY ANALYSIS OF “BIG FIVE” PERSONALITY CORRELATES

5.8.1 Research Question 7a: What is the relationship between the "Big Five" personality correlates (i.e., openness, conscientiousness, extraversion, agreeableness, and neuroticism) and forward transfer of training and longitudinal effects?... 74

5.9 TOPIC \#8: ASSESSING ADVANCED CAPABILITIES OF THE SIMULATOR ...77

5.9.1 Research Question 8a: Are there differences in driver performance between million mile drivers and non-million mile drivers for the following types of advanced capability skills in a simulated environment:.

5.10 TOPIC \#9: PARTICIPANTS’ RATINGS OF THE VARIOUS ASPECTS OF THE ADVANCED CAPABILITIES OF THE SIMULATOR.

5.10.1 Research Question 9a: How do both million-mile and non-million-mile drivers rate the usefulness and realism of the simulator for the presentation of the advanced capabilities?

5.10.2 Research Question 9b: Which emergency maneuvers, driving conditions, or other events provided by the simulator are most important and valuable to drivers, and which are rated as the most realistic by the drivers?

5.10.3 Method

6. COST EFFECTIVENESS ANALYSIS OF ENTRY-LEVEL CMV DRIVER TRAINING

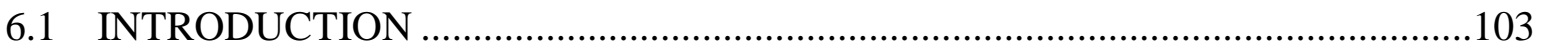

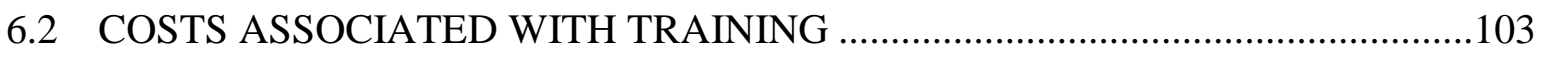

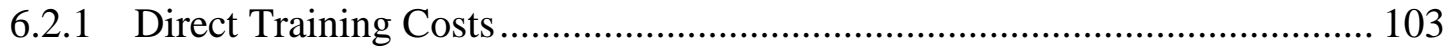

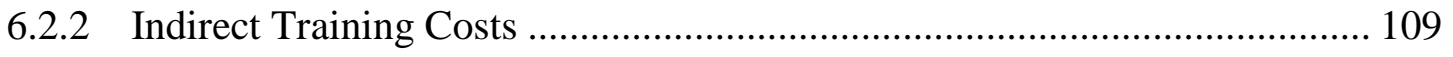

6.3 TOTAL COST FOR DIFFERENT TRAINING GROUPS .....................................110

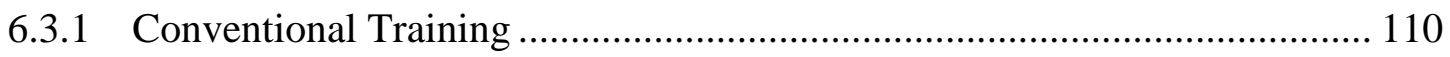

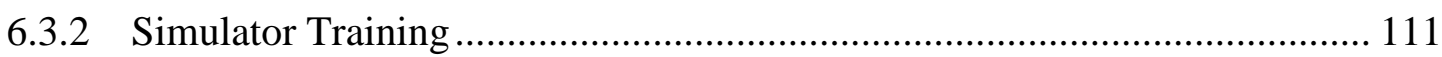

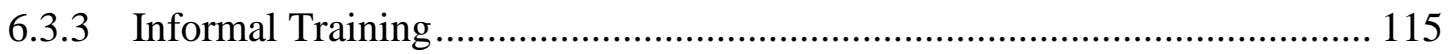

6.3.4 CDL-focused Training ...................................................................... 115

6.4 BENEFITS ASSOCIATED WITH TRAINING .............................................117

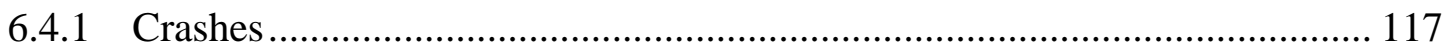




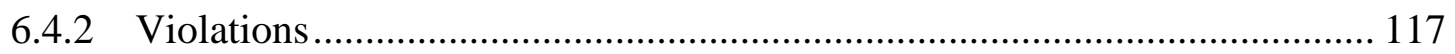

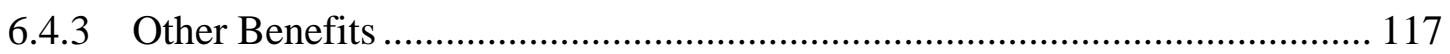

6.5 COST-EFFECTIVENESS ANALYSIS ……………………………………......118

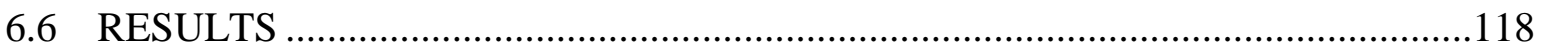

6.6.1 Cost of Simulator versus Conventional Training......................................... 118

6.6.2 Cost of Conventional versus CDL-focused and Informal Training ............... 119

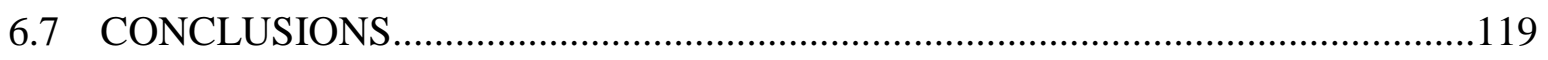

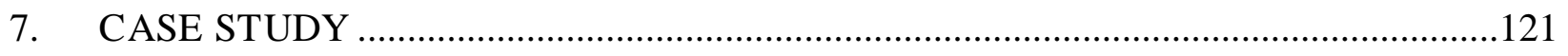

7.1 EXISTING SIMULATOR-BASED TRAINING PROGRAMS ................................121

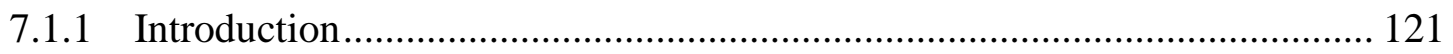

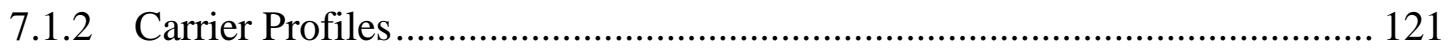

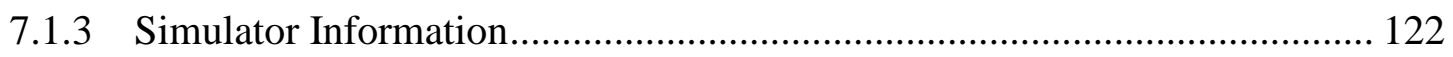

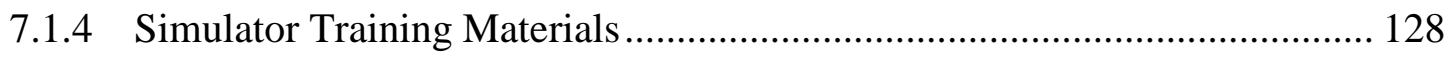

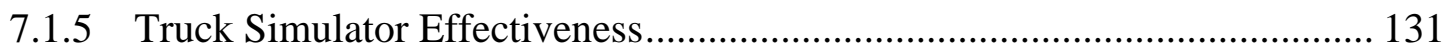

7.2 DRIVERS' PERCEPTIONS OF SIMULATOR-BASED TRAINING ……….........142

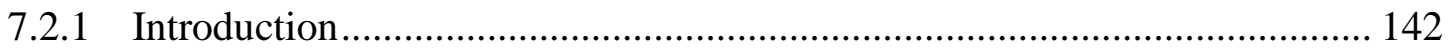

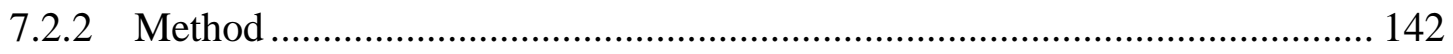

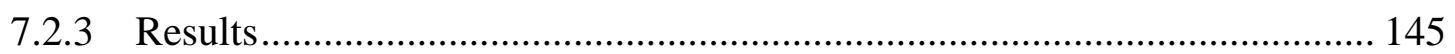

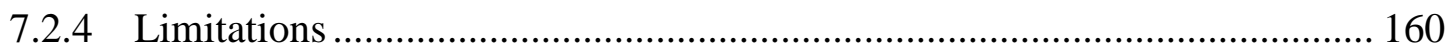

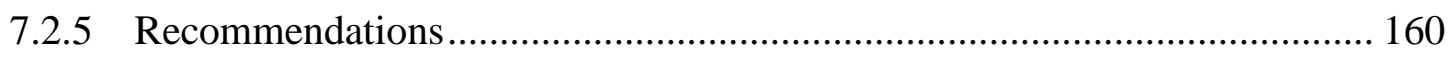

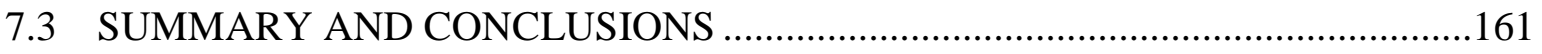

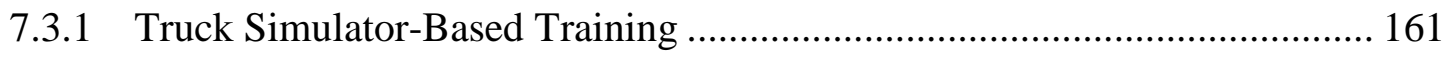

7.3.2 Carrier Implementation of Simulator-Based Training.................................. 161

7.3.3 Driver Opinions on Simulator-Based Training................................................ 162

7.3.4 Future Directions ..................................................................................... 163

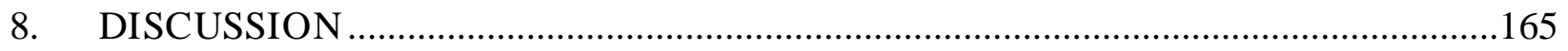

8.1 ENTRY-LEVEL TRAINING STUDY ………………………………………........166

8.1.1 Effects of Training Method on CDL and Related Test Performance ........... 166

8.1.2 Validity of Simulation Training and Testing ................................................ 166

8.1.3 Participant Evaluation of Training after Employment.................................... 169

8.1.4 Effects of Training Method on Driver Performance........................................ 169

8.1.5 Effects of Training Method on Safety Performance Based on Driving Records 169

8.1.6 Effects of Training Method on Employee’s Supervisor Evaluation.............. 170

8.1.7 Exploratory Analysis of "Big Five" Personality Correlates .......................... 170

8.2 ADVANCED CAPABILITIES SHOWCASE .........................................................170 
8.2.1 Assessing Advanced Capabilities of the Simulator .................................... 170

8.2.2 Participants' Assessment of the Simulator's Advanced Capabilities ............ 171

8.3 COST EFFECTIVENESS ANALYSIS OF ENTRY-LEVEL CMV DRIVER

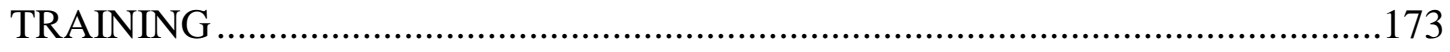

8.3.1 Cost of Simulator versus Conventional Training........................................ 173

8.3.2 Cost of Conventional versus CDL-focused and Informal Training.............. 173

8.4 CASE STUDY ON EXISTING SIMULATOR-BASED TRAINING PROGRAMS174

8.4.1 Carrier Implementation of Simulator-Based Training................................ 174

8.4.2 Driver Opinions on Simulator-Based Training........................................... 175

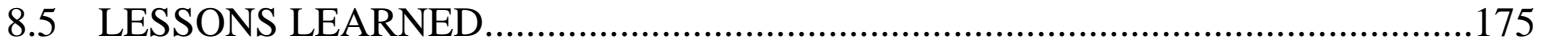

8.5.1 Participant Payments for Travel Time ..................................................... 175

8.5.2 Participant Contact............................................................................. 176

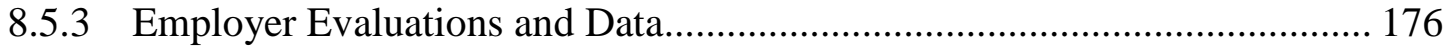

8.5.4 Department of Motor Vehicles Data....................................................... 176

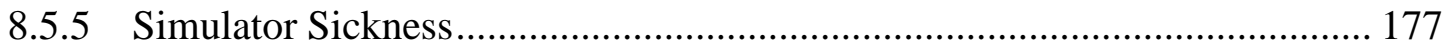

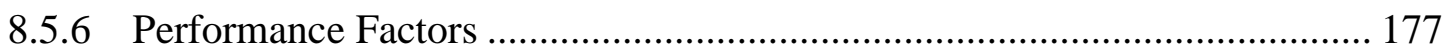

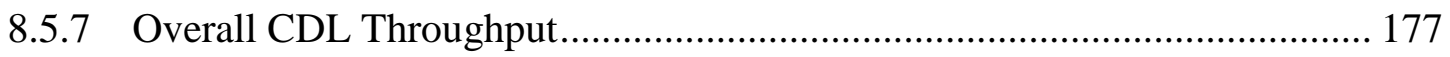

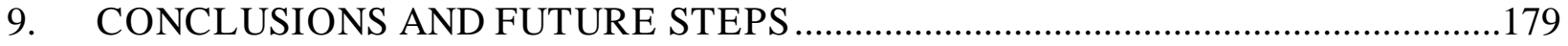

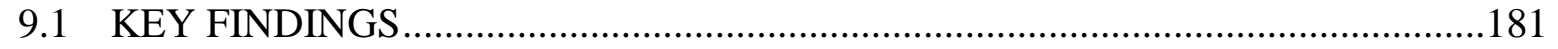

9.2 RECOMMENDATIONS FOR IMPLEMENTING SIMULATOR-BASED TRAINING BASED ON CASE STUDY FINDINGS .........................................182

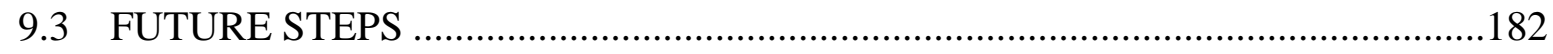

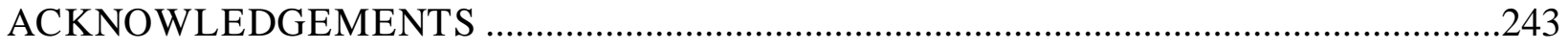

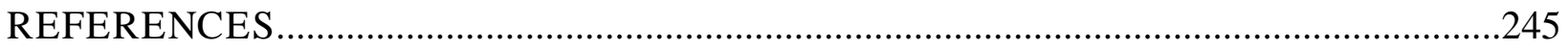




\section{LIST OF APPENDICES}

APPENDIX A—PARTICIPANT SCREENING PROTOCOLS ……………………...........185

APPENDIX B—DTCC ENTRY-LEVEL TRAINING CURRICULUM …………………....193

APPENDIX C—SIMULATOR ORIENTATION PROTOCOL ……………………...............194

APPENDIX D—SIMULATOR SICKNESS SCREENING QUESTIONNAIRE................201

APPENDIX E-ADVANCED CAPABILITIES EMERGENCY MANEUVERS AND

EXTREME CONDITIONS OPERATIONAL DEFINITIONS ……………………......202

APPENDIX F-DELAWARE DMV ROAD TEST, RANGE TEST, AND RELATED

MATERIALS

APPENDIX G-COMMERCIAL DRIVER 4-MONTH FOLLOW-UP SELF-

ASSESSMENT

APPENDIX H-COMMERCIAL DRIVER ON-THE-JOB SAFETY PERFORMANCE

EMPLOYER QUESTIONNAIRE

APPENDIX I-ADVANCED CAPABILITIES TRAINING AND DRIVING HISTORY

QUESTIONNAIRE

APPENDIX J_ADVANCED CAPABILITIES POST-DRIVE QUESTIONNAIRE..........217

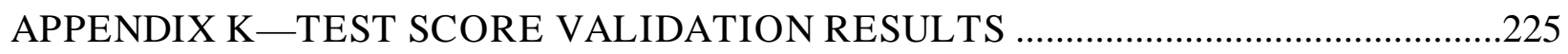

APPENDIX L_END-OF-TESTING PARTICIPANT ASSESSMENT …….......................231

APPENDIX M-SIMULATOR CONFIGURATIONS CURRENTLY IN USE ..................237

APPENDIX N—CASE STUDY FOCUS GROUP MEETING PROTOCOL .......................239 


\section{LIST OF FIGURES}

Figure 1. Photo. Doron Precision 400 CAR simulator (Level 1) ...............................................8

Figure 2. Photo. FAAC TT-2000-V Driver Training Simulator (Level 3) ..................................9

Figure 3. Diagram. Levels of performance control (adapted from Reason, 2008)......................10

Figure 4. Diagram. DTCC CTS BTW curriculum schedule....................................................22

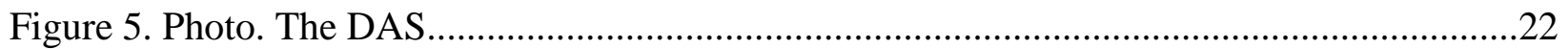

Figure 6. Photo. The TT-2000-V7 Driving Simulator ...........................................................23

Figure 7. Photo. The Rear Visual Channel as Viewed Through the Simulator Mirrors................24

Figure 8. Graph. Attempts taken to pass DMV range test, by group.........................................32

Figure 9. Chart. DMV road and range scores, by group......................................................32

Figure 10. Chart. Original and adjusted mean DMV range scores, by group..............................34

Figure 11. Chart. Mean DTCC and Simulator road test scores, by group.................................35

Figure 12. Chart. Mean DTCC and simulator range test scores, by group.................................36

Figure 13. Chart. Lane departures by group membership and test modality.............................53

Figure 14. Chart. Overall ranking scores for simulator aspects.............................................58

Figure 15. Chart. Participants' recommendations of training type.........................................63

Figure 16. Chart. Road test: Entry level and follow-up scores by test mode and group..............66

Figure 17. Chart. Range test: Entry level and follow-up scores by test mode and group. ............67

Figure 18. Chart. Supervisor ratings of participant control and safety skills. ............................73

Figure 19. Chart. Personality factors, by groups. ................................................................75

Figure 20. Chart. Overall participant responses to emergency maneuvers...................................81

Figure 21. Chart. Overall participant responses to extreme conditions.......................................82

Figure 22. Chart. Doubles participants' ranking of most effective simulator aspects. .................87

Figure 23. Chart. Doubles participants' ranking of least effective simulator aspects...................88

Figure 24. Chart. Tanker trailer participants' ranking of most effective simulator aspects...........90

Figure 25. Chart. Tanker trailer participants' ranking of least effective simulator aspects. ..........91

Figure 26. Chart. Van trailer participants' ranking of most effective simulator aspects...............92

Figure 27. Chart. Van trailer participants' ranking of least effective simulator aspects. ..............93

Figure 28. Chart. Doubles participants' emergency maneuver ratings.......................................95

Figure 29. Chart. Tanker participants' emergency maneuver ratings.......................................96

Figure 30. Chart. Van trailer participants' emergency maneuver ratings...................................97

Figure 31. Chart. Doubles participants' extreme situation ratings. ..........................................98

Figure 32. Chart. Tanker participants’ extreme situation ratings. ...........................................99

Figure 33. Chart. Van trailer participants' extreme situation ratings. .......................................100

Figure 34. Photo. Glass-panel dash and touch screen control panel used at Carrier A. .............122

Figure 35. Photo. Simulator screens, dash, and seat used at Carrier A. ..................................123

Figure 36. Photo. Simulator shift tower used at Carrier A. ..................................................124

Figure 37. Photo. Simulator and playback monitor used at Carrier A......................................125

Figure 38. Photo. Simulator seat, dash, and screens used at Carrier B...................................126

Figure 39. Photo. Simulator OPCON used at Carrier B. .....................................................127 
Figure 40. Photo. Operator's console and simulator used at Carrier B.

Figure 41. Illustration. Driver's view of introductory backing scenario. .130

Figure 42. Illustration. Operator's console overhead view of introductory backing scenario.....131

Figure 43. Graph. Frequency of crash events per driver by training type. .136

Figure 44. Graph. Frequency of participant responses to "Who would benefit most from a truck simulator training program?” .145

Figure 45. Graph. Comparison of participant responses noted as both a similarity and difference in simulator and BTW driving. .148

Figure 46. Chart. Percentage of participants' responses to "What is the ideal time for truck simulator-based training?”

Figure 47. Graph. Participant ratings regarding the effectiveness (or ineffectiveness) of their truck simulator training program.....

Figure 48. Graph. Participant responses to "Describe the principal areas in your simulator program you would retain”.

Figure 49. Graph. Participant responses to "Describe the principal areas in your simulator program you would modify"

Figure 50. Photo. Delaware DMV CDL exam score sheet (first page).

Figure 51. Photo. Delaware DMV CDL exam score sheet (second page).

Figure 52. Chart. Demerits assigned to the Simulator Training Group, by rater.

\section{LIST OF TABLES}

Table 1. Main Study Findings. xxi

Table 2. Participant Demographics for Entry-level Training Groups..........................................18

Table 3. Participant Demographics across each Trailer Type in the ACS.................................20

Table 4. Operational Definitions of Ranking Aspects .........................................................28

Table 5. Simple Effects Tests for DMV Range Tests.............................................................33

Table 6. Follow-Up ANOVA results for DTCC and Simulator Road and Range Tests ...............35

Table 7. Descriptive Statistics for Each Group's Test Scores...................................................39

Table 8. Simple Effects Tests for Simulator Road and Range Tests ........................................40

Table 9. Number of Participants that Failed each Test............................................................41

Table 10. Correlations among Conventional Training Group Driver Test Scores .......................42

Table 11. Correlations among Simulator Training Group Driver Test Scores ............................43

Table 12. Correlations among Informal Group Driver Test Scores..........................................43

Table 13. Correlations among CDL-focused Group Driver Test Scores ....................................44

Table 14. Comparison of DTCC and Simulator road and rage test scores .................................44

Table 15. Road Test: Descriptive Statistics and Correlations for Scored Aspects of DTCC and

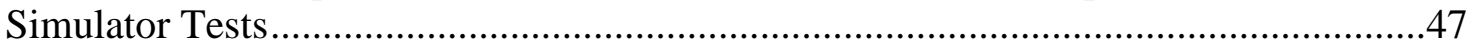

Table 16. Event Rates by Group Membership and Test Modality ............................................51

Table 17. Lane Deviations, by Group Membership and Test Mode...........................................52 
Table 18. Range Test: Descriptive Statistics and Correlations for Scored Aspects of DTCC and Simulator Tests

Table 19. Descriptive Statistics for Participants' Current Self-rating of Control and Safety Skills60

Table 20. Descriptive Statistics for Participants' Retrospective Rating of Training .60

Table 21. Rating of Training at End of Training and 4-months After Being Hired .61

Table 22. Change Scores for Rating of Training.... .62

Table 23. Group Means for Participant Rating of Tests as a Valid and Fair Assessment..... 62

Table 24. Status of Participants for Follow-up Testing. .65

Table 25. Descriptive Statistics for Follow-up Testing . .65

Table 26. Entry-level and 4-month Follow-up Testing Shifting Errors (all 4-month participants)68

Table 27. Entry-level and 4-month Follow-up Testing Shifting Errors. .68

Table 28. Participant Violation Status by Group.... .69

Table 29. Violations Recorded for All Follow-up Participants ....................................................70

Table 30. Crashes Reported to Research Personnel...................................................................70

Table 31. Supervisor Evaluations Availability, by Group ......................................................72

Table 32. Frequency of Supervisor-reported Collisions and Violations, by Group .....................72

Table 33. Correlations and Internal Consistency for Big Five factors ......................................74

Table 34. ANOVA Results for Personality Differences based on Group Membership ................75

Table 35. Regression Model Results for Big Five Factors on Test Outcomes .............................76

Table 36. Training Group Membership Parameter Estimates for each Regression.....................77

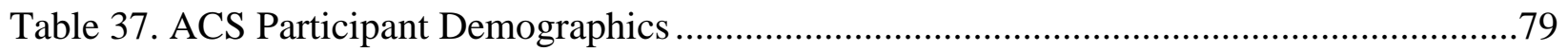

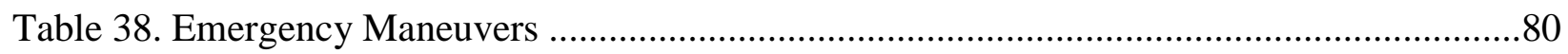

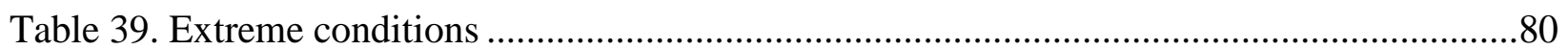

Table 40. Number of Trainee Hours in Each Training Program .............................................104

Table 41. Number of Trainer Hours in Each Training Program...............................................105

Table 42. Facilities and Equipment Costs...........................................................................106

Table 43. Operational Costs Considered to Compute the Average Cost per Mile for Trucks ....107

Table 44. Costs Associated with Truck Use .............................................................................108

Table 45. Costs Associated with Simulator Use ....................................................................109

Table 46. CDL Test Pass Rate for Study Drivers and Cost ...................................................110

Table 47. Study Driver Employment Status and Cost ........................................................110

Table 48. Total Direct Cost of Conventional Group Training ................................................111

Table 49. Total Costs for Conventional Training .................................................................111

Table 50. Total Direct Cost of Simulator Group Training Based on Cost of the Study Simulator112

Table 51. Total Direct Cost of Simulator Group Training Based on Cost of a Mid-Level

Configuration of the Study Simulator

Table 52. Total Direct Cost of Simulator Group Training Based on Cost of a L3 TransSim VS IV114

Table 53. Total Costs for Simulator Training .......................................................................115

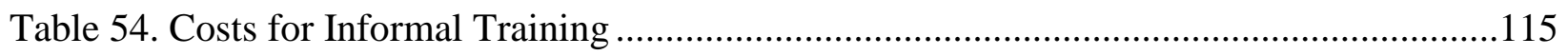

Table 55. CDL-focused Training Program Information ......................................................116

Table 56. Tuition Costs, Trainees’ Time, and Other Costs for CDL-focused Training ..............116 


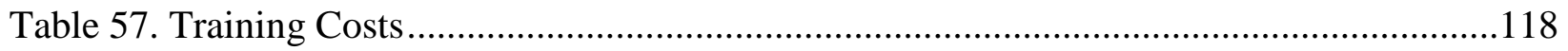

Table 58. Number of Drivers in the Analysis by Training Type and Driving Route ...................132

Table 59. Descriptive Statistics for Driver Tenure ………......................................................133

Table 60. Survival/Failure Analysis for Driver Tenure ..............................................................134

Table 61. Descriptive Statistics for Miles Logged During Tenure .................................................134

Table 62. Survival/Failure Analysis for Miles Logged During Tenure..........................................135

Table 63. Crash Rate per Thousand Miles by Training Type.........................................................137

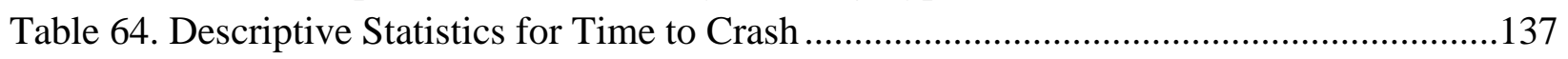

Table 65. Survival/Failure Analysis for Time to Crash by Training Type...................................138

Table 66. Descriptive Statistics for Cost of Crash..............................................................138

Table 67. Descriptive Statistics for Cost of Crashes per Thousand Miles Driven .......................139

Table 68. Major Findings from Carrier A Effectiveness Data.....................................................141

Table 69. Participant Demographic Information ................................................................143

Table 70. Frequency of Participant Responses Regarding Similarities and Differences in Simulator and BTW Driving.

Table 71. Frequency and Percentage of Participants' Response to "Does use of a Truck Simulator Change the Driver Training Process as Compared to Traditional BTW Training?”.

Table 72. Participant Comments Regarding the Ideal Use of Truck Simulators in Entry-level CMV Driver Training

Table 73. Frequency and Percentage of Participants' Responses to "Does the Information you Learn in the Truck Simulator Carry Over to Real-World Driving?”.

Table 74. Participant Responses and Comments Regarding Truck Simulator Transfer of Training155

Table 75. Georgetown DMV CDL throughput from July 2008 to March 2009...........................178

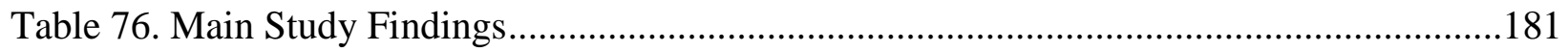

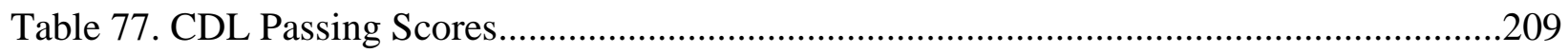

Table 78. Descriptive Statistics for Road Test Demerit Points, by Rater, Group and Test .........226

Table 79. DTCC and Reviewer Discrepancies in Scoring.........................................................228

Table 80. Descriptive Statistics for Range Test Demerit Points, by Rater, Group and Test .......229

Table 81. Simulators Identified as In-Use within the Industry ...................................................237

Table 82. Basic Simulator Specifications by Simulator Model ..................................................238 


\section{LIST OF ACRONYMS}

AAMVA American Association of Motor Vehicle Administrators

ACD AAMVA Code Dictionary

ACS Advanced Capabilities Showcase

AFT-IFTIM Association for the Development of Professional Training in TransportInstitute of Training and Warehousing Techniques

ANOVA analysis of variance

BCA benefit/cost analysis

BTW behind the wheel

CDL commercial driver's license

CDLIS Commercial Driver's License Information System

CMV commercial motor vehicle

CTP Commercial Transportation Program

DAS Data Acquisition System

DMV Division of Motor Vehicles

DTCC Delaware Technical and Community College

FMCSA $\quad$ Federal Motor Carrier Safety Administration

LA longitudinal acceleration

LD lane departure

LTCCS Large Truck Crash Causation Study

MANOVA multivariate analysis of variance

MHQ Motion History Questionnaire

NHTSA National Highway Traffic Safety Administration

NPV net present value

OPCON operator's console 


$\begin{array}{ll}\text { OTR } & \text { over-the-road } \\ \text { PTDI } & \text { Professional Truck Driver Institute } \\ \text { PTT } & \text { partial task trainer/training } \\ \text { SimVal } & \text { CMV driving simulator validation study } \\ \text { SRK } & \text { Skills, rules, knowledge } \\ \text { TTC } & \text { time to collision } \\ \text { USDOT } & \text { U.S. Department of Transportation }\end{array}$




\section{EXECUTIVE SUMMARY}

\section{PURPOSE AND STUDY METHOD}

The Federal Motor Carrier Safety Administration (FMCSA) is dedicated to reducing the incidence and severity of commercial motor vehicle (CMV) crashes. In support of this mission, the research team was commissioned to examine the use and effectiveness of driving simulation as a training and testing tool for entry-level CMV drivers, as well as to examine the advanced capabilities of the driving simulator to replicate emergency maneuvers and extreme situations. Prior findings within this series of research are summarized in Carroll and Dueker (1996), Emery, Robin, Knipling, Finn, and Fleger (1999), and Pierowicz et al., (2002); these findings were used for the basis of the current study. The present study examines the effectiveness of CMV driving simulators for purposes of commercial (large combination-unit truck/tractortrailer) entry-level driver training and State Division of Motor Vehicles (DMV) driver testing. The study includes a general assessment of the effects of different lengths, forms, and venues of CMV driver training on skill acquisition and on-the-job performance, as well as an examination of the cost of these training types. Data collection began August 2006 with training and performance data from 107 entry-level CMV drivers as they studied for and acquired their Class A commercial driver's licenses (CDL) and began driving in a professional capacity. Data were collected during their instruction (for those receiving formal training) on both a simulator and real trucks, and included instructor-scored exams as well as video and kinematic data. Four different groups of drivers, each with different types of training, were examined in this process:

The Conventional Training Group experienced a Professional Truck Driver Institute (PTDI) certified 8-week Class A CDL preparatory course at the Delaware Technical and Community College (DTCC). There were 33 participants in the conventional training group.

The Simulator Training Group experienced the same classroom curriculum as the conventional training group. However, instead of receiving 100 percent of their driving training in a real tractor-trailer, they received approximately 60 percent of their road and range training in a CMV driving simulator. There were 32 participants in the simulator training group.

The Informal Training Group received no formal CMV driver training. These participants reported receiving training from friends or family members, and did not receive structured road and range driving practice. There were nine participants in the informal training group.

The CDL-focused Training Group consisted of participants who graduated from short duration courses (typically less than 4 weeks in duration) that provided training aimed at enabling the participant to pass the State's Class A CDL test. There were 33 participants in the CDL-focused training group.

In addition, this study examined a simulator's advanced capabilities to test driving skills under extreme conditions and emergency maneuvers, with different vehicle cargo/load configurations (e.g., van, double, and tanker trailers). A separate group of 48 Class A CDL drivers with 
differing levels of experience served as participants in this part of the study, termed the Advanced Capabilities Showcase (ACS) sub-study. Each driver had experience with a different cargo/load configuration and provided feedback as to the simulator's performance in the emergency maneuvers and extreme events encountered. Finally, the study includes a case study of existing simulator-based CMV driver training programs. This consisted of an examination of two large motor carriers' implementations of a simulator training program. In addition to site visits and discussions with training personnel from each carrier, focus groups were conducted with drivers who had recently completed each carrier’s training program.

Overall, this study examined five major areas:

The effects of training method for the CDL and related test performance.

The validity of simulation training and testing.

Longitudinal effects of training method.

Exploratory analysis of the Big Five personality correlates.

Assessment and ratings of the advanced capabilities of the simulator.

The results of these topic areas, and the findings from the cost-effectiveness analysis and case study of existing simulator-based training programs, are summarized herein.

\section{EFFECTS OF TRAINING METHOD FOR THE CDL AND RELATED TEST PERFORMANCE}

This research addressed the effect of training methods in a skills measure of driver performance: specifically, whether or not the training method affected road and range test performance. Three different administrations of the road and range tests were examined. One was administered by the State DMV (termed DMV tests), the second was administered by DTCC personnel in real trucks (termed DTCC tests), and the third was administered by DTCC personnel in the simulator (termed Simulator tests). All tests were conducted to the same standards and were scored with the same criteria. Results indicated no difference between training types on DMV road test scores. There was a difference in DMV range test scores, suggesting that conventional group participants scored higher than CDL-focused or informal group participants; however, this statistical difference was not deemed to constitute a meaningful effect, as all groups had mean scores over 90 percent on DMV range tests.

Performance on DTCC and Simulator versions of the DMV road and range tests was examined. These tests used the same scoring criteria and administration as the DMV road and range tests. Additionally, prior to analysis of the DTCC and Simulator road and range tests, independent reviews of a sample of these scores were performed and indicated no scoring bias. Significant training group differences were present. The conventional and simulator groups both scored significantly higher than CDL-focused or informal groups on the DTCC and Simulator road tests. In DTCC range tests, the conventional, simulator, and informal groups all scored higher than the CDL-focused group. In the Simulator range test, the simulator group scored higher than any of the other three groups. These findings suggest that there may be significant skill differences between groups. 


\section{VALIDITY OF SIMULATION TRAINING AND TESTING}

The performance of a simulator (FAAC Model TT-2000-V7 3 DOF) for CMV driver training and testing was investigated by examining the specific scored aspects of the road and range tests. Only moderate to low correlations between simulator test and behind-the-wheel (BTW) test (performed in a real vehicle) were observed. The only training group that had a significant correlation between the Simulator tests and DMV test counterparts was the simulator training group. Additionally, a large number of participants failed the simulator road test (at least one participant from each group, including the Simulator group, and all nine Informal group participants). Only participants from the conventional and CDL-focused training groups had a significant, albeit moderate, correlation between the Simulator and DTCC road tests.

In order to better examine the use of the simulator for CMV driver training and testing, comparisons were performed examining the kinematic data collected during DTCC and Simulator testing. The rates of lane departure (LD), time-to-collision (TTC), and longitudinal acceleration (LA) events were compared. Simulator and conventional groups were shown to have similar LD rates in DTCC testing, indicating that the participants who received most of their training in the simulator had vehicle lateral control skills equivalent to those of conventionally trained participants. This suggests that the simulator has validity for training entry-level CMV drivers for road driving. Similarly, when those participants who experienced PTDI-certified training (the conventional and simulator groups) were compared against the participants who did not have PTDI-certified training, a difference in LD rate was observed during DTCC testing. In the DTCC road test, drivers with PTDI-certified training had a significantly lower LD rate (i.e., were less likely to have a LD) than those drivers who did not have PTDI-certified training.

\section{LONGITUDINAL EFFECTS OF TRAINING METHOD}

Participants were followed after licensure in order to observe the effects of training method on participants' evaluation of their training method, driving performance after working as a Class A CDL driver, safety performance, and supervisory ratings. This effort was complicated due to an economic downturn occurring during the course of data collection. Thus, many participants were not able to find a job as a Class A CDL-licensed CMV driver. This resulted in a small sample for the longitudinal follow-up studies. Only 22 participants were able to complete the follow-up evaluations after 4 months on the job.

However, findings for participant evaluation of training appear to be consistent across all four training groups. All participants thought they were better than average in terms of control and safety skills and, on average, felt their training method was average or above average. When they were evaluated in a simulated environment, most participants thought that the simulator assessment was fair. All of the participants would recommend the training method they selected to obtain their entry-level training, with the exception of one informal group participant.

Most of the follow-up DTCC and road and range test scores were lower than the original scores for the entry-level tests. However, conventional and simulator group participants performed better on the follow-up road tests than did participants from the informal and CDL-focused 
groups. Possible explanations for these results are found in the fact that some participants used a different type of transmission in their work vehicle, did not regularly practice the tested backing maneuvers, or had different driving exposure (i.e., local delivery versus over the road).

Driving records for participants in the 4-month post-training follow-up were examined. Because of the small sample size, short time frame, and the low frequency of violations, too few records were present for statistical analysis. However, it did appear that there were proportionally equivalent numbers of violations across groups.

Supervisor ratings were obtained for participants in the 4-month post-training follow-up. In addition to the low sample size, only 10 participants' supervisors agreed to participate. For the data that were collected, there appear to be no major differences in supervisory ratings across groups; all training groups received a control and safety skills rating of above average with a tendency towards the side of best performance.

\section{EXPLORATORY ANALYSIS OF THE BIG FIVE PERSONALITY CORRELATES}

An exploratory analysis of the relationship between personality traits and training outcomes was conducted. Each participant in the entry-level study completed a personality inventory assessing Big Five personality factors (openness, conscientiousness, extraversion, agreeableness, and neuroticism; Goldberg, 1992). These five factors are well-established in research, represent the broad dimensions of human personality, and were included to examine what affect (if any) personality had on training. Analyses revealed no significant group differences in any of the Big Five personality factors. Likewise, no personality factor was found to significantly predict training outcomes on the DMV, DTCC, and Simulator versions of road and range tests. Therefore, it is suggested that skill test scores are not predicted by personality factors.

\section{ADVANCED CAPABILITIES OF THE SIMULATOR}

Following a 1-hour introductory exposure to the simulator, 48 experienced CMV drivers completed a series of maneuvers in a simulator that included 12 emergency maneuvers in order to examine the ability of the simulator to replicate these advanced driving scenarios. The 12 emergency maneuvers were:

Merge squeezes.

Lane crosses.

Steering tire blowout.

Rollovers (both to the right and left).

Brake failures.

Evasive maneuvers.

Animal crossings.

Blind entrances. 
Pedestrians.

Tight city turns.

Roadway obstructions.

And 10 extreme conditions were tested:

Fog.

Rain.

Snow.

Black ice.

Eight percent upgrades

Eight percent downgrades (dry).

Eight percent downgrades (snow).

Dirt roads.

Construction zones.

Railroad crossings.

Participants were rated on their performance in each scenario and provided subjective ratings for each scenario.

Both million-milers (those drivers with at least 1 million miles of commercial driving logged without a safety incident) and non-million milers (those drivers with less than 1 million miles of commercial driving logged without a safety incident or drivers who have had an at-fault safety incident) tended to respond appropriately for the emergency maneuvers and extreme condition scenarios. In both cases, million milers tended to respond more appropriately than non-million milers. Likewise, in both emergency maneuvers and extreme conditions, a limited number of conditions produced the majority of incorrect responses. For the emergency maneuvers, these scenarios were brake failure, front steering tire blowout, pedestrian encounter, merge squeeze, and evasive action. For the extreme conditions, these scenarios were the railroad crossings, black ice, 8-percent upgrades, and 8-percent downgrades.

Most drivers provided favorable ratings for the ability of the simulator to replicate the emergency maneuver and extreme conditions, and there were few differences in ratings between million milers and non-million milers.

These findings suggest that the advanced capabilities of the simulator hold potential for training experienced drivers for rare or otherwise hazardous situations, allowing drivers to encounter extreme events and undertake emergency maneuvers in a safe, controllable, and replicable manner. This indicates that the CMV simulator may be used for experienced driver refresher and defensive driving training with these types of scenarios. 


\section{COST-EFFECTIVENESS ANALYSIS OF SIMULATOR-BASED TRAINING}

A cost-effectiveness analysis of the four types of entry-level CMV driver training was conducted. This analysis took into account the costs associated with each type of training, including factors such as labor, facilities, truck costs, etc. Only a small difference in direct cost between the simulator and conventional training methods was identified, with simulator-based training costing \$35 less per student per training period (with the training period assumed to be an entire CDL training course) over conventional training methods. Other, less expensive, simulator configurations that were examined in the cost-effectiveness analysis demonstrated further potential cost savings (the use of these for CMV driver training was not examined in this study).

\section{CASE STUDY OF EXISTING COMMERCIAL DRIVER TRAINING SIMULATION PROGRAMS}

Two carriers’ existing simulator-based CMV driver training programs were examined. Quantitative data provided by one carrier indicated that driver tenure (in terms of duration and miles logged) appeared to be positively affected by the truck simulator-based driver training. Although few drivers had crashes recorded (approximately 6 percent of the total sample), drivers who received simulator training had the lowest overall cost per crash and cost per crash per thousand vehicle miles traveled compared to both the mixed simulator/BTW- and exclusive BTW-trained drivers. Results from focus groups indicated that the majority of participants had positive opinions regarding their carrier's truck simulator training program, and that the method of implementation into their corporate culture was key to its success. Areas for improvement focused on hardware and software modifications, additional training uses, and changing how the training was implemented.

\section{CONCLUSIONS}

The present study examined training methods and CDL test performance, the validity of CMV simulator training and testing, longitudinal effects of different training methods, personality factors and training outcomes, and the advanced capabilities of the simulator for simulating emergency maneuvers and extreme events. In addition, a cost analysis of training and a case study of existing CMV simulator training programs were performed. Main study findings are detailed, by topic area and appropriate research question, in table 1 . While the present study demonstrated the potential for CMV simulation in training entry-level Class A CDL drivers, it also identified some key areas of future research, including:

Is there a need for standardized (BTW and simulator) novice-driver training scenarios and equipment?

What are the "best practices" to ensure the transfer of simulator training to BTW operations?

What are the long-term safety implications of different training methods and modalities?

Is there a benefit to providing standardized training to CDL licensing examiners?

Should simulator training hours be treated as the equivalent of BTW training hours? 
Can experienced CMV drivers benefit from training on simulators with advanced capabilities?

Can the simulator be used to deliver targeted defensive driving training?

Table 1. Main Study Findings

\begin{tabular}{|c|c|}
\hline Topic Area & Major Findings \\
\hline $\begin{array}{l}\text { Training Method and } \\
\text { CDL/Related Test } \\
\text { Performance (Research } \\
\text { Question 1) }\end{array}$ & $\begin{array}{l}\text { - There are no training group differences in DMV road tests, and few } \\
\text { group differences in DMV range tests } \\
\text { - There are differences between groups on DTCC and Simulator } \\
\text { versions of DTCC tests, suggesting that more training leads to better } \\
\text { performance on these tests }\end{array}$ \\
\hline $\begin{array}{l}\text { Validity of Simulation } \\
\text { Training and Testing } \\
\text { (Research Question 2) }\end{array}$ & $\begin{array}{l}\text { - Only moderate to low correlations between Simulator and DTCC tests } \\
\text { are present across all groups } \\
\text { - Simulator-trained participants demonstrated significant, but moderate, } \\
\text { correlations between Simulated and DTCC tests } \\
\text { - The simulator may be used to train many aspects of road and range } \\
\text { driving, however it cannot be used for testing those aspects at the } \\
\text { present time } \\
\text { - There are no differences in real-world vehicle lateral control between } \\
\text { Conventional and Simulator training group participants. This is } \\
\text { evidence that the simulator is a valid method for training entry-level } \\
\text { CMV drivers } \\
\text { - Participants trained in a PTDI-certified program have better lateral } \\
\text { control over a vehicle than participants not trained in a PTDI-certified } \\
\text { manner }\end{array}$ \\
\hline $\begin{array}{l}\text { Longitudinal Effects of } \\
\text { Training Methods (Research } \\
\text { Question } 3,4,5, \& 6)\end{array}$ & $\begin{array}{l}\text { - Most participants had generally high self-ratings of control and safety } \\
\text { skills, and would recommend their manner of training to others } \\
\text { interested in learning to drive a CMV } \\
\text { - Both Simulator and DTCC follow-up road and range test scores were } \\
\text { lower for all groups as compared to entry-level final tests } \\
\text { - No differences in safety records were able to be determined } \\
\text { - No differences in supervisory ratings were found }\end{array}$ \\
\hline $\begin{array}{l}\text { Personality Correlates } \\
\text { (Research Question 7) }\end{array}$ & - Personality does not predict performance on road and range tests \\
\hline $\begin{array}{l}\text { Advanced Simulator } \\
\text { Capabilities (Research } \\
\text { Question } 8 \text { \& 9) }\end{array}$ & $\begin{array}{l}\text { - ACS participants generally rated the scenarios as realistic } \\
\text { - Million milers responded to simulated events appropriately more often } \\
\text { than non-million milers }\end{array}$ \\
\hline $\begin{array}{l}\text { Cost-effectiveness Analysis } \\
\text { of Simulator-based Training }\end{array}$ & $\begin{array}{l}\text { - Simulator training is associated with a small (\$35/participant) cost } \\
\text { savings over Conventional training } \\
\text { - Simulator training costs are likely to decrease over the foreseeable } \\
\text { future due to decreases in the cost of simulators }\end{array}$ \\
\hline $\begin{array}{l}\text { Case Study of Existing CMV } \\
\text { Simulator Training Programs }\end{array}$ & $\begin{array}{l}\text { - Carriers are successfully using CMV simulators to train entry-level } \\
\text { drivers and provide refresher training for existing drivers } \\
\text { - Drivers have positive opinions regarding simulation-based training }\end{array}$ \\
\hline
\end{tabular}


[This page intentionally left blank.]

xxii 


\section{INTRODUCTION}

\subsection{PROJECT OVERVIEW}

During the economic period leading up to the beginning of the current study, commercial motor vehicle (CMV) fleets experienced intense competition to hire and retain qualified drivers. Additionally, the industry had forecasted demographic trends through the next decade that predicted a reduction in the number of available qualified CMV drivers (Howard, Zuckerman, Strah, \& McNally, 2009). The economic conditions within the United States during the past 2 decades, together with the high turnover rate that usually characterizes this industry, created a sharp increase in demand for trained CMV drivers that many carriers were struggling to meet. The industry viewed simulation-based training as a potential way of increasing the number of qualified drivers and, to this effect, interest in CMV driving simulators for both training and testing increased (Dugan, 2008).

This is not an unfounded interest, as a continuing trend in the CMV industry is the increasing sophistication of, as well as reliance upon, training and personnel technologies. These technologies have included such systems as validated selection methods, onboard monitors for performance evaluation, and driving simulators for training. Yet driving simulators hold particular promise for enhancing commercial driver training and testing. However, the implementation of CMV simulators is more difficult than the successful implementation of an automotive driving simulator. This is because tractor-trailer driving is a complex sensory-motor and cognitive task that is, in many ways, dissimilar to car driving. Differences in vehicle size, handling, direct and indirect visibility, and stability make the operation of large articulated vehicles a qualitatively different task compared to driving a passenger vehicle. In addition, the predominant types of crashes and magnitudes of crash consequences are different for heavy vehicles and passenger vehicles, requiring truck operators to receive special training.

Fortunately, many of the unique skill requirements for operating large trucks may be addressed through the use of advanced simulation technology, which is continuously becoming more capable, reliable, and affordable. Over the past decade or more, high-fidelity, "full mission" truck driving simulators have become commercially available at steadily decreasing prices. Hartman et al., (2000) report that many European countries have successfully included simulators in CMV driver training programs. In a public/private partnership, a French training program, the Association for the Development of Professional Training in Transport-Institute of Training and Warehousing Techniques (AFT-IFTIM) offers a curriculum combining simulator- and computer-based training in addition to behind the wheel (BTW) training, with a program to track individuals' training hours. The Stora Holm vocational center in Göteborg, Sweden, uses a similar combination of simulator-, computer-, and BTW-based training for entrylevel CMV drivers. In both the AFT-IFTIM and Stora Holm experience with CMV simulators, results have suggested benefits to simulation-based training. In particular, the AFT-IFTIM program considers 1 hour of simulated driving and 4 hours of BTW driving to be more effective than 8 hours of BTW driving.

Findings such as these have led some training programs and motor carriers within the United States and Canada implementing simulator-based CMV training programs (Robin et al., 2005a). 
However, many questions about how these programs would work in the much different CMV driver training and testing environment of the United States remain. In order to better explore the potential advantages of simulation training, the Federal Motor Carrier Safety Administration (FMCSA) has established and sponsored a CMV driving simulator validation (SimVal) research program. Prior SimVal research efforts built on the previous research in CMV driver simulationbased training (Carroll \& Dueker, 1996; Emery, Robin, Knipling, Finn, \& Fleger, 1999; Pierowicz et al., 2002). The principal purpose of the current study is to examine how simulator technology, compared to conventional training methods, may facilitate and enhance tractortrailer driver training and long-term driving performance and safety, as well as to determine the testing implications for commercial driver's license (CDL) candidates. This study attempts to ascertain the overall safety benefits of training itself by comparing the research results in four training methods:

Conventional Training — defined as conventional, Professional Truck Driver Institute (PTDI)certified, BTW training.

Simulator Training—defined as simulator-based, PTDI-certified, training with 58 percent of driving time spent in a simulator and 42 percent of driving time spent BTW.

Informal Training — defined as unstructured training provided outside of formal training settings, with non-professional trainers (e.g., drivers trained by friends or family members).

CDL-focused Training — defined as short, CDL-exam focused, truck driver training courses.

The current study had three major objectives:

The study sought to determine how these four different entry-level training types compare in terms of skills acquisition and forward transfer of training to on-the-job driver performance.

The study investigated the relationship between type of training method and actual job performance through a longitudinal follow-up of participants in the entry-level study.

The study demonstrated the advanced capabilities of the simulator to determine the appropriateness of simulation for testing tractor-trailer drivers on particular maneuvers (e.g., emergency maneuvers and extreme conditions) and vehicle configurations (e.g., vans, tankers, and doubles trailers). 


\section{BACKGROUND}

\subsection{NEED FOR INCREASED TRAINING}

Dueker (1995) concluded that entry-level CMV drivers were not receiving adequate training prior to beginning their professional driving careers. This finding is further evidenced by the large number of in-house training programs (commonly known as “driver finishing” programs) operated by carriers (Knipling, Hickman, \& Bergoffen, 2003). These programs provide newly licensed CMV drivers additional training and/or supervised driving time to ensure that a minimum operational skill level is met. Stock (2001) indicated that approximately 75 percent of carriers operate driver finishing programs.

In order to legally operate a tractor-trailer, drivers are required to hold a Class A CDL. There is a clear need for a greater capacity of high quality CMV driver training (Dugan, 2008). Although there are Federal standards for testing and issuing Class A CDLs provided in the Commercial Motor Vehicle Safety Act of 1986, there is no current Federal requirement for training CMV drivers. CMV driver trainers have noted the need for more effective training strategies to increase the tenure and skills of new drivers (Dugan, 2008). Some industry groups have created independent training standards for use in CMV driver training (PTDI, 1999). A number of entrylevel, driver finishing and CMV driver training programs currently use PTDI standards. Truck simulators could potentially be a tool to increase the efficiency and quality of novice CMV driver training. Thus, there is interest in examining their use in CMV driver training programs.

\subsection{PRIOR SIMVAL RESEARCH}

A number of earlier SimVal-related research projects sponsored by FMCSA led to the current study. An initial study (Carroll \& Dueker, 1996) reviewed the state-of-the-art in commercial driving simulation and assessed its readiness for use in commercial driver training. Because simulation technology continued to advance in the late 1990s, FMCSA commissioned an update on simulation technologies in order to reassess the state-of-the-art and evaluate the capabilities of various simulators to meet commercial driver training needs (Pierowicz et al., 2002). The effort involved several tasks, including an extensive literature review, the selection of a candidate truck driving training school, selection of an appropriate simulator, and the development and initial testing of training and evaluation units.

The Commercial Transportation Program (CTP) at Delaware Technical and Community College (DTCC) in Georgetown, DE, was selected as the study's truck driver training school based on a number of factors. These included their association with an established public institution, support of the institution leadership and staff, qualified professional instructional staff, certification from PTDI, adequate facilities and resources for the simulator, adequate student throughput, high CDL pass rates, nearby State CDL test sites, and being located in a State using the most recent Division of Motor Vehicles (DMV) CDL test procedures.

To determine the optimal simulator for use in the study, researchers reviewed the capabilities of available commercial driving simulators and elected to use the FAAC Model TT-2000-V7 3 
DOF Simulator. As the development and initial testing of training and evaluation units was completed with this simulator platform, drawing conclusions on the performance of other truck simulators based on these results may not be advised.

\subsubsection{Types of Training}

Four forms of entry-level driver training were identified by the researchers. These included conventional, simulator-based, informal, and CDL-focused training methods. Each are briefly described as below.

Conventional Training-Conventional entry-level training occurs in both classroom and BTW settings. Students receive instruction on concepts, techniques, and fleet safety. In addition, there is time for practicing skills maneuvers, proper shifting, and responding to situations while driving trucks on public roads under the supervision of trainers. In order to obtain PTDI certification, these courses must include a minimum of 104 hours of classroom time and 44 hours of BTW training per student (PTDI, 1999). It should be noted that some carriers offer PTDIcertified conventional training, however no entry-level drivers in the present study were trained in PTDI-certified courses besides those in the conventional and simulator groups.

Simulator-Based Training-The Simulator Training Group experienced the same classroom curriculum as the conventional training group. However, instead of receiving 100 percent of their driving training in a real tractor-trailer, they received approximately 60 percent of their road and range training in a CMV driving simulator. There were 32 participants in the simulator training group.

Informal Training - Informal training occurs when a driver receives training in an informal or unstructured setting. No requirements for classroom or BTW time are present and the trainer may not necessarily be certified. Informal training may occur in situations such as an entry-level driver receiving training from a friend or family member.

CDL-focused Training-CDL-focused training shares some of the features of conventional training (including classroom and BTW training) on a compressed schedule. CDL-focused training typically lasts less than 4 weeks total time and involves drivers learning the basic information needed to obtain a CDL instruction permit (i.e., a learner's permit), followed by BTW training for the specific vehicle skills needed to pass the DMV road and skills tests . In addition, in order to meet demand for qualified drivers, some larger carriers may hire employees and provide entry-level driver training though a CDL-focused type of training course. These programs are not eligible for PTDI certification.

\subsubsection{Simulator Based Training Background}

Simulators offer several distinct advantages, as well as certain disadvantages, when compared to the previous three traditional methods of driver training. Many lessons for training CMV drivers may be drawn from the history and development of the early modern-era flight training simulators. One of the earliest flight simulators, the Link Trainer, was developed by aviation engineer Edwin Link to train novice pilots on Instrument Flight Rules. Development of flight simulation was advanced by the immediate need for qualified pilots during World War II. During 
this period, virtually all U.S. Army Air Force trainees received Instrument Flight instructions in Link Trainers (U.S. Air Force, 2008).

Following World War II, flight simulators continued to be developed largely as a need to train novice pilots in less dangerous and less costly environments with greater efficiency. The earliest flight simulators varied from open-loop simulators which provided a psychomotor tracking task simulating the landing of an airplane, to closed-loop motion platform simulators such as the Link Trainer, which provided a higher degree of ecological validity for visual flight rule training. Both types of simulator had the ability to replicate cognitive and motor tasks necessary for real-world flight scenarios. Simulators were demonstrated to have a positive effect on novice pilot training (Orlansky \& String, 1977). Following the military's use of simulators as a training device, many civilian organizations quickly adopted simulation-based training approaches, especially in the use of driving simulators for training automobile drivers (Wachtel, 1995).

\subsubsection{Benefits and Drawbacks to Driving Simulators}

Driving simulators have a number of benefits that have been identified during their use in research and training. Driving simulators are tools for research and training that provide a realistic, yet non-hazardous, replication of the driving experience. Simulation-based training is one area which promises to assist in training future CMV drivers (Brock, Jacobs, Van Cott, McCauley, \& Norstrom, 2001). Several distinct advantages of simulator-based training are identifiable. In addition, simulators offer the opportunity to obtain high quality measures of driver performance and behavior in situations that would be rare, difficult, or dangerous to replicate in BTW training. Robin et al., (2005b) summarized the potential benefits of simulatorbased driver training as:

- $\quad$ Providing for the safety of the driver and vehicle.

- Driving maneuvers that would be difficult and/or dangerous to reproduce (even on a skid pad) are relatively simple to produce in advanced training simulators.

- Drivers can be introduced to scenarios which are infrequent within the roadway environment or would be dangerous for a novice driver.

- Allowing for a higher level of standardization and repeatability in training curriculums.

Modern truck-driving simulators increase the ability of instructors to develop and maintain training scenarios. This standardization in training scenarios and exposure to events should allow for the measurement of a simulator-based training program's long-term effectiveness. It ensures that different students are exposed to the exact same training exercises and scenarios, unlike BTW training, which is subject to variations in environmental conditions and wear of the truck's equipment. The ability of driving simulators to introduce drivers to hazardous and dangerous roadway situations (including traffic interactions, weather, and other driving environment conditions) in a highly efficient and cost-effective manner has prompted some European nations to investigate simulator-based commercial driver training (Parkes, 2003).

As reported in the Brock et al., (2001) study, the effectiveness and efficiency of simulator-based training are its greatest strengths. The efficiency of training is typically higher with simulator 
approaches as compared to BTW training. One reason for this is student throughput. Most fulltask truck simulators require a relatively low ratio of students per instructor during training; however, simulation also offers the ability to decrease the amount of time between starting each student, compared to BTW training, by reducing the time required to service and prepare real trucks, as well as allowing training to occur in poor weather conditions. Additionally, cost savings may be realized with simulator-based training with less use of real trucks, thereby reducing maintenance and fuel costs.

This increase in efficiency is also evident in situations where one trainer is able to instruct multiple students using simple, limited-scope simulators known as partial task trainers (PTT). These simulators are typically limited in the interaction between the user and the simulated environment; however, PTTs are commonly less expensive to purchase than full-task simulators. Instructors are able to supervise and instruct multiple trainees simultaneously using a PTT in a controlled environment. PTTs have been used with great success in the medical simulation community where they are used to train medical professionals on specific aspects of procedures, such as suturing (Kneebone, Nestel, Vincent, \& Darzi, 2007).

Simulation also serves to better engage, and subsequently increase training effectiveness for, drivers. This is typically referred to as presence, or immersion. Presence is defined as a subjective sense of being in one environment while being physically located in another (Witmer \& Singer, 1998) or that the person using the simulation believes they are actually in the target of the simulated environment (Juang \& Alessi, 2000). Presence within simulations is connected with higher operator performance in the simulated task and with enhanced learning in simulatorbased training (Witmer \& Singer). Presence or immersion is not necessarily associated with the degree of physical realism in the simulation; rather, it is dependent on the responsiveness and emotional connection of the user to the simulated task (Riva \& Gamberlini, 2000). Truck simulators that are able to create a sense of presence and connection for the driver are likely to demonstrate beneficial effects in driver skill acquisition compared to less immersive platforms. Additionally, the availability of performance measurements is typically much greater in truck simulators that commonly output driver performance metrics in their standard configuration than in real trucks that must be outfitted with data recording equipment in order to obtain performance metrics. This setup allows the instructor to have quantifiable measures of driver performance while reducing the demand on the instructor to provide in-process feedback and to observe driver performance.

Simulation has significant cost benefits in terms of reduced wear and maintenance. Simulators allow drivers to make mistakes during the training process (e.g., when learning to shift a double clutching transmission) without damage to property or equipment. Skills can be taught in highly controlled environments that may reduce training time needed for skill proficiency and increase trainee throughput.

In addition to these benefits, simulator-based approaches to driver training are associated with some disadvantages. Not all users are able to comfortably use driving simulators due to the phenomena cluster of symptoms known as "simulator sickness." Simulator sickness symptoms result from improper presentation of the visual and/or kinesthetic cues of a simulation that are attributable to the computer-generated graphics. These symptoms typically manifest as retinal image slip, nausea, and disorientation associated with the improper stimulation of the vestibular 
organs (Pausch, Crea, \& Conway, 1992). The cues producing simulator sickness are typically subtle, almost imperceptible, making them difficult for simulation designers to detect or correct. Although it is difficult to predict exactly which drivers will experience simulator sickness symptoms, some efforts have been made toward prediction of an individual's susceptibility to simulator sickness. Motion History Questionnaire (MHQ; Kennedy, Fowlkes, Berbaum, \& Lilienthal, 1992) seeks to predict simulator sickness from a combination of prior vestibular system discomfort and sickness (such as nausea) associated with different motion types (including boats, planes, amusement rides, and automobiles). Recent work (Golding, 2006) has found that including the frequency of nausea experienced with different types of motion (land-, sea-, and air-based travel, as well as amusement rides) as a child and as an adult may lead to higher predictive power. Additionally, work towards the quantification of simulator sickness symptoms has been performed. The Simulator Sickness Questionnaire (Kennedy et al., 1993) consists of a 26-item symptom checklist and provides a quantification of simulator sickness symptoms of nausea, eye strain, and disorientation, as well as a overall (total) simulator sickness score. The use of pre-screening questionnaires, such as the MHQ, and pre-/post-simulator exposure measures, such as the Simulator Sickness Questionnaire, should allow for the effect of the simulator on users to be quantified and eventually reduced through improved simulator designs.

Economic costs are associated with simulation. Almost every driving simulator in use is computer-based and has maintenance and reliability costs associated with the computer hardware and software. Additional costs are associated with training for instructors, maintenance personnel, and instructional designers. Therefore, simulators have associated costs beyond the initial purchase of the equipment.

Driving simulators are also somewhat dependent on their fidelity to determine what skills can be successfully addressed in driver training (Brock et al., 2001). Partial Task Trainer/Training (PTT) and open-loop simulators have found use in training simple responses such as shifting and braking, but have difficulty in training skills that involve complex decision-making and reaction (e.g., merging into traffic or managing vehicle headway). High fidelity simulators, which are typically more costly, are required to train for these complex skills and behaviors (Pierowicz et al., 2002). Therefore, a tradeoff between training capabilities and simulator costs must be considered prior to purchasing a driving simulator.

\subsubsection{Types of Simulators Currently in Use}

Brock et al., (2001) indicated that the basic definition of simulation-based training was "an instructional method that requires students to interact with specific instructional events based on real-world scenarios" (p. 8). The authors also describe three general "levels" of simulators for training CMV drivers. These include open-loop video (Level 1), low-end simulators (Level 2), and mid-range simulators (Level 3). Although these levels are based on classifications of simulation hardware, they provide the foundation for examining the types of truck simulators currently in use.

Level 1, or open-loop (non-interactive) video, is perhaps the simplest training device and includes a basic set of vehicle controls (steering wheel, throttle/clutch/brake pedals) placed in front of a video screen. Multiple consoles may be placed in front of a single screen, allowing many students to train simultaneously. Figure 1 presents an example of a Level 1 simulator. 


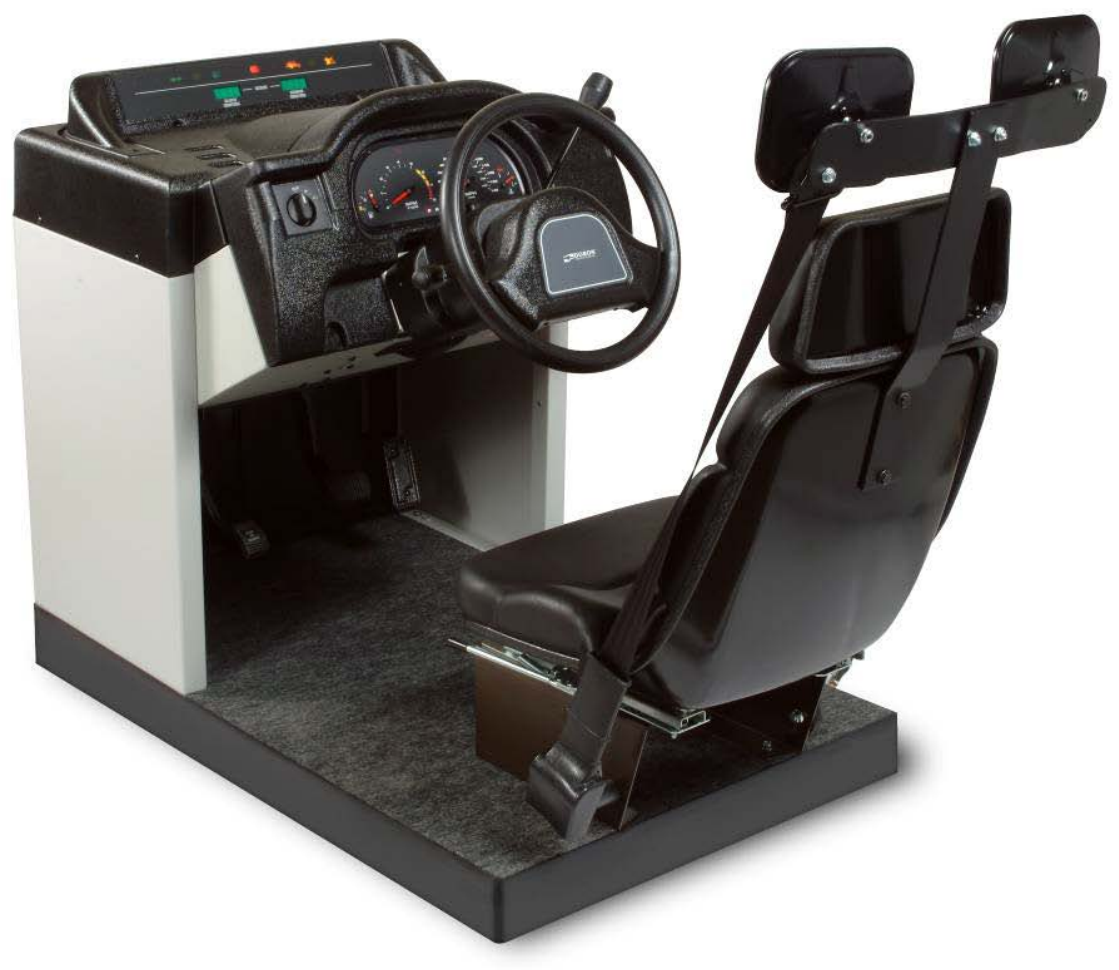

Figure 1. Photo. Doron Precision 400 CAR simulator (Level 1)

Level 1 simulators allow for the training and, depending on the application, recording of specific driving behaviors, such as shifting and braking response. Level 2 simulators are based around gantry-systems and allow the driver to control a scale-model replica vehicle with information presented to the driver by video. Level 2 systems have largely been replaced in the simulation community by Level 3, or mid-range, simulators. Multiple factors that contributed to the decline in popularity of gantry-systems include the advent of lower cost powerful computer graphics, the large amount of space required for gantry boards and arms (used to simulate the driving environment as seen by the simulator driver), the greater flexibility offered by computer-based systems, and the much higher indirect costs of gantry-systems in terms of support personnel. Level 3 simulators are based around a mock-up of an actual heavy-vehicle dashboard (some systems use actual cabins from commercial trucks) and computer-generated graphics and physics models. There is a high level of interaction between driver, vehicle, and environment in Level 3 simulators. In addition, a variety of driving maneuvers and behaviors may be targeted in training. Figure 2 presents an example of a Level 3 simulator. While some commercial off-the-shelf truck simulators based around a personal computer and steering wheel exists, they typically do not have the ability to provide a simulation of important training aspects (such as shifting) or to allow trainers to design complete training scenarios. Therefore, these systems have not been extensively examined in the CMV-simulator research literature. 


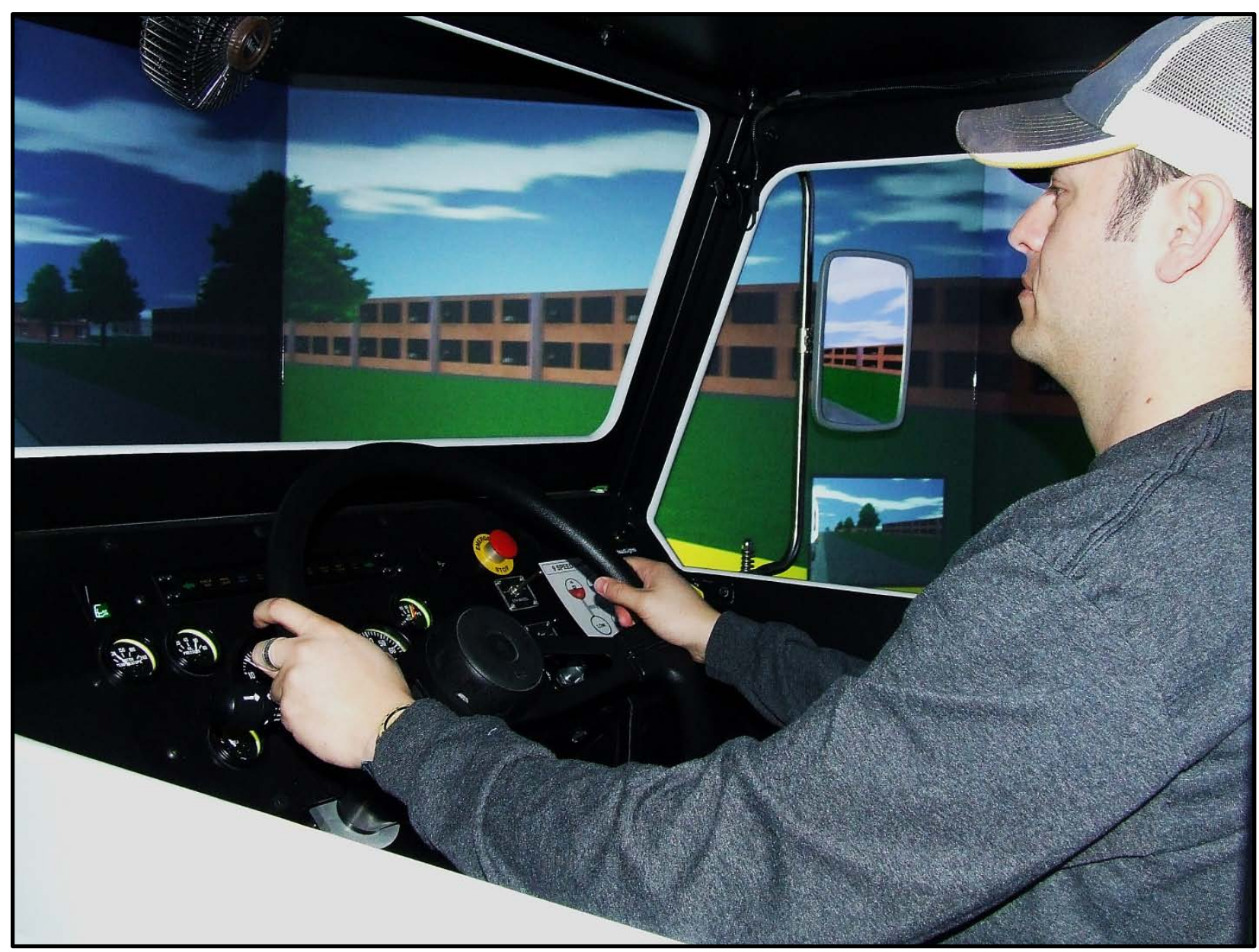

Figure 2. Photo. FAAC TT-2000-V Driver Training Simulator (Level 3)

As with other complex tasks, the operation of a CMV may be viewed through the skills, rules, Knowledge framework (SRK; Rasmussen, 1983). SRK classifies tasks as a function of the cognitive loading and processing required on the individual. Thus, any individual sub-tasks in CMV driving and training may be viewed as a skill-, rule-, or knowledge-based behavior. The lowest cognitive demand behaviors are skill-based, requiring very little of the operator's cognitive resources and operating at an almost automatic level. Skill-based tasks may include a driver's dynamic use of mirrors or an experienced driver's gearshift behavior. As well-learned and practiced behaviors, little conscious control is required to successfully complete these behaviors. Rule-based behaviors require the driver to select and apply an appropriate pre-defined rule(s) to a specific situation. An example of a rule-based behavior is the procedural task of aligning a trailer for docking or responding to a tire blowout. Although more cognitive resources are required to successfully complete a rule-based behavior, the overall cognitive demands presented by the task are typically manageable and do not approach the individual's limits. Knowledge-based behaviors are the most cognitively demanding and include situations where the driver does not have an existing applicable rule. In these situations the driver must complete the task by applying his or her existing knowledge to an unknown situation. Tasks such as "reading the road" or accommodating for improper or misrouted driving directions are knowledge-based tasks. A knowledge-based task requires a high level of cognitive control and 
attention in order to be successfully completed. The range of performance control, based on the level of cognitive processing and difficulty of the situation, is illustrated in Figure 3. Note that as situations transition from routine to more novel scenarios, the level of active cognitive control (or cognitive resources) increases.

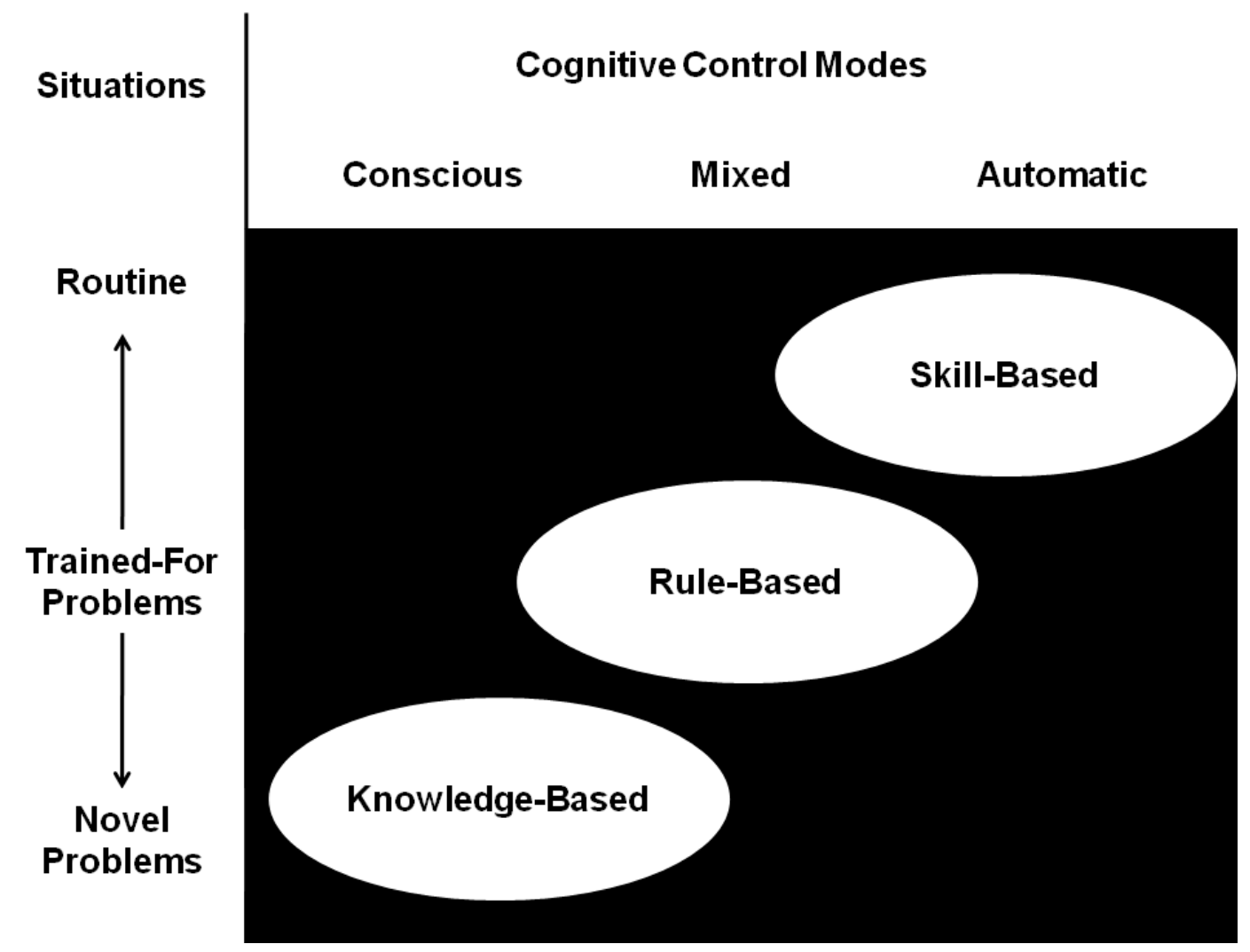

Figure 3. Diagram. Levels of performance control (adapted from Reason, 2008).

The type (i.e., PTT or open-loop) and level of a simulator has a large role in determining the simulator's suitability for training drivers on specific tasks. Although most CMV simulators (both Level 1/PTT and Level 3) accommodate training at the skill- and rule-based level, training that is focused on knowledge-based tasks and behaviors typically requires a more sophisticated simulation facility that specifically accommodates the behavior(s) targeted for training. Additionally, training for higher-level rule- and knowledge-based behaviors may require simulators with a high degree of precision in visual fidelity, motion cueing, and network interaction. This training approach typically costs more than a general use simulator.

The greatly increased availability in commercial-off-the-shelf automobile driving simulators is beginning to be accompanied by greater availability of truck driving simulators. These simulators are typically of Levels 1 and 3 in the Brock et al., (2001) taxonomy. With the more common prevalence of Level 1 and 3 simulators in the training community, it is helpful to examine the practical applications of these simulators.

Level 1 simulators are particularly useful for training basic perception- and response-type tasks, such as the motor coordination required to shift a double-clutching transmission. These simulators provide the trainee with the opportunity to rehearse a skill in a controlled environment 
either independently or under the guidance of a trainer. Level 3 simulators may be used in a similar manner as Level 1; however, their accompanying software provides the ability to disable some functions of the system in order to interact with a limited set of the simulator's features. An example of this is the ability of most Level 3 simulators to select an automatic transmission for drivers focused on the control of an articulated vehicle's position.

Although Level 3 simulators provide this functionality, they can also train for higher level behaviors than Level 1 simulators. These behaviors go beyond basic vehicle maneuvering and control, and include skills such as interactions with traffic and defensive driving. However, the capabilities provided by these machines are typically accompanied by larger space requirements and limitations in the number of trainees who can be processed simultaneously. Whereas openloop and other PTT simulators allow for a large number of students to practice a task simultaneously, Level 3 simulators are designed for a single trainee and trainer to work together. These systems uses a partial-dash mock-up of a truck (some platforms include a truck cabin style enclosure) and at least three channels of image generation to provide visual information to the trainee. All include the option of allowing trainers to select various transmissions, engines, and truck/trailer configurations.

The most common approach to simulator-based training is an integrated program of simulator and BTW training. Drivers in this type of training will receive instruction and practice maneuvers using a driving simulator and later receive coaching during the BTW training. These programs typically use the simulator to either introduce novice drivers to common CMV driving concepts, or to allow drivers to experience a high-risk maneuver in a safe environment. 
[This page intentionally left blank.] 


\section{RESEARCH QUESTIONS}

\subsection{STUDY OBJECTIVES}

The SimVal II study had three major objectives. The first two correspond to entry-level CMV driver training, while the third relates to the advanced capabilities showcase (ACS):

Objective 1: Determine how the four different entry-level training types compare in terms of skills acquisition, as well as in terms of transfer of training between entry-level training and onthe-job driver performance. This study allowed the assessment, in a controlled manner, of the benefits of training itself.

Objective 2: Determine the relationship between type of training method and actual job performance. A longitudinal study was conducted to determine if reliable differences in driver performance based on training type were present. The student drivers' post-training records were examined at 12 months after they obtained jobs where a Class A CDL was required. Additionally, the drivers' performance was measured at DTCC by repeating their BTW and simulator CDL-based skills tests at 4-5 months after they started working in a position where a Class A CDL was required. Measures of driver performance during this part of the study included the frequency of crashes, citations, and supervisory ratings.

Objective 3: Showcase and assess the advanced capabilities of the simulator to determine the appropriateness of simulation to test experienced tractor-trailer drivers on particular maneuvers (e.g., emergency maneuvers and extreme conditions) and vehicle configurations (e.g., van, tankers, and doubles trailers). Moreover, the ACS gathered drivers' opinions on the realism of the simulator's representation of each scenario (e.g., emergency/evasive maneuvers, speed management, extreme driving conditions).

\subsection{RESEARCH QUESTIONS}

Based on the objectives of this research effort, nine key research topics were identified pertaining to the safety benefits of different CMV driver training methods. Each of these topics was explored in research questions.

\section{Topic \#1: Effects of training method on CDL and related test performance}

To evaluate the effects of training methods on driver licensing performance the following research question was examined:

Research Question 1a: Does training method affect DMV road/range test performance?

\section{Topic \#2: Validity of simulation training and testing}

The simulator has the potential to be an effective tool to test drivers' skills on the road and range. To explore this issue, the following research questions were addressed:

Research Question 2a: Can simulation testing adequately assess road/range driving skills? 
Research Question 2b: What is the correlation between scores from the DTCC road/range test and its simulated counterpart?

Research Question 2c: Which test scores (DTCC or simulated) are more predictive of subsequent DMV tests results?

Research Question 2d: What features of the simulator will be the most and least effective for participants primarily trained on the simulator?

\section{Topic \#3: Longitudinal effects of training method on participant evaluation of training}

This research question address participants' perceptions of their own performance at 4-5 months post training as a Class A CDL driver. Additionally, this research question assesses the extent to which participants deem the simulator skill tests as a valid and fair assessment of their driving skills, and if they would recommend their individual training method to other perspective trainees. In order to address this issue the following research questions were examined:

Research Question 3a: Does the training method affect the self-ratings of drivers’ current performance?

Research Question 3b: How does the training method affect participant retrospective self-ratings of the training method received in terms of teaching vehicle control, maneuvering skills, and safe operations in comparison to their peers?

Research Question 3c: Does the training method affect trainee evaluation of training at the end of training and 4 months after being employed?

Research Question 3d: Would participants trained primarily on the simulator deem the simulator skill tests as a valid and fair assessment of their driving skills at the time they completed their training programs?

Research Question 3e: Would participants recommend their method of training to other prospective commercial driver trainees?

\section{Topic \#4: Longitudinal effects of training method on driver performance.}

To study the effects of training method on driver performance the following research question was examined:

Research Question 4a: Does the training method affect performance on the simulated road/range and DTCC road/range test during the retest 4-5 months after the participants start working as a commercial driver where a CDL is required?

Research Question 4b: Does the training method affect the magnitude of change in driving performance $4-5$ months after receiving a CDL?

\section{Topic \#5: Longitudinal effects of training method on safety performance based on driving records}


To explore the training method effect on short term (4-5 months) and long term (12 months), driver performance, on crashes, "points" on the individual's driving record, and citations, the following research question was addressed:

Research Question 5a: Does the training method affect safety performance on-the-job (e.g., incident rates, number of violations, number of crashes, supervisor ratings) after 4 and 12 months on the job?

\section{Topic \#6: Longitudinal effects of training method on supervisor evaluation of employees}

To address the effects of training methods on supervisors' evaluation, the following research question was examined:

Research Question 6a: Does the training method affect supervisors' evaluation of their employees at 4-5 months after being hired and the number of DMV-reported and unreported incidents and violations?

\section{Topic \#7: Exploratory analysis of “Big Five” personality correlates}

To study the correlation between the Big Five personality correlates, the following question was addressed:

Research Question 7a: What is the relationship between the "Big Five” personality correlates (Openness, Conscientiousness, Extraversion, Agreeableness, and Neuroticism) and forward transfer of training and longitudinal effects?

\section{Topic \#8: Assessing advanced capabilities of the simulator}

To assess the advanced capabilities of the simulators the following research question was examined:

Research Question 8a: Are there differences in driver performance between million-miler and non-million-miler drivers for the following types of advanced capability skills in a simulated environment?

Emergency maneuvers by million-miler and non-million-miler drivers corresponding to their experience with different vehicle trailer configurations (e.g., van trailer, tanker trailer, and double-trailer configurations).

Driving under hazardous or extreme road conditions by million-miler and non-million-miler drivers corresponding to their vehicle configuration experience.

\section{Topic \#9: Participants' ratings of the various aspects of the advanced capabilities of the} simulator

To address participants' perceptions of the advanced capabilities of the simulator, the following research questions were examined: 
Research Question 9a: How do both million-miler and non-million-miler drivers rate the usefulness and realism of the simulator for the presentation of the advanced capabilities?

Research Question 9b: Which emergency maneuvers, driving conditions, or other events provided by the simulator are most important and valuable to drivers, and which are rated as the most realistic by the drivers?

In summary, nine research topics were identified as being of significant importance in this project. The research question areas proposed helped guide the data collection and statistical analysis. 


\section{METHOD}

This chapter presents the research method for the two main research efforts under this project:

Entry-level Training Study.

Advanced Capabilities Showcase (ACS).

The details for each of these two efforts are discussed under the following main sections: Participants, Materials, Measures, and Procedure.

\subsection{PARTICIPANTS}

\subsubsection{Entry-Level Training Study}

\subsubsection{Recruitment and Screening}

Participants recruited in the entry-level training study were candidate drivers training for a Class A CDL. Recruitment in the conventional and simulator training groups occurred at the DTCC Commercial Transportation Program (CTP) facility. These potential participants were required to be enrolled in DTCC's CTP as their sole source of Class A CMV driver training. Recruitment took place during the first day of class, which is a lecture-only day.

Participant recruitment in the CDL-focused and informal training groups was conducted on-site by research team personnel during CDL testing at the Delaware DMV offices, under the supervision of and in conjunction with the Delaware DMV CDL Coordinator and the licensing supervisors at each of the four office locations in Georgetown, Dover, New Castle, and Wilmington. Participants were also recruited on a daily basis through newspaper ads and flyers placed in the CDL manuals at the four different DMV locations. Potential participants in the CDL-focused training group were required to have successfully completed a short duration CDLfocused school and have had no other formal training. Potential participants in the informal training group were required to not have prior formal Class A CMV driver training.

The on-site experimenter provided an overview of the study's purpose and objectives to each of the potential participants. A screening questionnaire was then administered to determine eligibility. Prospective participants were required to have the following (see appendix A for the full screening protocol):

- Current Class A CDL learner's permit (conventional and simulator training groups) or current Class A CDL (informal and CDL-focused training groups) obtained within the previous three weeks.

- Have passed a U.S. Department of Transportation (USDOT) physical and drug screening.

- Not have previously held a Class A CDL.

- Females were required to not be pregnant in order to participate. 
As the participant throughput was lower than expected in the informal training group, the research team also recruited from the nearby Maryland DMV locations in the towns of Salisbury and Easton. Recruitment at the Maryland DMV locations was terminated after discovering the Maryland DMV in those localities did not record and save CDL test scores.

\subsubsection{Participants' Demographics}

A total of 124 participants were screened and scheduled to participate in this study; however, nine informal training group participants failed to show for their scheduled meeting to begin participant in the study. Thus, 115 entry-level drivers participated in this study. Five of these participants did not finish the DTCC program and three informal group participants chose to withdraw from the study. A total of 107 participants completed the entry-level testing (see Table 2), including: 33 in the conventional group, 32 in the simulator group, 33 in the CDL-focused group, and 9 in the informal group. All participants in the conventional and simulator groups experienced identical training curricula. While the characteristics of these participants were not experimentally controlled, an effort was made to balance the four groups in regard to age and gender across the study. However, due to constraints of the study design required to execute the present study, there may be other factors not measured by this experimental design that may have influenced either group membership or training/employment outcomes.

Table 2. Participant Demographics for Entry-level Training Groups

\begin{tabular}{|c|c|c|c|c|}
\hline Training Group & $\begin{array}{c}\text { Completed } \\
\text { Entry-level } \\
\text { Training }\end{array}$ & Mean Age & Gender & $\begin{array}{c}\text { Employed Driving a } \\
\text { Truck }\end{array}$ \\
\hline Conventional & 33 & 34 & $\begin{array}{l}31 \text { male, } \\
2 \text { female }\end{array}$ & 10 \\
\hline Simulator & 32 & 35 & $\begin{array}{l}31 \text { male, } \\
1 \text { female }\end{array}$ & 15 \\
\hline Informal & 9 & 40 & $\begin{array}{c}9 \text { male, } \\
\text { o female }\end{array}$ & 9 \\
\hline CDL-focused & 33 & 35 & $\begin{array}{l}30 \text { male, } \\
3 \text { female }\end{array}$ & 10 \\
\hline
\end{tabular}

Due to low throughput at Delaware DMV locations, additional recruitment efforts were undertaken for the informal training group (including advertising and payment for travel time). However, these efforts did not prove successful. An economic downturn resulted in less hiring of entry-level CMV drivers by many carriers, and the total CDL throughput of the Delaware DMV dropped over the study period. It is concluded that the low sample size of the informal group is descriptive of the actual population and not a systematic issue with sampling. This issue is further discussed in section 8.

\subsubsection{Advanced Capabilities Showcase}

The ACS was a demonstration of the potential use of driving simulators' advanced capabilities in driver training and testing. Such advanced capabilities include the ability to simulate emergency maneuvers, extreme road conditions, and different vehicle configurations (e.g., vans, tankers, and doubles). In addition, this showcase explored the realism and effectiveness of each emergency maneuver and extreme road condition encountered while driving the simulator. Drivers with 
differing levels of experience (i.e., million-milers, non-million-milers) were asked to evaluate and provide feedback on the emergency maneuvers and extreme road conditions to determine which emergency maneuvers, driving conditions, or other events provided by the simulator are most important, realistic, and valuable. This participant evaluation could also enhance and provide the basis for improvements in future training of both novice and experienced drivers. The participants recruited for the ACS study were tractor-trailer drivers with a current Class A CDL. Drivers who pull vans, tankers, or a set of doubles were of interest, however, there are no defined guidelines in determining appropriate categorical classifications for drivers' experience levels. Based on discussions with subject matter experts, the groupings for the present study were set at million-miler drivers and non-million-miler drivers. Million-milers were defined as drivers with 1 million miles or more logged and no at-fault crashes. Non-million-miler drivers were defined as drivers who may have completed a driver finishing program and have experience driving the trailer type of interest: van trailers, tanker trailers, and doubles. Non-million-milers are drivers with less than 1 million miles logged or/and any at-fault crashes.

\subsubsection{Recruitment and Screening}

ACS participants were recruited from local trucking companies that had been identified as having certain trailer types (van, tanker, and double-trailer). To facilitate recruitment, posters and flyers were placed at local Delaware trucking companies’ break rooms and other terminal locations. In addition, several trucking companies assisted with driver recruitment.

Once potential participants were identified, the same procedures followed by the on-site experimenter for the entry-level study participants were also followed to determine the eligibility of the ACS participants. However, there were several additional requirements (see appendix A for the ACS participant screening protocol):

- All participants were required to have a current Class A CDL.

- To be driving either a van, tanker, or doubles trailer.

\subsubsection{Participants' Demographics}

A total of 48 participants participated in the study. An additional 12 potential participants who were eligible experienced simulator sickness during the orientation and were unable to participate. Table 3 provides the demographic characteristics across each trailer type for the ACS drivers that participated in the study. 
Table 3. Participant Demographics across each Trailer Type in the ACS

\begin{tabular}{|l|c|l|c|c|c|c|}
\hline Trailer Type & $\begin{array}{c}\text { Number of } \\
\text { Participants }\end{array}$ & \multicolumn{1}{|c|}{$\begin{array}{c}\text { Million-Miler } \\
\text { Status }\end{array}$} & $\begin{array}{c}\text { Mean } \\
\text { Age }\end{array}$ & Gender & $\begin{array}{c}\text { Mean Years } \\
\text { Experience }\end{array}$ & $\begin{array}{c}\text { Vehicle } \\
\text { Miles } \\
\text { Traveled }\end{array}$ \\
\hline Van Trailer & 6 & Million Miler & 51 & $\begin{array}{c}6 \text { male, } \\
0 \text { female }\end{array}$ & 31.3 & $2,942,976$ \\
\hline Van Trailer & 10 & $\begin{array}{l}\text { Non-Million } \\
\text { Miler }\end{array}$ & 44 & $\begin{array}{c}9 \text { male, } \\
1 \text { female }\end{array}$ & 7.7 & 439,100 \\
\hline $\begin{array}{l}\text { Doubles } \\
\text { Trailers }\end{array}$ & 6 & Million Miler & 51 & $\begin{array}{c}6 \text { male, } \\
\text { f female }\end{array}$ & 23.0 & $2,281,976$ \\
\hline $\begin{array}{l}\text { Doubles } \\
\text { Trailers }\end{array}$ & 10 & Non-Million & 42 & $\begin{array}{l}10 \text { male, } \\
0 \text { female }\end{array}$ & 12.0 & 537,687 \\
\hline $\begin{array}{l}\text { Tanker } \\
\text { Trailer }\end{array}$ & 6 & Miler & 51 & $\begin{array}{c}6 \text { male, } \\
0 \text { female }\end{array}$ & 29.5 & $2,538,667$ \\
\hline $\begin{array}{l}\text { Tanker } \\
\text { Trailer }\end{array}$ & 10 & Non-Million & 42 & $\begin{array}{l}10 \text { male, } \\
0 \text { female }\end{array}$ & 10.1 & 402,300 \\
\hline
\end{tabular}

\subsubsection{Participant Protections}

All participants were provided with an informed consent form describing the purpose of the study, procedures, benefits, risks, confidentiality, and participant rights and responsibilities. Participants were provided with time to read the informed consent form and have all questions answered prior to participation. As participation was voluntary, participants were allowed to withdraw from the study at any time.

\subsection{MATERIALS}

\subsubsection{Experimental Venue}

DTCC's CTP collaborated with the research team for this study. DTCC was established in 1966 and the CTP was established in 1971. DTCC has a positive safety record; only three incidents have occurred since 1996. All were property-damage-only incidents (involving no injuries or vehicles being put out-of-service) that occurred on the range in 2005. Following these incidents, a review of instructor guidelines was conducted to help prevent any future incidents. For the duration of data collection efforts at DTCC, the CTP has been certified by PTDI since 2000. PTDI provided a waiver to allow the use of a driving simulator to count towards each student's driving time who participated in this study. Use of the DTCC facility included the use of four of DTCC's Class-8 trucks with trailers as well as the driving range, and collaborations with DTCC instructors.

\subsubsection{Entry-Level Training Curriculum}

\subsubsection{Conventional and Simulator Training Groups}

Participants in the conventional training group received full-curriculum entry-level training. These participants were students at the DTCC CTP and received 50 hours of BTW training in an actual tractor-trailer and 147 hours of classroom instruction during this PTDI-certified program. The entire duration of the training course was 8 weeks, including all classroom instruction 
(which includes instruction on vehicle systems, theory of vehicle operations, log books, and Federal Motor Carrier Safety Regulations), range driving (backing maneuvers), and road driving. Similar to the conventional group, participants in the simulator group were students at DTCC and followed the same full-curriculum entry-level training. However, they received 42 percent (23 hours) of their practice driving time in a real tractor-trailer and 58 percent (32 hours) in a simulator while also receiving the same 147 hours of classroom instruction. The simulator and BTW hours provided above do not include any testing time. A minimum of 44 hours of driving time is needed to receive the PTDI certificate. All participants in this study received their PTDI certificates.

Participants in the conventional training group completed the scheduled classroom instruction and BTW curriculum, while the simulator training group completed the scheduled classroom instruction along with the split BTW and simulator curriculum at DTCC. A total of 15 training sessions were completed to obtain the number of participants needed for the conventional and simulator training groups. Each session had a maximum of six students (three conventional students, three simulator students) with two road instructors and two range instructors (for a student-instructor ratio of 3:1). This 3:1 ratio met PTDI's standards for student to instructor ratio. For the duration of the 8-week program, each student was trained by the same road instructor and range instructor. Thus, the same road and range instructors trained the conventional group for the entire 8-week course, and the same road and range instructors trained the simulator group for the entire 8-week course. The experimental design counter-balanced each of the instructors to train an equal number of students between the two groups. While training students in the simulator group, the same instructors provided both BTW and simulator training. The instructors followed the same road and range course syllabi, objectives, and lesson plans while training students in the simulator. These instructors trained and "coached" the students just as they would in the real truck; however, the additional features available through simulation (e.g., overhead view and reply) were used. Additionally, no training or "coaching" was provided on testing days for either BTW or simulator. Only route directions (i.e., road test) and testing procedures were explained to the students. Sessions lasted for 8 weeks and classes were held 5 days a week. A summary of the DTCC curriculum is provided in appendix B. There were a total of 32 lesson plans, each lasting approximately 6 hours, with an equal number of lessons between road and range driving. The instructors were dedicated to either road or range instruction and did not switch between the two.

Under the CTP training program, the range-driving class Commercial Transportation Studies (CTS) 105 covered all 8 weeks. The road driving consisted of two different courses, CTS 104 and CTS 107. For the first 4 weeks, the trainees were enrolled in CTS 104, which taught basic operating practices including vehicle control and shifting. During the second 4 weeks, the trainees were enrolled in CTS 107; this course trained participants in advanced operating practices including proper communication, safe operating practices, speed management, and visual search techniques. Figure 4 illustrates the timeline for the three BTW classes comprising the 8-week curriculum. It should be noted the simulator group also followed this timeline; however, 58 percent of their driving time per course was conducted in the simulator. Participants in both the conventional and simulator groups also were enrolled in four classroom-based classes teaching vehicle systems, driving theory, log books, and Federal Regulations. 


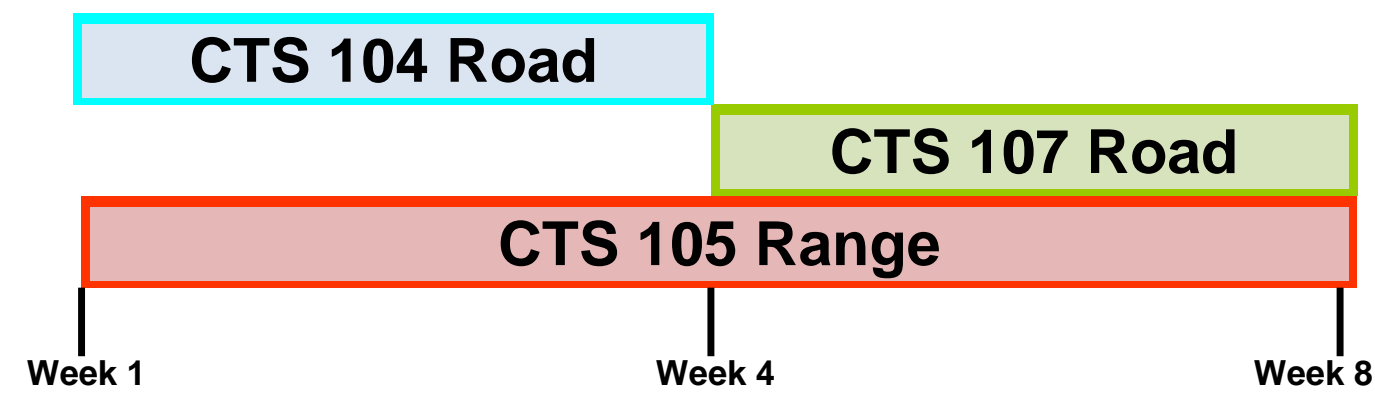

Figure 4. Diagram. DTCC CTS BTW curriculum schedule.

\subsubsection{CDL-focused and Informal Training Groups}

The CDL-focused trained participants were entry-level Class A CDL test-takers who received training at a CDL-focused truck driver training school. This type of training normally involved a compressed (typically 2-4 weeks) training schedule. There are no minimum standards or required number of BTW training or classroom hours. This training typically consisted of 1-3 days of classroom training associated with learning the CDL manual to obtain their Class A CDL learner's permit. The number of BTW training hours showed a wide variance between participants. This method of training typically reported a student-instructor ratio of 5:1.

Participants in the informal training group consisted of entry-level Class A CDL test-takers who had not received formal Class A CMV driver training. The training and practice that these participants received was not standardized and did not occur through any of the other three methods of training previously discussed. These participants may have learned to drive a tractortrailer through family members, friends, co-workers, or simply through trial and error on their own. No classroom training occurred for the informal training group.

\subsubsection{Data Acquisition System}

The DTCC CTP used four trucks for road and range instruction and testing. Each of these trucks was instrumented with a data acquisition system (DAS), as shown in Figure 5. In addition, a DAS was used to collect data from the driving simulator network.

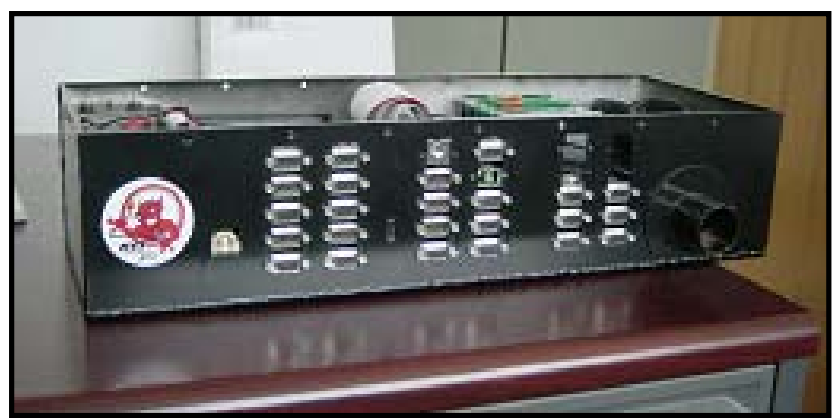

Figure 5. Photo. The DAS.

The DAS is a centralized data collection device that has been successfully used in a number of naturalistic driving studies (Blanco, Hickman, Klauer, \& Hanowski, 2006). This system consists of a microcomputer that receives, processes, and stores data from modular sensors positioned 
throughout the vehicle. Besides being configured as a modular system, the DAS sensor array operates in a protected fashion; any single sensor failure does not stop data collection from any other sensor in the network. Onboard storage is via removable hard drive; data were collected at the conclusion of each day's activities. The DAS unit was mounted unobtrusively underneath the rear bench seat of the truck cab and was not visible to the driver, or passengers.

The DAS is powered through the truck's alternator; an additional power source is provided through a back-up battery located in the DAS. The DAS turns on with the ignition and collects data continuously at 10 Hertz until the vehicle engine has been shut down for 3 minutes. Four video cameras are installed on each truck to provide a wide angle of the truck cab and driver's face, the forward-facing view out of the windshield, and the view down the each side of the truck (from two cameras).

A second DAS collected information from the simulator. Besides collecting equivalent sensor data from the simulator network, the video recording on the simulator DAS was configured to record views similar to those recorded in BTW driving.

\subsubsection{Driving Simulator}

A FAAC, Inc., model TT-2000-V7 driving simulator was selected for use in this study (Figure 6). This simulator provides a 225-degree seamless (borderless screens) forward field of view with five forward visual channels.

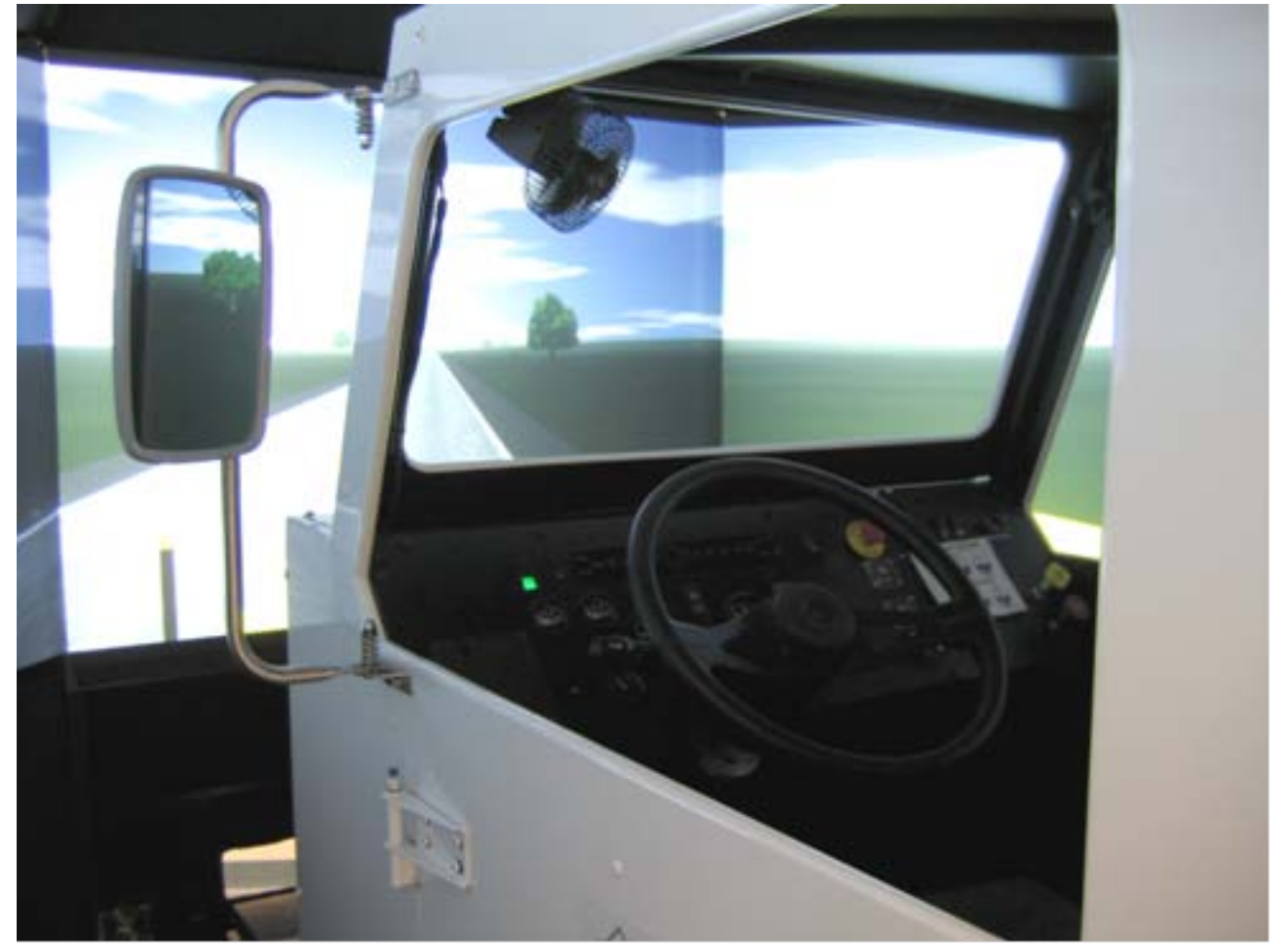

Figure 6. Photo. The TT-2000-V7 Driving Simulator

Two rear visual channels provide views through the use of real mirrors (Figure 7). Multiple transmission configurations, different trailer length settings, and multiple engine configurations are also provided. These requirements were established during earlier SimVal research. 


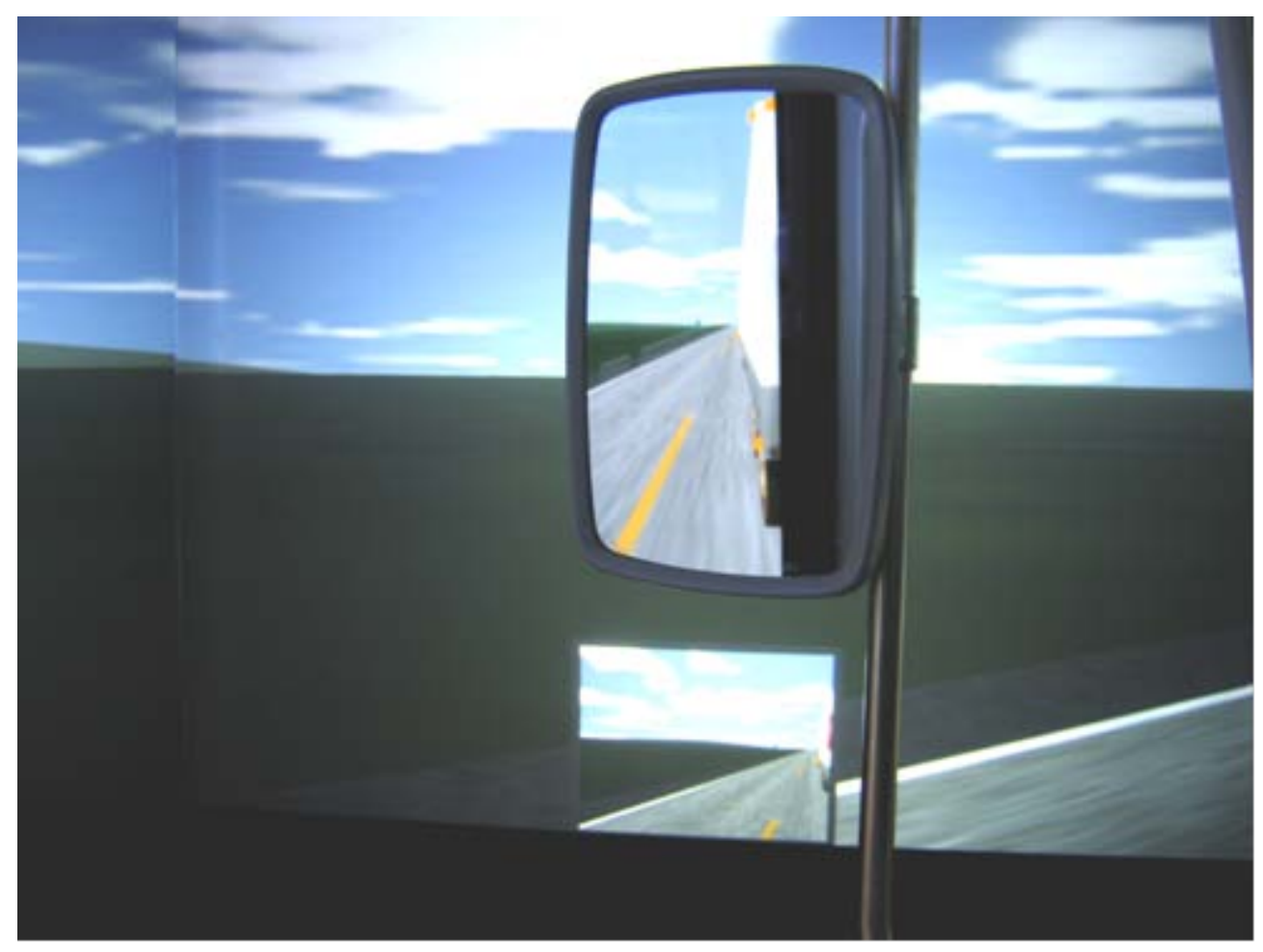

Figure 7. Photo. The Rear Visual Channel as Viewed Through the Simulator Mirrors.

\subsubsection{Driving Simulator Scenarios}

\subsubsection{Orientation Scenarios}

Simulator pre-screening and orientation to the simulator cab were identical for all participants. The orientation began with an introduction to the features of the simulator, including the seat belt, seat adjustments, steering, pedals, parking brake, gauges, switches, and other features (see appendix C). After orientation to the cab, the participants drove an orientation scenario route designed to allow the participant to get accustomed to the simulator's controls and a feel for the simulated vehicle dynamics. For this drive, participants were given a truck with no trailer (i.e., a bobtail configuration) with an automatic transmission. This driving route began on a rural twolane road with minimal visuals and proceeded to the interstate and ended in town. No traffic was present during this orientation drive. The same route was driven by all participants and lasted for 30 minutes. Upon completion of this simulator orientation drive, participants completed a postdrive simulator sickness questionnaire to assess the participant for any discomfort or motion sickness after 30 minutes of simulator driving (appendix D). Participants were also allowed to take a break at this time. Participants who reported or showed visible signs of discomfort based on a modified version of the Simulator Sickness Questionnaire (Kennedy et al., 1993) were instructed to stop their activities immediately and were not allowed to continue using the simulator.

Those participants who did not report or show visible signs of simulator discomfort were allowed to continue with a second orientation drive. This orientation route, approximately 30 minutes in length, was a free drive where the participants could drive wherever they liked and included interstate highways, rural 2-lane roads with winding curves and hills, and city driving in light 
traffic. A tractor with an automatic transmission and no trailer was used for the conventional and simulator groups as these participants had no prior experience driving a tractor trailer. However, the informal and CDL-focused training groups were provided the same trailer and transmission configuration they used for testing. At the end of the second 30 minute orientation drive, the participants were asked to complete another simulator post-orientation questionnaire (appendix F) and were allowed to take another break.

Any DTCC CTP student who exhibited simulator discomfort was assigned to the conventional group, as all training received in this group was BTW. Also note that any participant in the informal or CDL-focused groups who experienced simulator sickness was allowed to continue participation; however, only BTW testing was conducted (and no simulator testing).

\subsubsection{Entry-Level Training Scenarios}

The simulator training group used scenarios created to match as closely as possible existing DTCC CTS program daily lesson plans. The vehicle dynamics on the simulator were changed depending on the corresponding DTCC daily lesson plan. During the first 4 weeks of road training (CTS 104), participants drove a 9-speed double-clutching transmission with a 40-foot, empty van trailer. In the last 4 weeks of road training (CTS 107), students drove both 9-speed and 10-speed double-clutching transmissions. The students also switched between a half-loaded 45-foot flatbed and a half-loaded 48-foot van trailer. Additionally, the students received 8 weeks of range driving (CTS 105). On the range, a 9-speed double-clutching transmission with an empty 40-foot van trailer was used.

The simulated range and road tests were developed to replicate the Delaware DMV road/range test, while the daily simulated training scenarios were developed to replicate as closely as possible the BTW driving in the DTCC CTP lesson plans. These routes, while parallel in structure, included features specific to the Simulator training, including roadside wildlife (both stationary and moving), pedestrians (both stationary and moving), bicyclists and motorcycle riders, weather conditions, construction zones, crash scenes, and emergency equipment. In addition to the autonomous vehicles, programmed traffic events were added, including vehicles running a stop sign, unexpectedly backing out of driveways, pulling onto the roadway from the shoulder, and passing oncoming traffic in a direction facing the simulator driver.

\subsubsection{Advanced Capabilities Showcase Scenarios}

The ACS study assessed the validity of simulator performance by replicating emergency maneuvers, extreme road conditions, and different trailer types (including van trailers, tanker trailers, and doubles). Additionally, this exploratory study assessed the procedures of rating driver performance for the emergency maneuvers and extreme driving conditions, simulator discomfort, and simulator programming of these events. In the ACS study, a comprehensive 75minute scenario was developed covering emergency maneuvers and extreme driving conditions (see appendix E for operational definitions of the emergency maneuvers and extreme conditions). All events were introduced as part of a normal drive. Emergency events encountered along the drive included merge squeezes, lane crosses, steering axle tire blowouts, potential rollovers, emergency braking, brake failures, off-road recoveries (evasive action), animal crossings, blind entering vehicles, pedestrians, and tight city turns. Extreme conditions included fog, heavy rain with slick roads, heavy snow with slick roads, black ice, 8 percent upgrades, 8 
percent downgrades, dirt roads, construction zones with lane closure, roadway obstructions, and railroad crossings.

\subsection{MEASURES}

\subsubsection{Performance Measures}

\subsubsection{Driving Records}

Participants' previous personal driving records as well as their record as a driver where a Class A CDL was needed to drive were recorded at 4 and 12 months after employment. Databases examined included the Delaware DMV and FMCSA's Commercial Driver's License Information System (CDLIS). Information collected through these systems included the date and type of the citation or violation, the violation code, officer comments, whether or not a crash was involved, the State where the violation occurred, whether or not a CMV was involved in the incident (if this information was missing from the police accident report, the default was private vehicles only), and any points assessed to the driver's license. Due to the potential for a longer period of time between citation and conviction, citations were collected by the date of citation rather than by conviction date in an effort to minimize missed citations.

\subsubsection{Knowledge, Road and Range Tests}

Participant DMV knowledge and road and range test records were obtained. Knowledge test records provided the number of attempts taken to pass the general, air brakes, and combination vehicle tests. Participants' road and range scores were also obtained which provided a percentage grade. The DTCC and simulator road and range tests were scored using the same criteria as the Delaware DMV tests, and the tests were both administered using the American Association of Motor Vehicle Administrators (AAMVA) CDL examiner's manual. However, the DMV tests only included a random selection of three of the range maneuvers. The test and other dependent variables related to these tests are provided in appendix F.

\subsubsection{Advanced Capabilities Showcase Scoring}

Each group of ACS participants experienced various emergency maneuvers (e.g., front tire blowout, emergency stop, brake failure, off-road recovery, animal crossing) and extreme conditions (e.g., heavy rain, snow-covered roads, black ice, steep upgrades/downgrades, construction zone) during their participation in the ACS study. Emergency maneuvers and extreme conditions were broken down into categories of traffic/road events and mechanical failure. The experimenter scored the participant's response to each emergency maneuver and extreme driving condition. Each response was classified as "responded appropriately," "responded inappropriately," or "failed to respond," depending on the driver's response to the situation.

\subsubsection{Subjective Measures}

\subsubsection{Modified Simulator Sickness Questionnaire}

A modified version of the Simulator Sickness Questionnaire (Kennedy et al., 1996) was used to assess participants' subjective ratings of discomfort from simulator exposure. This measure 
consisted of 17 symptoms which the participants rated on a scale from " 0 " (not experiencing the symptom) to " 3 ” (experiencing severe levels of the symptom). The questionnaire is provided in appendix D.

\subsubsection{Entry-level 4- to 5-Month Self-Assessment}

A self-evaluation questionnaire was conducted over the telephone with each participant at 4-5 months after employment. These questions contributed to the collection of dependent variables in the follow-up evaluation. They sought to provide additional insight into the trainees' perceptions and opinions of how they rate their training in terms of basic vehicle control and maneuvering, safe operations, and validity of the simulated CDL test as compared to their peers. In addition, job satisfaction was evaluated at this point (see appendix G for the specific selfassessment questionnaire).

\subsubsection{Entry-level 4-5-Month Supervisor Assessment}

A supervisory evaluation was obtained over the telephone from supervisors who agreed to participate for each of those participants at 4-5 months after employment. The questions allowed the research team to gain additional information about the trainee in terms of miles driven since hire, number of USDOT-reportable and non-USDOT-reportable crashes, number of moving violations, rating of the trainee on basic vehicle control, maneuvering skills, and safe operations as compared to the drivers' peers. See appendix $\mathrm{H}$ for the specific “Commercial Driver On-theJob Safety Performance Employer Questionnaire.”

\subsubsection{Advanced Capabilities Showcase Questionnaire}

ACS participants completed a questionnaire asking about their years of driving experience, license status and endorsements, training history, and experience with hazardous/dangerous situations during their driving career (appendix I). Also a post-simulator drive questionnaire also asked drivers to assess features between the simulator and an actual truck (i.e. mirrors, upshifting), provide ratings on the recreated simulator events encountered while driving the simulator, and provide comments including what they liked most and least about the simulator. The full questionnaire is provided in appendix $\mathrm{J}$.

\subsubsection{Aspects of the Simulator Ranking}

Participants in the simulator training group and the ACS sub-study were provided with a list of 10 aspects of the simulator (e.g., safety, overhead views, repeatability, standardization, realism, enrichment, basic control, vehicle handling, safe operations (defensive driving), and backing skills). Table 4 provides the operational definitions for each of these aspects. Theses participants were asked to rank each of the aspects as effective or not effective. 
Table 4. Operational Definitions of Ranking Aspects

\begin{tabular}{|l|l|}
\hline \multicolumn{1}{|c|}{ Ranking Aspect } & \multicolumn{1}{c|}{ Operational Definition } \\
\hline Safety & $\begin{array}{l}\text { No physical harm to the driver, trainer/examiner, and no vehicle or property } \\
\text { damage if an incident occurs. }\end{array}$ \\
\hline Overhead Views & Ability of the simulator to provide bird's eye view for instruction/training. \\
\hline Repeatability & Ability to have the same event/condition over and over for practice \\
\hline Standardization & $\begin{array}{l}\text { Ability to provide to same driving conditions/events to each driver (i.e., non- } \\
\text { standard = in the real truck one driver may encounter heavy traffic and } \\
\text { construction while another may have rain and slick roads but light traffic). }\end{array}$ \\
\hline Realism & Ability to provide a realistic driving experience. \\
\hline Enrichment & $\begin{array}{l}\text { The use of the simulator to expose drivers to special conditions such as } \\
\text { weather events, mountainous terrain, etc. }\end{array}$ \\
\hline Basic Control & $\begin{array}{l}\text { The use of the simulator for initial or refresher training of basic truck control } \\
\text { skills such as shifting, starting, and stopping. }\end{array}$ \\
\hline Vehicle Handling & $\begin{array}{l}\text { The use of the simulator for initial or refresher training of vehicle handling } \\
\text { skills such as proper communication, speed maintenance, etc. }\end{array}$ \\
\hline Safe Operations/Defensive Driving & Ability of the simulator to teach/refresh defensive driving techniques \\
\hline Backing Skills & $\begin{array}{l}\text { The use of the simulator for initial or refresher training of the CDL backing } \\
\text { maneuvers. }\end{array}$ \\
\hline
\end{tabular}

\subsection{PROCEDURE}

\subsubsection{Sample Determination and Power Analysis}

Prior to the start of the current effort, an exploratory study assessed the simulator programming and procedures to be used in the entry-level training study. Based on the result of this power analysis, 21 participants per group were needed to reach a statistical power of 0.8 .

\subsubsection{Entry-Level Training Study}

After recruitment and obtaining informed consent, participants completed a simulator orientation (including information on the various controls and adjustments of the simulator) and two orientation drives. Each orientation drive was followed by an assessment of simulator sickness symptoms.

After orientation, participants in the conventional and simulator training groups completed normal training in the DTCC CTP program. Participants in the simulator group received 58 percent of their total vehicle training time in the driving simulator. Conventional group participants received 100 percent of their total vehicle training time in real trucks. At the midpoint of their training session, participants in both groups completed a mid-term road test on the simulator and truck (DTCC test). At the conclusion of the training session, participants in both groups completed range and road tests on the simulator and truck (DTCC test). Participants in the informal and CDL-focused training groups received DTCC and simulator road and range tests (identical to the final tests taken by the Conventional and simulator groups).

Scores on three versions of road and range tests were obtained for each participant. These include: simulator road and range tests, DTCC road and range tests, and DMV road and range 
tests. Road and range tests, as well as testing modalities (DTCC or simulator), were counterbalanced.

Follow-up occurred at 4-5 months after being employed as a Class A CDL driver. At this time, participants completed a self-assessment and a replication of their final assessment testing (including road and range DTCC and simulator evaluations). Participants' supervisors were contacted and asked to provide an assessment on the participant-driver's performance.

\subsubsection{Advanced Capabilities Showcase Study}

After recruitment and obtaining informed consent, participants completed a simulator orientation (including information on the various controls and adjustments of the simulator) and two orientation drives. Each orientation drive was followed by assessment of simulator sickness symptoms. After completion of the simulator orientation, participants began with the actual showcase scenario. The trailer type selected was dependent on the trailer the participant currently pulls at his/her place of employment. A conventional truck with 10-speed double clutching transmission was used in conjunction with either a van, tanker, or a doubles trailer unit. The load depended on the trailer type.

Participants in the ACS sub-study then completed a comprehensive simulator scenario which included both emergency situations and extreme conditions (full descriptions are provided in the Advanced Capabilities Showcase Scenarios section, above). The experimenter provided verbal driving directions while scoring the participant. The experimenter only announced which roads to take and did not cue the driver to any pending events.

The participant experienced different emergency situations and extreme conditions along the drive. In the event of an incident or crash, the experimenter had a remote control that allowed the scenario to be restarted at the point 30.0 seconds (s) before the incident or crash occurred. At the completion of the ACS scenario, the participant stopped and parked the simulated vehicle and exited the simulator. The participant then completed a questionnaire about the simulated drive. 
[This page intentionally left blank.] 


\section{RESULTS}

\subsection{INTRODUCTION AND OVERVIEW}

The following are the findings of an examination of the training methods evaluated in the current research effort, with respect to the research questions stated in Section 3. Any specific methods used (beyond those detailed in the method section in Section 4), results obtained, and a brief discussion of each major research question are presented in this chapter. This chapter includes all study topics, including results of the examination of entry-level training (Topics 1-7) and the Advanced Capabilities Showcase (ACS; Topics 8 and 9).

\subsection{TOPIC \#1: EFFECTS OF TRAINING METHODS ON CDL AND RELATED TEST PERFORMANCE}

\subsubsection{Research Question 1a: Does the training method affect DMV road/range test performance?}

This research question addressed the effect of training method in the skills measure of driver performance; specifically, whether or not training method affected DMV road and range test performance. The hypothesis for this research question predicted that training will have a beneficial effect on driver licensing performance, and that participants receiving simulationbased training will obtain similar or higher scores on the DMV road/range test than participants receiving conventional, CDL-focused, or Informal training. Based on the completeness of the curriculum followed by the conventional and simulator groups, it was expected that driver skills will be greatest for the conventional and simulator-trained students, less for the CDL-focused training students, and least for the students with no formal training.

\subsubsection{Method and Results}

Data were collected for participants' DMV road and range test scores. Due to lower than expected Class A CDL throughput in Delaware, data collection was expanded to nearby Maryland DMV locations. A total of 12 DMV test scores, nine participants from Maryland and three participants from Delaware, were unable to be obtained. Thus, only the DTCC and simulator range and road test scores were available for these 12 participants.

It should be noted that there was a 3-week delay between the time a driver completed DMV testing and the test scores were posted in the DMV database system (note that this process was changed in the last months of data collection, with test results being made available the day after testing occurred). However, per the study procedures, participants were required to complete the initial testing within 3 weeks of receiving their Class A CDL, but before being employed to use a Class A CDL, to ensure there was no degradation in their acquired skills due to a lack of driving time. All drivers passed the DMV road test on the first attempt. Figure 8 displays the number of attempts to pass the DMV range test across groups. As shown in Figure 8, 89 percent of all participants passed the DMV range test on the first attempt, 8 percent required a second attempt, and 2 percent required a third attempt to pass the range test. Each driver's score on the final passing attempt was used in the analysis shown in Figure 9. 


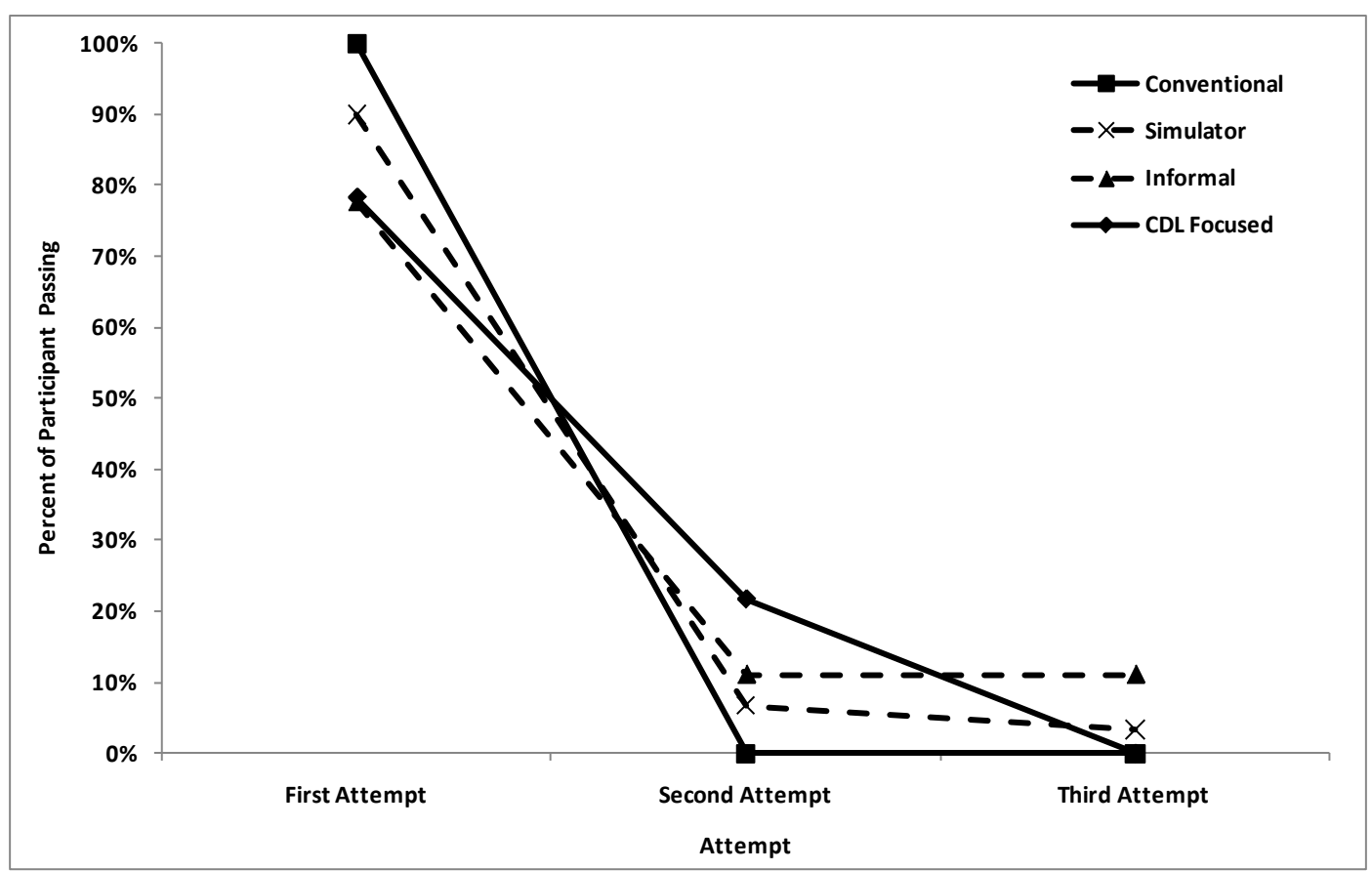

Figure 8. Graph. Attempts taken to pass DMV range test, by group.

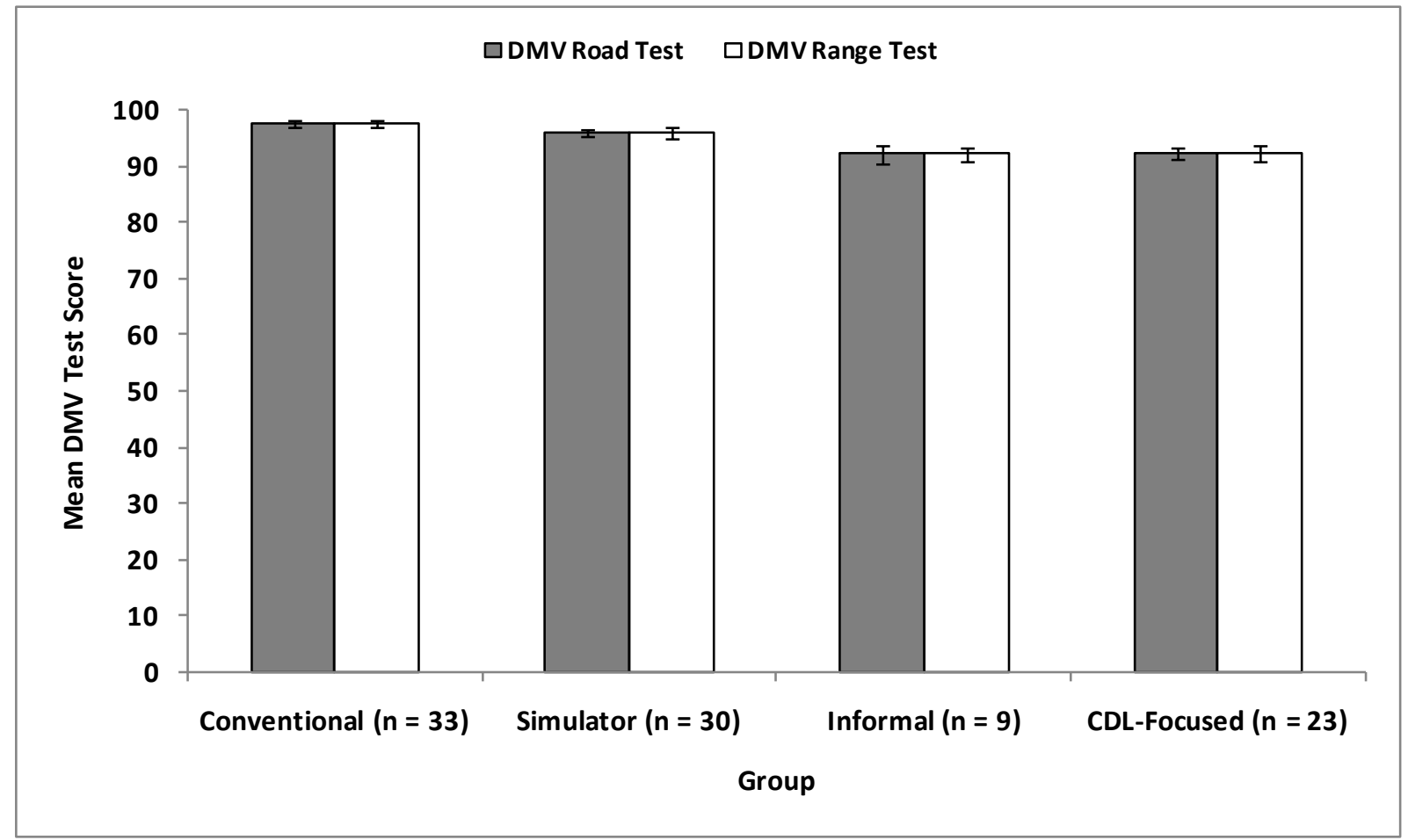

Figure 9. Chart. DMV road and range scores, by group

Error bars represent standard error.

Figure 9 displays the mean scores and standard deviations for the DMV road and range tests across all groups. In all cases the mean DMV road and range test scores were above 90 percent, regardless of group membership. 
A multivariate analysis of variance (MANOVA) was performed on the DMV road and range tests. Note that the MANOVA used only observations where all of the individual tests had results (scores present in both DMV road and range tests). Using Wilks' criteria (lambda $=0.83$ ), the MANOVA was significant, $F(6,180)=2.86, p=.01$; thus, analysis of variance (ANOVA) tests were performed on the DMV road and range tests. The ANOVA for the DMV road test found no significant group differences between groups $F(3,91)=1.94, p=0.13$. However, the ANOVA for the DMV range tests found significant difference between groups, $F(3,91)=5.49, p=0.002$.

Planned comparison analyses using Bonferroni-corrected t-tests were performed on the DMV range tests. The Bonferroni-corrected $t$-test was chosen as a more conservative method of analysis. Results indicated two significant differences between group means on the DMV range test. Participants in the conventional training group (97.6 percent) scored significantly higher than participants in the CDL-focused (92.4 percent) and Informal (92.2) Training Groups. Table 5 shows the difference scores and 95 percent confidence limits for all pair-wise tests on the DMV range test.

Table 5. Simple Effects Tests for DMV Range Tests

\begin{tabular}{|l|c|c|c|}
\hline \multicolumn{1}{|c|}{ Comparison } & $\begin{array}{c}\text { Difference between } \\
\text { Means }\end{array}$ & $\begin{array}{c}\text { Lower 95\% } \\
\text { Confidence Limit }\end{array}$ & $\begin{array}{c}\text { Upper 95\% } \\
\text { Confidence Limit }\end{array}$ \\
\hline Simulator-Conventional & 1.74 & -1.85 & 5.33 \\
\hline Simulator-Informal & 3.64 & -1.80 & 9.06 \\
\hline Simulator-CDL-focused & 3.43 & -0.52 & 7.38 \\
\hline Conventional-Informal & $5.38^{*}$ & 0.03 & 10.74 \\
\hline Conventional-CDL-focused & $5.17^{*}$ & 1.30 & 9.04 \\
\hline Informal-CDL-focused & -0.21 & -5.81 & 5.39 \\
\hline
\end{tabular}

${ }^{*} p<0.05$

In order to ensure that the use of only the last, passing, DMV test scores did not obscure the true differences between groups, the DMV scores were adjusted based on the number of times taken to pass the test. Participants who required multiple attempts to pass the DMV range test had a new score calculated averaging their passing score with prior failures (set to a value of 50 points). Thus, a participant passing the DMV range test on the third attempt with a score of 100 would have a new, adjusted score of 66.6 (reflecting the average of two failing grades of 50 and the final passing grade of 100). As indicated above, all participants passed the DMV road test on the first attempt; thus, this adjustment was only performed on DMV range tests. The original and adjusted mean DMV range scores are shown in Figure 10. 


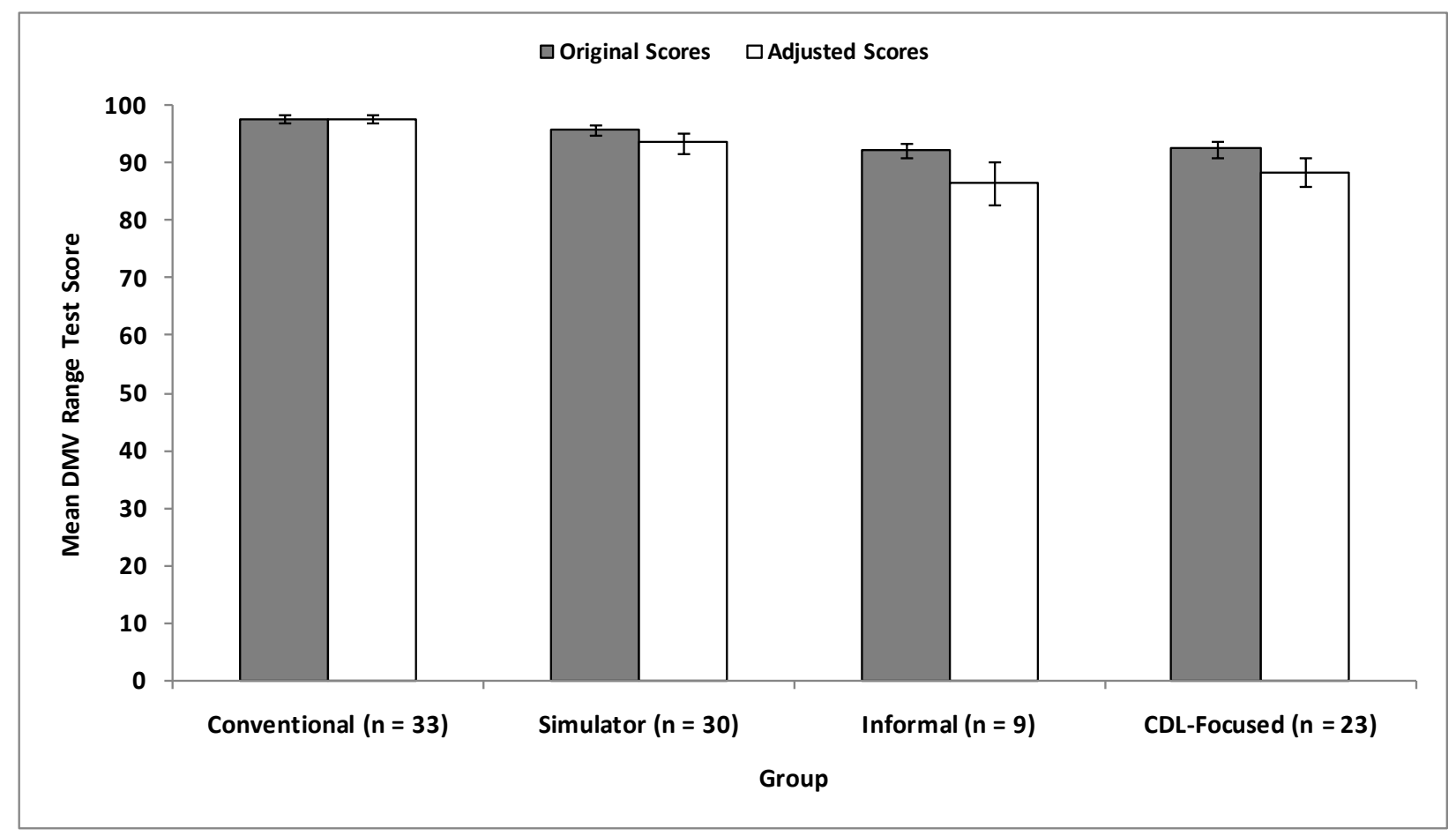

Figure 10. Chart. Original and adjusted mean DMV range scores, by group.

Error bars represent standard error.

These adjusted scores were analyzed using an ANOVA. The ANOVA for the adjusted DMV range test found a significant effect for group, $F(3,94)=6.44, p=.0005$. Post-hoc examination via Bonferroni-corrected $t$-tests indicated the conventional training group (97.6 percent) scored significantly higher than the CDL-focused (88.4 percent) and informal training group (86.6 percent). There was no difference indicated between the conventional and simulator (93.6 percent) training groups, and no other comparison reached statistical significance. These results indicate the same statistical relationship as was found using the original, unadjusted scores. Thus, no further analysis was conducted using the adjusted scores.

Tests similar to the DMV CDL test were conducted in both DTCC (i.e., road and range tests performed in real vehicles) and simulator modes. Both the DTCC and simulator road and range tests scored using the criteria, and administered using the same standard (the AAMVA CDL examiner's manual) as the DMV tests. Further, as the DTCC instructors scored every DTCC and simulated road and range test, a sample of DTCC and simulator tests from each training group were scored by an independent reviewer. This was conducted to ensure that the DTCC instructors were not biased in the grading of the tests (i.e., to conform to the research hypotheses and/or unfairly grade drivers that were not enrolled in the DTCC curriculum). The results of this validation are provided in appendix $\mathrm{K}$, and indicated that no bias was present in instructor scoring of the tests.

A MANOVA was performed on the DTCC and simulator road and range tests (using only observations where all of the individual tests had results). Using Wilks' criteria (lambda $=0.18$ ), the MANOVA was significant, $F(12,243.7)=18.93, p<.0001$; thus, ANOVA tests were 
performed on the DTCC and simulator road and range tests. The results of these ANOVA tests are shown in Table 6, and indicate significant group differences in all tests.

Table 6. Follow-Up ANOVA results for DTCC and Simulator Road and Range Tests

\begin{tabular}{|l|c|c|}
\hline \multicolumn{1}{|c|}{ Measure } & \multicolumn{1}{c|}{ ANOVA Result } & Outcome \\
\hline DTCC Road Test & $F(3,95)=96.62, p<.0001$ & Significant difference \\
\hline Simulator Road Test & $F(3,95)=56.80, p<.0001$ & Significant difference \\
\hline DTCC Range Test & $F(3,95)=18.43, p<.0001$ & Significant difference \\
\hline Simulator Range Test & $F(3,95)=15.99, p<.0001$ & Significant difference \\
\hline
\end{tabular}

Bonferroni-corrected t-tests between group scores on the DTCC road test indicated that both the conventional and simulator training groups had significantly greater scores on the DTCC road test as compared to both the Informal and CDL-focused training groups. There was not, however, a difference between the conventional and simulator training groups, or between the Informal and CDL-focused training groups. When the Simulator road tests were examined, similar overall patterns were present. The Bonferroni-corrected t-tests indicated that both the conventional and simulator training groups scored significantly greater on Simulator road tests as compared to both the CDL-focused and informal training groups. No differences were present between either conventional and simulator groups, or informal and CDL-focused groups. The mean score for each group on both DTCC and Simulator road tests is displayed in Figure 11. These results will be further examined in the research questions of Topic 2 (Validity of Simulation Training and Testing).

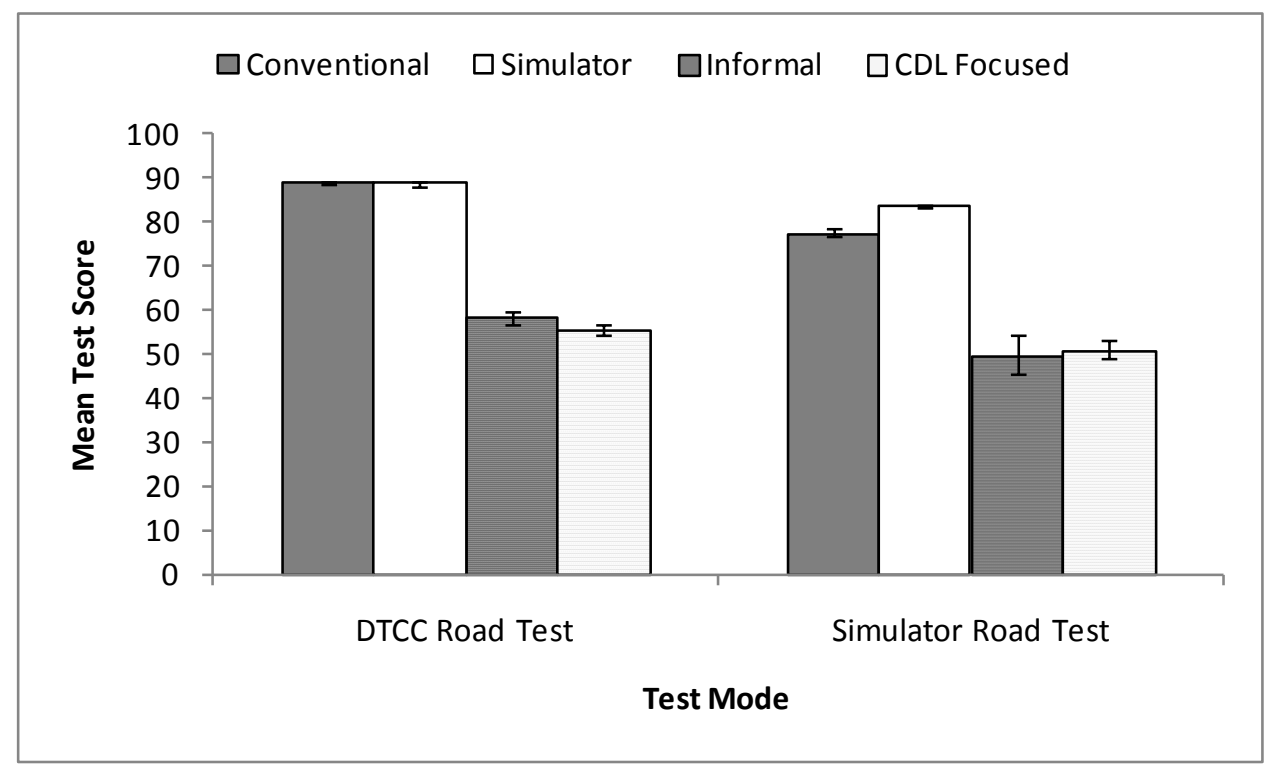

Figure 11. Chart. Mean DTCC and Simulator road test scores, by group. Error bars represent standard error.

Bonferroni-corrected $t$-tests between group scores on the DTCC range test indicated that both the conventional and simulator training groups had significantly greater scores on the DTCC range test as compared to the CDL-focused group. Additionally, the informal group had a significantly greater score than the CDL-focused group. When the simulator range tests were examined, a 
different pattern of results was present. The Bonferroni-corrected $t$-tests indicated the simulator group had significantly greater scores on the simulator range test as compared to the conventional, informal, and CDL-focused groups. No other comparison reached significance. The mean score for each group on both DTCC and simulator range tests is given in Figure 12. These results will be further examined in the research questions of Topic 2 (Validity of Simulation Training and Testing).

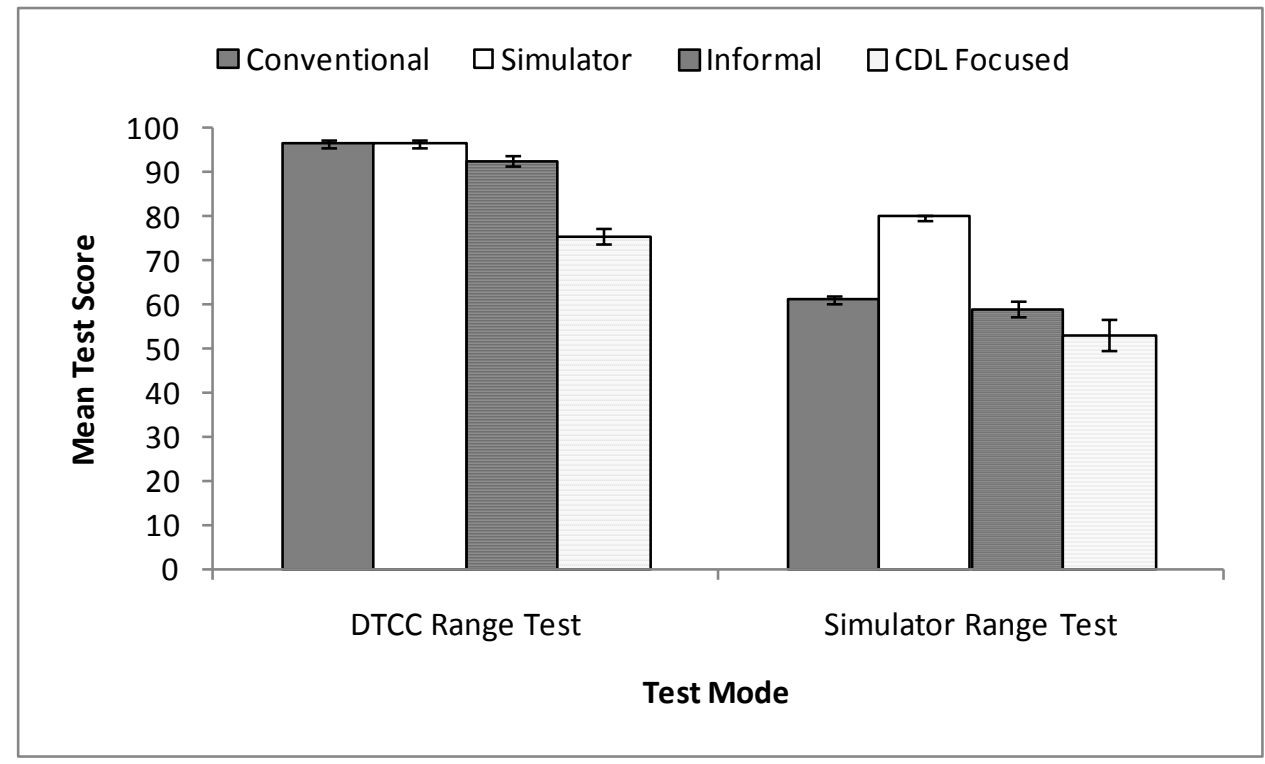

Figure 12. Chart. Mean DTCC and simulator range test scores, by group.

Error bars represent standard error.

\subsubsection{Discussion}

Two primary issues were evaluated in this research question:

Simulator students' capability to perform as well as students trained by the other methods.

- Non-certified methods’ performance compared to the certified methods’ performance.

First, the results suggest that DTCC participants who received part of their training in a truck simulator do not significantly differ in test performance as compared to DTCC participants who received all of their training BTW (i.e., conventional).

Further, when looking at the different training methods, there were statistical differences in the DMV range test results, but no difference in the DMV road test. On average, all training methods passed the DMV road and range tests with an average score above 90; thus, these differences are not considered practical differences.

However, when analyzing the scores on DTCC and simulator versions of the DMV tests (scores that were validated by an independent, external, reviewer), there are significant group differences present. In DTCC testing, the conventional and simulator training groups almost always outperformed both the informal and CDL-focused training groups. In simulator tests, the simulator group consistently outperformed both Informal and CDL-focused groups. The 
statistical differences suggest that students trained in a PTDI-certified program, on average, scored better than the drivers that did not undergo a certified training program in these tests.

\subsection{TOPIC \#2: VALIDITY OF SIMULATION TRAINING AND TESTING}

Four research questions were addressed in this topic:

Research Question 2a: Can simulation testing adequately assess road/range driving skills?

Research Question 2b: What is the correlation between scores from the DTCC road/range test and its simulated counterpart?

Research Question 2c: Which test scores (DTCC or simulated) are more predictive of subsequent DMV tests?

Research Question 2d: What features of the simulator will be most and least effective for participants primarily trained on the simulator?

The method, analysis, measures of interest, results, and discussion related to these questions are presented below.

\subsubsection{Method}

Each participant's scores on three versions of road and range tests were obtained. These include: simulator road and range tests, DTCC road and range tests, and DMV road and range tests. In some cases, participant scores were not available from the DMV testing office. Thus, some participants were excluded from the analyses involving DMV scores. Participants who did not take a specific test (e.g., participants who demonstrated symptoms of simulator sickness did not complete the Simulator range and road tests) were eliminated from analysis of simulator data.

In addition, failing test scores (in either the simulator tests, or DTCC tests, or DMV tests) were all replaced with a set score of 50 percent. This was done as participants were not allowed to complete testing once they scored below 75 percent. Thus, it was unknown what their actual score would have been if they had been allowed to continue. The choice of 50 percent was made in an attempt to reduce the effect of extreme values (such as 0 percent) in the analysis while keeping a representative failing score. As discussed in the first research question, a sample of the DTCC instructor-scored DTCC and Simulator tests from each training group were scored by an independent reviewer. Analysis of the instructor and reviewer scoring indicated no systematic bias in instructor scoring (see appendix K, Test Score Validation Results).

In addition, participants' Simulator and DTCC road/range tests were recorded via DAS and subject to data reduction to evaluate safety-related events, including longitudinal acceleration (LA), time-to-collision (TTC), and lane departure (LD) events.

\subsubsection{Research Question 2a: Can simulation testing adequately assess road/range driving} skills?

This research question addressed the relationship between simulated and BTW tests of road and range driving skills. The hypothesis for this research question was that “drivers' total scores on 
the simulated road and range tests will demonstrate a statistically significant positive correlation with the DMV and DTCC road and range test scores.”

\subsubsection{Results}

Table 7 shows the means and standard deviations were calculated for each group's scores on each of the three test modalities (DTCC, Simulator, and DMV). Note that the number of participants presented in Table 7 reflect the number included in these specific analyses and could be lower than the actual total number of participants in that training group. This is because some participants did not take the Simulator test due to simulator sickness. For example, out of the 33 conventional training group participants' results, 29 were available for the Simulator road test (4 participants experienced simulator sickness and were not able to perform the test). Additionally, out of the 33 CDL-focused group participants' results, 23 are available for the DMV tests (3 participants' test scores were lost by the Delaware DMV, and 7 participants were tested at the Maryland DMV which did not keep records of the test scores). 
Table 7. Descriptive Statistics for Each Group's Test Scores

\begin{tabular}{|l|l|r|r|r|r|r|}
\hline \multicolumn{1}{|c|}{ Measure } & Training Group & \multicolumn{1}{c|}{$\boldsymbol{n}$} & Mean (\%) & SD (\%) & $\begin{array}{c}\text { Minimum } \\
\text { (\%) }\end{array}$ & $\begin{array}{c}\text { Maximum } \\
\text { (\%) }\end{array}$ \\
\hline DMV Range Test & Conventional & 33 & 97.61 & 3.90 & 83 & 100 \\
\hline DMV Range Test & Simulator & 30 & 95.87 & 5.25 & 82 & 100 \\
\hline DMV Range Test & Informal & 9 & 92.22 & 3.38 & 86 & 96 \\
\hline DMV Range Test & CDL-focused & 23 & 92.43 & 7.26 & 76 & 100 \\
\hline DMV Road Test & Conventional & 33 & 98.30 & 2.64 & 90 & 100 \\
\hline DMV Road Test & Simulator & 30 & 97.80 & 3.00 & 87 & 100 \\
\hline DMV Road Test & Informal & 9 & 95.33 & 4.61 & 89 & 100 \\
\hline DMV Road Test & CDL-focused & 23 & 96.87 & 4.88 & 80 & 100 \\
\hline DTCC Range Test & Conventional & 33 & 96.73 & 5.23 & 76 & 100 \\
\hline DTCC Range Test & Simulator & 32 & 96.50 & 3.83 & 87 & 100 \\
\hline DTCC Range Test & Informal & 9 & 93.00 & 5.55 & 83 & 100 \\
\hline DTCC Range Test & CDL-focused & 33 & 75.79 & 20.67 & 50 & 100 \\
\hline DTCC Road Test & Conventional & 33 & 89.09 & 5.49 & 75 & 99 \\
\hline DTCC Road Test & Simulator & 32 & 88.90 & 0.06 & 77 & 98 \\
\hline DTCC Road Test & Informal & 9 & 58.56 & 12.85 & 50 & 77 \\
\hline DTCC Road Test & CDL-focused & 33 & 55.61 & 12.25 & 50 & 88 \\
\hline Simulator Range Test & Conventional & 29 & 61.10 & 14.64 & 50 & 87 \\
\hline Simulator Range Test & Simulator & 32 & 79.94 & 19.93 & 50 & 100 \\
\hline Simulator Range Test & Informal & 9 & 59.11 & 18.09 & 50 & 92 \\
\hline Simulator Range Test & CDL-focused & 29 & 53.21 & 9.77 & 50 & 88 \\
\hline Simulator Road Test & Conventional & 29 & 77.52 & 13.63 & 50 & 95 \\
\hline Simulator Road Test & Simulator & 32 & 83.72 & 13.81 & 50 & 97 \\
\hline Simulator Road Test & Informal & 9 & 50.00 & 0.00 & 50 & 50 \\
\hline Simulator Road Test & CDL-focused & 29 & 51.07 & 5.76 & 50 & 81 \\
\hline As & & & & 50 & \\
\hline
\end{tabular}

As discussed in Research Question 1, a MANOVA was performed on the DTCC and Simulator road and range tests. As the MANOVA provided a significant result $(F(12,243.7)=18.93, p<$ .0001 , lambda $=0.18$ ), follow-up testing was conducted via ANOVA tests. These tests suggested that there were significant group differences for both the Simulator road $(F(3,95)=56.80, p<$ $.0001)$ and range $(F(3,95)=15.99, p<.0001)$ tests. Bonferroni-corrected $t$-tests revealed that the Simulator road test scores for the conventional and simulator groups were significantly greater than the scores for the Informal and CDL-focused groups. In simulator range tests, Bonferroni-corrected $t$-tests revealed that the simulator group had significantly greater scores than any of the other three groups (Table 8). 
Table 8. Simple Effects Tests for Simulator Road and Range Tests

\begin{tabular}{|l|l|r|r|r|}
\hline \multicolumn{1}{|c|}{ Test } & \multicolumn{1}{|c|}{ Comparison } & \multicolumn{1}{c|}{$\begin{array}{c}\text { Difference } \\
\text { between } \\
\text { Means }\end{array}$} & \multicolumn{1}{c|}{$\begin{array}{c}\text { Lower 95\% } \\
\text { Confidence } \\
\text { Limit }\end{array}$} & $\begin{array}{c}\text { Upper 95\% } \\
\text { Confidence } \\
\text { Limit }\end{array}$ \\
\hline Simulator Road & Simulator-Conventional & 6.20 & -1.58 & 13.98 \\
\hline Simulator Road & Simulator-Informal & $33.72^{\star}$ & 22.27 & 45.17 \\
\hline Simulator Road & Simulator-CDL Focused & $32.65^{\star}$ & 24.87 & 40.43 \\
\hline Simulator Road & Conventional-Informal & $27.52^{\star}$ & 15.94 & 39.09 \\
\hline Simulator Road & $\begin{array}{l}\text { Conventional-CDL } \\
\text { Focused }\end{array}$ & $26.45^{\star}$ & 18.48 & 34.42 \\
\hline Simulator Road & Informal-CDL Focused & -1.07 & -12.65 & 10.51 \\
\hline Simulator Range & Simulator-Conventional & $18.83^{\star}$ & 7.94 & 29.72 \\
\hline Simulator Range & Simulator-Informal & $20.83^{\star}$ & 4.80 & 36.85 \\
\hline Simulator Range & Simulator-CDL Focused & $26.73^{\star}$ & 15.84 & 37.62 \\
\hline Simulator Range & Conventional-Informal & 1.99 & -14.22 & 18.20 \\
\hline Simulator Range & Conventional-CDL & 7.90 & -3.26 & 19.05 \\
\hline Simulator Range & Focused & & & 22.11 \\
\hline
\end{tabular}

${ }^{*} p<0.05$

The number of failing test scores in each of the testing formats is shown in Table 9. Failing test scores occurred in each group in the simulator road and range tests. Additionally, failing test scores occurred in the informal and CDL-focused groups on the DTCC road test and in the CDLfocused group on the DTCC range test. 
Table 9. Number of Participants that Failed each Test

\begin{tabular}{|l|l|r|r|}
\hline \multicolumn{1}{|c|}{ Measure } & \multicolumn{1}{|c|}{ Training Group } & \multicolumn{1}{c|}{$\begin{array}{c}\text { Number of } \\
\text { Participants Failing } \\
\text { Test }\end{array}$} \\
\hline DTCC Range Test & Conventional & 33 & 0 \\
\hline DTCC Range Test & Simulator & 32 & 0 \\
\hline DTCC Range Test & Informal & 9 & 0 \\
\hline DTCC Range Test & CDL-focused & 33 & 12 \\
\hline DTCC Road Test & Conventional & 33 & 0 \\
\hline DTCC Road Test & Simulator & 32 & 0 \\
\hline DTCC Road Test & Informal & 9 & 6 \\
\hline DTCC Road Test & CDL-focused & 33 & 27 \\
\hline Simulator Range Test & Conventional & 29 & 18 \\
\hline Simulator Range Test & Simulator & 32 & 9 \\
\hline Simulator Range Test & Informal & 29 & 7 \\
\hline Simulator Range Test & CDL-focused & 29 & 26 \\
\hline Simulator Road Test & Conventional & 32 & 5 \\
\hline Simulator Road Test & Simulator & 9 & 2 \\
\hline Simulator Road Test & Informal & 29 & 9 \\
\hline Simulator Road Test & CDL-focused & 28 \\
\hline
\end{tabular}

The CDL-focused group was the only training method where participants failed the DTCC range test. Out of 33 CDL-focused group participants, 12 did not receive passing scores on the DTCC range test. These participants noted they did not have the opportunity to practice some of the maneuvers during their training at their respective schools. Six backing maneuvers (i.e., straight line backing, off-set left, off-set right, alley dock, side-sight parallel park, and conventional parallel park) are in the Delaware DMV range test. However, the Delaware DMV only tests for three of these maneuvers. The two DMV locations where these participants were tested were only configured for the same three backing maneuvers to be examined (i.e., Wilmington DMV is not configured for the alley dock), while other Delaware DMV locations have the ability to test different backing maneuvers. Thus, based on the participants' reports of training, these participants only practiced the backing maneuvers needed to pass the CDL exam at the specific Delaware DMV location.

The informal and CDL-focused groups had 6 and 27 participants fail the DTCC road test, respectively. A wide array of factors contributed to these participants not receiving a passing score. However, proper shifting (i.e., shifts executed into the proper gear without any gear grinding) was a major issue within the informal and CDL-focused groups. Several participants from the CDL-focused group noted that their instructor trained them to shift without the clutch. However, per the Delaware CDL Manual (Delaware DMV, 2005) for testing, the clutch must be used at all times while shifting the vehicle, or it is deemed an error. These participants were instructed to use the clutch for shifting during testing; however, without prior training on doubleclutch shifting a non-synchronized transmission and associated concepts, these participants made numerous errors. Several participants, while in motion, were unable to shift to the next gear and came to a stop in the travel lane. Also, four participants in the CDL-focused group had to be 
stopped by the examiner to prevent a collision (i.e., not accounting for the trailer while turning and hitting a stop sign) during the road test.

Some participants in all four groups did not pass the Simulator range and road tests. One possible explanation for this finding is the lack of depth perception and other sensory cues not associated with a simulated environment. Additionally, those participants not previously trained in the proper concept of shifting a non-synchronized transmission will exhibit the same mistakes in the simulator.

Correlations Pearson product-moment correlation $(r)$ among test types were calculated in each group, based on the test scores in that group. Table 10, Table 11, Table 12, and Table 13 display the correlations between test scores across the conventional, simulator, CDL-focused, and informal training groups, respectively. As shown in Table 10, the conventional training group's DTCC road test was significantly correlated with the DTCC range test $(r=0.54)$ and Simulator road test $(r=0.42)$. The conventional training group's DMV road test was significantly correlated with the DMV range test $(r=0.45)$. No other correlations in the conventional group reached significance.

Table 10. Correlations among Conventional Training Group Driver Test Scores

\begin{tabular}{|l|r|r|r|r|r|r|}
\hline \multicolumn{1}{|c|}{ Measure } & \multicolumn{1}{|c|}{$\begin{array}{c}\text { DTCC } \\
\text { Road } \\
\text { Test }\end{array}$} & $\begin{array}{c}\text { DTCC } \\
\text { Range } \\
\text { Test }\end{array}$ & $\begin{array}{c}\text { Simulator } \\
\text { Road } \\
\text { Test }\end{array}$ & $\begin{array}{c}\text { Simulator } \\
\text { Range } \\
\text { Test }\end{array}$ & $\begin{array}{r}\text { DMV } \\
\text { Road } \\
\text { Test }\end{array}$ & $\begin{array}{c}\text { DMV } \\
\text { Range } \\
\text { Test }\end{array}$ \\
\hline DTCC Road Test & 1 & $0.54^{*}$ & $0.42^{*}$ & -0.11 & 0.14 & 0.00 \\
\hline DTCC Range Test & - & 1 & 0.19 & -0.01 & -0.17 & -0.15 \\
\hline Simulator Road Test & - & - & 1 & 0.09 & 0.07 & 0.18 \\
\hline Simulator Range Test & - & - & - & 1 & -0.09 & -0.13 \\
\hline DMV Road Test & - & - & - & - & 1 & $0.45^{*}$ \\
\hline DMV Range Test & - & - & - & - & - & 1 \\
\hline
\end{tabular}

${ }^{*} p<0.05$

As shown in Table 11, the simulator training group displayed significant correlations between scores on the DTCC road test and the DMV range test $(r=0.38)$, the DTCC range test and the Simulator range test $(r=0.52)$, the Simulator range test and the DMV range test $(r=0.62)$, and the DMV road test and the DMV range test $(r=0.53)$. 
Table 11. Correlations among Simulator Training Group Driver Test Scores

\begin{tabular}{|l|r|r|r|r|r|r|}
\hline Measure & $\begin{array}{c}\text { DTCC } \\
\text { Road } \\
\text { Test }\end{array}$ & \multicolumn{1}{|c|}{$\begin{array}{c}\text { DTCC } \\
\text { Range } \\
\text { Test }\end{array}$} & $\begin{array}{c}\text { Simulator } \\
\text { Road } \\
\text { Test }\end{array}$ & $\begin{array}{c}\text { Simulator } \\
\text { Range } \\
\text { Test }\end{array}$ & $\begin{array}{r}\text { DMV } \\
\text { Road } \\
\text { Test }\end{array}$ & $\begin{array}{c}\text { DMV } \\
\text { Range } \\
\text { Test }\end{array}$ \\
\hline DTCC Road Test & 1 & 0.07 & 0.24 & 0.20 & 0.11 & $0.38^{*}$ \\
\hline DTCC Range Test & - & 1 & -0.05 & $0.52^{*}$ & 0.24 & 0.30 \\
\hline Simulator Road Test & - & - & 1 & 0.13 & 0.07 & 0.04 \\
\hline Simulator Range Test & - & - & - & 1 & 0.33 & $0.62^{*}$ \\
\hline DMV Road Test & - & - & - & - & 1 & $0.53^{*}$ \\
\hline DMV Range Test & - & - & - & - & - & 1 \\
\hline
\end{tabular}

${ }^{*} p<0.05$

As shown in Table 12, the informal training group had a significant, yet moderate, correlation between the DMV road test and the DMV range test $(r=0.67)$. No other correlation reached significance. Note that correlations involving the Simulator road test were not able to be calculated due to all nine participants in the informal training group failing that particular exam (Table 12).

Table 12. Correlations among Informal Group Driver Test Scores

\begin{tabular}{|l|r|r|r|r|r|r|}
\hline \multicolumn{1}{|c|}{ Measure } & $\begin{array}{c}\text { DTCC } \\
\text { Road } \\
\text { Test }\end{array}$ & $\begin{array}{c}\text { DTCC } \\
\text { Range } \\
\text { Test }\end{array}$ & $\begin{array}{c}\text { Simulator } \\
\text { Road Test }\end{array}$ & $\begin{array}{c}\text { Simulator } \\
\text { Range } \\
\text { Test }\end{array}$ & $\begin{array}{r}\text { DMV } \\
\text { Road } \\
\text { Test }\end{array}$ & $\begin{array}{c}\text { DMV } \\
\text { Range } \\
\text { Test }\end{array}$ \\
\hline DTCC Road Test & 1 & 0.30 & - & 0.16 & -0.05 & 0.24 \\
\hline DTCC Range Test & - & 1 & - & 0.36 & 0.00 & 0.55 \\
\hline Simulator Road Test & - & - & - & - & - & - \\
\hline Simulator Range Test & - & - & - & 1 & -0.09 & 0.31 \\
\hline DMV Road Test & - & - & - & - & 1 & $0.67^{*}$ \\
\hline DMV Range Test & - & - & - & - & - & 1 \\
\hline
\end{tabular}

$* p<0.05$

As shown in Table 13, the CDL-focused training group had significant correlations between scores on the DTCC road test and the Simulator road test $(r=0.47)$, as well as between scores on the DMV road test and the DMV range test $(r=0.49)$. 
Table 13. Correlations among CDL-focused Group Driver Test Scores

\begin{tabular}{|l|r|r|r|r|r|r|}
\hline \multicolumn{1}{|c|}{ Measure } & $\begin{array}{c}\text { DTCC } \\
\text { Road } \\
\text { Test }\end{array}$ & $\begin{array}{c}\text { DTCC } \\
\text { Range } \\
\text { Test }\end{array}$ & $\begin{array}{c}\text { Simulator } \\
\text { Road Test }\end{array}$ & $\begin{array}{c}\text { Simulator } \\
\text { Range } \\
\text { Test }\end{array}$ & $\begin{array}{c}\text { DMV } \\
\text { Road } \\
\text { Test }\end{array}$ & $\begin{array}{c}\text { DMV } \\
\text { Range } \\
\text { Test }\end{array}$ \\
\hline DTCC Road Test & 1 & 0.32 & $0.47^{*}$ & -0.17 & -0.14 & -0.04 \\
\hline DTCC Range Test & - & 1 & 0.15 & 0.25 & -0.002 & 0.23 \\
\hline Simulator Road Test & - & - & 1 & -0.06 & -0.11 & -0.02 \\
\hline Simulator Range Test & - & - & - & 1 & 0.03 & 0.11 \\
\hline DMV Road Test & - & - & - & - & 1 & $0.49^{*}$ \\
\hline DMV Range Test & - & - & - & - & - & 1 \\
\hline
\end{tabular}

${ }^{*} p<0.05$

In order to assess if simulator-based testing was equivalent to traditional BTW testing, final scores on the DTCC and Simulator road and range tests were compared. This comparison restricted test scores to those that were administered by the same personnel and independently validated; therefore the DMV tests were excluded from this analysis. The DTCC and Simulator road test scores were compared, for each training group, using paired-samples t-tests. The results indicate that all groups' road scores significantly differed between DTCC and Simulator test modalities ( $p<.05$ in all cases). For the conventional, simulator, and CDL-focused training groups, DTCC road test scores were significantly greater than the Simulator road test scores. Similar results were present for comparison of DTCC and Simulator range test scores using paired-samples t-tests. The DTCC and Simulator range test scores differed significantly for all groups ( $p .0001$ in all cases) and, in all cases, DTCC range test scores were greater than the equivalent simulator range test score. Table 14 provides the results of this analysis.

Table 14. Comparison of DTCC and Simulator road and rage test scores

\begin{tabular}{|l|r|r|r|r|}
\hline \multicolumn{1}{|c|}{ Training Group } & Comparison & \multicolumn{1}{c|}{ Df } & \multicolumn{1}{c|}{$\boldsymbol{t}$} & \multicolumn{1}{c|}{$\boldsymbol{p}$} \\
\hline Conventional & Road Tests & 28 & 5.11 & $<.0001$ \\
\hline Conventional & Range Tests & 28 & 12.84 & $<.0001$ \\
\hline Simulator & Road Tests & 32 & 2.14 & .04 \\
\hline Simulator & Range Tests & 31 & 5.14 & $<.0001$ \\
\hline CDL-focused & Road Tests & 28 & 2.51 & .02 \\
\hline CDL-focused & Range Tests & 28 & 6.64 & $<.0001$ \\
\hline
\end{tabular}

\subsubsection{Discussion}

One of the main reasons to rely on a simulator for testing is to alleviate the costs and requirements that a BTW test might impose. Therefore, it is important the results obtained in a simulator are highly correlated to the ones obtained when the person is tested in a real vehicle, either at a training school or at the DMV, in order to obtain the full benefits of an alternate test modality.

Based on the results obtained in this research effort, the correlations between the different tests were moderate to low. The only training group that had a significant correlation between the one 
of the simulator tests and its DMV test counterpart was in the simulator training group. However, this correlation between the Simulator range test and DMV range test was only moderate.

DMV test scores were not correlated to the simulator test scores in the groups that were not trained on a simulator. Moreover, all nine informal training group participants failed the Simulator road test, and at least one participant in each training group (including the simulator group) failed the Simulator road and range tests. This was not the case on the DTCC and DMV road and range tests. Only participants in the conventional and CDL-focused training groups had a significant, albeit moderate, correlation between the Simulator and DTCC road tests. Additionally, paired-samples $t$-tests indicated that the same participant's performance on DTCC and simulator versions of both road and range tests were statistically different.

These results were consistent and may serve to inform future decisions involving simulator testing. Participants trained in a simulator were able to obtain higher scores than participants who did not receive training in a simulated environment. Thus, it is likely that people who are not trained in a simulator might not be able to pass successfully a Simulator CDL test. These results suggest that there are some aspects unique to the simulated environment that must be experienced and mastered within that environment for testing to reflect skill levels accurately. One potential aspect that might have hindered non-simulator-trained drivers is depth perception. Depending on the simulated environment or the task that was being tested, several landmarks and visual cues obtained through depth perception were not present in the simulated environment, thereby making tasks such as backing more difficult in the simulated environment.

\subsubsection{Research Question 2b: What is the correlation between scores from the DTCC road} and range tests and simulator road and range tests?

\subsubsection{Method and Results}

During each test (road or range), participants completed a number of maneuvers (scoring aspects). In each maneuver, participants were scored on a number of factors. For example, participants performing the straight line backing maneuver in range testing were scored on the number of pull-ups, encroachments, times they left the vehicle to look at their position, and their final position. Participants accumulate points as errors were made in any of the scored aspects of the test (e.g., number of pull-ups made during straight line backing); perfect performance on any one aspect of a test was represented by a score of 0 .

In order to perform meaningful comparisons between Simulator and DTCC testing, count data were summed across all scored aspects in each test (e.g., a total score for "straight line backing"). From these, a Pearson product-moment correlation was performed between the DTCC and Simulator road tests, as well as between the DTCC and Simulator range tests. Note that if a participant failed the test, the test would immediately stop and no further maneuvers were scored. Thus, only those scoring aspects with a complete pair of scores (Simulator and DTCC) were included in the present analysis.

Note that the number of participants presented in the tables and figures below reflect the number included in that specific analysis and could be lower than the actual total number of participants in that training group. For example, out of the 33 conventional training group participants' 
results, 29 were available for the Simulator road test as 4 participants experienced simulator sickness and were not able to perform the test. Only participants with a given score aspect in both the Simulator and DTCC tests were used in the analyses presented next.

\subsubsection{Road Tests}

Participants were scored on 13 aspects of driving performance during the road test:

General driving (e.g., shifting, gear grinding, steering, and braking).

Left lane changes.

Right lane changes.

Left turns.

Right turns.

Road side pull-offs.

Railroad crossings.

Serious errors (e.g., traffic violations, dangerous actions, putting the vehicle on sidewalks or curbs).

Road sign compliance.

Stopping/braking performance.

Intersection performance.

Left curves.

Right curves.

Pearson product-moment correlations and descriptive statistics for both DTCC and Simulator road test scores across groups are provided in Table 15.

Correlations between the scored aspects of the DTCC and Simulator road tests were calculated. Significant correlations were observed in the conventional training group on the general driving and left turn categories ( $r=0.49$ and 0.43 , respectively). Significant correlations were observed in the simulator training group on general driving and right turn categories $(r=0.54$ and 0.53 , respectively). In some cases no participants in a group passed the section or all participants in a group passed a section; thus, some correlations could not be calculated. 


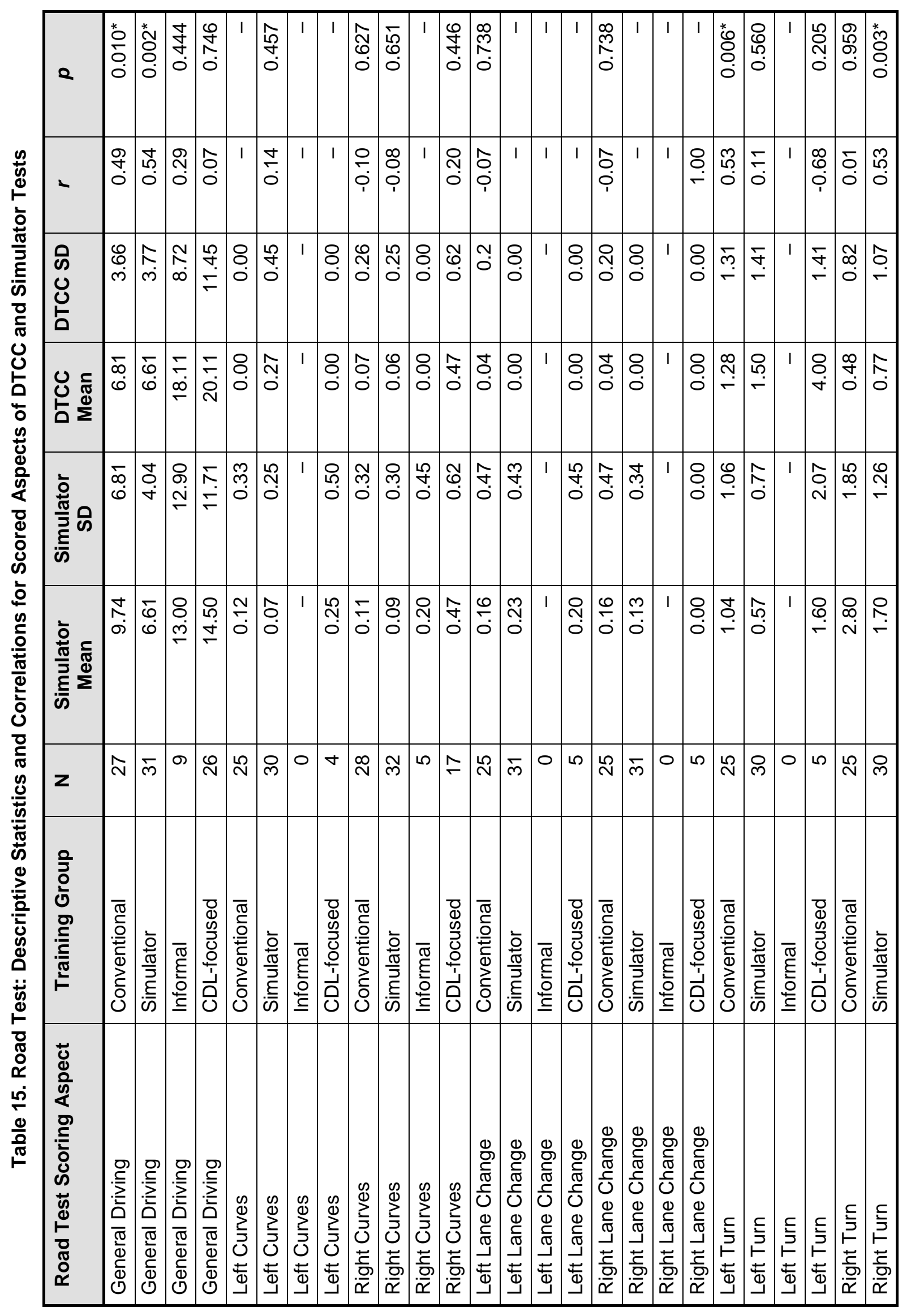




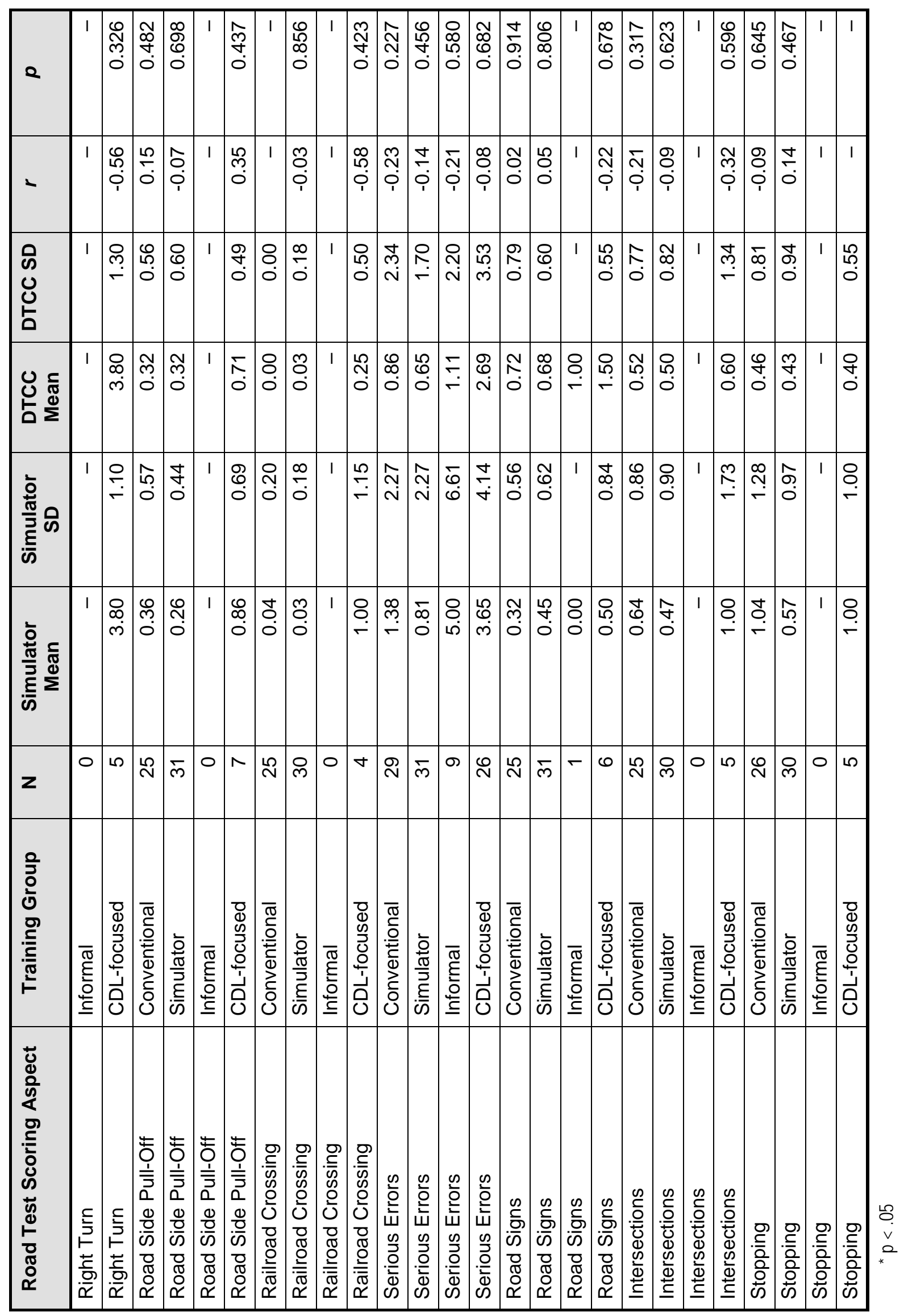


Using a Wilcoxon signed rank test performance on the DTCC and Simulator road test scoring aspects (shown in the Road Test Scoring Aspects column of Table 15, above) were individually compared for the conventional and simulator training groups. This comparison illustrates if a simulator-testing environment is appropriate for drivers trained in a BTW environment and if simulator-trained drivers can be tested in a simulator.

For the conventional training group, results indicate that significant differences were present for general driving $(t(26)=-2.55, p=0.02)$, road sign compliance $(t(24)=2.09, p=0.048)$, and right turns $(t(24)=-5.76, p<0.0001)$. The conventional training group had more general driving demerit points, more right turn error points, and fewer road sign error points, when testing in the Simulator versus DTCC testing. This finding suggests that the tests were not equivalent, and drivers trained in a BTW setting will not have equivalent performance in simulator and BTW tests of road driving skills.

For the simulator training group, significant differences were present for left curves $(t(29)=$ 2.26, $p=0.03)$, left turns $(t(29)=3.34, p=0.002)$, right turns $(t(29)=-4.47, p=0.0001)$, left lane changes $(t(30)=-2.96, p=0.006)$, and right lane changes $(t(30)=-2.11, p=0.04)$. The simulator training group had fewer left curve and left turn errors, and greater right turn, left lane change, and right lane change errors, when testing in the Simulator versus DTCC testing. This finding suggests that the tests were not equivalent, and drivers primarily trained in a simulator will not have equivalent performance in simulator and BTW tests of road driving skills.

Even if the evaluators are well trained and possess a Class A CDL and extensive years of experience, some components of the testing procedure are still subjective. Therefore, several objective components were identified and post-processed after the data were collected. Taking advantage of the "instant replay" capabilities of the DAS, data reduction was performed on participant road tests in DTCC and Simulator modalities across the four groups in the following three areas:

Lane Departure (LD), defined as events where the truck or trailer crossed the boundaries of the intended travel lane due to improper driver control or driver inattention. These data were obtained from the side-mounted cameras (directed towards the lateral edges of the truck and trailer) and the DAS's onboard lane detection system.

Longitudinal Acceleration (LA), defined as events where the vehicle was forced to accelerate or brake suddenly in order to avoid a collision or other hazardous event. The criteria for this event were vehicle speed at or above $5 \mathrm{mi} / \mathrm{h}(8 \mathrm{~km} / \mathrm{h})$ and acceleration above an absolute value of $0.20 \mathrm{~g}$. These data was obtained from the onboard DAS accelerometer.

Time to Collision (TTC), defined as events where the vehicle had a TTC with a forward object of less than or equal to $2.0 \mathrm{~s}$. These data were obtained from the radar unit mounted on the front bumper of the truck.

Based on these criteria, the data were processed to identify instances of these events. These potential events were validated by trained human reviewers who compared the identified event with the criteria and identified the event as valid or invalid. The results in this report present only those events verified as valid by human reviewers. 
Based on the frequency of each event and the duration (in minutes) of each test, rates were calculated for LDs, LAs, and TTCs. Table 16 shows the rate per minute of each event for each test mode across all groups. The majority of events, regardless of group membership or test modality, were LDs. A much smaller number of valid LA or TTC events were present, and some groups had no valid LA or TTC events within a test mode. Thus, the accompanying rates for those event types are accordingly low. 


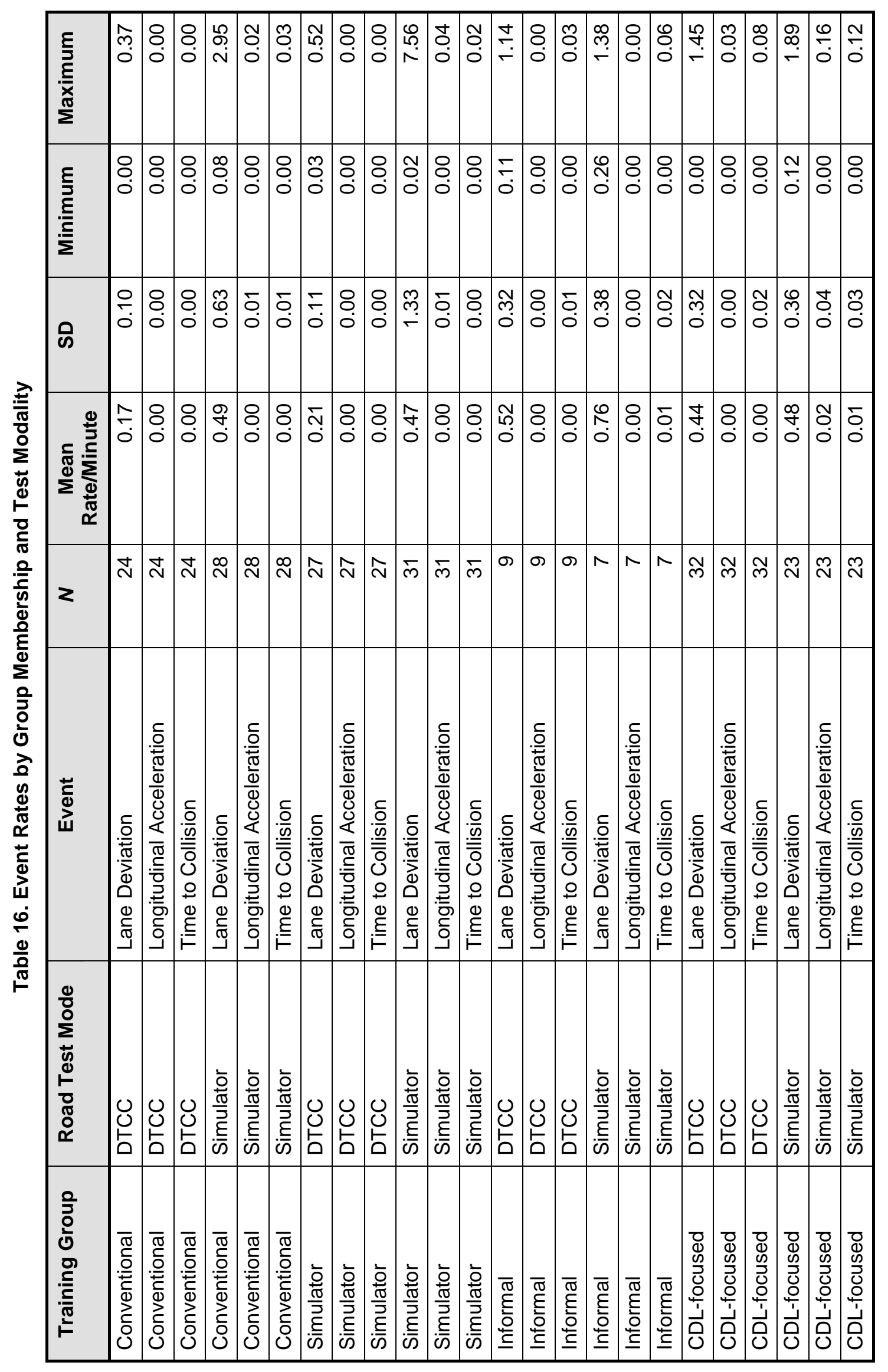


As the only event type with reliable appearances in all groups and test modes, further analysis was conducted on LD rates. A separate data set was created containing each participant's DTCC and Simulator validated LD rates per minute. Participants with missing data, in either DTCC or Simulator tests, were excluded from the analysis. Table 17 shows the resultant data set with validated LD rates from 76 participants.

Table 17. Lane Deviations, by Group Membership and Test Mode

\begin{tabular}{|l|l|r|c|r|r|r|}
\hline Training Group & Road Test Mode & \multicolumn{1}{|c|}{$\boldsymbol{N}$} & Mean Rate/Minute & \multicolumn{1}{c|}{ SD } & \multicolumn{1}{c|}{ Min } & \multicolumn{1}{c|}{ Max } \\
\hline Conventional & DTCC & 20 & 0.17 & 0.10 & 0.00 & 0.37 \\
\hline Conventional & Simulator & 20 & 0.45 & 0.47 & 0.10 & 2.06 \\
\hline Simulator & DTCC & 27 & 0.21 & 0.11 & 0.03 & 0.52 \\
\hline Simulator & Simulator & 27 & 0.51 & 1.42 & 0.02 & 7.56 \\
\hline Informal & DTCC & 7 & 0.49 & 0.20 & 0.15 & 0.81 \\
\hline Informal & Simulator & 7 & 0.76 & 0.38 & 0.26 & 1.38 \\
\hline CDL-focused & DTCC & 22 & 0.42 & 0.28 & 0.00 & 1.33 \\
\hline CDL-focused & Simulator & 22 & 0.47 & 0.37 & 0.12 & 1.89 \\
\hline
\end{tabular}

Difference scores were calculated between LD rates (Figure 13) in the DTCC and Simulator road tests; a Kruskal-Wallis chi-square test was conducted to examine for differences in LD rates between test modality based on group membership. The result indicated a significant group difference in the difference scores, $\chi^{2}(3, N=76)=8.06, p=0.04$. Follow-up testing via Wilcoxon signed rank tests indicated that the only significant group difference was in the conventional training group, $(t(19)=-2.80, p=0.01)$. Thus, participants in the conventional training group had a greater rate of LDs in the Simulated road test as compared to the DTCC road test.

However, these findings of a difference in LD rates between test modes must be considered with each group's likelihood of passing or failing the exam. Conventional training group participants were more likely to fail the Simulated road test as compared to the DTCC road test $(t(19)=3.62$, $p=.002)$. In comparison, the simulator training group did not have a difference in passing likelihood between tests $(p>.05)$, while all informal $(M=50.00, S D=0.00)$ participants and all but one CDL-focused $(M=51.07, S D=5.76)$ participant failed the Simulator tests. Lane departures, presented by group and test modality, are depicted in Figure 13. 


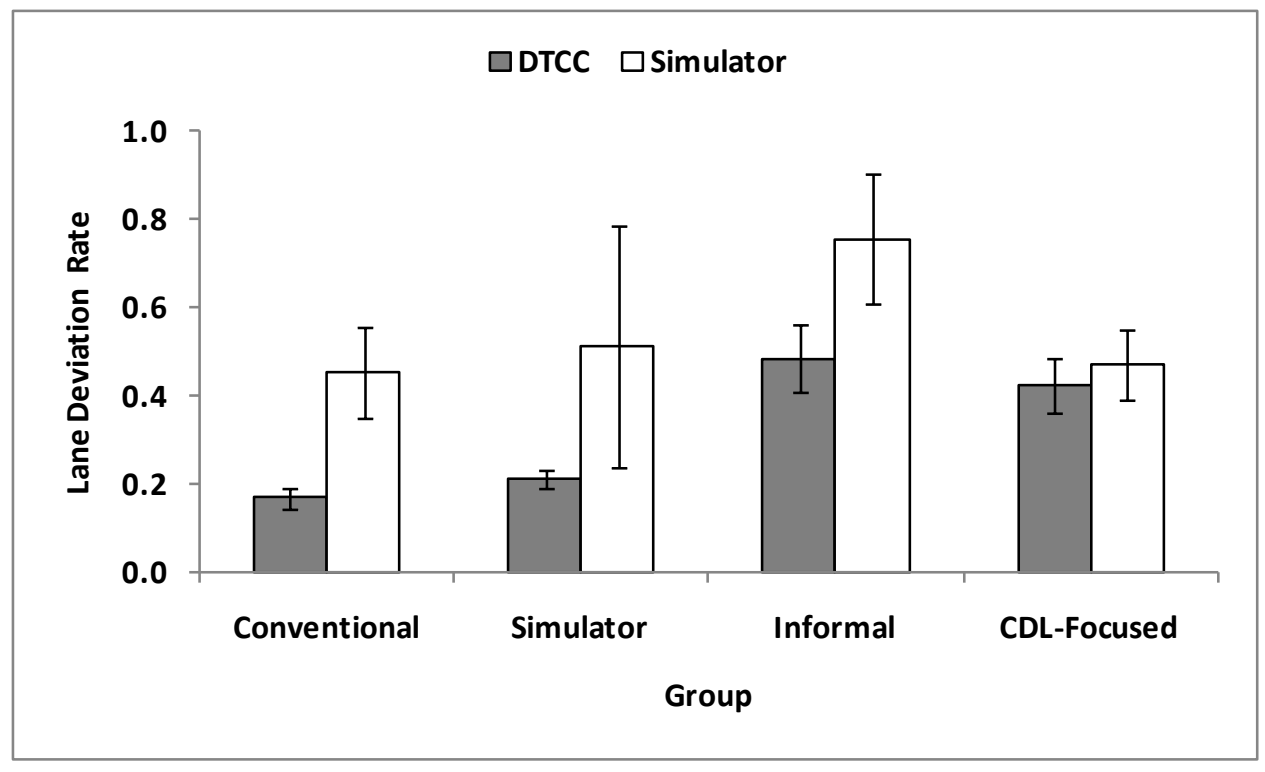

Figure 13. Chart. Lane departures by group membership and test modality.

Note: only participants with DTCC and Simulator test data are included. Error bars represent standard error.

Further analysis was conducted comparing the conventional and simulator training groups on the LD rate in DTCC road testing. This is an important analysis, as it is reflective of whether or not drivers trained in a simulator will have equivalent lateral control skills to drivers who receive the same curriculum delivered in BTW training. The DTCC road test LD rates did not differ between conventional and simulator (Kruskal-Wallis $\left.\chi^{2}(1, N=51)=1.12, p=0.29\right)$. This demonstrates that conventional and simulator training group drivers (who only differed in training mode, Simulator versus BTW) have equivalent lateral control skills as reflected in LD rates.

The four groups were also clustered by whether or not their training was PTDI-certified. This created two comparison groups: one consisting of participants with PTDI certified training (conventional and simulator training groups, both of which included the PTDI certification's minimum 44 hours of instructor-present driving time) and one of participants who trained in nonPTDI certified manners (informal and CDL-focused training groups, neither of which have a minimum number of instructor-present driving hours). A Kruskal-Wallis chi-square test indicated a significant difference in LD rate between clustered groups $\left(\chi^{2}(1, N=92)=26.23\right.$, $p<0.0001)$. The PTDI certified trained participants had a significantly lower mean rate of DTCC road test LDs $(M=0.19, S D=0.11, n=51)$ as compared to the non-PTDI-certified trained participants $(M=0.46, S D=0.31, n=41)$. This indicates that participants who trained in a PTDI certified program had greater lateral control skills, as reflected in LD rates, when compared to participants not trained in PTDI-certified programs.

\subsubsection{Range Tests}

Participants were scored on six aspects of driving performance during the range tests: straight line backing, off-set left backing, off-set right backing, alley docking, side-sight parallel parking, and conventional parallel parking. Correlations between, and descriptive statistics for, DTCC and Simulator range test scores across groups are provided in Table 18. 
Table 18. Range Test: Descriptive Statistics and Correlations for Scored Aspects of DTCC and Simulator Tests

\begin{tabular}{|l|l|r|r|r|r|r|r|r|}
\hline $\begin{array}{c}\text { Range Test Scoring } \\
\text { Aspects }\end{array}$ & \multicolumn{1}{|c|}{$\begin{array}{c}\text { Training } \\
\text { Group }\end{array}$} & $\boldsymbol{N}$ & $\begin{array}{c}\text { Simulator } \\
\text { Mean }\end{array}$ & $\begin{array}{c}\text { Simulator } \\
\text { SD }\end{array}$ & $\begin{array}{c}\text { DTCC } \\
\text { Mean }\end{array}$ & $\begin{array}{c}\text { DTCC } \\
\text { SD }\end{array}$ & $\boldsymbol{r}$ & $\boldsymbol{p}$ \\
\hline Straight Line Backing & Conventional & 29 & 2.31 & 6.05 & 0.14 & 0.52 & 0.21 & 0.263 \\
\hline Straight Line Backing & Simulator & 31 & 0.71 & 2.05 & 0.00 & 0.00 & - & - \\
\hline Straight Line Backing & Informal & 9 & 1.89 & 4.96 & 0.22 & 0.67 & -0.14 & 0.714 \\
\hline Straight Line Backing & CDL-focused & 26 & 3.00 & 5.95 & 0.69 & 1.57 & 0.03 & 0.901 \\
\hline Off-Set Left & Conventional & 27 & 3.59 & 5.02 & 0.00 & 0.00 & - & - \\
\hline Off-Set Left & Simulator & 31 & 2.06 & 4.31 & 0.32 & 1.08 & 0.33 & 0.074 \\
\hline Off-Set Left & Informal & 9 & 5.67 & 5.55 & 0.00 & 0.00 & - & - \\
\hline Off-Set Left & CDL-focused & 19 & 5.11 & 4.95 & 0.58 & 1.30 & 0.33 & 0.162 \\
\hline Off-Set Right & Conventional & 25 & 3.32 & 3.60 & 0.00 & 0.00 & - & - \\
\hline Off-Set Right & Simulator & 29 & 0.93 & 1.19 & 0.10 & 0.41 & 0.38 & $0.042^{*}$ \\
\hline Off-Set Right & Informal & 9 & 4.22 & 3.63 & 0.11 & 0.33 & 0.39 & 0.299 \\
\hline Off-Set Right & CDL-focused & 18 & 3.61 & 4.16 & 0.11 & 0.47 & 0.32 & 0.191 \\
\hline Alley Dock & Conventional & 19 & 7.37 & 5.71 & 0.79 & 1.72 & -0.01 & 0.954 \\
\hline Alley Dock & Simulator & 27 & 3.74 & 4.55 & 0.81 & 1.55 & 0.58 & $0.001^{*}$ \\
\hline Alley Dock & Informal & 4 & 5.50 & 4.43 & 0.50 & 1.00 & -0.83 & 0.173 \\
\hline Alley Dock & CDL-focused & 4 & 6.75 & 4.50 & 4.50 & 5.45 & 0.96 & $0.041^{*}$ \\
\hline Side-sight Parallel & Conventional & 15 & 5.33 & 4.27 & 0.13 & 0.52 & 0.42 & 0.121 \\
\hline Side-sight Parallel & Simulator & 25 & 2.20 & 2.99 & 0.92 & 1.58 & 0.44 & $0.029^{*}$ \\
\hline Side-sight Parallel & Informal & 4 & 5.75 & 4.50 & 1.25 & 0.96 & -0.06 & 0.942 \\
\hline Side-sight Parallel & CDL-focused & 8 & 7.25 & 4.46 & 4.13 & 4.52 & 0.19 & 0.653 \\
\hline Conventional Parallel & Conventional & 14 & 5.86 & 5.01 & 1.07 & 3.20 & -0.12 & 0.672 \\
\hline Conventional Parallel & Simulator & 24 & 2.33 & 3.84 & 0.88 & 1.99 & 0.42 & $0.043^{*}$ \\
\hline Conventional Parallel & Informal & 2 & 1.00 & 1.41 & 1.00 & 1.41 & 1.00 & - \\
\hline Conventional Parallel & CDL-focused & 7 & 5.71 & 3.45 & 3.71 & 6.02 & 0.61 & 0.143 \\
\hline
\end{tabular}

$$
{ }^{*} p<.05
$$

Significant correlations were observed for the simulator training group on off-set right backing, alley docking, side-sight parallel parking, and conventional parallel parking $(r=0.38,0.58,0.44$, and 0.42, respectively). The CDL-focused Training Group displayed a significant correlation between DTCC and Simulator range test scores on the alley docking maneuver $(r=0.96)$. As above, in some cases correlations were unable to be calculated (due to either no participants in a group passing the section or all participants passing a section with no points taken off their scores).

Using a Wilcoxon signed rank tests, performance on the DTCC and Simulator range test scoring aspects (shown in Table 18) were individually compared for the Conventional and simulator training groups. This comparison illustrates if a simulator testing environment is appropriate for drivers trained in a BTW environment and if simulator-trained drivers may test in a simulator. 
For the conventional training group, results indicate that significant differences were present for off-set right $(t(24)=-4.61, p=.0001)$, off-set left $(t(26)=-3.72, p=.001)$, alley dock $(t(18)=$ 4.79, $p=.0001)$, side-sight parallel $(t(14)=-5.12, p=.0002)$, and conventional parallel $(t(13)=$ $2.86, p=.01)$ maneuvers. For each significant difference in scoring aspects, the conventional training group had more error points when testing in the simulator versus DTCC range testing. This finding suggests that the DTCC and simulator tests were not equivalent, and drivers trained in a BTW manner will not have equivalent performance in simulator and BTW tests of range skills.

For the simulator training group, significant differences were present for off-set right $(t(28)=$ $4.04, p=.0004)$, off-set left $(t(30)=-2.37, p=.02)$, alley dock $(t(26)=-3.94, p=.0006)$, and side-sight parallel $(t(24)=-2.37, p=.03)$ maneuvers. Similar to the comparison of conventional training group range scoring aspects, in each significant comparison the simulator training group had more error points when testing in the Simulator versus DTCC testing. This finding suggests that the DTCC and simulator tests were not equivalent, and drivers trained primarily in a simulator will not have equivalent performance in simulator and BTW tests of range skills.

\subsubsection{Discussion}

There were 13 scoring areas compared in the road tests and 6 in the range tests. Not all 13 road test scoring areas had a significant correlation between the Simulator and DTCC tests. However, scoring aspects related to general driving and turns had significant correlations in the conventional and simulator groups. None of the road test scoring aspects in the Informal and CDL-focused groups' participants were correlated with the Simulator and DTCC tests. This suggests that in the road test, the results obtained in a simulator will not always resemble the results obtained BTW. Some participants might be able to demonstrate their skills in a similar manner in both environments (i.e., simulated and BTW), but the results obtained in one might not be related to the other (at least not in a linear relationship). Based on this set of results, drivers trained in a PTDI-certified program were the only ones able to demonstrate their general driving skills in both a simulator and BTW (e.g., shifting, clutch use, gear selection, braking, steering, lane usage, and traffic checks).

The results from the range tests were not parallel to the results obtained in the road tests. In the range test, the simulator group had significant correlated results between the DTCC and simulator environments in most of the scoring aspects. Participants in the CDL-focused group were able to demonstrate their skills in a similar manner in both DTCC and simulator environments during the alley dock. However, this result was based on only four comparisons. Straight line backing was the only scenario that did not present similar results in both testing environments in any of the groups. It should be noted that the simulator group had relatively high performance in both the DTCC $(M=0, S D=0.00$ demerit points $)$ and Simulator $(M=0.71, S D=$ 2.05 demerit points) straight line backing tasks. The main reason for this might be that practice and experience with simulated tasks are needed in order to habituate to and learn the simulator's depth perception cues.

Analysis of the data recorded in DTCC and Simulated read tests during LD, LA, and TTC events revealed both similarities and differences in the two versions of the road test. The number of LA and TTC events in all test modalities was very low. This was not an unanticipated outcome as participants who trained for these tests (even if in an informal or temporally-compressed manner) 
were actively observed by an instructor. However, control over the articulated vehicle presents a much more challenging task, and this was revealed in the high rate of LD events observed in all test modes, across all groups. Further analysis of LD rates indicated there was a difference in the rate of LDs in DTCC and Simulated road tests by group. Participants in the conventional training group had a greater rate of LDs in the simulated road test as compared to the DTCC road test. No other group displayed any reliable differences in LDs based on test mode. As with the range tests, the lack of rich perceptual depth cues in the simulated environment may be one reason for this. Participants in the conventional training group were also more likely to fail the Simulated road test as compared to the DTCC road test; therefore, this difference may be part of a larger trend in conventional training group participants having higher performance in the DTCC as compared to Simulated tests. Note that both informal (all participants failed the simulated road test) and CDL-focused (only one participant passed the simulated road test) training groups had low performance on the Simulator road test. Thus, this pattern may not have manifested as a greater number of LDs due to the tests stopping earlier for almost all the participants in these groups.

When the rate of DTCC road test LDs was compared between conventional and simulator training groups, no significant differences were found. This is an important finding as it demonstrates that participants who receive most of their entry-level CMV driver training in a simulator have equivalent BTW test performance to participants who only trained in real trucks. When the groups were clustered based on whether or not they had attended a PTDI-certified entry-level CMV driver training program, interesting results were found. Participants who received PTDI-certified training had a significantly lower rate of LD as compared to those participants who did not have PTDI-certified training. While this is only one method of assessing the road driving skill levels of participants, lateral control is an important aspect of vehicle control. The difference may be a reflection of the increased instructor presence during driving training that participants in a PTDI-certified program experience. Additionally, the fact that simulator trained drivers are equivalent to conventionally trained drivers is important for the use of simulators as CMV driver training platforms. Likewise, the finding that LD rate is lower for drivers with PTDI-certified training holds important implications for future research into training standards and minimum training levels.

\subsubsection{Research Question 2c: Which road and range test scores (DTCC or Simulated) are more predictive of subsequent DMV road and range tests?}

This research question sought to determine the relationship between DTCC and Simulated road and range tests with the DMV road and range tests. The hypothesis for this research question was "there will be a strong statistical correlation between drivers' scores on both tests (Simulated and DTCC road/range test) and the DMV road/range test scores.”

\subsubsection{Method and Results}

Correlations were calculated between DTCC, Simulated, and DMV road and range tests in the course of the Research Question 2a analysis. As shown in the results of Research Question 2a, there were no statistically significant correlations between DTCC and DMV versions of road tests for any group. The Simulator and DMV versions of the range tests were significantly correlated in the simulator training group $(r=.62, p<.0005)$. No other group had a significant correlation between Simulator and DMV range tests. 


\subsubsection{Discussion}

As mentioned previously only the simulator training group, showed a significant correlation between the simulator tests and the DMV test counterpart. However, the correlation between Simulator and DMV range tests for the simulator training group was not a very high $(r=.62)$. DMV scores were not correlated to the Simulator test scores for the groups that were not trained in a simulator. Moreover, all nine informal training participants failed the Simulator road test and at least one participant in each training group (including the simulator group) failed the Simulator road and range test.

As indicated above, participants trained in a simulator were able to obtain higher road and range scores than participants not trained in a simulated environment. Moreover, some participants who were not trained in a simulator had difficulty passing the simulator tests. These findings suggest that some aspects of the simulator that relate to test performance must be practiced within that specific environment in order for a driver to successfully test within the simulated environment. One such potential aspect that might have hindered non-simulator-trained drivers is depth perception. Depending on the simulated environment or the task that is being tested, several landmarks and cues obtained through depth perception are not present in a simulated environment, making tasks such as backing more difficult in a simulated environment.

\subsubsection{Research Question 2d: What features of the simulator will be most and least effective for participants primarily trained on the simulator?}

This research question sought to identify which features of the simulator the participants thought were most and least effective for entry-level training. These data were collected from participants through a questionnaire given at the end of entry-level driver training. Participants were provided with a list of 10 aspects of the simulator (e.g., safety, overhead views, repeatability, standardization, realism, enrichment/the ability of the simulator to be used for defensive driving training, basic control, vehicle handling, safe operations, and backing skills; see Table 4 for the operational definition of these aspects) and provided their top three aspects that were most and least effective (for a total of six rankings). Only the simulator training group participants $(n=30)$ completed this specific section of the questionnaire. Two participants chose not to respond to this questionnaire.

Responses were weighted based on ranking (1st ranking = 10; 2nd ranking = 9, 3rd ranking = 8). This allowed for an overall score to be assigned to each aspect. Overall, the most effective aspect was basic control, while the second most effective aspect was a tie between overhead views and repeatability. Vehicle handling was the third most effective aspect. The least effective aspect of the simulator was realism, followed by backing skills and enrichment, respectively. Results for most effective and least effective aspects of the simulator are provided in Figure 14. 


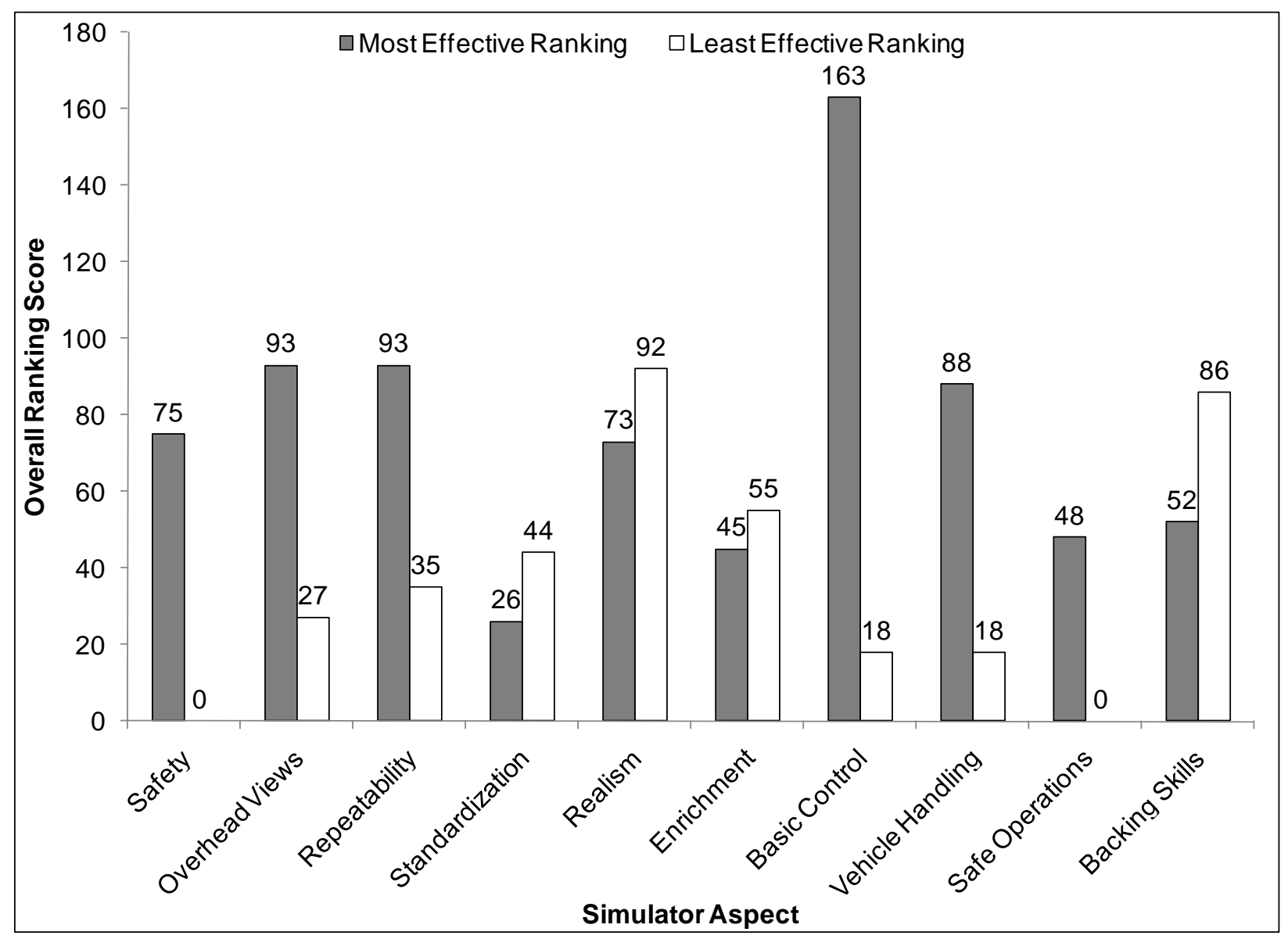

Figure 14. Chart. Overall ranking scores for simulator aspects.

\subsubsection{Discussion}

The results obtained in this subjective assessment supplement the results obtained in the previous analyses. Similar to what was inferred from the correlation analysis, the simulator allows drivers trained in this driving environment to practice the basic controls needed on a vehicle (referred to in the road test as general driving skills). Previous research by Robin et al., (2005b) suggests that repeatability and the ability to obtain overhead views and other perspectives of where the vehicle is positioned are very important aspects of a simulator. These findings confirm the importance of these two aspects, and are two of the main reasons why a simulator should be considered for training purposes. While the realism of simulators has improved dramatically over the years, a simulated environment is still not capable of portraying or potentially engaging a driver the same way a real-world scenario might be able to. However, not all scenarios could be repeated safely and economically in a real-world environment.

\subsection{TOPIC \#3: LONGITUDINAL EFFECTS OF TRAINING METHOD ON PARTICIPANT EVALUATION OF TRAINING}

As part of this topic five research questions were addressed: 
Research Question 3a: Does training method affect the self-ratings of a driver's current performance?

Research Question 3b: How does training method affect participant retrospective self-ratings of the training method received in terms of teaching vehicle control, maneuvering skills, and safe operations in comparison to their peers?

Research Question 3c: Does training method affect trainee evaluation of training at the end of training and 4 months after being employed as a CDL driver?

Research Question 3d: Would participants trained primarily on the simulator deem the simulator skill tests as a valid and fair assessment of their driving skills at the time they completed their training programs?

Research Question 3e: Would participants recommend their method of training to other prospective commercial driver trainees?

The method, analysis, measures of interest, results, and discussion related to these five research questions are presented next.

\subsubsection{Method}

This set of research questions focused on the subjective assessment of the driver, training method, and training satisfaction. Questionnaire data were obtained from participants after 4-5 months after being employed (see appendix $\mathrm{G}$ for the follow-up self assessment). In addition, Research Question 3c involved a comparison between participants' opinions on training received immediately at the completion of training and at the 4 month-5-month period after being employed. This information was gathered from the End of Testing Participant Assessment (see appendix L). Due to the economic downturn leading to one of the greatest number of trucking job losses recorded-more than 100,000 jobs lost between 2008 and 2009 (Howard, Zukerman, Strah, \& McNally, 2009) — a number of participants had difficulty in finding employment as a CMV driver. Only a small number of study participants were able to secure employment that required use of their Class A CDL. Compounding these participants' difficulty in finding employment was the decision by many trucking companies to recruit only experienced drivers, making it more difficult for newly licensed drivers to find employment (Bureau of Labor Statistics, 2009). These issues were unforeseen at the time this study was designed. As such, a more in-depth examination of results is not possible due to the small number of participants finding employment as CMV drivers. Therefore, descriptive statistics are presented for these research questions.

\subsubsection{Research Question 3a: Does training method affect the self-ratings of drivers’ current performance?}

These questions asked drivers to compare themselves to other Class A CDL drivers with equivalent driving experience (approximately 4-5 months on the job) and rate their current vehicle control and maneuvering skills (e.g., proper shifting, smooth acceleration and braking) and safe operations skills (e.g., defensive driving, speed maintenance, space management). Participants provided ratings on a scale of 1-5, with 1 indicating that they were "one of the worst" and 5 indicating that they were "one of the best." 
As shown in Table 19, participants had a slightly above average mean self-assessment in their control and safety skills. This was present regardless of group membership. However, an examination of the range of responses indicated that drivers in the simulator group may have been more willing to be self-critical compared to the other groups. Additionally, the three drivers in the CDL-focused training group indicated they were above average in comparison to other Class A drivers with their experience.

Table 19. Descriptive Statistics for Participants' Current Self-rating of Control and Safety Skills

\begin{tabular}{|l|l|r|r|r|r|r|}
\hline Training Group & \multicolumn{1}{|c|}{ Measure } & \multicolumn{1}{c|}{$\boldsymbol{N}$} & \multicolumn{1}{c|}{ Mean } & \multicolumn{1}{c|}{ SD } & Minimum & Maximum \\
\hline Conventional & Control Skills & 6 & 3.67 & 0.52 & 3 & 4 \\
\hline Conventional & Safety Skills & 6 & 4.00 & 0.63 & 3 & 5 \\
\hline Simulator & Control Skills & 15 & 3.87 & 0.99 & 2 & 5 \\
\hline Simulator & Safety Skills & 15 & 4.13 & 0.83 & 2 & 5 \\
\hline Informal & Control Skills & 3 & 3.67 & 0.58 & 3 & 4 \\
\hline Informal & Safety Skills & 3 & 4.00 & 0.00 & 4 & 4 \\
\hline CDL-focused & Control Skills & 3 & 4.00 & 0.00 & 4 & 4 \\
\hline CDL-focused & Safety Skills & 3 & 4.00 & 0.00 & 4 & 4 \\
\hline
\end{tabular}

5.4.3 Research Question 3b: How does training method affect participant retrospective self-ratings of the training method received in ter ms of teaching vehicle control, maneuvering skills, and safe operations in comparison to their peers?

This question asked drivers to compare themselves to other Class A CDL drivers with equivalent driving experience (approximately 4-5 months on the job) and rate the training they received with respect to vehicle control and maneuvering skills (e.g., proper shifting, smooth acceleration \& braking) and safe operations skills (e.g., defensive driving, speed maintenance, space management). Participants provided ratings on a scale of $1-5$, with 1 indicating that the training they received was "very poor" and 5 indicating that the training they received was "very good."

Results (shown in Table 20) indicated that participants rated their respective method of training as between average and above average. The range of responses in most groups was between 3 and 4, although a greater range of responses was observed in the simulator training group (range: 2-5) and CDL-focused training group (range: 3-5).

Table 20. Descriptive Statistics for Participants' Retrospective Rating of Training

\begin{tabular}{|l|l|r|r|r|r|r|}
\hline $\begin{array}{c}\text { Training } \\
\text { Group }\end{array}$ & \multicolumn{1}{|c|}{ Teaching Aspect } & \multicolumn{1}{c|}{$\boldsymbol{n}$} & \multicolumn{1}{c|}{ Mean } & \multicolumn{1}{c|}{ SD } & Minimum & Maximum \\
\hline Conventional & Control Skills & 6 & 3.50 & 0.55 & 3 & 4 \\
\hline Conventional & Safety Skills & 6 & 3.67 & 0.52 & 3 & 4 \\
\hline Simulator & Control Skills & 15 & 3.60 & 0.91 & 2 & 5 \\
\hline Simulator & Safety Skills & 15 & 3.80 & 0.94 & 2 & 5 \\
\hline Informal & Control Skills & 3 & 3.33 & 0.58 & 3 & 4 \\
\hline Informal & Safety Skills & 3 & 3.00 & 0.00 & 3 & 3 \\
\hline CDL-focused & Control Skills & 3 & 4.00 & 1.00 & 3 & 5 \\
\hline CDL-focused & Safety Skills & 3 & 3.67 & 0.58 & 3 & 4 \\
\hline
\end{tabular}


5.4.4 Research Question 3c: Does training method affect trainee evaluation of training at the end of training and 4 months after being employed as a CDL driver?

Participant ratings regarding the entry-level training they received on vehicle control and maneuvering skills (e.g., proper shifting, smooth acceleration \& braking) and safe operations skills (e.g., defensive driving, speed maintenance, space management) at 4-5 months after being employed were compared to the same ratings given at the end of entry-level training .

Participants provided ratings on a scale of 1-5, with 1 indicating that the training they received was "very poor" and 5 indicating that the training they received was "very good." Note that two participants in the simulator and conventional training groups chose not to respond. Due to imbalances in the number of participants per group, only descriptive statistics are presented. Participant ratings of training at the end of entry level training and at the 4-5 month follow-up are presented in Table 21.

Table 21. Rating of Training at End of Training and 4-months After Being Hired

\begin{tabular}{|l|l|r|r|r|r|r|}
\hline Training Group & \multicolumn{1}{c|}{ Measure } & \multicolumn{1}{c|}{$\boldsymbol{n}$} & \multicolumn{1}{c|}{ Mean } & \multicolumn{1}{c|}{ SD } & Minimum & Maximum \\
\hline Conventional & End of Training Control Skills & 31 & 3.90 & 0.65 & 2 & 5 \\
\hline Conventional & End of Training Safety Skills & 31 & 4.00 & 0.68 & 2 & 5 \\
\hline Conventional & 4 Months Post Control Skills & 6 & 4.67 & 0.52 & 4 & 5 \\
\hline Conventional & 4 Months Post Safety Skills & 6 & 4.67 & 0.52 & 4 & 5 \\
\hline Simulator & End of Training Control Skills & 28 & 4.64 & 0.73 & 2 & 5 \\
\hline Simulator & End of Training Safety Skills & 28 & 4.68 & 0.61 & 3 & 5 \\
\hline Simulator & 4 Months Post Control Skills & 15 & 4.67 & 0.82 & 2 & 5 \\
\hline Simulator & 4 Months Post Safety Skills & 15 & 4.87 & 0.35 & 4 & 5 \\
\hline Informal & End of Training Control Skills & 9 & 3.78 & 0.67 & 3 & 5 \\
\hline Informal & End of Training Safety Skills & 9 & 3.89 & 0.78 & 3 & 5 \\
\hline Informal & 4 Months Post Control Skills & 3 & 4.33 & 0.58 & 4 & 5 \\
\hline Informal & 4 Months Post Safety Skills & 3 & 4.33 & 0.58 & 4 & 5 \\
\hline CDL-focused & End of Training Control Skills & 33 & 4.03 & 0.88 & 1 & 5 \\
\hline CDL-focused & End of Training Safety Skills & 33 & 4.30 & 0.73 & 2 & 5 \\
\hline CDL-focused & 4 Months Post Control Skills & 3 & 4.00 & 1.00 & 3 & 5 \\
\hline CDL-focused & 4 Months Post Safety Skills & 3 & 4.67 & 0.58 & 4 & 5 \\
\hline
\end{tabular}

Change scores were calculated for each participant based on each individual's end of training and 4-5-month follow-up ratings. Change scores of 0 indicated no change in rating, while a positive change score indicated an increase in rating (i.e., their evaluation of the training after they were able to apply what they learned in a revenue-producing environment was better than their original assessment) and a negative change score indicated a decrease in rating (i.e., their opinion of the training method in those areas was worse than their original assessment). These scores are presented in Table 22. No distinct patterns can be determined based on the low sample size. However, the simulator training group had a greater number of participants, allowing for an examination of that group's trend in ratings over time. The simulator training group displayed very little change $(M=0.00, S D=0.38)$ in their rating of control skills training between 
measurement times. Likewise, very few differences in scores were observed between measurement times on training of safety skills $(M=0.13, S D=0.35)$.

Table 22. Change Scores for Rating of Training

\begin{tabular}{|l|l|r|c|c|r|r|}
\hline Training Group & Change Measure & \multicolumn{1}{c|}{$\boldsymbol{n}$} & Mean Change Score & SD & Minimum & Maximum \\
\hline Conventional & Control Skills & 6 & 1.17 & 0.41 & 1 & 2 \\
\hline Conventional & Safety Skills & 6 & 0.83 & 0.75 & 0 & 2 \\
\hline Simulator & Control Skills & 15 & 0.00 & 0.38 & -1 & 1 \\
\hline Simulator & Safety Skills & 15 & 0.13 & 0.35 & 0 & 1 \\
\hline Informal & Control Skills & 3 & 0.33 & 0.58 & 0 & 1 \\
\hline Informal & Safety Skills & 3 & 0.00 & 1.00 & -1 & 1 \\
\hline CDL-focused & Control Skills & 3 & 0.00 & 1.00 & -1 & 1 \\
\hline CDL-focused & Safety Skills & 3 & 0.33 & 0.58 & 0 & 1 \\
\hline
\end{tabular}

5.4.5 Research Question 3d: Would participants trained primarily on the simulator deem the simulator skill tests as a valid and fair assessment of their driving skills at the time they completed their training programs?

During the 4-5-month follow-up after being hired as a CDL driver, participants were asked if they felt that the Simulator and DTCC skills test (including both the road and range tests) were fair assessments of their driving skills at that time (appendix $G$ ). Participants provided ratings on a scale of 1-5, with 1 indicating that the tests were "extremely unfair" and 5 indicating that the tests were "extremely fair.” Group means are shown in Table 23.

Table 23. Group Means for Participant Rating of Tests as a Valid and Fair Assessment

\begin{tabular}{|l|l|r|r|r|r|r|}
\hline Training Group & \multicolumn{1}{|c|}{ Test } & \multicolumn{1}{c|}{$\boldsymbol{n}$} & \multicolumn{1}{c|}{ Mean } & \multicolumn{1}{c|}{ SD } & \multicolumn{1}{c|}{ Minimum } & Maximum \\
\hline Conventional & Simulated Skills Tests & 5 & 3.80 & 0.45 & 3 & 4 \\
\hline Conventional & BTW DTCC Skills Tests & 6 & 4.67 & 0.52 & 4 & 5 \\
\hline Simulator & Simulated Skills Tests & 15 & 4.40 & 0.74 & 3 & 5 \\
\hline Simulator & BTW DTCC Skills Tests & 15 & 4.47 & 0.83 & 2 & 5 \\
\hline Informal & Simulated Skills Tests & 3 & 4.33 & 0.58 & 4 & 5 \\
\hline Informal & BTW DTCC Skills Tests & 3 & 4.33 & 0.58 & 4 & 5 \\
\hline CDL-focused & Simulated Skills Tests & 3 & 3.00 & 1.00 & 2 & 4 \\
\hline CDL-focused & BTW DTCC Skills Tests & 3 & 4.00 & 1.00 & 3 & 5 \\
\hline
\end{tabular}

Note: One conventional training group participant was removed from the simulated skills tests analysis as he did not complete any simulator testing due to simulator sickness symptoms.

As shown, participants in the simulator training group had a mean rating of 4.40 on the simulated skills tests $(S D=0.74$; minimum $=3$, maximum $=5)$. This indicated that all participants in the simulator training group rated the simulated skills at a minimum of "fair" on the scale. Of these participants, one simulator training group participant (who assigned a rating of 2) was later terminated from his job due to a CMV rollover collision. 
Other possible explanatory factors for the ratings include participant test performance. Only one of the three informal training group participants passed the follow-up DTCC road testing and all three passed the DTCC range test. None of the informal training group participants passed the Simulator road test, while two passed the Simulator range test. None of the three CDL-focused training group participants passed either the DTCC or Simulator road tests. All three CDLfocused training group participants passed the DTCC range test, and one passed the Simulator range test. These participants' test performance may help better explain their ratings of the tests as a valid and fair assessment.

5.4.6 Research Question 3e: Would participants recommend their method of training to other prospective commercial driver trainees?

Participants were asked if they would recommend the method of training they experienced to other prospective entry-level drivers. Figure 15 shows the results from this question. Most participants from all groups would recommend their method of training to other prospective drivers.

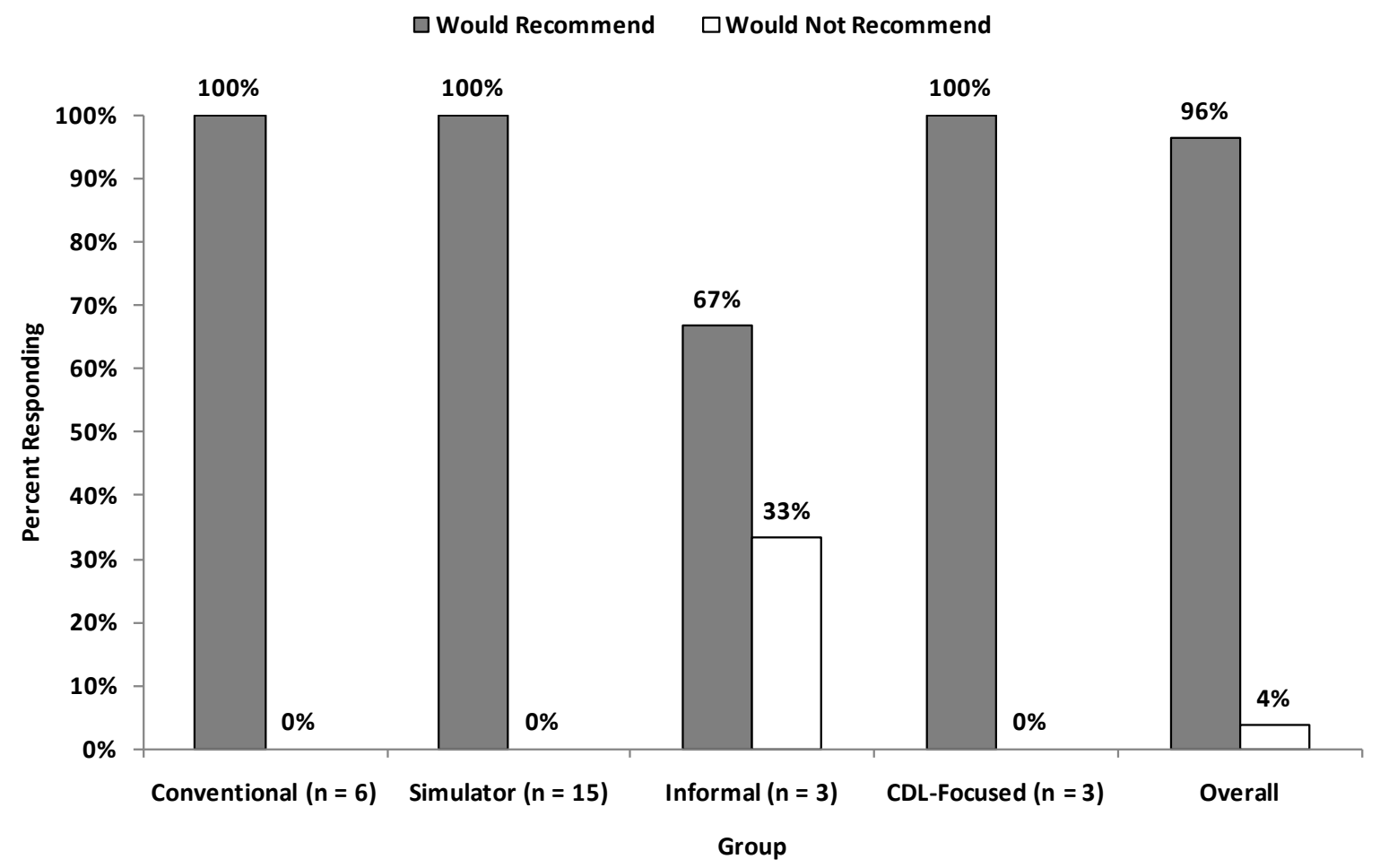

Figure 15. Chart. Participants' recommendations of training type.

As shown, all participants in the conventional, simulator, and CDL-focused training groups would recommend their training type to other potential drivers. One informal training group participant indicated that he would not recommend this training type to other potential drivers.

\subsubsection{Discussion}

Due to the economic downturn in the last few years, many participants were not able to find a job where they were required to use their Class A CDL. Therefore, the results presented herein were restricted due to a small sample. However, the trend presented in these findings seems to be 
consistent across all four training groups. All drivers thought they were better than average in terms of control and safety skills. Overall, drivers thought their training method was average or above average. Some drivers found potential deficiencies in their program after they were in a revenue-producing environment using their Class A CDL, but on average most ratings were no different from their original rating or better. However, every participant in the conventional group rated their training method equal or better than they originally rated it. When they were evaluated in a simulated environment, drivers thought that the simulator assessment was fair. Most of the drivers who participated in the study, and were currently employed as professional drivers, would recommend the training method they selected to obtain their entry-level training. Only one participant would not recommend the training method he selected (i.e., informal).

\subsection{TOPIC \#4: LONGITUDINAL EFFECTS OF TRAINING METHOD ON DRIVER PERFORMANCE}

As part of this section the following research question was answered:

5.5.1 Research Question 4a: Does training method affect performance on the simulated $\mathrm{road} / \mathrm{r}$ ange and DTCC road/range test during the retest 4-5 months after they start working as a commercial driver where a CDL is required? Furthermore, does the training method affect the magnitude of change in driving performance 4-5 months after receiving a CDL?

Methods, analysis, measures of interest, results, and discussion related to these questions are presented next.

\subsubsection{Method and Results}

Data from the testing 4-5-months after being hired were analyzed. Participants completed a replication of both DTCC and simulator road and range tests. A total of 22 participants completed the 4-5-month follow-up testing; however, one conventional training group participant experienced simulator sickness and did not complete any of the Simulator tests (thus was excluded from the present analysis). In a manner identical to data preparation for entry-level testing, a score of 50 percent was assigned to participants who failed a test (in either Simulator or DTCC road or range tests). This was done as participants were not allowed to complete testing once they scored below 75 percent; thus, it was unknown what their actual score would have been if they had been allowed to continue. The choice of 50 percent was made in an attempt to reduce the effect of extreme values (such as 0 percent) in the analysis while keeping a representative failing score. Due to the low number of participants that were employed utilizing a Class A CDL in their current employment, elaborated statistical analyses were not feasible. However, descriptive statistics are presented below for all the training groups.

The status of all entry-level participants is provided in Table 24. It should be noted that 15 participants (3 conventional group, 5 informal group, and 7 CDL-focused group) were unable to be contacted once reaching their 4-5-month date after employment. These participants did not respond to repeated attempts to contact by the onsite researcher. Additionally, four participants (two in conventional group and two in the simulator group) who completed the 4-5 month selfassessment did not show up for their scheduled meeting time to complete testing. These 
participants subsequently did not respond to repeated attempts to contact by the onsite researcher. One conventional group participant who was contacted declined further participation. Also, one informal group participant who was contacted and completed the self-assessment 4 months after being hired was unable to be scheduled for testing due to moving out of State and being employed as an over-the-road driver.

Table 24. Status of Participants for Follow-up Testing

\begin{tabular}{|l|c|c|c|c|}
\hline Training Group & $\begin{array}{c}\text { Participants } \\
\text { Completed Initial } \\
\text { Testing }\end{array}$ & $\begin{array}{c}\text { Participants } \\
\text { Eligible for } \\
\text { Follow-Up }\end{array}$ & $\begin{array}{c}\text { Participants } \\
\text { Screened for } \\
\text { Follow-Up }\end{array}$ & $\begin{array}{c}\text { Participants } \\
\text { Completed } \\
\text { 4-Month Testing }\end{array}$ \\
\hline Conventional & 33 & 10 & 7 & $4^{*}$ \\
\hline Simulator & 32 & 15 & 15 & 13 \\
\hline Informal & 9 & 8 & 3 & 2 \\
\hline CDL-focused & 33 & 10 & 3 & 3 \\
\hline
\end{tabular}

*One conventional group driver was driving a straight (Class-B) truck at the time of testing

As shown in Table 25, most groups displayed wide variations in performance on the follow-up tests. One participant in the conventional training group experienced simulator sickness and did not complete the Simulator road and range tests. Notably, none of the informal training group participants passed the simulator and DTCC road tests; however, both of these participants passed the DTCC and Simulator range exams.

Table 25. Descriptive Statistics for Follow-up Testing

\begin{tabular}{|l|l|r|r|r|r|r|}
\hline Training Group & \multicolumn{1}{c|}{ Test } & \multicolumn{1}{c|}{$\boldsymbol{n}$} & \multicolumn{1}{c|}{ Mean } & \multicolumn{1}{c|}{ SD } & \multicolumn{1}{c|}{ Minimum } & \multicolumn{1}{c|}{ Maximum } \\
\hline Conventional & DTCC Road & 4 & 76.75 & 18.79 & 50 & 94 \\
\hline Conventional & DTCC Range & 4 & 98.25 & 2.36 & 95 & 100 \\
\hline Conventional & Simulator Road & 3 & 59.00 & 15.59 & 50 & 77 \\
\hline Conventional & Simulator Range & 3 & 58.33 & 14.43 & 50 & 75 \\
\hline Simulator & DTCC Road & 13 & 79.46 & 14.25 & 50 & 95 \\
\hline Simulator & DTCC Range & 13 & 95.23 & 6.10 & 81 & 100 \\
\hline Simulator & Simulator Road & 13 & 77.85 & 14.00 & 50 & 92 \\
\hline Simulator & Simulator Range & 13 & 75.54 & 18.53 & 50 & 98 \\
\hline Informal & DTCC Road & 2 & 50.00 & 0.00 & 50 & 50 \\
\hline Informal & DTCC Range & 2 & 98.00 & 0.00 & 98 & 98 \\
\hline Informal & Simulator Road & 2 & 50.00 & 0.00 & 50 & 50 \\
\hline Informal & Simulator Range & 2 & 91.00 & 12.73 & 82 & 100 \\
\hline CDL-Focused & DTCC Road & 3 & 63.33 & 23.09 & 50 & 90 \\
\hline CDL-focused & DTCC Range & 3 & 96.67 & 4.93 & 91 & 100 \\
\hline CDL-focused & Simulator Road & 3 & 50.00 & 0.00 & 50 & 50 \\
\hline CDL-focused & Simulator Range & 3 & 63.67 & 23.67 & 50 & 91 \\
\hline
\end{tabular}

A pattern emerges when comparing participants' performance in testing at the conclusion of entry-level training. As shown in Figure 16, participants in the conventional group had lower 
scores at the 4-month follow-up as compared to tests at the conclusion of entry-level training. Participants in the simulator training groups had lower scores in both the Simulated road and DTCC road tests at the 4-month follow-up test compared to the same tests at the conclusion of entry-level training. The Informal and CDL-focused Training Groups did not show a similar pattern due to the number of participants who failed either test. These patterns should be viewed with the understanding of the small sample size of all groups.

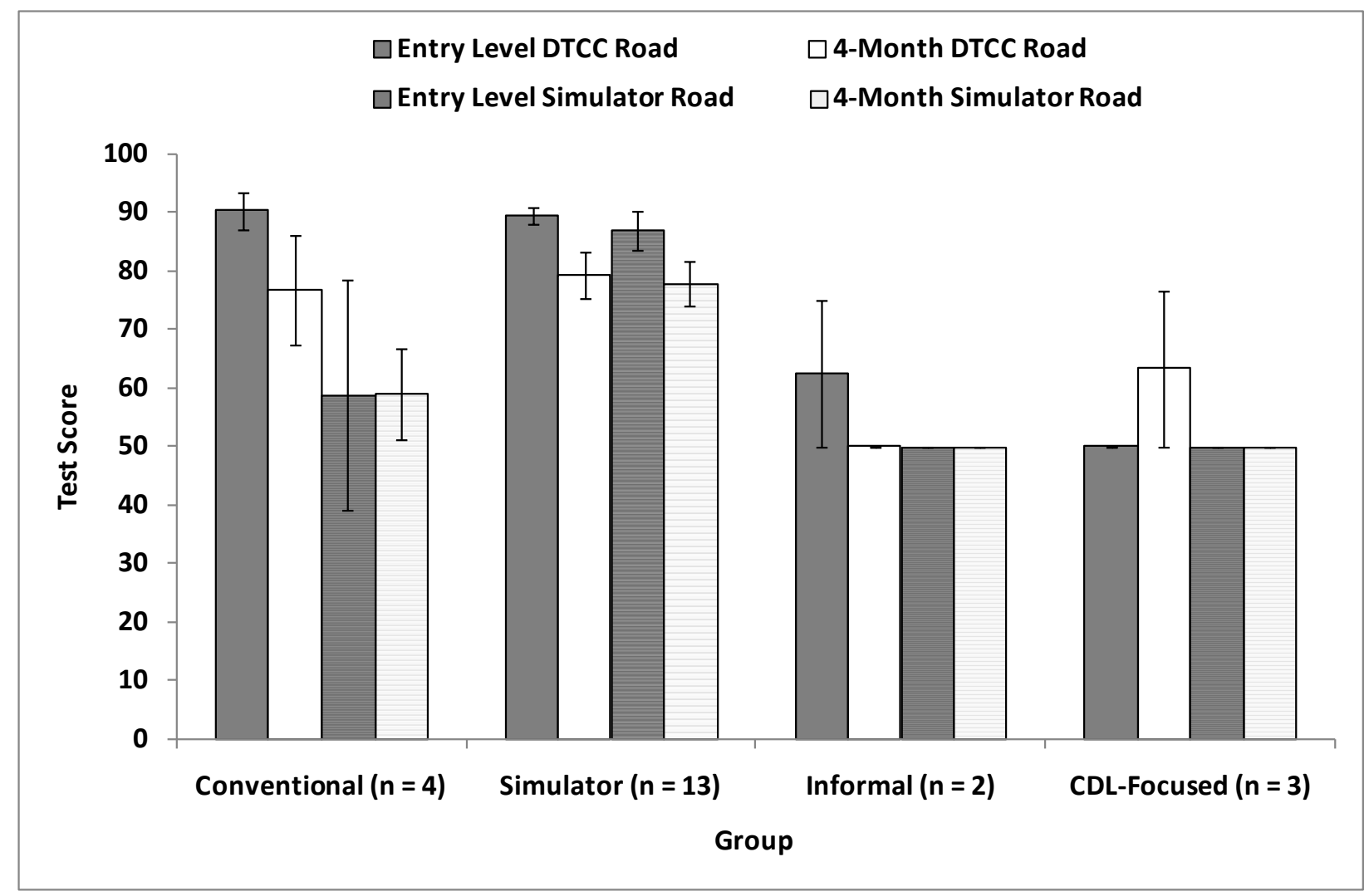

Figure 16. Chart. Road test: Entry level and follow-up scores by test mode and group.

Note: One conventional training group participant was not included in the Simulator test Error bars represent standard error.

A similar pattern was apparent in the examination of Simulator and DTCC range scores at the conclusion of entry-level training and the 4-month follow-up tests. As shown in Figure 17, the conventional, simulator, and CDL-focused training groups all displayed a drop in mean scores on the DTCC range test from the conclusion of entry level testing to follow-up testing. However, the informal training group's DTCC range test scores increased over this same period. The Conventional and simulator training groups both displayed a similar drop in Simulator range test scores. These results should be viewed with caution due to the small sample size of all groups. 


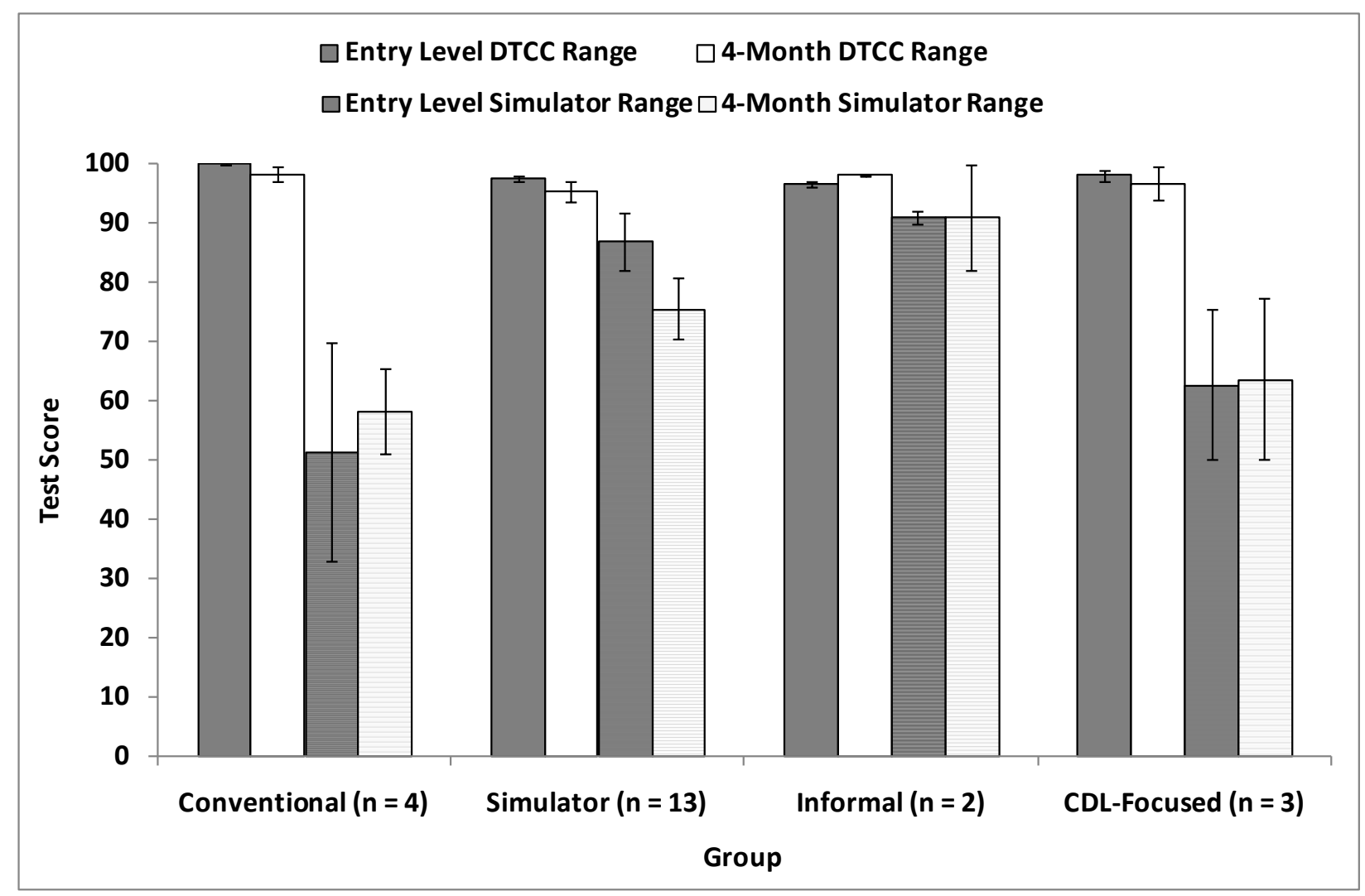

Figure 17. Chart. Range test: Entry level and follow-up scores by test mode and group. Note: One conventional training group participant was not included in the Simulator test Error bars represent standard error.

\subsubsection{Discussion}

The results obtained in the longitudinal follow-up were surprising. Most of the follow-up test scores were lower than the original scores during the entry-level tests. However, similar to the entry-level tests, the conventional and simulator groups performed better on the follow-up road tests than the Informal and CDL-focused groups. Possible explanatory factors for this are the transmission type of the participant's work vehicle, the type of backing maneuvers encountered on the job, and differences in driving exposure (i.e., local delivery versus over-the-road). A number of participants indicated that the trucks they were driving were equipped with an autoshift transmission or a synchronized transmission. Thus, these participants would not have continued practice and exposure to the task of shifting a double-clutching, non-synchronized, manual transmission and its associated sub-tasks (e.g., engine speed matching, gear selection, double clutching, use of range switches).

When compared to the shifting at the conclusion of entry-level training, shifting errors as an explanatory factor become more apparent. As shown in Table 26, participants in the conventional and simulator groups had an increase in the number of shifting errors at the 4month follow-up test as compared to at the conclusion of entry-level testing, while the CDLfocused group had a decrease in the number of shifting errors. 
Table 26. Entry-level and 4-month Follow-up Testing Shifting Errors (all 4-month participants)

\begin{tabular}{|l|l|r|r|r|r|r|r|}
\hline Training Group & \multicolumn{1}{|c|}{ Test } & $\boldsymbol{N}$ & $\begin{array}{c}\text { Total } \\
\text { Shifting } \\
\text { Errors }\end{array}$ & $\begin{array}{c}\text { Mean } \\
\text { Shifting } \\
\text { Errors }\end{array}$ & SD & Minimum & Maximum \\
\hline Conventional & Entry-Level DTCC Road & 4 & 19 & 4.75 & 3.77 & 0 & 9 \\
\hline Conventional & 4-Month DTCC Road & 4 & 41 & 10.25 & 7.27 & 3 & 20 \\
\hline Simulator & Entry-Level DTCC Road & 13 & 57 & 4.38 & 3.23 & 0 & 11 \\
\hline Simulator & 4-Month DTCC Road & 13 & 89 & 6.85 & 3.65 & 1 & 13 \\
\hline CDL-focused & Entry-Level DTCC Road & 3 & 55 & 18.3 & 7.64 & 10 & 25 \\
\hline CDL-focused & 4-Month DTCC Road & 3 & 33 & 11 & 11.79 & 1 & 24 \\
\hline
\end{tabular}

However, when examining only those participants from each group who drove an autoshift and synchronized transmissions (Table 27), it becomes apparent that participants who drive these partial or fully automated transmissions were responsible for the majority of shifting errors in follow-up testing. As shown in Table 27, the one conventional training group participant who drives an autoshift transmission accounted for 49 percent of the total shifting errors, while the five simulator training group participants accounted for 54 percent of the total shifting errors and the one CDL-focused training group participant was responsible for 73 percent of the total shifting errors. All informal training group participants who completed 4-month follow-up testing reported driving double-clutching, non-synchronized, manual transmissions at their place of employment. These results should be viewed with caution given the small sample size; however, the pattern seems to indicate deterioration in shifting proficiency on the part of those who only drive autoshift and synchronized transmissions.

Table 27. Entry-level and 4-month Follow-up Testing Shifting Errors

\begin{tabular}{|l|l|r|r|r|r|r|r|}
\hline \multicolumn{1}{|c|}{ Group } & \multicolumn{1}{c|}{ Test } & $\boldsymbol{n}$ & $\begin{array}{c}\text { Total } \\
\text { Shifting } \\
\text { Errors }\end{array}$ & $\begin{array}{c}\text { Mean } \\
\text { Shifting } \\
\text { Errors }\end{array}$ & SD & Minimum & Maximum \\
\hline Conventional & Entry-Level DTCC Road & 1 & 4 & 4 & - & - & - \\
\hline Conventional & 4-Month DTCC Road & 1 & 20 & 20 & - & - & - \\
\hline Simulator & Entry-Level DTCC Road & 5 & 23 & 4.6 & 4.16 & 1 & 11 \\
\hline Simulator & 4-Month DTCC Road & 5 & 48 & 9.6 & 2.61 & 7 & 13 \\
\hline CDL-focused & Entry-Level DTCC Road & 1 & 20 & 20 & - & - & - \\
\hline CDL-focused & 4-Month DTCC Road & 1 & 24 & 24 & - & - & - \\
\hline
\end{tabular}

Note: Includes only participants with autoshift or synchronized transmissions on their work vehicle.

Additionally, some participants may not encounter all of the CDL backing maneuvers (e.g., parallel parking) during their job duties. Thus, not having continued exposure to these skills would lead to deterioration in skill proficiency. Likewise, the lack of reinforcement for unsafe behaviors could have led to some participants' demerits during testing. For instance, some participants lost points for shifting during turns or removing a hand from the wheel during turns and three Simulator Group and three CDL-focused Group participants classified as local/delivery drivers received a total of five 5-point errors for speeding during the 4-month follow-up test. As participants no longer had an instructor in the passenger's seat of the truck to provide feedback, these unsafe behaviors could have become more frequent over the 4-month period. 


\subsection{TOPIC \#5: LONGITUDINAL EFFECTS OF TRAINING METHOD ON SAFETY PERFORMANCE BASED ON DRIVING RECORDS}

This research question sought to identify any relationships between training method and driver safety (as measured by the participants' driving records). The following research question was addressed:

5.6.1 Research Question 5a: Does training method affect safety performance on the job (e.g., incident rates, number of violations, number of crashes, supervisor ratings) after 4 and 12 months on the job?

The method, analysis, measures of interest, results, and discussion related to this question are presented next.

\subsubsection{Method}

Data were collected from each participant's driving record through the Delaware DMV and from the FMCSA CDLIS database. These participants' CDLIS records pertaining to the period of 1 year post-licensure were examined. Information on the number and type of violations was recorded, as well as whether the violation involved a CMV. Both citations (when the ticket was issued) and convictions (when the ticket was legally confirmed) were recorded. Note that if the field indicating the violation occurred while the participant was driving a CMV was left blank, the citation was recorded as a non-CMV citation by default. Thus, the true number of CMVinvolved citations may be greater than displayed below. All data entered into the CDLIS system as of February 11, 2010 were included in this analysis.

\subsubsection{Results}

CDLIS records were identified for all participants who were employed at the 4-month follow-up testing point. Note that this includes both the participants who were included in the follow-up performance testing (see Research Question 4) and participants who were employed as CMV drivers but were unable to participate in follow-up testing. The 44 drivers had nine violations; three of which involved a CMV. The distribution of drivers with violations was even among groups; however, due to the low sample size, only descriptive reports are provided (Table 28).

Table 28. Participant Violation Status by Group

\begin{tabular}{|l|c|c|}
\hline \multicolumn{1}{|c|}{ Training Group } & $\begin{array}{c}\text { Participants Employed at 4- } \\
\text { Month Follow-Up }\end{array}$ & $\begin{array}{c}\text { Participants from 4-Month Follow-Up } \\
\text { With Any Violation(s) }\end{array}$ \\
\hline Conventional & 10 & $2(20 \%)$ \\
\hline Simulator & 15 & $2(13 \%)$ \\
\hline Informal & 9 & $1(11 \%)$ \\
\hline CDL-focused & 10 & $2(20 \%)$ \\
\hline
\end{tabular}

Two participants in the conventional training group had recorded violations, neither was CMVrelated. In the simulator training group two participants had violations, both had CMV-related violations. One simulator training group participant had both CMV-related and general violations. One participant in the informal group had a CMV-related violation. The CDL-focused group had two participants with violations, neither of which were CMV-related violations. 
Details of each violation, along with the AAMVA Code Dictionary (ACD) code are provided in Table 29.

Table 29. Violations Recorded for All Follow-up Participants

\begin{tabular}{|l|c|c|c|c|l|}
\hline $\begin{array}{c}\text { Training } \\
\text { Group }\end{array}$ & $\begin{array}{c}\text { Number of } \\
\text { Parti- } \\
\text { cipants }\end{array}$ & $\begin{array}{c}\text { Days } \\
\text { between } \\
\text { Licensure } \\
\text { and Citation }\end{array}$ & $\begin{array}{c}\text { Violation } \\
\text { While } \\
\text { Driving CMV }\end{array}$ & $\begin{array}{c}\text { ACD } \\
\text { Code }\end{array}$ & \multicolumn{1}{|c|}{ ACD Code Description } \\
\hline Conventional & 4 & 107 & No & N04 & $\begin{array}{l}\text { Failure to yield to emergency } \\
\text { vehicle }\end{array}$ \\
\hline Conventional & 11 & 359 & No & S51 & Speeding: 01-10 > Speed limit \\
\hline Simulator & 53 & 149 & Yes & M17 & Failure to obey traffic sign \\
\hline Simulator & 70 & 52 & No & M19 & Failure to obey yield sign \\
\hline Simulator & 70 & 162 & Yes & S93 & Speeding \\
\hline Simulator & 70 & 184 & No & M17 & Failure to obey traffic sign \\
\hline Informal & 77 & 365 & Yes & S51 & Speeding: 01-10 > Speed limit \\
\hline CDL-focused & 109 & 42 & No & F04 & $\begin{array}{l}\text { Seat belt not used properly as } \\
\text { required }\end{array}$ \\
\hline CDL-focused & 118 & 108 & No & F04 & $\begin{array}{l}\text { Seat belt not used properly as } \\
\text { required }\end{array}$ \\
\hline
\end{tabular}

Additionally, four crashes were reported to the research team that did not appear in the CDLIS records. Four different participants accounted for these crashes. These crashes involved two conventional and two simulator training group participants. However, none of these crashes were police- or USDOT-reported and therefore no associated violation appeared in the CDLIS record. Two of these crashes (one each in the simulator and conventional groups) resulted in the involved driver being terminated from employment. The participant in the conventional training group was reassigned to the warehouse after his crash. Details of these crashes are summarized in Table 30. It should be noted that actual number of crashes could be greater as some crashes might not have been reported to the research team.

Table 30. Crashes Reported to Research Personnel

\begin{tabular}{|l|l|l|}
\hline \multicolumn{1}{|c|}{ Group } & \multicolumn{1}{c|}{ Crash Description } & \multicolumn{1}{c|}{ Crash Result } \\
\hline Conventional & Backed into a wall knocking off trailer door & Reassigned to warehouse \\
\hline Conventional & Backed into a store loading-dock wall & Terminated \\
\hline Simulator & Vehicle rollover & Terminated \\
\hline Simulator & Struck car with trailer while turning & Continued to drive \\
\hline
\end{tabular}

\subsubsection{Discussion}

The fact that the CDLIS records did not show the crashes is an important finding by itself. This type of database is used in many research efforts. However, as these finding reveal, some crashes are not reported or, under certain circumstances, not required to be reported; the fact that driving records may not show the true reason why a driver was terminated is important. There was limited data to determine differences in safety records between groups. However, based on the present results, all groups seem to have a similar number of violations per participant. 


\subsection{TOPIC \#6: LONGITUDINAL EFFECTS OF TRAINING METHOD ON SUPERVISOR EVALUATION OF EMPLOYEES}

As part of this section the following research question was addressed:

5.7.1 Research Question 6a: Does training method affect super visors' evaluation of their employees at 4-5 months after being hired and the number of DMV-reported and unreported incidents and violations?

The method, analysis, measures of interest, results, and discussion related to this question are presented next.

\subsubsection{Method and Results}

At the 4-5 month period after being hired, participants' supervisors and safety managers were contacted and asked to complete a brief questionnaire on each participant's on-the-job safety performance (appendix G). A total of 10 responses were obtained in three of the four training groups (conventional, simulator, and CDL-focused training groups). Summaries of responses received are provided below.

Prior to participation in the current study, all participants signed an authorization form allowing their future employer to release their records in the form of a supervisor evaluation. Once the participants reached their 4-month date of employment, their employers were contacted and a copy of the signed authorization was faxed, if requested. Despite multiple strategies were followed to have the participants' supervisor(s) assist in the evaluation process, supervisor onthe-job safety performance evaluations were not obtained for all participants. The main obstacle was multiple employers' refusal to complete any type of evaluation materials. Of the 43 eligible participants, only 10 employer evaluations were provided (2 conventional group, 6 simulator group, and 2 CDL-focused group). The number of eligible participants and the number of completed supervisor evaluations across all groups is shown in Table 31. As shown, employers refused to complete the evaluation for 24 participants. It should be noted that the 24 refusals do not represent 24 different employers. In addition, participants acting as owner-operators did not have a supervisor and therefore were not included. Three truckload carriers accounted for many of the refused evaluations. Each employer who refused to complete the evaluation noted liability concerns, even though the participant agreed to this release of data and despite being assured it was for research purposes only. Note that one participant in the conventional group declined further participation; therefore, his employer was not contacted. The employer for three participants in the CDL-focused group was not disclosed; thus, these three supervisors were unable to be contacted. 
Table 31. Supervisor Evaluations Availability, by Group

\begin{tabular}{|l|r|r|r|r|r|r|}
\hline $\begin{array}{c}\text { Training } \\
\text { Group }\end{array}$ & $\begin{array}{c}\text { Eligible } \\
\text { Participants }\end{array}$ & $\begin{array}{c}\text { Evaluations } \\
\text { Completed }\end{array}$ & $\begin{array}{c}\text { Participant } \\
\text { Dropped } \\
\text { Study or Did } \\
\text { Not Provide } \\
\text { Employer } \\
\text { Information }\end{array}$ & $\begin{array}{c}\text { Evaluations } \\
\text { Refused }\end{array}$ & $\begin{array}{c}\text { Owner } \\
\text { Operators }\end{array}$ & $\begin{array}{c}\text { Employer } \\
\text { Out of } \\
\text { Business }\end{array}$ \\
\hline Conventional & 10 & 2 & 1 & 5 & 2 & 0 \\
\hline Simulator & 15 & 6 & 0 & 8 & 1 & 0 \\
\hline Informal & 8 & 0 & 0 & 6 & 1 & 1 \\
\hline CDL-focused & 10 & 2 & 3 & 5 & 0 & 0 \\
\hline
\end{tabular}

No USDOT-reportable collisions were noted by supervisors. A total of three non-USDOTreportable collisions were reported, all in the simulator training group. Two of these nonUSDOT-reportable collisions were deemed preventable by the supervisors. Three moving violations were reported by the supervisors, one occurred in the simulator training group, while the other two occurred in the CDL-focused training group (Table 32).

Table 32. Frequency of Supervisor-reported Collisions and Violations, by Group

\begin{tabular}{|l|r|r|r|r|r|}
\hline Training Group & \multicolumn{1}{|c|}{$\begin{array}{c}\text { Evaluations } \\
\text { Completed }\end{array}$} & \multicolumn{1}{c|}{$\begin{array}{c}\text { DOT } \\
\text { Reportable } \\
\text { Collisions }\end{array}$} & $\begin{array}{c}\text { Non-DOT- } \\
\text { Reportable } \\
\text { Collisions } \\
\text { (Total) }\end{array}$ & $\begin{array}{c}\text { Non-DOT- } \\
\text { Reportable } \\
\text { Collisions } \\
\text { (Preventable) }\end{array}$ & $\begin{array}{c}\text { Number of } \\
\text { Moving } \\
\text { Violations }\end{array}$ \\
\hline Conventional & 2 & 0 & 0 & 0 & 0 \\
\hline Simulator & 6 & 0 & 3 & 2 & 1 \\
\hline Informal & 0 & N/A & 0 & 0 & N/A \\
\hline CDL-focused & 2 & 0 & 0 & 0 \\
\hline
\end{tabular}

Supervisor-reported miles driven by participants varied widely. The two participants in the CDLfocused training group had mileages of 8,696 and 120,000. However, one participant's supervisor provided mileage based on his original start date as a Class-B driver; thus, the 120,000 mileage figure includes the participant's previous 6 years as a local/short-haul Class-B straight-truck driver. The two conventional training group drivers had mileages of 37,232 and 52,000 , while the six simulator training group participants had an average number of 29,290 miles driven $(S D=9,772)$, with a range $1,200-65,842$ miles. This wide variation in miles driven can be partially attributed to the fact that some participants were working as local/short-haul drivers, while others were over-the-road/long-haul drivers. Note that two supervisors (one in the simulator training group, one in the CDL-focused training group) could not provide accurate miles-driven figures as their drivers were not required by USDOT regulations to keep a logbook. Based on supervisor-provided estimates of daily mileages (100 mi/day) and days in-service, estimates of 8,696 miles driven were used for these participants.

Supervisors were asked to compare the participant to other drivers of equal experience and rate the participant's basic control and maneuvering skills (control skills), and the participant's safety (e.g., defensive driving) skills. Ratings were provided on a scale of 1 ("One of the Worst") to 5 
(“One of the Best”). Overall, participants were rated between average and above average. The two conventional training group participants had control skill ratings of 3 and 4 , and safety skill ratings of 3 and 4, respectively. One simulator training group supervisor did not provide control or safety skill ratings; thus, the ratings in the simulator training group were based on five participants. The five simulator training group participants had a mean control skill rating of 3.6 $(S D=0.55$, range $=3-4)$ and a mean safety skill rating of $3.4(S D=0.55$, range $=3-4)$. The two CDL-focused training group participants had control skill ratings of 4 and 5 , and safety skill ratings of 2 and 4. The mean supervisor ratings for each of the groups are provided in Figure 18.

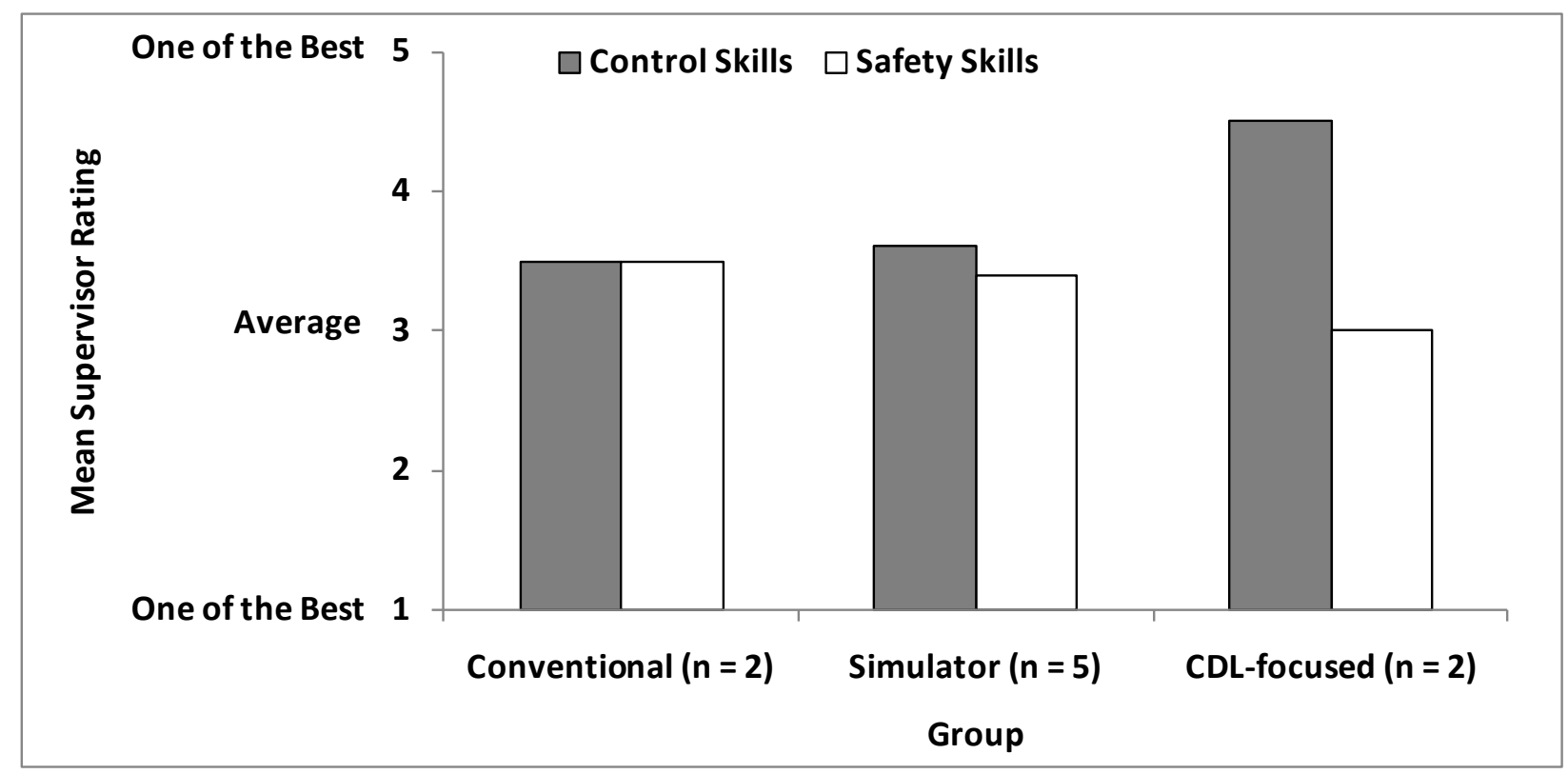

Figure 18. Chart. Supervisor ratings of participant control and safety skills.

Space was provided on the supervisor evaluation to comment on the driver. Only one supervisor reported on a participant. This was a supervisor of a CDL-focused training group participant, who noted, "He doesn't know how to switch out a trailer, which has created a problem. It is frustrating and surprising he graduated from school because of this very basic skill that he lacks.”

\subsubsection{Discussion}

No major differences existed in the ratings for the participants who were evaluated by their supervisors. All training groups received a control and safety skills rating above average with a tendency towards the side of best performance. Obtaining employee evaluations is becoming increasingly difficult due employers' concern over liability issues. As noted above, supervisors were reassured the evaluation was for research purposes and that their identity would be confidential; however, the response rate among supervisors completing the supervisor evaluation was only 29 percent. This lack of employer participation prevented further analysis. 


\subsection{TOPIC \#7: EXPLORATORY ANALYSIS OF “BIG FIVE” PERSONALITY CORRELATES}

This topic involved an exploratory analysis of the relationship between personality and aspects of training outcomes. As part of this section, the following research question was examined:

5.8.1 Research Question 7a: What is the relationship between the "Big Five” personality corr elates (i.e., openness, conscientiousness, extraversion, agr eeableness, and neur oticism) and forward transfer of training and longitudinal effects?

The method, analysis, measures of interest, results, and discussion related to this question are presented next.

\subsubsection{Method and Results}

The "Big Five" personality factor questionnaire (Goldberg, 1992) was administered to each participant. Note that, due to the small number of participants in the informal training group $(n=9)$, these were removed from this analysis. Following screening for data quality, internal consistency was assessed via Cronbach's alpha. Alpha, for each of the five factors, ranged between 0.82 and 0.85 . Factor correlations, with Cronbach's alpha reported on the diagonal are presented in Table 33.

Table 33. Correlations and Internal Consistency for Big Five factors

\begin{tabular}{|c|c|c|c|c|c|}
\hline Measure & Extraversion & Conscientiousness & Neuroticism & Openess & Agreeableness \\
\hline Extraversion & $\begin{array}{r}r=1 \\
\alpha=.85\end{array}$ & $\begin{array}{r}r=.49 \\
p<.0001\end{array}$ & $\begin{array}{r}r=.53 \\
p<.0001\end{array}$ & $\begin{array}{r}r=.51 \\
p<.0001\end{array}$ & $\begin{array}{r}r=.58 \\
p<.0001\end{array}$ \\
\hline Conscientiousness & - & $\begin{array}{r}r=1, \\
\alpha=.82\end{array}$ & $\begin{array}{r}r=.74 \\
p<.0001\end{array}$ & $\begin{array}{r}r=.52 \\
p<.0001\end{array}$ & $\begin{array}{r}r=.70 \\
p<.0001\end{array}$ \\
\hline Neuroticism & - & - & $\begin{array}{r}r=1, \\
\alpha=.83\end{array}$ & $\begin{array}{r}r=.50 \\
p<.0001\end{array}$ & $\begin{array}{r}r=.63 \\
p<.0001\end{array}$ \\
\hline Openness & - & - & - & $\begin{array}{r}r=1 \\
\alpha=.85 \\
\end{array}$ & $\begin{array}{r}r=.58 \\
p<.0001\end{array}$ \\
\hline Agreeableness & - & - & - & - & $\begin{array}{r}r=1, \\
\alpha=.83\end{array}$ \\
\hline
\end{tabular}

Means for each of the five factors were calculated and presented, by group, in Figure 19. 


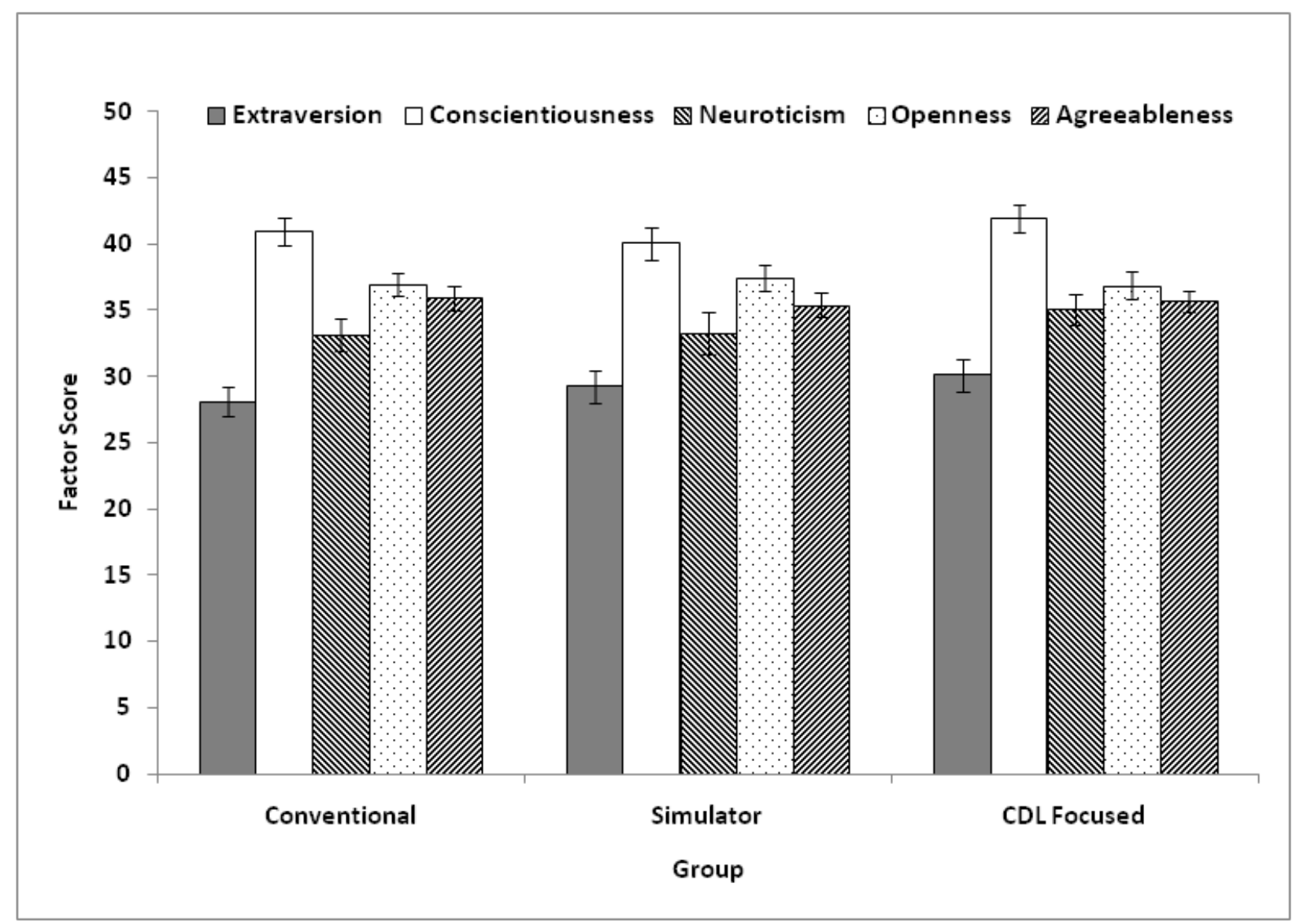

Figure 19. Chart. Personality factors, by groups.

Error bars represent standard error.

The personality factors were examined for group differences via one-way ANOVAs. No differences were found on personality factors based on group membership (Table 34); thus, the groups could be assumed to have a homogeneous make-up in terms of personality traits and the analysis proceeded using the combined results of the three groups.

Table 34. ANOVA Results for Personality Differences based on Group Membership

\begin{tabular}{|l|c|c|}
\hline \multicolumn{1}{|c|}{ Factor } & Significance Test & Result \\
\hline Extraversion & $F(2,95)=0.74, p=.48$ & Not significant \\
\hline Conscientiousness & $F(2,95)=0.71, p=.49$ & Not significant \\
\hline Neuroticism & $F(2,95)=0.62, p=.54$ & Not significant \\
\hline Openness & $F(2,95)=0.09, p=.91$ & Not significant \\
\hline Agreeableness & $F(2,95)=0.10, p=.90$ & Not significant \\
\hline
\end{tabular}

For each of the tests taken by participants (DTCC, Simulator, and DMV road and range tests), a standard multiple regression was performed between the outcome on these tests and the Big Five personality factors. In addition, group membership was included in the model (via two binary coded variables of conventional and CDL-focused training group membership, allowing for 
membership across the three training groups to be represented) due to the previously identified effect of group membership on outcomes of certain tests. In all cases except for the DMV road test, the models were significant (Table 35).

Table 35. Regression Model Results for Big Five Factors on Test Outcomes

\begin{tabular}{|l|c|c|c|}
\hline \multicolumn{1}{|c|}{ Test } & Model $\mathbf{R}^{2}$ & Significance Test & Result \\
\hline DTCC Road & $\mathrm{R}^{2}=.72$ & $F(7,90)=32.98, p<.0001$ & Significant differences \\
\hline DTCC Range & $\mathrm{R}^{2}=.40$ & $F(7,90)=8.74, p<.0001$ & Significant differences \\
\hline Simulator Road & $\mathrm{R}^{2}=.62$ & $F(7,82)=19.10, p<.0001$ & Significant differences \\
\hline Simulator Range & $\mathrm{R}^{2}=.40$ & $F(7,82)=7.93, p<.0001$ & Significant differences \\
\hline DMV Road & $\mathrm{R}^{2}=.07$ & $F(7,78)=0.78, p=.61$ & No significant differences \\
\hline DMV Range & $\mathrm{R}^{2}=.16$ & $F(7,78)=2.09, p=.05$ & Significant differences \\
\hline
\end{tabular}

Follow-up analysis revealed that, in all cases, group membership was the determining factor in the model reaching significance and none of the Big Five personality factors significantly explained any of the variability in test scores. In all cases, the parameter estimates for the Big Five personality factors failed to reach significance as a predictor of test performance ( $p \geq .07$ in all cases). Parameter estimates for group membership are provided in Table 36. As shown, membership in the CDL-focused training group was associated with a lower score on all range tests as well as on all road tests except for the DMV road test. Likewise, membership in the conventional training group was associated with lower performance on the simulator range test. 
Table 36. Training Group Membership Parameter Estimates for each Regression

\begin{tabular}{|l|c|c|c|c|c|}
\hline \multicolumn{1}{|c|}{ Parameter } & Test & Estimate & SE & $\boldsymbol{t}$ Value & $\boldsymbol{p}$ \\
\hline $\begin{array}{l}\text { 1. CDL-focused Training Group } \\
\text { Membership }\end{array}$ & DMV Range & -0.0357 & 0.0155 & -2.30 & 0.02 \\
\hline $\begin{array}{l}\text { 1. CDL-focused Training Group } \\
\text { Membership }\end{array}$ & DMV Road & -0.0109 & 0.0099 & -1.10 & 0.27 \\
\hline $\begin{array}{l}\text { 1. CDL-focused Training Group } \\
\text { Membership }\end{array}$ & DTCC Range & -0.2042 & 0.0322 & -6.35 & $<.0001$ \\
\hline $\begin{array}{l}\text { 1. CDL-focused Training Group } \\
\text { Membership }\end{array}$ & DTCC Road & -0.2974 & 0.0252 & -11.78 & $<.0001$ \\
\hline $\begin{array}{l}\text { 1. CDL-focused Training Group } \\
\text { Membership }\end{array}$ & Simulator Range & -0.2674 & 0.0403 & -6.63 & $<.0001$ \\
\hline $\begin{array}{l}\text { 1. CDL-focused Training Group } \\
\text { Membership }\end{array}$ & DMV Range & 0.0161 & 0.0141 & 1.14 & 0.26 \\
\hline $\begin{array}{l}\text { 2. Conventional Training Group } \\
\text { Membership }\end{array}$ & DMV Road & 0.0054 & 0.0090 & 0.60 & 0.55 \\
\hline $\begin{array}{l}\text { 2. Conventional Training Group } \\
\text { Membership }\end{array}$ & DTCC Range & 0.0091 & 0.0320 & 0.29 & 0.78 \\
\hline $\begin{array}{l}\text { 2. Conventional Training Group } \\
\text { Membership }\end{array}$ & DTCC Road & 0.0397 & 0.0251 & 1.58 & 0.12 \\
\hline $\begin{array}{l}\text { 2. Conventional Training Group } \\
\text { Membership }\end{array}$ & Simulator Road & -0.0509 & 0.0310 & -1.64 & 0.10 \\
\hline $\begin{array}{l}\text { 2. Conventional Training Group } \\
\text { Membership }\end{array}$ & $\begin{array}{l}\text { 2. Conventional Training Group } \\
\text { Membership }\end{array}$ & -0.1671 & 0.0405 & -4.13 & $<.0001$ \\
\hline
\end{tabular}

\subsubsection{Discussion}

The analyses performed on this research question were exploratory. No differences were found in any personality factor based on group membership, indicating the groups had very similar personality traits. As there were no group differences in any of the five factors, regression analyses were conducted to examine personality factor influences on test outcomes. No significant effects of personality were present in any of the tests. In all analyses, training group membership was the only significant factor in the model. This supports the findings in Topics 1 and 2 (the effects of training method on CDL and related test performance, and the validity of simulation training and test, respectively) above; therefore, skill test scores predicted training group membership and not personality factors.

\subsection{TOPIC \#8: ASSESSING ADVANCED CAPABILITIES OF THE SIMULATOR}

As part of this section the following research question was answered:

5.9.1 Research Question 8a: Are there differences in driver performance between million mile drivers and non-million mile drivers for the following types of advanced capability skills in a simulated environment:

Emergency maneuvers by million-mile and non-million-mile drivers corresponding to their experience level with different vehicle configurations (i.e., van, tankers, and doubles). 
Driving under hazardous or extreme road conditions by million-mile and non-million-mile drivers corresponding to their vehicle configuration experience.

The method, analysis, measures of interest, results, and discussion related to these two questions are presented next.

\subsubsection{Method}

A total of 48 Class A CDL drivers with differing levels of experience served as participants in this demonstration of the simulator's advanced capabilities. Participants recruited included "million milers" (drivers with at least 1 million miles of commercial driving logged without a safety incident) and non-million milers (those drivers with less than 1 million miles of commercial driving logged without a safety incident or drivers who have had an at-fault safety incident). Participants were recruited from companies located in Delaware and Eastern Shore of Maryland, through word-of-mouth recruiting, and advertisements placed in the local newspaper that is distributed one the Delmarva Peninsula. All participants had experience primarily in one of three trailer types: van, double, or tanker trailers. Participant demographics for the ACS participants are given in Table 37. 
Table 37. ACS Participant Demographics

\begin{tabular}{|l|c|c|c|c|c|c|}
\hline Driver Type & $\begin{array}{c}\text { Number of } \\
\text { Participants }\end{array}$ & $\begin{array}{c}\text { Million Miler } \\
\text { Status }\end{array}$ & $\begin{array}{c}\text { Mean } \\
\text { Age }\end{array}$ & Gender & $\begin{array}{c}\text { Mean Years } \\
\text { Experience }\end{array}$ & $\begin{array}{c}\text { Mean } \\
\text { Miles } \\
\text { Logged }\end{array}$ \\
\hline Van Trailer & 6 & Million Miler & 51 & $\begin{array}{c}6 \text { male, } \\
0 \text { female }\end{array}$ & 31.3 & $2,942,976$ \\
\hline Van Trailer & 10 & Non-Million Miler & 44 & $\begin{array}{c}9 \text { male, } \\
1 \text { female }\end{array}$ & 7.7 & 439,100 \\
\hline $\begin{array}{l}\text { Doubles } \\
\text { Trailer }\end{array}$ & 6 & Million Miler & 51 & $\begin{array}{c}6 \text { male, } \\
0 \text { female }\end{array}$ & 23.0 & $2,281,976$ \\
\hline $\begin{array}{l}\text { Doubles } \\
\text { Trailer }\end{array}$ & 10 & Non-Million Miler & 42 & $\begin{array}{c}10 \text { male, } \\
0 \text { female }\end{array}$ & 12.0 & 537,687 \\
\hline Tanker Trailer & 6 & Million Miler & 51 & $\begin{array}{c}6 \text { male, } \\
0 \text { female }\end{array}$ & 29.5 & $2,538,667$ \\
\hline Tanker Trailer & 10 & Non-Million Miler & 42 & $\begin{array}{l}10 \text { male, } \\
0 \text { female }\end{array}$ & 10.1 & 402,300 \\
\hline
\end{tabular}

Following the initial two 30-minute orientation drives in the simulator, participants then began driving the advanced capabilities showcase scenario. This scenario included routine driving on highway, rural, and city roads while also encountering the 12 emergency maneuvers and 10 extreme conditions. A description of each of the emergency maneuvers is provided in Table 38, and a description of each of the extreme conditions is provided in Table 39. The operational definitions for the scoring of all emergency maneuvers and extreme driving conditions can be found in appendix E. 
Table 38. Emergency Maneuvers

\begin{tabular}{|l|l|}
\hline \multicolumn{1}{|c|}{ Scenario Name } & \multicolumn{1}{c|}{ Scenario Description } \\
\hline Merge Squeeze & Vehicles merging from highway entrance ramp without yielding. \\
\hline Lane Cross & Oncoming vehicle crossing the center line. \\
\hline Tire Blowout & Driver will experience a steering axle tire blowout. \\
\hline Rollovers-Right & $\begin{array}{l}\text { Designated right hand curves that will cause a rollover event } \\
\text { unless speed is reduced below posted warning. }\end{array}$ \\
\hline Rollovers-Left & $\begin{array}{l}\text { Designated left hand curves that will cause a rollover event unless } \\
\text { speed is reduced below posted warning. }\end{array}$ \\
\hline Brake Failure & $\begin{array}{l}\text { Slow air pressure loss until brakes lock; warning light will activate } \\
\text { at } 60 \text { lbs of air pressure. }\end{array}$ \\
\hline Evasive Maneuver & $\begin{array}{l}\text { Vehicle abruptly stops in travel lane on highway forcing driver to } \\
\text { swerve into left lane or onto shoulder to avoid. }\end{array}$ \\
\hline Animal Crossing & Driver will encounter deer crossing while traveling on a rural road. \\
\hline Blind Entrance & Vehicle pulls out from a blind entrance. \\
\hline Pedestrian & Driver will encounter a child chasing a ball out into the road. \\
\hline Tight City Turns & $\begin{array}{l}\text { Turns causing the trailer to off-track into oncoming lane; traffic is } \\
\text { present. }\end{array}$ \\
\hline Roadway Obstruction & Driver will encounter a deer carcass in the travel lane. \\
\hline
\end{tabular}

Table 39. Extreme conditions

\begin{tabular}{|l|l|}
\hline \multicolumn{1}{|c|}{ Scenario Name } & \multicolumn{1}{c|}{ Scenario Description } \\
\hline Fog & Heavy fog while driving on the highway. \\
\hline Rain & Heavy rain with slick roads while driving on the highway. \\
\hline Snow & Snow-covered roads. \\
\hline Black Ice & Black ice encountered on highway and exit ramp. \\
\hline $8 \%$ Upgrade & Continuous 2-mile grade in snow. \\
\hline $8 \%$ Downgrade (Dry) & Continuous 2-mile grade in dry conditions. \\
\hline $\begin{array}{l}8 \% \text { Downgrade } \\
\text { (Snow) }\end{array}$ & Continuous 2-mile grade in snow. \\
\hline Dirt Road & Half mile in length with bumps. \\
\hline Construction Zone & $\begin{array}{l}\text { "Construction Ahead" signs followed by left lane closure on the highway and } \\
\text { reduced speed. }\end{array}$ \\
\hline Railroad Crossing & $\begin{array}{l}\text { Encountered when entering the town; signs and road marking, no crossing } \\
\text { gates. }\end{array}$ \\
\hline
\end{tabular}

Due to the complex nature of these simulated vehicles and environments, there were some occasions where the participant did not encounter the event (e.g., the driver's speed prevented simulated traffic from creating a merge squeeze) resulting in missing data. Participants were not asked to perform the maneuver(s) again in these situations. It should be noted that these situations occurred in less than 2 percent of the trials during the emergency maneuvers, and in less than 1 percent of the trials during extreme situations. 


\subsubsection{Results}

Participants received a categorical rating based on their driving response to each of the emergency maneuvers and extreme conditions. The ratings for all 48 drivers were assigned by the same experimenter and included "responded appropriately," "responded inappropriately," and "did not respond/collision." Overall, the majority of participants from all truck types and experience levels responded appropriately to the emergency maneuvers (Figure 20). The lowest number of appropriate responses was seen in the tanker trailer, non-million-mile participants, who responded appropriately to 57.3 percent of the events. The highest level of appropriate responses was seen in van trailer, million-mile participants, who responded appropriately in 80 percent of the events.

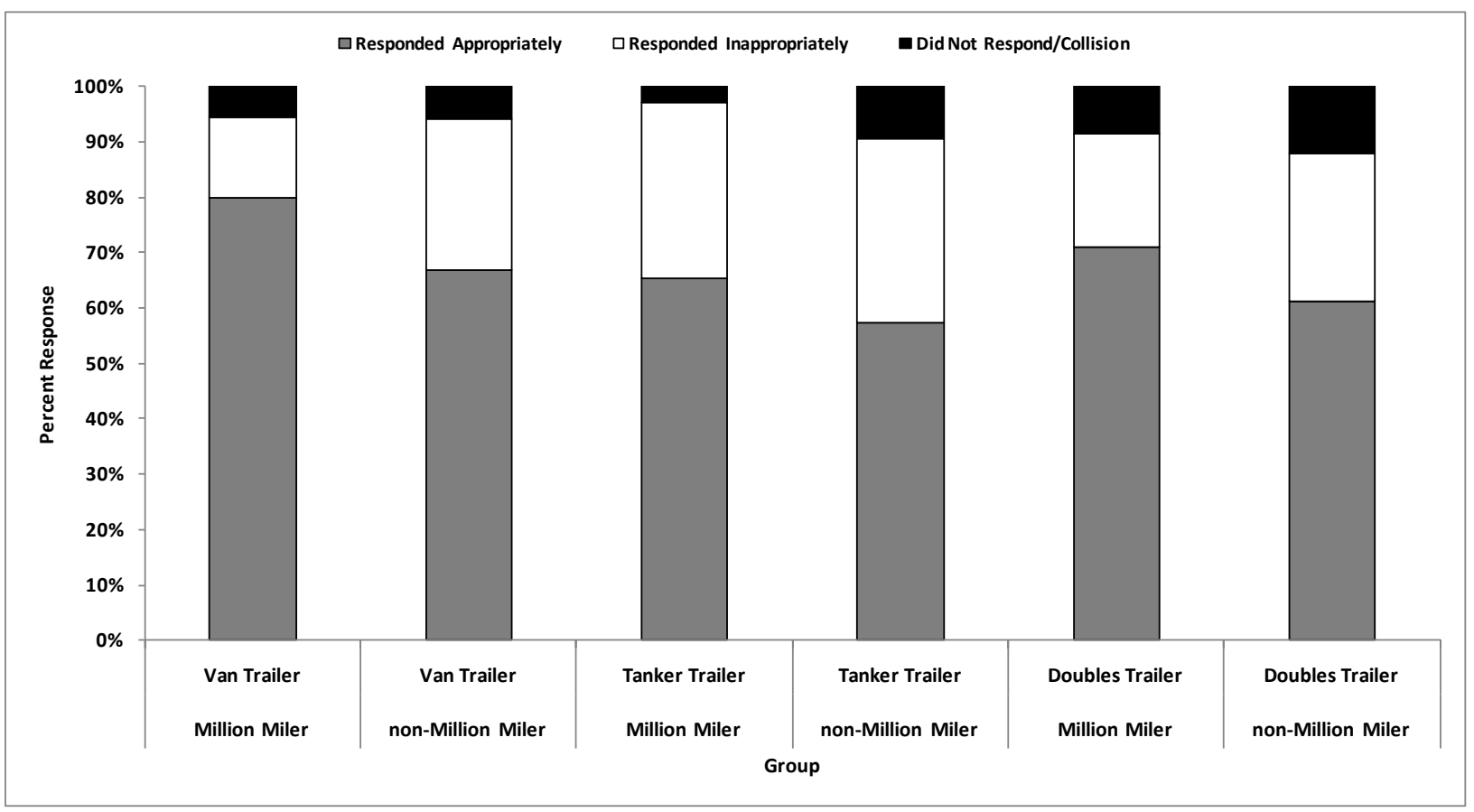

Figure 20. Chart. Overall participant responses to emergency maneuvers.

Similar to the participant responses to the emergency maneuvers, the majority of all participant groups responded appropriately to the 10 extreme conditions (Figure 21). The lowest percentage of appropriate responses was seen in van trailer, non-million-mile participants (54.6 percent), while the highest percentage of appropriate responses was seen in tanker trailer, million-mile participants (73.3 percent). 


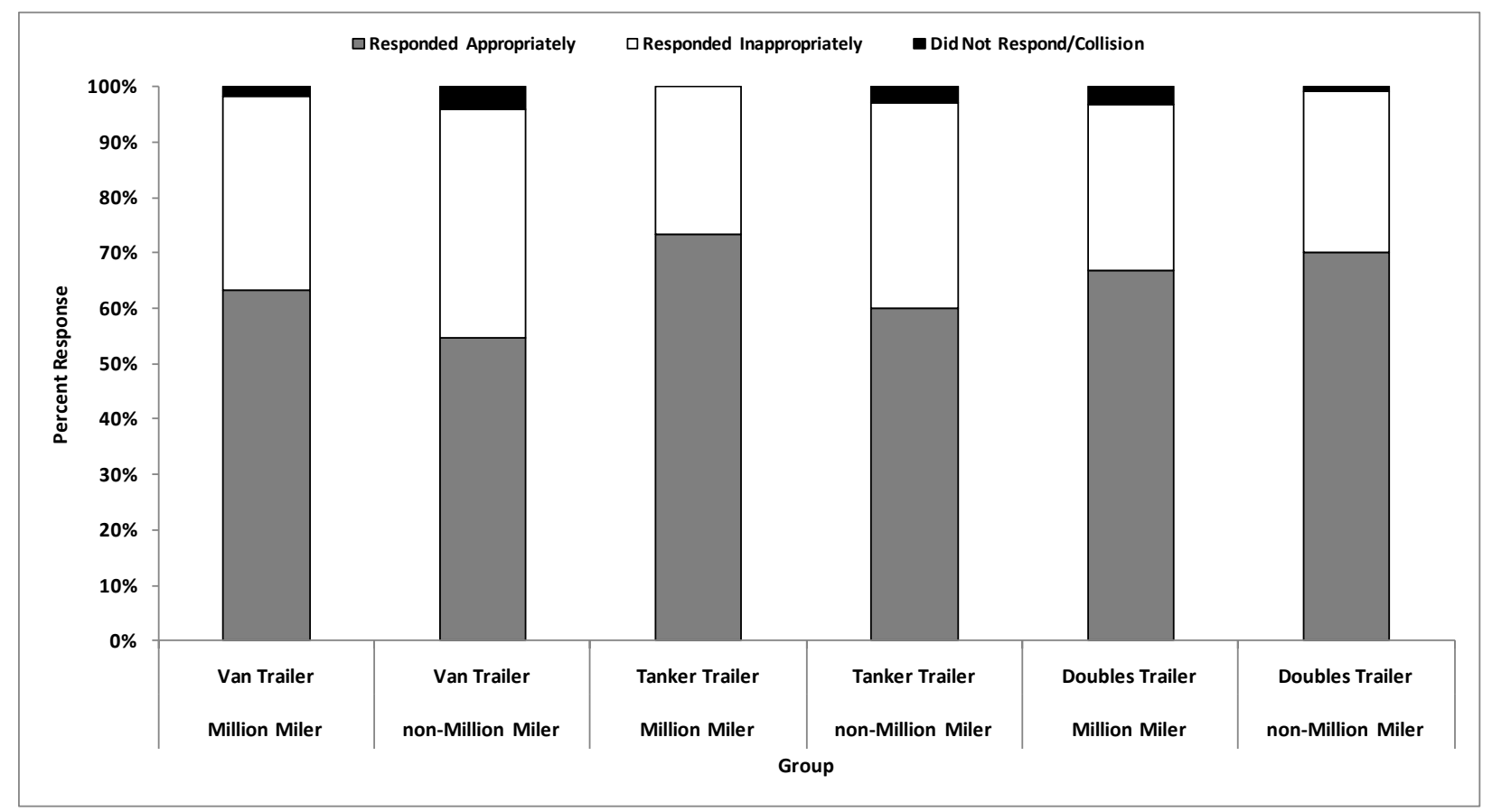

Figure 21. Chart. Overall participant responses to extreme conditions.

As indicated above, each participant experienced 12 emergency maneuvers and 10 extreme conditions. Comparisons between the responses, based on ratings assigned by the experimenter, of million-mile and non-million-mile participants were evaluated. Fisher's exact statistical significance test was used to evaluate the 12 emergency maneuvers. Results indicated a significant difference between tanker trailer million milers and non-million milers during the offroad recovery scenario ( $p=0.035$ ), as well as between van trailer million milers and non-million milers during the tire blowout event $(p=0.035)$. Tanker trailer million milers were more likely to respond appropriately than non-million milers during the off-road recovery scenario. Van trailer million milers were more likely to respond appropriately than van trailer non-million milers during tire blow out scenarios. No other comparison reached statistical significance

The 10 extreme conditions were similarly examined using Fisher's exact test. Results indicated a significant difference between doubles trailer million milers and non-million milers during the black ice extreme condition $(p=0.036)$. In this black ice scenario, doubles trailer non-million milers were more likely than doubles trailer million miler doubles participants to respond appropriately. No other comparison reached statistical significance.

\subsubsection{Discussion}

No major statistical differences were found in the expected driving performance, as rated by the experimenter, of million-mile participants and non-million milers during the showcase scenarios. The overall performance during the 12 emergency maneuvers and 10 extreme conditions demonstrates that a majority of the participants responded to the scenario appropriately. Overall, million milers responded appropriately to the emergency maneuvers and extreme conditions more often than non-million milers. However, there some interesting results are present when each set of conditions were evaluated in detail. 
Extreme Conditions: While driving the simulator, participants encountered 10 different extreme conditions. The extreme driving conditions were designed to replicate many of the conditions CMV drivers may encounter on a daily basis while driving a tractor trailer in the real world. While both million milers and non-million milers tended to respond appropriately in these scenarios, the million milers tended to respond appropriately more often than the non-million milers. Million milers, across all trailer configurations, responded appropriately approximately 68 percent of the time, while the non-million milers responded appropriately approximately 62 percent of the time. However, when observing the extreme conditions on an individual basis, a rather limited number of these conditions produced the majority of the inappropriate responses for both million milers and non-million milers. These specific extreme conditions of interest were the railroad crossing, black ice, 8 percent upgrades, and 8 percent downgrades.

The railroad crossing was presented as the participants were entering a town in the simulated environment. The non-million-mile doubles participants were the only group to respond appropriately more than 70 percent of the time. An examination of the responses in this scenario found that an improper behavior was used for all of the scenarios where the participant received a rating of either inappropriate or no response/collision. One of these inappropriate behaviors was shifting while crossing the railroad tracks, which led one million-mile doubles participant to miss a gear and come to a stop with the trailers still on top of the railroad tracks. Additionally, three non-million-mile tanker participants did not perform a visual search or come to a stop before crossing the tracks. These findings are surprising, as it was expected that the million milers would have more appropriate responses to this scenario than non-million milers.

The black ice scenario was presented as participants were on a highway exit ramp. This extreme condition proved difficult to represent accurately in the simulated environment. Visual cues typically used by participants were unable to be replicated on the simulator. Participants provided some comments regarding these missing cues, noting that they typically see ice accumulate on their antennas and mirrors, and road spray gradually disappears on a wet road surface in conditions where black ice is likely. Thus, these participants were unable to determine the road conditions and adjust their driving accordingly.

The 8 percent downgrade scenario was presented on both a snow-covered roadway and a dry roadway. In both of these situations, participants performed inappropriate behaviors. Both million-mile and non-million-mile participants for all trailer configurations were up-shifting through gears while on the downgrade. This behavior can lead to speeding and a loss of control of the vehicle and result in the occurrence of a collision. One non-million-mile doubles participant and one non-million-mile van trailer participant experienced a crash. It was also observed that a number of participants over-revved the engine during the downgrades. This can be attributed to the sensory cues the simulator did not provide. Participants noted they typically use the sound of the engine and the "road feel" transmitted through the seat as a cue rather than strictly watching the tachometer.

The majority of inappropriate responses observed in the 8 percent upgrade condition were due to participants not using the four-way flashers while traveling less than $45 \mathrm{mi} / \mathrm{h}$. One possible explanation for this may be attributed to the physical location where simulated testing occurred. There are no upgrades or downgrades of any significance in this area (eastern Delaware and Maryland) and the participants may not routinely drive significantly under the posted speed limit. 
Of the million-mile participants, the tanker participants had the highest rate of appropriate responses (73.3 percent), while million-mile van trailer participants had the lowest rate of appropriate responses (63.3 percent). This trend is of interest as the dynamics of each trailer type is substantially different. Tanker trailer dynamics typically present the most challenging conditions for a participant, followed by the doubles trailers and van trailer. It would be expected that million-mile tanker participants should have a higher degree of vehicle awareness than those participants who have only pulled van trailers.

Emergency Maneuvers: While driving the simulator, participants encountered 12 different situations requiring emergency maneuvers. These situations were designed to assess the participant's ability to perform emergency maneuvers while driving. While appropriate responses were observed across both million milers and non-million milers in the majority of trials, million milers tended to respond appropriately more often than the non-million milers. Across all trailer configurations, million milers responded appropriately approximately 70 percent of the time, while the non-million milers responded appropriately approximately 62 percent of the time. When examining these emergency maneuvers individually, five maneuvers stand out, including brake failure (i.e., air pressure loss), front tire blowout, pedestrian encounter, merge squeeze (i.e., traffic merging onto highway), and the off-road recovery (i.e., evasive action).

Participants encountered a simulated brake failure event while traveling on the highway. Only one participant across all groups and trailer types responded appropriately. Participants were observed to fail to visually scan their gauges routinely and did not realize they were losing air pressure until the warning alarm was activated. Several participants noted they rarely use any of the gauges because they drive by the sound and "road feel" of the truck. However, one participant commented that this was valuable refresher training, as it forced him to use his gauges.

The majority of million-miles and non-million-miles participants responded inappropriately to the front tire blowout. These participants were observed to apply hard brake pressure immediately when the front tire blowout was encountered. While this may be the participants' first reaction, it is the incorrect action to take. The proper response for a front tire blowout is to use a smooth and steady application to the accelerator to maintain control of the vehicle. Once it is safe to do so, the driver is supposed to slowly bring the vehicle to a stop. Immediately applying hard brake pressure may cause sudden loss of control resulting in lane departure and/or a crash.

The pedestrian encounter was a scenario involving a person chasing a ball into the street in a simulated town/local road with a posted speed limit of $25 \mathrm{mi} / \mathrm{h}$. Eight participants (one nonmillion-mile van trailer, four non-million-mile doubles, two million-mile doubles, and one million-mile tanker) failed to stop in time and hit the pedestrian. In each instance, the participant was driving too fast for conditions. This inappropriate behavior may be explained by a lack of visual search and safe operations to assess the potential road hazards. Visual cues were provided for this scenario which prompted the majority of the participants to reduce speed below the posted speed limit. While these eight participants only account for 16.7 percent of the inappropriate responses in this scenario, these safety-critical events resulted in the simulated fatality of the pedestrian. 
Only non-million milers responded incorrectly to the merge squeeze situation. In this situation, traffic was merging onto the highway and failing to yield the right-of-way to the participant. Five participants' responses resulted in a crash. These participants did not perform an adequate visual search to assess the traffic hazards. One explanation for this lack of visual searching prior to the merge was the lack of defensive driving techniques refresher training. Only one of these participants (non-million-mile doubles participant) reported having an annual safety class and refresher training at his/her place of employment.

Participants also encountered a situation that required them to make an evasive maneuver to avoid a collision with another vehicle. Participants could use three actions to avoid the collision: perform a hard braking maneuver to safely bring the vehicle to a complete stop in the current travel lane, move onto the right shoulder, or move into the left lane. Both million-mile and nonmillion-mile participants for all trailer configurations experienced crashes. The nature of this type of emergency event would not be considered an at-fault crash; however, most employers would classify this as preventable. During these scenarios, participants did not perform regular traffic checks or visual searches. Seven non-million-mile tanker participants experienced a crash during this emergency situation; however, it should be noted that several of these participants commented that the safety manager at their place of employment instructed them never to swerve in this type of situation due to the increased risk of a rollover with tanker trailers.

Of the million-mile participants, those who pulled a van trailer had the highest rate of appropriate responses (80 percent), while million-mile tanker trailer participants had the lowest rate of appropriate responses (approximately 65 percent). This follows an expected trend as tanker trailer dynamics typically present the most challenging conditions for a driver, followed by the doubles trailers and the van trailer, respectively. It would be expected that million-mile participants who pull a van trailer should have a higher degree of vehicle handling and control skills than those participants who pull tanker trailers, as van trailers are the most stable of the three trailers used for this showcase.

The results of this showcase demonstrated that million-mile participants responded appropriately more often than non-million-mile participants during the emergency maneuvers and extreme conditions; however, the million-mile participants still responded inappropriately or not at all in approximately 30 percent of the emergency events and 32 percent of the extreme conditions encountered. These results indicate that all participants, including million milers, could potentially benefit from refresher and defensive-type training. The simulator provides one such mechanism for providing this type of training. As noted in the Case Study of Existing Driver Training Programs (section 7), some larger carriers are beginning to use driving simulators for this purpose. Thus, the simulated scenarios described here could be used as part of a training program for such refresher and defensive driving training.

\subsection{TOPIC \#9: PARTICIPANTS' RATINGS OF THE VARIOUS ASPECTS OF THE ADVANCED CAPABILITIES OF THE SIMULATOR.}

As part of this topic, two research questions were answered: 
5.10.1 Research Question 9a: How do both million-mile and non-million-mile drivers rate the usefulness and realism of the simulator for the presentation of the advanced capabilities?

5.10.2 Research Question 9b: Which emergency maneuvers, driving conditions, or other events provided by the simulator are most important and valuable to drivers, and which are rated as the most realistic by the drivers?

The method, analysis, measures of interest, results, and discussion related to these two questions are presented next.

\subsubsection{Method}

Following completion of the 12 emergency maneuvers and 10 extreme conditions (detailed in the preceding research question, within Topic 8), participants were asked to provide ratings of the simulator's re-creation of the situation as compared to that situations as experienced in a real truck (appendix J). Participant provided ratings on a Likert-type scale that ranged from 1 ("Extremely Different") to 5 (“Extremely Similar"). In addition, participants provided rankings of the top three most effective and top three least effective aspects of the simulator.

\subsubsection{Research Question 9a: How do both million-mile and non-million-mile drivers rate the usefulness and realism of the simulator for the presentation of the advanced capabilities?}

In two separate questions, participants were asked to rank the top three most and least effective aspects of the simulator. The choices given to participants included safety, repeatability, standardization, realism, enrichment, basic control, vehicle handling, and safe operations. These were the same aspects entry-level participants used to rate the simulator (see appendix E for operational definitions). Participants were not required to provide absolute ranks and could select up to three "top choice." Additionally, the selection of a simulator aspect as most effective did not prevent the same participant from selecting the same aspect as least effective. Thus, to analyze the participant rankings, a total weighted score was calculated for each aspect. This weighted score was based on the number of times an aspect was rated as \#1 (10 point weighting), \#2 (9 point weighting) or \#3 (8 point weighting), allowing for a single most-effective and single least-effective ranking score.

Million-mile doubles participants ranked safety as the most effective aspect of the simulator, followed by safe operations and enrichment. Million-mile doubles participants ranked only two aspects as least effective (standardization and basic control were tied for least effective). Similar results were found in non-million-mile doubles participants, who ranked realism as the most effective aspect, followed by safety and safe operations, respectively. Non-million-mile doubles participants ranked vehicle handling as the least effective aspect, followed by realism and enrichment. Doubles participants rankings of most and least effective aspects of the simulator are provided in Figure 22 and Figure 23, respectively. 


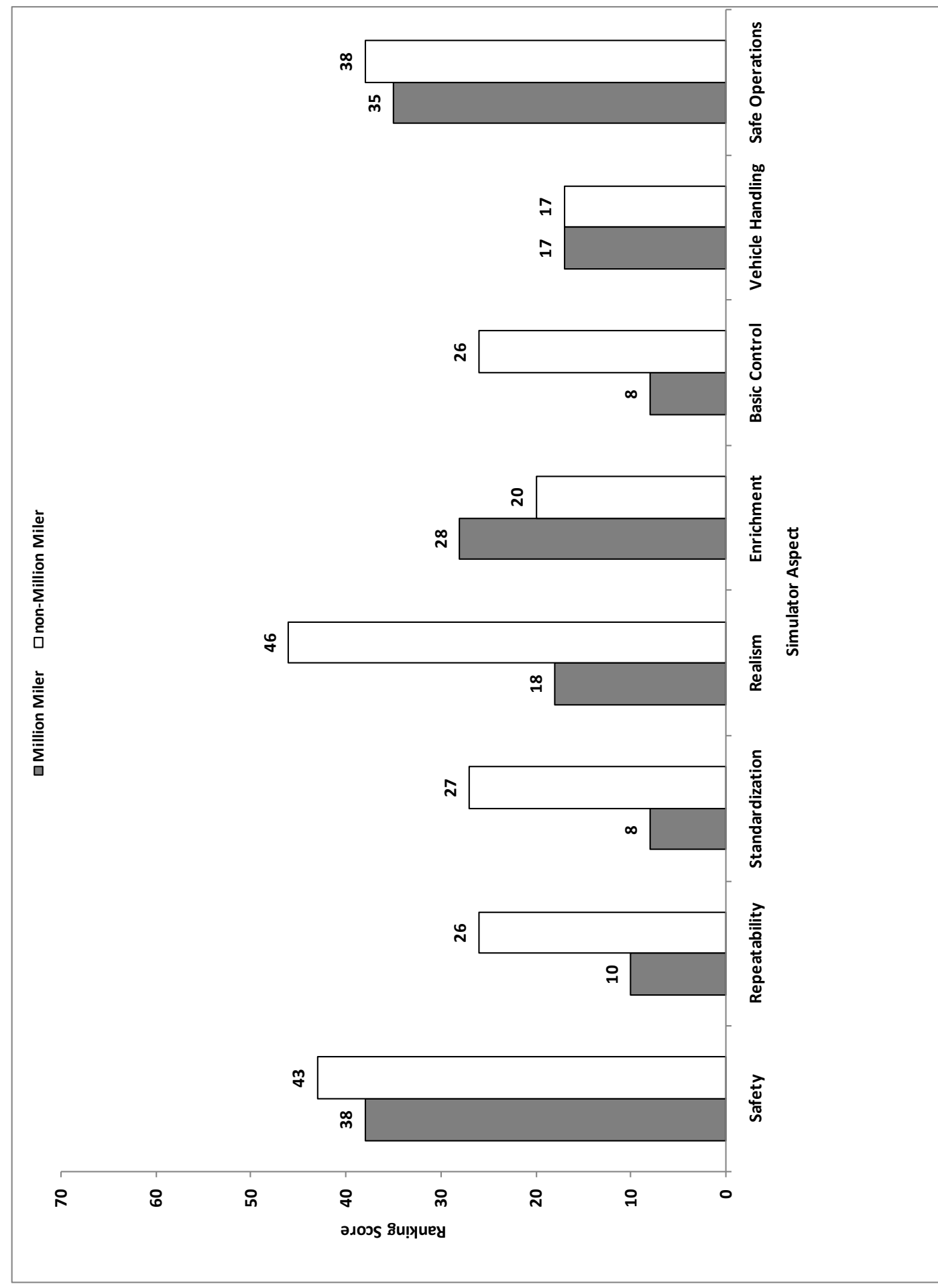

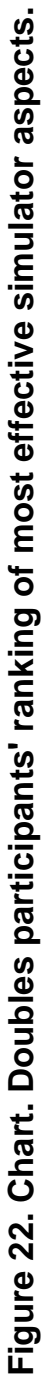




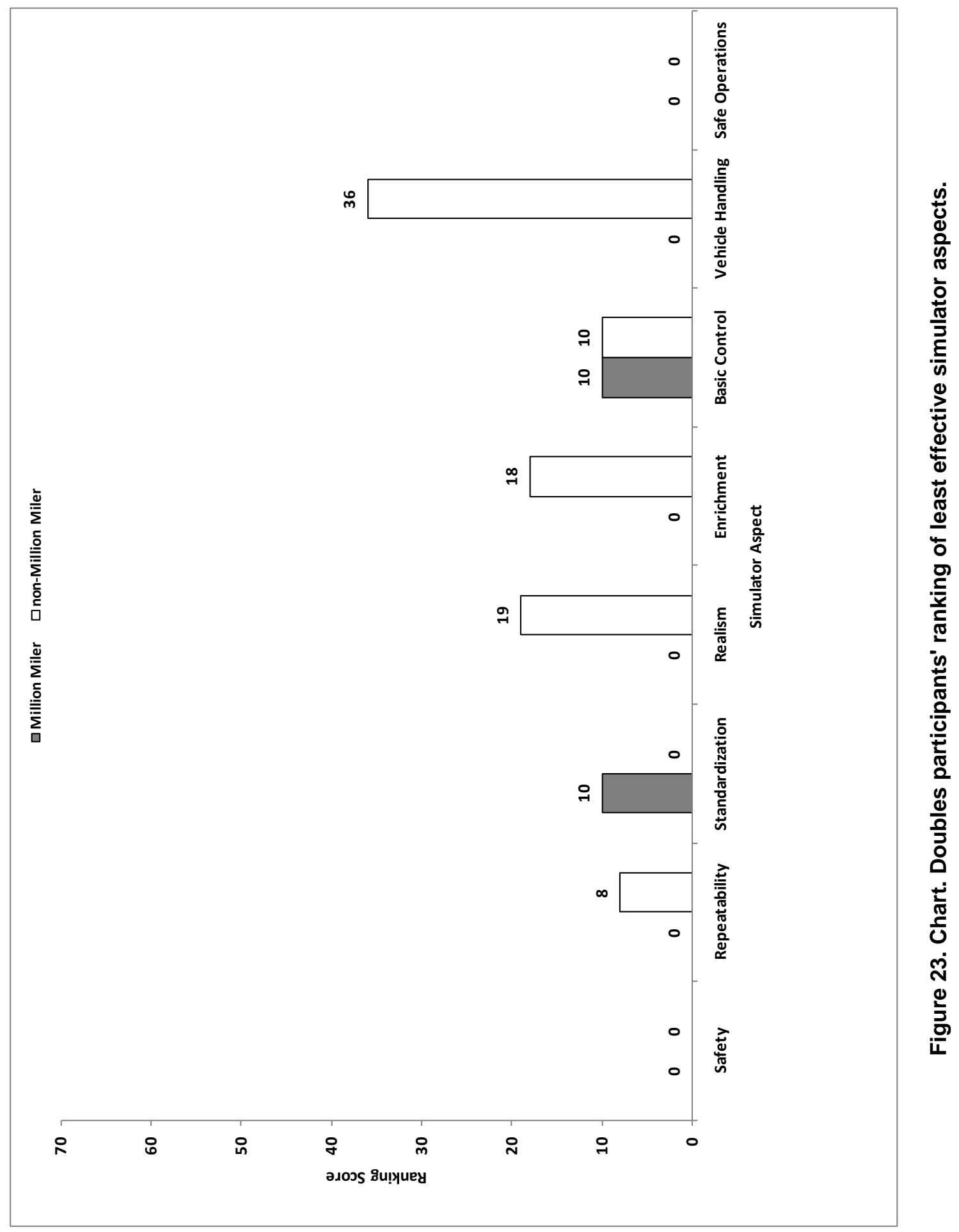


Million-mile tanker participants found realism as the most effective aspect of the simulator, followed by safety and safe operations, respectively. Million-mile tanker participants ranked enrichment as least effective, followed by realism and repeatability. Non-million-mile tanker participants ranked safe operations as the most effective aspect of the simulator, followed by safety and vehicle handling, respectively. Non-million-mile tanker participants ranked standardization as least effective, followed by repeatability and basic control and vehicle handling (tied for third least effective). Tanker trailer participants' rankings of most and least effective aspects of the simulator are provided in Figure 24 and Figure 25, respectively.

Million-mile van trailer participants ranked safety as the most effective aspect of the simulator, followed by realism and basic control (tied for second most effective) and repeatability. Millionmile van trailer participants rated basic control as the least effective aspect of the simulator, followed by vehicle handling and realism, respectively. Non-million-mile van trailer participants ranked enrichment as the most effective aspect of the simulator, followed by realism and safe operations, respectively. Non-million-mile van trailer participants ranked vehicle handling as the least effective aspect, followed by repeatability, and realism and safe operations (tied for the third least effective). Van trailer participants' rankings of most and least effective aspects of the simulator are provided in Figure 26 and Figure 27, respectively. 


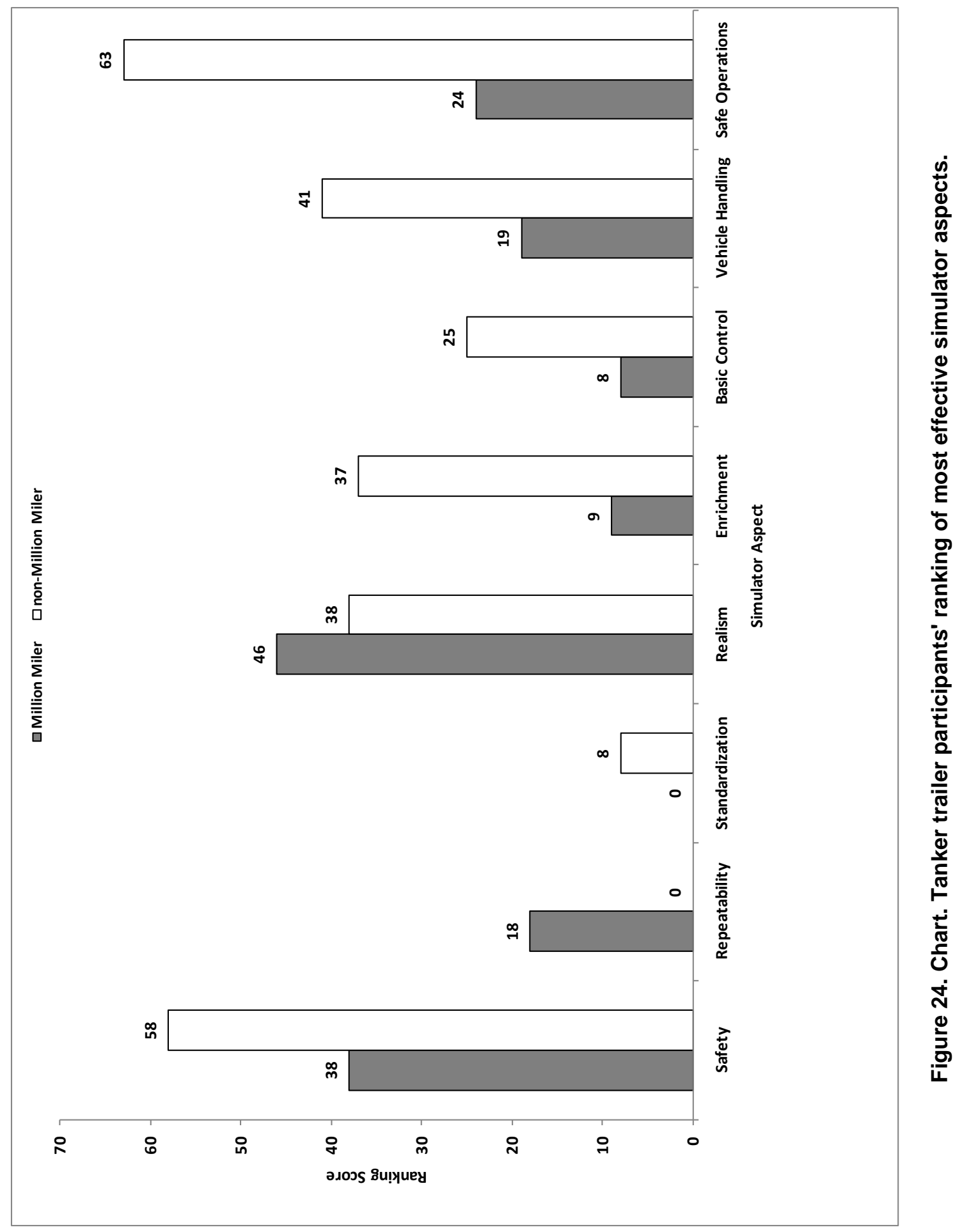




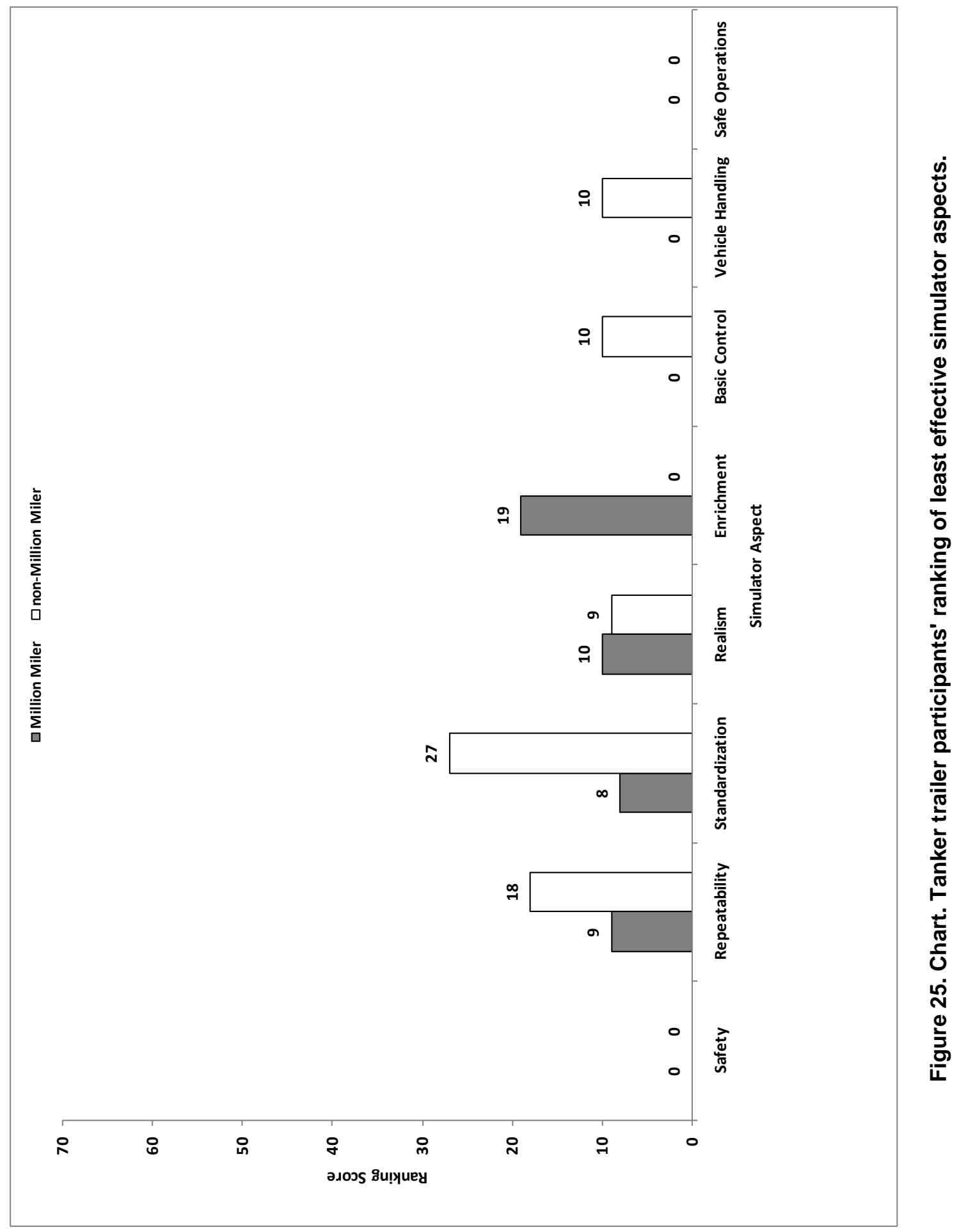




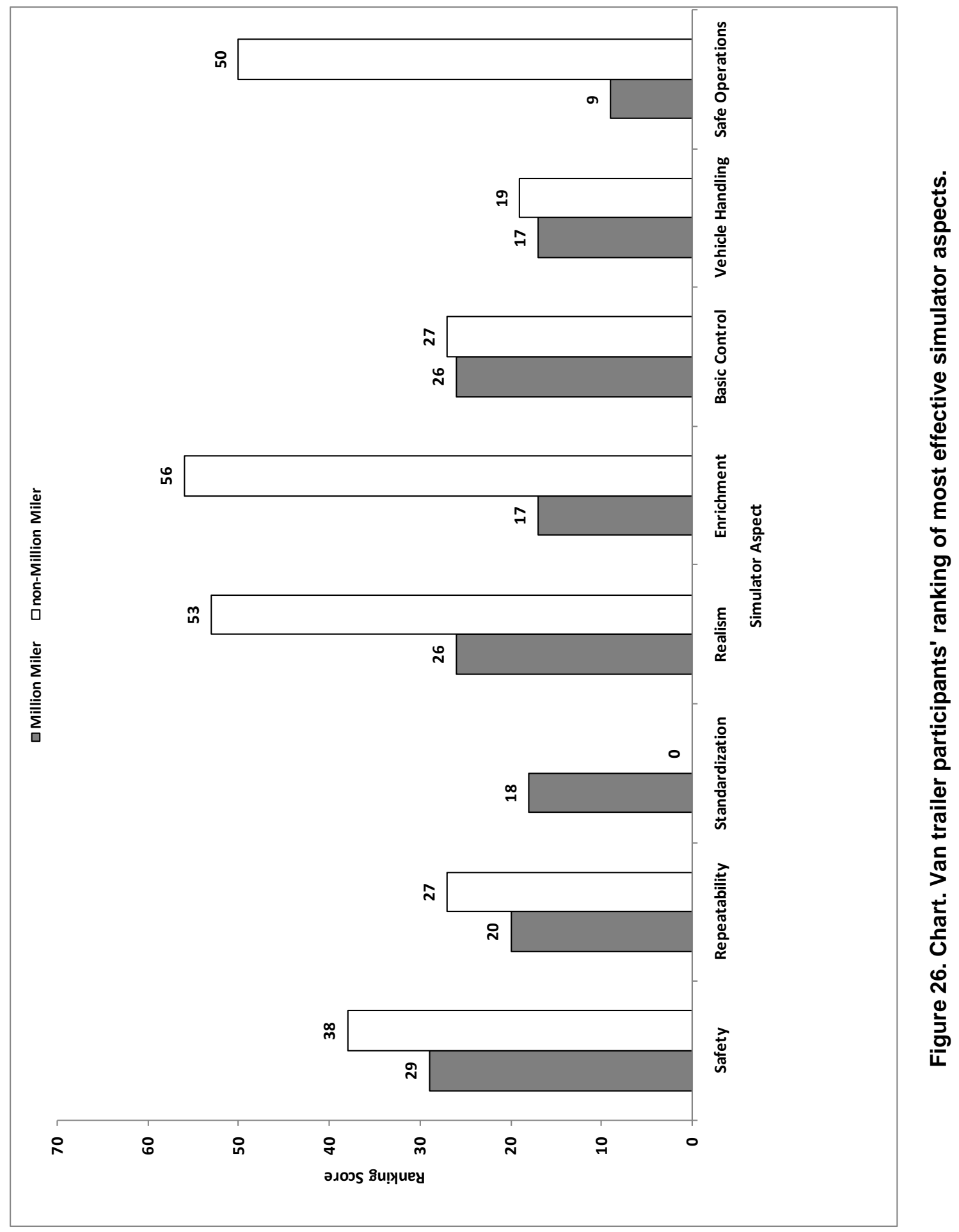




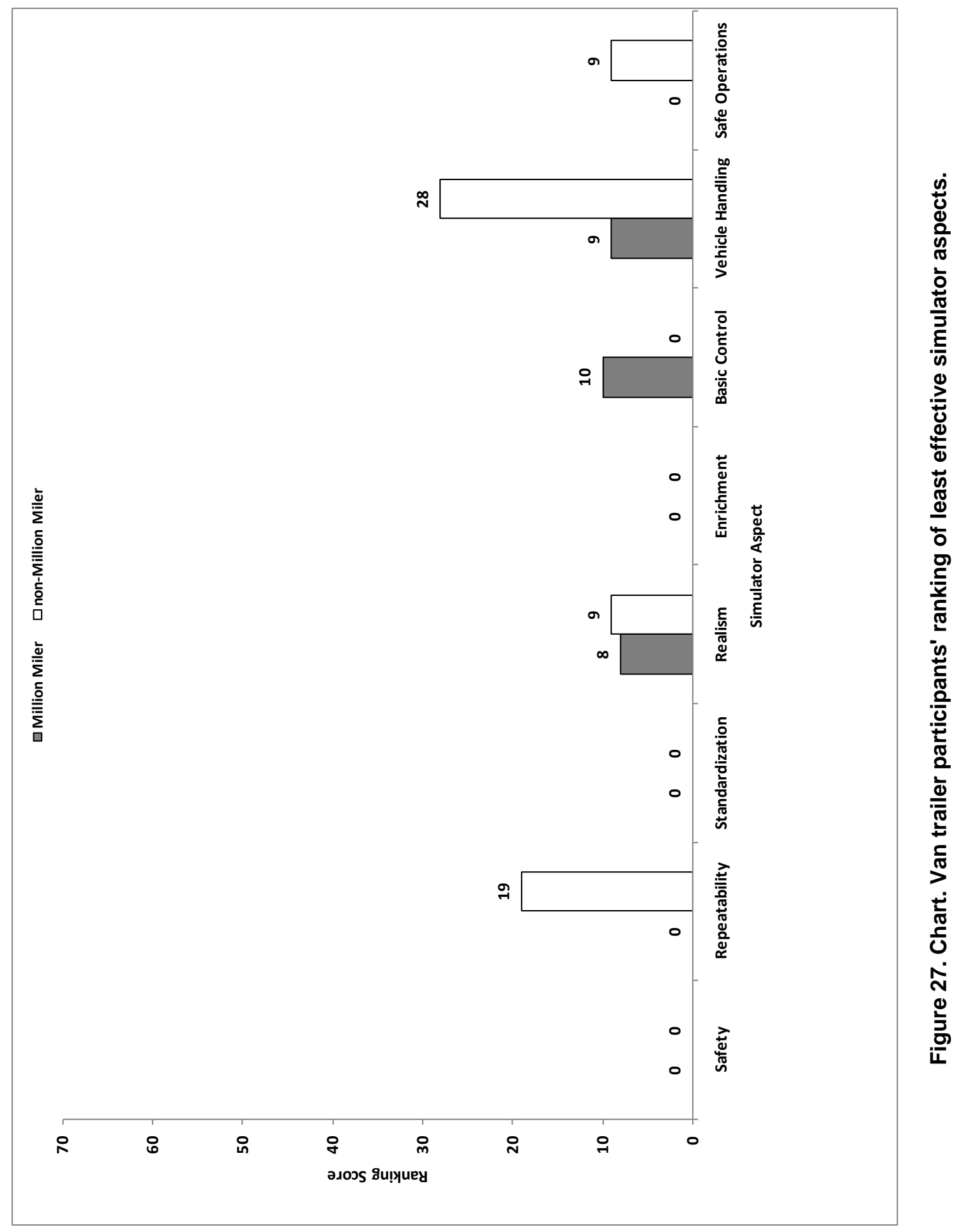




\subsubsection{Research Question 9b: Which emergency maneuvers, driving conditions, or other events provided by the simulator are most important and valuable to drivers, and which are rated as the most realistic by the drivers?}

Average ratings were calculated for each of the 12 emergency maneuvers and 10 extreme conditions based on driver experience level. Million miler ratings were compared to non-million miler ratings using Kruskal-Wallis chi-square tests. Results indicated that million milers and non-million milers typically provided similar ratings to each emergency maneuver and extreme condition, regardless of whether the participant was a van, doubles, or tanker trailer driver. The only conditions in which participants demonstrated a statistically significant difference in ratings were two emergency conditions: right rollovers for doubles participants $\left(\chi^{2}(1)=4.02, p=0.045\right.$ ), where million milers rated the scenario higher than non-million milers, and evasive maneuvers for tanker participants $\left(\chi^{2}(1)=4.03, p=0.045\right)$, where non-million milers rated the scenario as higher than million milers. Emergency maneuver ratings for doubles, tankers, and van trailer participants are shown in Figure 28, Figure 29, and Figure 30, respectively. Extreme condition ratings for doubles, tankers, and van trailer participants are shown in Figure 31, Figure 32, and Figure 33, respectively. 


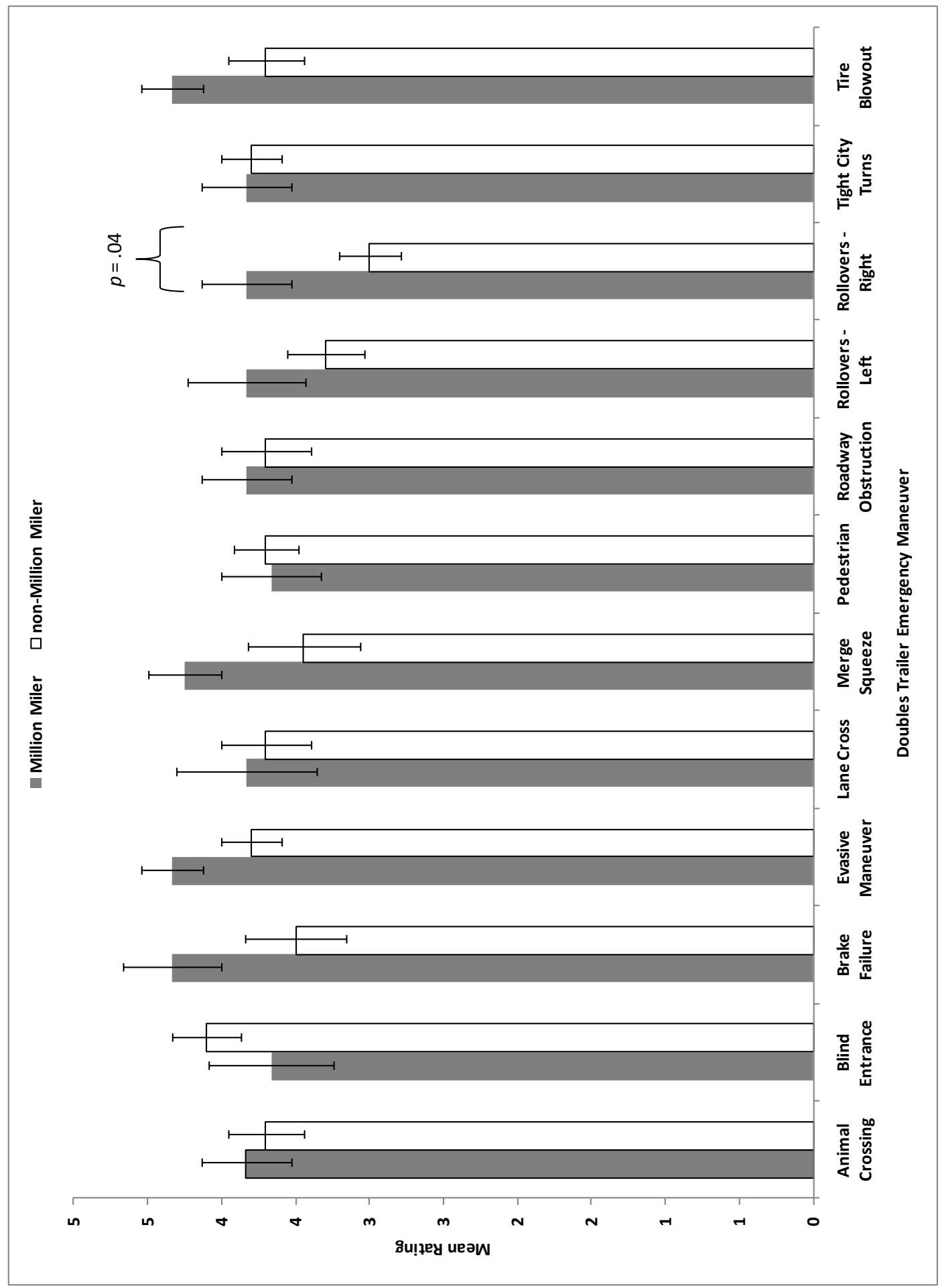

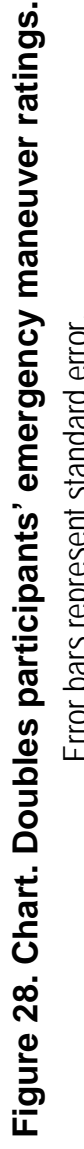




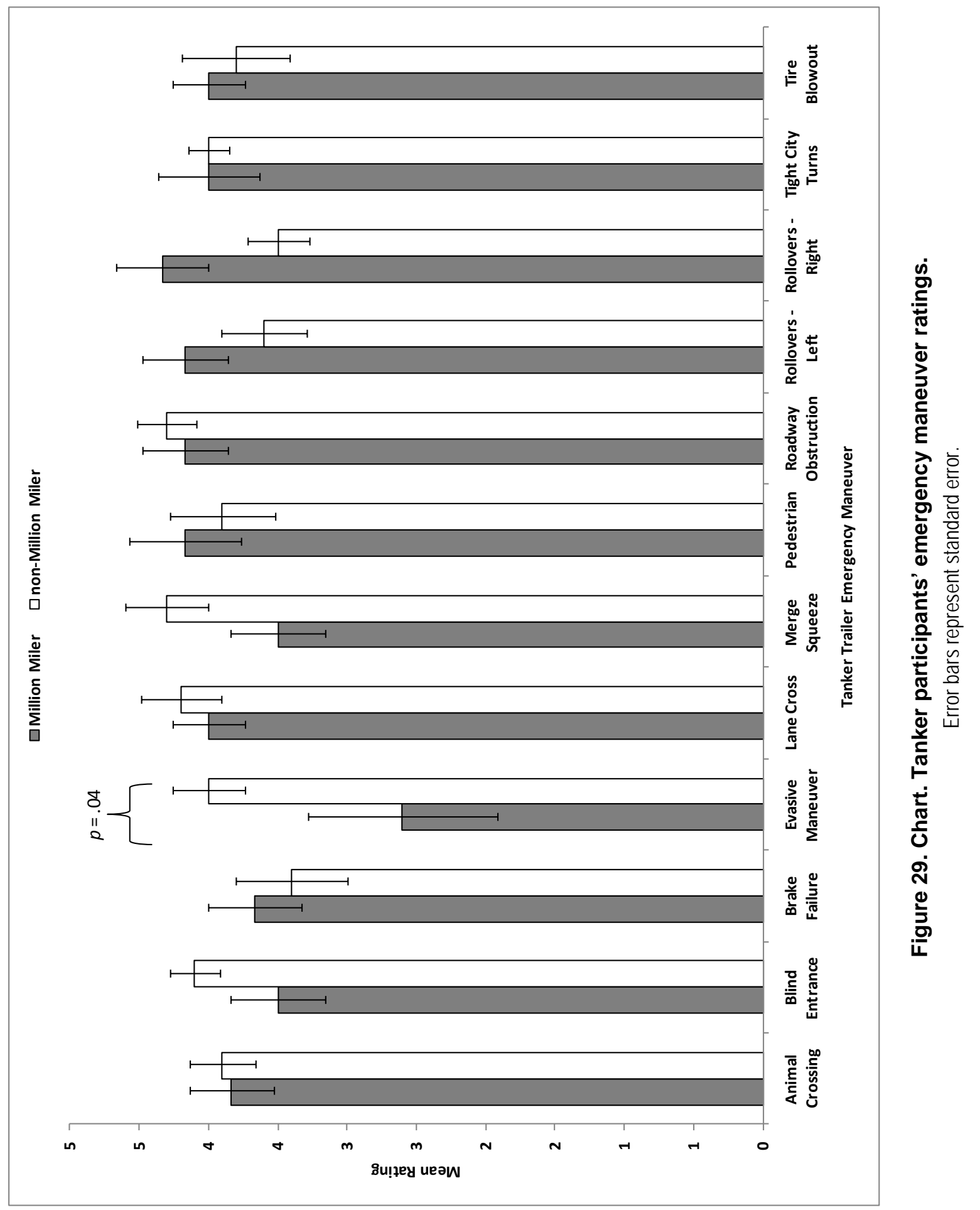




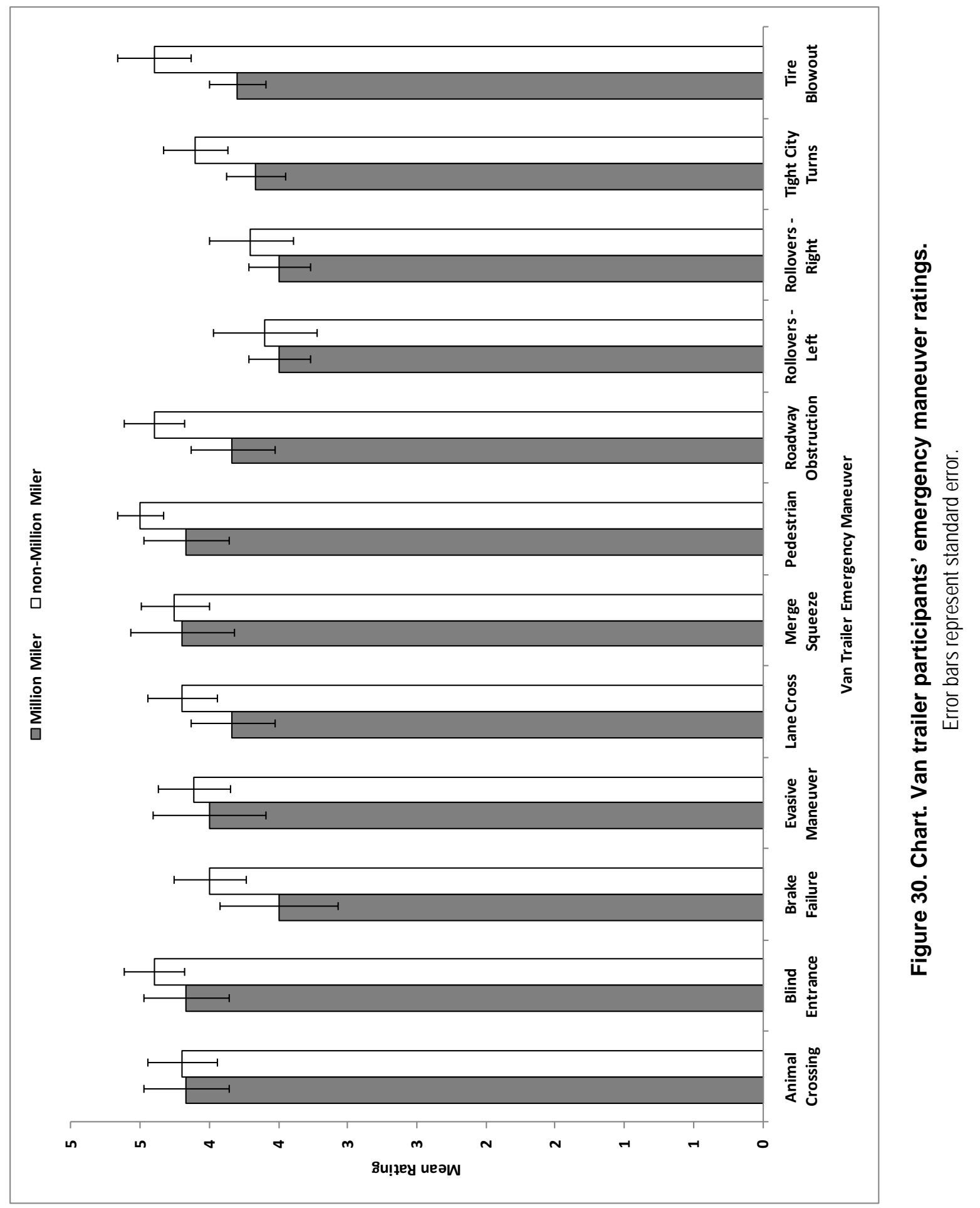




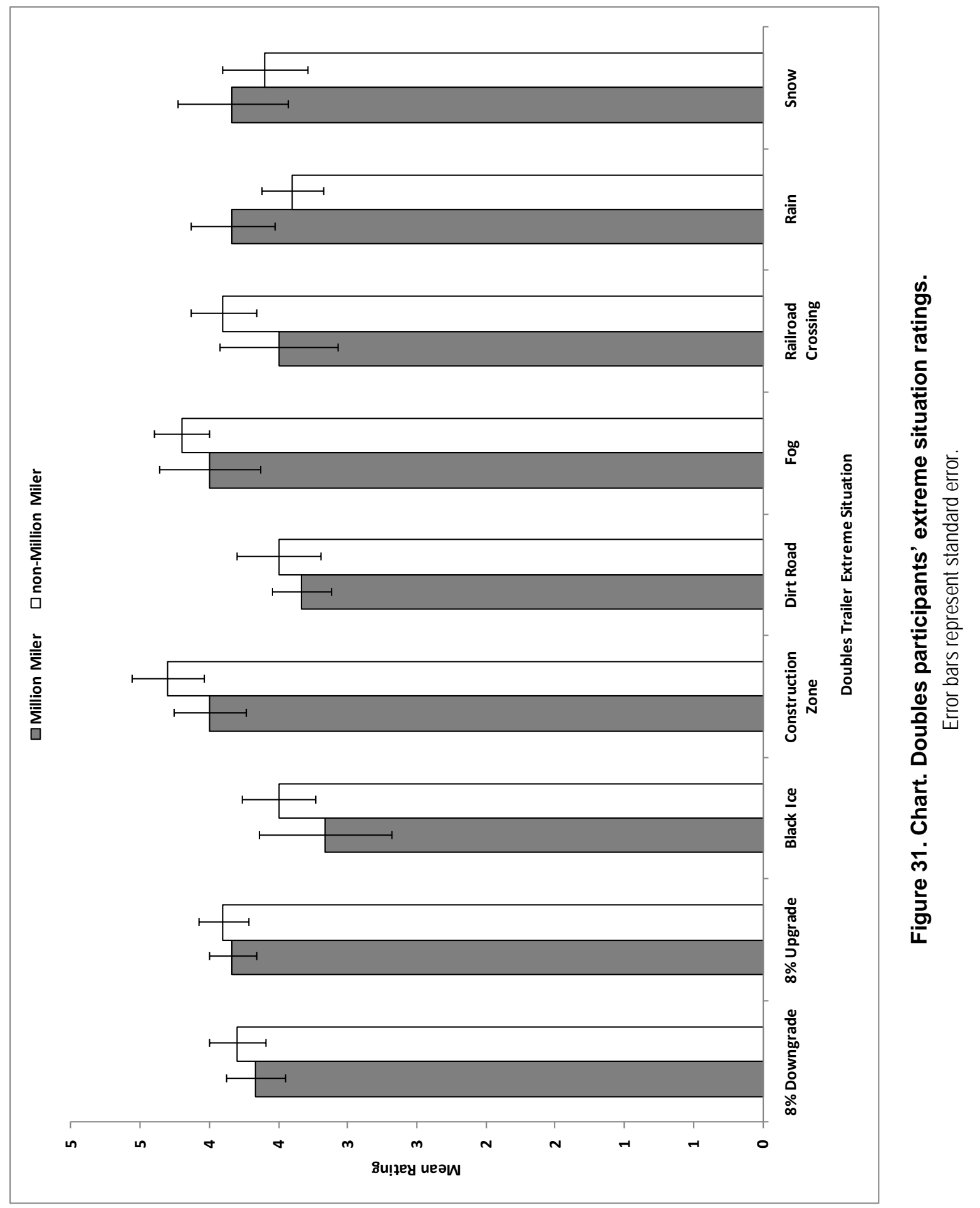




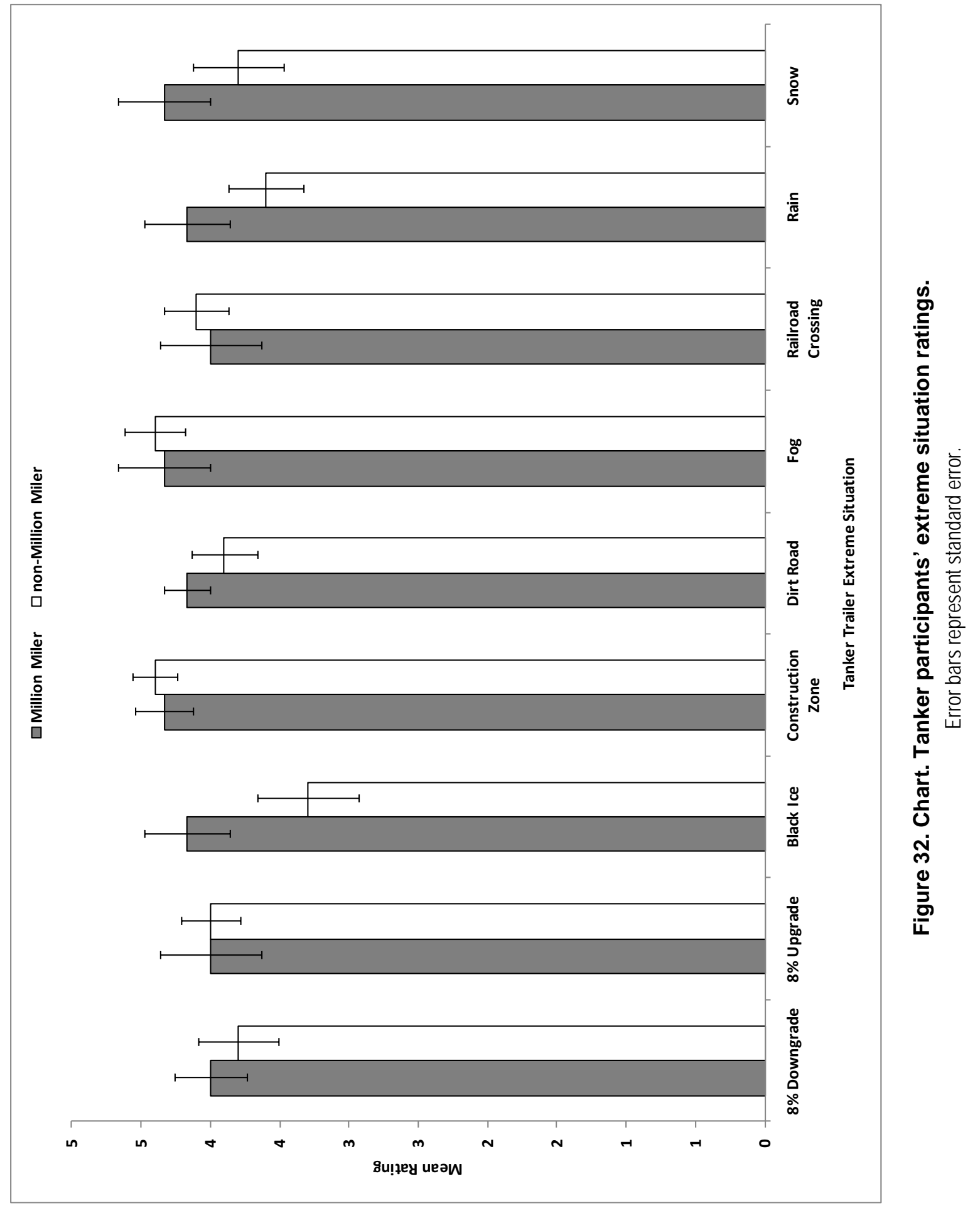




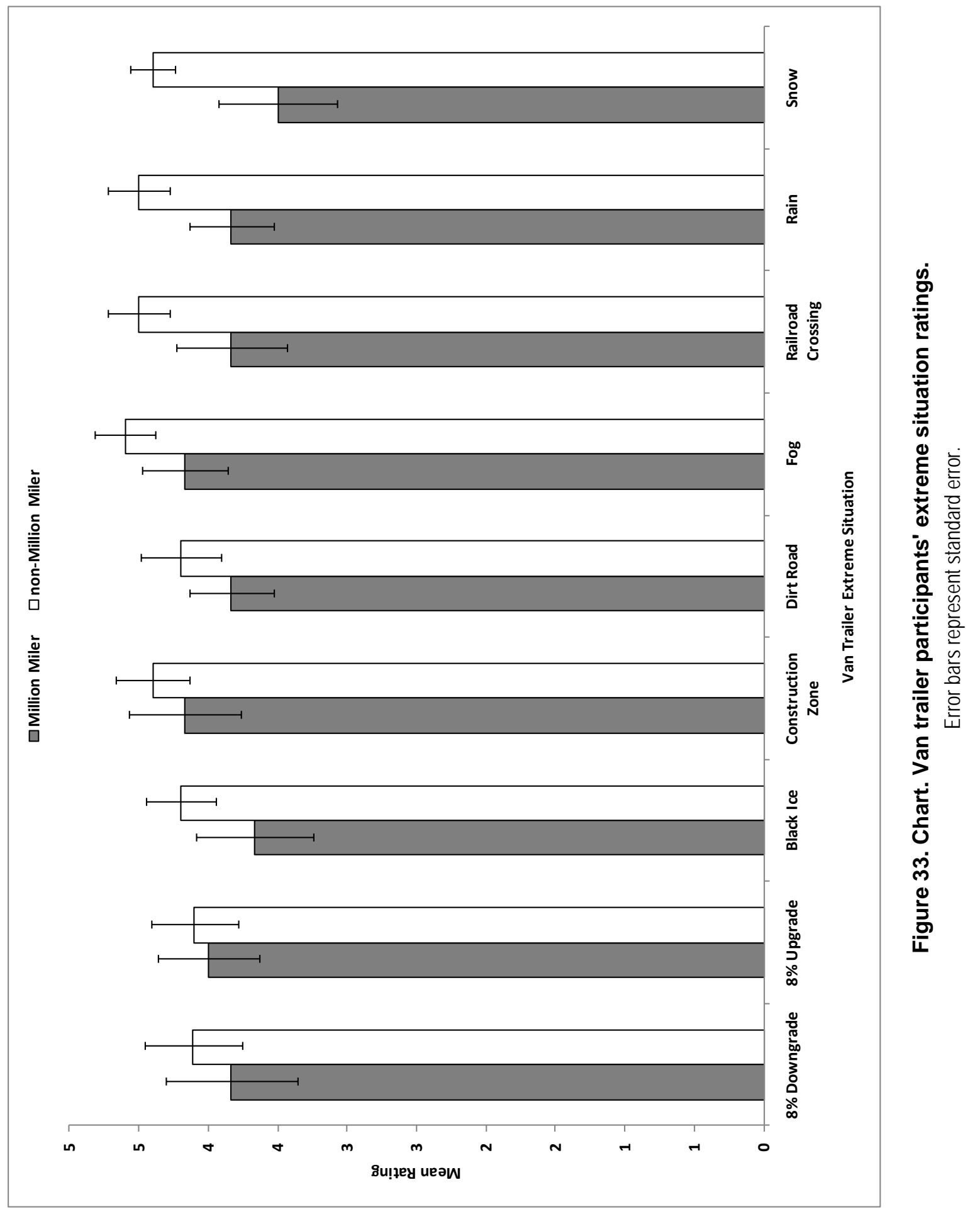

\& 


\subsubsection{Discussion}

Following the completion of the scenarios, both million-mile and non-million-mile participants across all three trailer configurations provided feedback on the usefulness and realism of the simulator, as well as feedback on which emergency maneuvers and extreme events were most realistic. These participants completed 12 emergency maneuvers and 9 extreme condition scenarios. No overall pattern of statistically significant differences between million milers and non-million milers were found in the ratings of the showcase scenarios' realism. When participants' ratings for the emergency maneuvers and extreme conditions were evaluated, the majority of participants felt these showcase scenarios were realistic when compared to their real world counterparts. However, the black ice extreme condition proved difficult to replicate in the simulated environment; thus, several realistic driver cues in likely black ice conditions were missing from this scenario.

Both million milers and non-million milers in the doubles trailers and tanker trailer rated safety and safe operations in the top three most effective aspects of the simulator. This is an important finding, as these participants indicated that both the safety and safe operations of a CMV driving simulator can provide beneficial training and experience for these emergency maneuvers and extreme conditions. Another important aspect of the simulator was vehicle dynamics, especially in relation to the vehicle dynamics of tanker trailers and doubles trailers. Here, simulation provided a safe alternative in exposing drivers to emergency maneuvers, in comparison to the risk of a crash associated with replicating these emergency maneuvers in a real tractor-trailer. Both million-mile and non-million-mile van trailer participants rated safety or safe operations as one of the top three aspects of a truck simulator. However, neither million milers nor non-million milers rated both aspects of safety and safe operations together in the top three. This may be explained by the fact that van trailers do not present the same vehicle dynamics challenges in the other trailer configurations. Thus, participants who pull van trailers may feel that safety and safe operations provided by the simulator are factors that are not as critical when training and practicing on a simulator.

Participants were also asked to provide open-ended comments. Some of the most notable comments from participants were to add night driving, high bridges with wind gusts, more city driving, and provide a better replication of the sound of a truck engine.

These findings indicated the simulator was able to produce a realistic simulation of many different types of emergency maneuvers and extreme conditions. Most drivers provided favorable ratings for the ability of the simulator to replicate potentially hazardous situations in a safe manner; thus, they believed the simulator could be used successfully to train drivers in defensive driving skills. Similarly, the emergency maneuver and extreme condition scenarios were deemed to be generally realistic by participants with few differences between million milers and non-million milers in ratings. This suggests the realism in the simulated events encountered were not limited to a certain group of drivers based on the amount of driving experience. 
[This page intentionally left blank.] 


\section{COST EFFECTIVENESS ANALYSIS OF ENTRY-LEVEL CMV DRIVER TRAINING}

\subsection{INTRODUCTION}

This section presents an analysis of the costs associated with each of the four types of training (i.e., conventional, simulator, informal, and CDL-focused) examined in the course of this study. This is a key analysis, as there is increasing interest in the training of commercial vehicle drivers. The first section of this chapter presents the computation of specific cost components that are common to two or more of the training methods. Following this, is a description of the safety metrics that the research team investigated as part of the analysis to compute the benefits, as well as a comparison of the total costs incurred for each training method. Note that, unless stated otherwise, all the costs presented in this analysis are computed in 2008 U.S. dollars. The findings presented herein provide useful information in determining the optimal method of training entrylevel CMV drivers.

According to the Office of Management and Budget (1992), benefit-cost analysis (BCA) is the recommended technique to use in a formal economic analysis in government programs or projects. However, the lack of quantifiable safety benefits data precluded this form of analysis. Cost-effectiveness analysis is a less comprehensive technique, but can be appropriate when the benefits from competing alternatives cannot be included within the model. While a BCA is a useful tool selecting among alternative training methods, the use of this technique in the present study is complicated by difficulties in identifying and monitoring training benefits, isolating the influence of training on safety performance measures, incorporating intangible or indirect benefits, and assigning monetary value to each aspect of the training.

\subsection{COSTS ASSOCIATED WITH TRAINING}

This section presents a description and computation of costs of common elements associated to the different training methods including trainee's and trainer's time, truck and simulator use and classroom facility and simulator facilities (i.e., direct training costs) and other (i.e., indirect) training costs.

\subsubsection{Direct Training Costs}

\subsubsection{Trainee's time}

The trainee's time is computed as the time he/she expends in the totality of all training, using a salary comparable to the entry-level trainee. The National Occupational Employment and Wage Survey (Bureau of Labor Statistics, 2008) was used to estimate trainee salaries. The 25th percentile wage was used, and resulted in a best estimation for the entry-level driver earning \$14.21 per hour. However, FMCSA recommends adding 31 percent to cover the fringe benefits (Murray, Shackelford, \& Houser, 2009), resulting in a total per hour wage of \$18.61. Table 40 displays the breakdown of training hours. 
Table 40. Number of Trainee Hours in Each Training Program

\begin{tabular}{|l|r|r|r|r|r|r|r|}
\hline $\begin{array}{c}\text { Training } \\
\text { Group }\end{array}$ & $\begin{array}{c}\text { Class } \\
\text { Time }\end{array}$ & $\begin{array}{c}\text { BTW } \\
\text { Range }\end{array}$ & $\begin{array}{c}\text { BTW } \\
\text { Road }\end{array}$ & $\begin{array}{c}\text { Simulator } \\
\text { Road }\end{array}$ & $\begin{array}{c}\text { Simulator } \\
\text { Range }\end{array}$ & $\begin{array}{c}\text { Observation } \\
\text { Time }\end{array}$ & Total Number \\
\hline Conventional & 147.25 & 24.5 & 26.25 & N/A & N/A & 100 & 300 \\
\hline Simulator & 147.25 & 9.5 & 10.25 & 16.0 & 15.0 & 100 & 300 \\
\hline Informal & Varies & Varies & Varies & Varies & Varies & Varies & Varies $6-160^{\star}$ \\
\hline CDL-focused & $24-70 \dagger$ & $160-210 \dagger$ & Varies & N/A & N/A & N/A & $160-320^{\star}$ \\
\hline
\end{tabular}

* Based on responses from the participants

$\dagger$ Based on information from the schools attended by the participant. BTW Range and Road hours include observation time

\subsubsection{Trainer or Instructor Time}

The trainer's time was computed as the time expended by the trainer in the totality of training hours, divided by the number of trainees (20 students per trainer for the class instruction and 3 students per trainer for BTW and simulator modes). To compute the trainer salary, two sampled salaries representative of conventional CMV driver trainers (average of $\$ 58.35$ per hour) and the 75th percentile Driver Salary from the National Occupational Employment and Wage Survey (Bureau of Labor Statistics, 2008; \$29.55 per hour, including 31 percent to cover fringe benefits) were considered. The average of the three salaries ( $\$ 48.75$ per hour) was used as the best estimate for the salary of a trainer. Table 41 displays the breakdown of the trainer's time computed as the trainer's total time divided by the number of participants in each of the training sessions. 


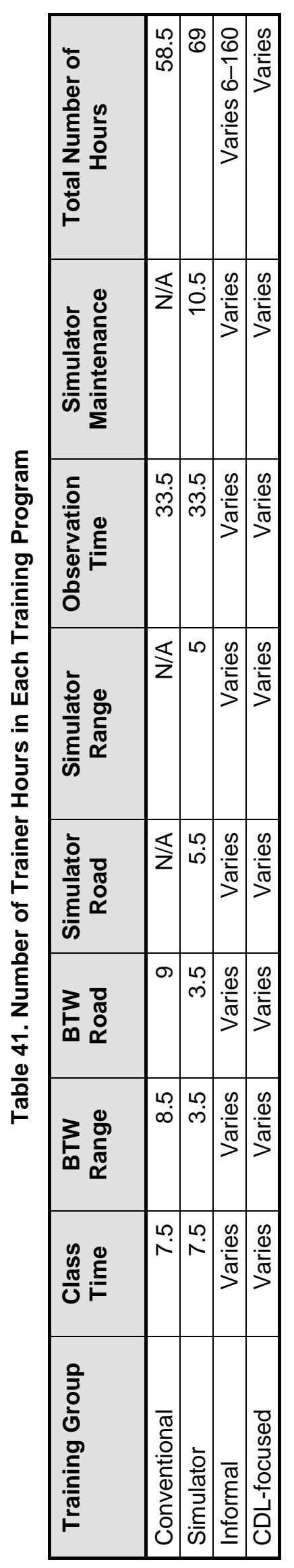




\subsubsection{Facilities}

Facilities costs included the construction, leasing, or sharing of facilities for the purpose of training. Two types of facilities were involved in truck driver training: a room to host classroom instruction (for the conventional, simulator, and CDL-focused training groups), as well as space to host the simulator (for the simulator training group). The facilities costs per participant depends on the number of training sessions per year, participants per training session, and facility location, as well as any potential space-sharing between CMV driver training and other purposes. For this analysis, the facilities (classrooms and simulator rooms) were treated as if they are dedicated for training purposes. Overall, the average cost for leasing is a minor cost component in the total cost of training (regardless of any space-sharing).

It was assumed that 900 square feet and 500 square feet were needed for the classroom and simulator facilities, respectively. Lease cost varies substantially by location and facility and, for this study, an average rental cost of $\$ 10 / \mathrm{ft}^{2}$ per year was used. This estimate was based on reported average lease costs for the third quarter of $2009\left(\$ 20.94 / \mathrm{ft}^{2}\right.$ for office space and $\$ 6.36 / \mathrm{ft}^{2}$ for industrial building space; Willis, 2009). The total facility cost per student per hour was computed, assuming a total of 260 training days in a year, with the facility total utilization at either 50 percent (in the case of the instruction room) or 80 percent (in the case of the simulator room) for a possible 8 hours per day. The total number of available hours was multiplied by the average number of participants (20 trainees for the classroom, 3 for the simulator room) for a total of facilities-student-hours per year. The total cost of the room per year (including maintenance costs) is divided by the total number of student hours per year to obtain a cost/hour/student to use each of the facilities. A listing of facilities and equipment costs used are provided in Table 42.

Table 42. Facilities and Equipment Costs

\begin{tabular}{|l|c|c|}
\hline \multicolumn{1}{|c|}{ Category } & Classroom & Simulator Room \\
\hline Facility cost per year $(\$)$ & $\$ 9,000$ & $\$ 5,000$ \\
\hline Maintenance Cost per year (\$) & $\$ 900$ & 500 \\
\hline Percentage Use & 50 & 80 \\
\hline Possible Number of Training Days & 260 & 260 \\
\hline Effective Number of Training Days & 130 & 208 \\
\hline Number of hours per day & 8 & 8 \\
\hline Number of hours per year & 1,040 & 1,664 \\
\hline Number of students per room & 20 & 3 \\
\hline Total Number of student Hours per Year & 20,800 & 4,992 \\
\hline Total Cost Per Year $(\$)$ & $\$ 9,900$ & $\$ 5,500$ \\
\hline Cost per student per hour (\$) & $\$ 0.48$ & $\$ 1.10$ \\
\hline
\end{tabular}

\subsubsection{Equipment}

Training equipment (e.g., trucks, trailers, simulators) is typically the property of the training organization. However, as truck driver training involves a substantial number of hours of BTW time for both trainers and trainees, it is unreasonable to assume that a truck could be easily shared between a CMV driver training program and other purposes. Thus, the trucks and the 
simulator were assumed to be dedicated for CMV driver training. If this equipment is used for other purposes, the cost of that training method will decrease in each unique scenario.

The cost of the equipment can be amortized over its useful life, with yearly maintenance costs and operational costs added to find the annual cost. In order to use the same unit costs for the different training methods, the cost per student per hour was computed. The number of training days per year was assumed to be 260 and, assuming an 8 hour day, the total number of hours in a year that the equipment (truck or simulator) was in use for training was determined. The result was multiplied by the number of students able to be trained each hour of operation (e.g., three students per truck) to compute the total number of student hours per year. Finally, the annual cost was divided by the total number of student hours per year to obtain the cost per hour per student.

\subsubsection{Truck Use}

The cost of the truck was set at $\$ 100,000$ (based on the cost of a 2010 International ProStar twin drive-axle tractor with a sleeper cab; this is a vehicle similar to the ones used to train the conventional group participants), with a useful life of 10 years. The cost of the truck was amortized over the truck's useful life. The operational costs included the vehicle operational costs and the driver costs. For this particular study the driver cost was included as part of the trainee costs and not included as equipment cost. The operational cost of the vehicle itself includes vehicle purchase/lease, maintenance, fuel/oil, tires, and insurance costs. A recent analysis of operational costs was used to calculate the operational costs of a truck on a per mile basis (American Transportation Research Institute, 2008). Table 43 provides these values.

Table 43. Operational Costs Considered to Compute the Average Cost per Mile for Trucks

\begin{tabular}{|l|c|c|}
\hline \multicolumn{1}{|c|}{ Motor Carrier Marginal Expenses } & Cost per Mile (\$) & Percent Cost per Mile \\
\hline Fuel-Oil Cost & $\$ 0.634$ & $72.21 \%$ \\
\hline Repair and Maintenance & $\$ 0.092$ & $10.48 \%$ \\
\hline Fuel Taxes & $\$ 0.062$ & $7.06 \%$ \\
\hline Truck Insurance Premium & $\$ 0.06$ & $6.83 \%$ \\
\hline Tires & $\$ 0.03$ & $3.42 \%$ \\
\hline Total & $\$ 0.878$ & $100 \%$ \\
\hline
\end{tabular}

Table 44 shows the cost per truck per student per hour, based on 80 percent utilization, for a total of 208 days, 8 hours per day, and an average of 200 miles per day. The cost for using the truck was $\$ 9.32 /$ hour/student. It is important to notice that this cost assumed three students to be present in the truck at any time, so the cost of the truck is $\$ 27.96 /$ hour. 
Table 44. Costs Associated with Truck Use

\begin{tabular}{|l|c|}
\hline \multicolumn{1}{|c|}{ Truck Aspect } & Amount \\
\hline Initial cost (\$) & $\$ 100,000$ \\
\hline Service life & 10 years \\
\hline Initial cost/service life & $\$ 10,000$ \\
\hline Operation cost/mile & $\$ 0.88$ \\
\hline Number of miles per day & 200 \\
\hline Percentage use & 80 \\
\hline Number of possible training days per year & 260 \\
\hline Number of training days per year & 208 \\
\hline Number of hours per training day & 8 \\
\hline Number of hours per year & 1,664 \\
\hline Number of miles per year & 41,600 \\
\hline Total operational cost per year & $\$ 36,525$ \\
\hline Total cost per truck per year & $\$ 46,525$ \\
\hline Number of students per truck & 3 \\
\hline Total number of student hours & 4,992 \\
\hline Cost per student per hour & $\$ 9.32$ \\
\hline
\end{tabular}

\subsubsection{Simulator Use}

For the purposes of this analysis, a simulator with a fixed cost of $\$ 290,000$ was used (based on a fourth quarter 2009 sales quote by the simulator manufacturer). The useful life of the simulator was determined to be 15 years, and the cost was amortized over the simulator's useful life. Similar to trucks, operating costs are associated with the simulators, mainly maintenance and repair costs, usually covered under warranty agreements and personnel costs. A basic warranty is included with most purchases and a full extended hardware warranty covering all parts and labor may be purchased. The operating costs incurred in this study (including the replacement of two projector bulbs, three video cards, a broken shift handle, a transmission, and a broken turn signal switch) were used form these estimates.

While the simulator cost noted above $(\$ 290,000)$ represents the cost of the training simulator as used in the present study (not including the doubles and tank trailer models used in the ACS), it is possible that other configurations of simulators with lower initial acquisition costs may be used. In addition to the configuration used, a lower priced FAAC TT-2000-V5 +3 DOF simulator (three forward visual channels and two rear video channels, not including tank trailer or doubles trailers) was included. This configuration has a manufacturer-quoted cost of $\$ 260,000$. It should be noted that the quoted cost of $3 \mathrm{DOF}$ is $\$ 40,000$. Finally, based on an informal query of carriers and simulator manufacturers, it was determined that an L3 TransSim VS IV is in common use within the industry. A sales quote of $\$ 125,000$ was obtained for this configuration. The cost of all three configurations of truck simulators are presented in Table 45. Further information on the types of simulators identified as in use within the industry, as well as their features and prices, are provided in appendix $\mathrm{M}$.

Personnel costs include the costs of a person conducting the training and controlling the simulator. However, for this analysis, both are considered as trainee costs and are not included in 
simulator use costs. The cost of the simulator varies with the number of participants per room (as is shown in table 45 ) for a total of $\$ 5.07, \$ 4.21$, and $\$ 3.37$ per student per hour (assuming three students per class) for the FAAC (study) simulator, mid-level FAAC simulator, and TransSim VS IV simulator, respectively.

Table 45. Costs Associated with Simulator Use

\begin{tabular}{|l|c|c|c|}
\hline \multicolumn{1}{|c|}{ Simulator Aspect } & $\begin{array}{c}\text { Cost of FAAC } \\
\text { Simulator Used } \\
\text { in Study }\end{array}$ & $\begin{array}{c}\text { Cost of Mid- } \\
\text { Level FAAC } \\
\text { Simulator }\end{array}$ & $\begin{array}{c}\text { Cost of L3 TransSim } \\
\text { VS IV }\end{array}$ \\
\hline Initial Cost (\$) & $\$ 290,000$ & $\$ 260,000$ & $\$ 125,000$ \\
\hline Service Life (years) & 15 & 15 & 15 \\
\hline Initial Cost/Service Life & $\$ 19,333$ & $\$ 17,333$ & $\$ 8,333$ \\
\hline Maintenance Cost per year (\$) & $\$ 6,000$ & $\$ 6,000$ & $\$ 6,000$ \\
\hline Percentage Use & $80 \%$ & 80 & 80 \\
\hline Number of Training Days per Year & 260 & 260 & 260 \\
\hline Effective Number of Training Days & 208 & 208 & 208 \\
\hline Number of hours per day & 8 & 8 & 8 \\
\hline Number of hours per year & 1,664 & 1664 & 1664 \\
\hline Number of students per simulator & 3 & 3 & 3 \\
\hline Total Number of student hours per Year & 4,992 & 4992 & 4992 \\
\hline Total Cost Per Year $(\$)$ & $\$ 25,333 /$ year & $\$ 23,333$ & $\$ 14,333$ \\
\hline Cost per student per hour $(\$)$ & $\$ 5.61$ & $\$ 4.67$ & $\$ 2.87$ \\
\hline
\end{tabular}

\subsubsection{Training Material Development}

Typically, developing training materials has two associated components: a onetime cost for developing the materials and the recurring cost for updating the materials. Not only was the cost of developing training materials similar for all groups (with the exception of the informal group), but the costs were also assumed to be lower due to the high number of trainees. For example, for $\$ 10,000$ spent on the development of training materials with a useful life of 5 years, over 10 courses (with 20 participants each) the additional cost of training material development is \$10 per student. Therefore, training material development costs were not taken into account in the analysis.

\subsubsection{Indirect Training Costs}

\subsubsection{CDL Pass Rate}

The most used standard reported for the measure of training effectiveness is CDL pass rate (Brock, McFann, Inderbitzen, \& Bergoffen, 2007). A pass rate greater than 70 percent is considered successful. All training methods examined in the present study had a pass rate greater than 70 percent (see table 46). In addition to the cost of taking the test, there was an opportunity cost of beginning employment as a licensed driver. For this particular study, it was assumed that the time between tests (assuming the prior attempt at the test was unsuccessful) was 2 weeks. These 2 weeks resulted in a reduction in salary (a cost for the training method) of $\$ 1,489$. 
Table 46. CDL Test Pass Rate for Study Drivers and Cost

\begin{tabular}{|l|r|r|r|r|r|r|}
\hline \multicolumn{1}{|c|}{ Group } & $\begin{array}{c}\text { \# Test } \\
\text { Scores }\end{array}$ & $\begin{array}{c}\text { First } \\
\text { Attempt } \\
\text { Road }\end{array}$ & $\begin{array}{c}\text { First Attempt } \\
\text { on the } \\
\text { Range }\end{array}$ & $\begin{array}{c}\text { Second } \\
\text { Attempt on } \\
\text { the Range }\end{array}$ & $\begin{array}{c}\text { Third } \\
\text { Attempt on } \\
\text { the Range }\end{array}$ & $\begin{array}{c}\text { Total Cost } \\
\text { per } \\
\text { Participant }\end{array}$ \\
\hline Conventional & 33 & $100 \%$ & $100 \%$ & $0 \%$ & $0 \%$ & $\$ 0$ \\
\hline Simulator & 32 & $100 \%$ & $91 \%$ & $6 \%$ & $3 \%$ & $\$ 186$ \\
\hline Informal & 9 & $100 \%$ & $78 \%$ & $11 \%$ & $11 \%$ & $\$ 496$ \\
\hline CDL-focused & 23 & $100 \%$ & $78 \%$ & $22 \%$ & $0 \%$ & $\$ 324$ \\
\hline
\end{tabular}

\subsubsection{Employment}

Another possible measure of success is the number of driver-graduates hired for driving positions (Brock et al., 2007). The number of participants currently employed as Class A CDL drivers is shown in Table 47. The potential lost income is substantial. However, it is not clear if some participants are working in a different category of employment or if they were not actively seeking employment as a Class A CDL driver. Therefore, this variable was not included in the analysis.

Table 47. Study Driver Employment Status and Cost

\begin{tabular}{|l|c|c|c|c|c|}
\hline Training Group & $\begin{array}{c}\text { Total \# } \\
\text { Participants }\end{array}$ & $\begin{array}{c}\text { Total \# Participants } \\
\text { with a Job }\end{array}$ & $\begin{array}{c}\text { \% With a } \\
\text { Job }\end{array}$ & $\begin{array}{c}\text { \% Without a } \\
\text { Job }\end{array}$ & $\begin{array}{c}\text { Potential } \\
\text { Cost (\$) }\end{array}$ \\
\hline Conventional & 33 & 10 & 30 & 70 & $\$ 26,979$ \\
\hline Simulator & 32 & 15 & 47 & 53 & $\$ 20,564$ \\
\hline Informal & 9 & 9 & 100 & 0 & $\$ 0$ \\
\hline CDL-focused & 33 & 8 & 24 & 76 & $\$ 29,325$ \\
\hline
\end{tabular}

\subsection{TOTAL COST FOR DIFFERENT TRAINING GROUPS}

\subsubsection{Conventional Training}

Conventional training occurred in both classroom and BTW settings. After receiving instruction on concepts and techniques, entry-level drivers were allowed time to practice maneuvers and respond to situations while driving actual trucks under the supervision of trainers. In order to obtain PTDI certification, these courses must include a minimum of 104 hours of classroom time and 44 hours BTW training per student (using 60-minute hours; PTDI, 1999). The direct and total costs of this type of training are shown in Table 48 and Table 49 respectively. 
Table 48. Total Direct Cost of Conventional Group Training

\begin{tabular}{|c|c|c|c|c|c|}
\hline Cost Category & Class & $\begin{array}{c}\text { BTW } \\
\text { Range }\end{array}$ & BTW Road & $\begin{array}{c}\text { Observation } \\
\text { Time }\end{array}$ & Total \\
\hline Trainee Hours & 147.3 & 24.5 & 26.3 & 100.0 & - \\
\hline Cost/hour & $\$ 18.60$ & $\$ 18.60$ & $\$ 18.60$ & $\$ 18.60$ & - \\
\hline Sub total & $\$ 2,740$ & $\$ 456$ & $\$ 489$ & $\$ 1,861$ & $\$ 5,546$ \\
\hline Trainer Hours & 7.36 & 8.17 & 8.75 & 33.50 & - \\
\hline Cost/hour & $\$ 48.80$ & $\$ 48.80$ & $\$ 48.80$ & $\$ 48.80$ & - \\
\hline Sub total & $\$ 359$ & $\$ 398$ & $\$ 427$ & $\$ 1,633$ & $\$ 2,817$ \\
\hline Facility Hours & 147.25 & - & - & - & - \\
\hline Cost/hour & $\$ 0.48$ & - & - & - & - \\
\hline Sub total & $\$ 70$ & - & - & - & $\$ 70$ \\
\hline Truck Hours & - & 24.5 & 26.3 & 100.0 & - \\
\hline Cost/hour & - & $\$ 9.30$ & $\$ 9.30$ & $\$ 9.30$ & - \\
\hline Sub total & - & $\$ 228$ & $\$ 245$ & $\$ 932$ & $\$ 1,405$ \\
\hline Subtotal Trainee & $\$ 2,740$ & $\$ 456$ & $\$ 489$ & $\$ 1,861$ & $\$ 5,546$ \\
\hline Subtotal Training & $\$ 429$ & $\$ 626$ & $\$ 671$ & $\$ 2,565$ & $\$ 4,292$ \\
\hline Total & $\$ 3,169$ & $\$ 1,082$ & $\$ 1,160$ & $\$ 4,426$ & $\$ 9,838$ \\
\hline
\end{tabular}

Table 49. Total Costs for Conventional Training

\begin{tabular}{|l|r|}
\hline \multicolumn{1}{|c|}{ Training Component } & \multicolumn{1}{c|}{ Cost } \\
\hline Training Costs & $\$ 9,838$ \\
\hline Cost Associated with not Passing the CDL Exam & $\$ 0$ \\
\hline Total Cost & $\$ 9,838$ \\
\hline
\end{tabular}

\subsubsection{Simulator Training}

Participants in the simulator group received full-curriculum, entry-level training, similar to the conventional group. These participants were students at the DTCC CTP and received 19.75 hours of their practice drive time in a real tractor-trailer and 31 hours in a simulator. The direct costs of this type of training are shown in Table 50 (representing the simulator configuration used in the present study), Table 51 (representing a simpler, mid-level, configuration of the study simulator), and Table 52 (representing a L3 TransSim VS IV). Total costs for simulator training are presented in Table 53. The values for the other two simulators used for cost analysis were chosen from an informal questioning of the industry (appendix M). It should also be noted that this analysis does not include different estimations of the variations in electrical costs associated with simulator use, due to the need for additional ventilation and cooling capacity or energy demand of the simulator. 


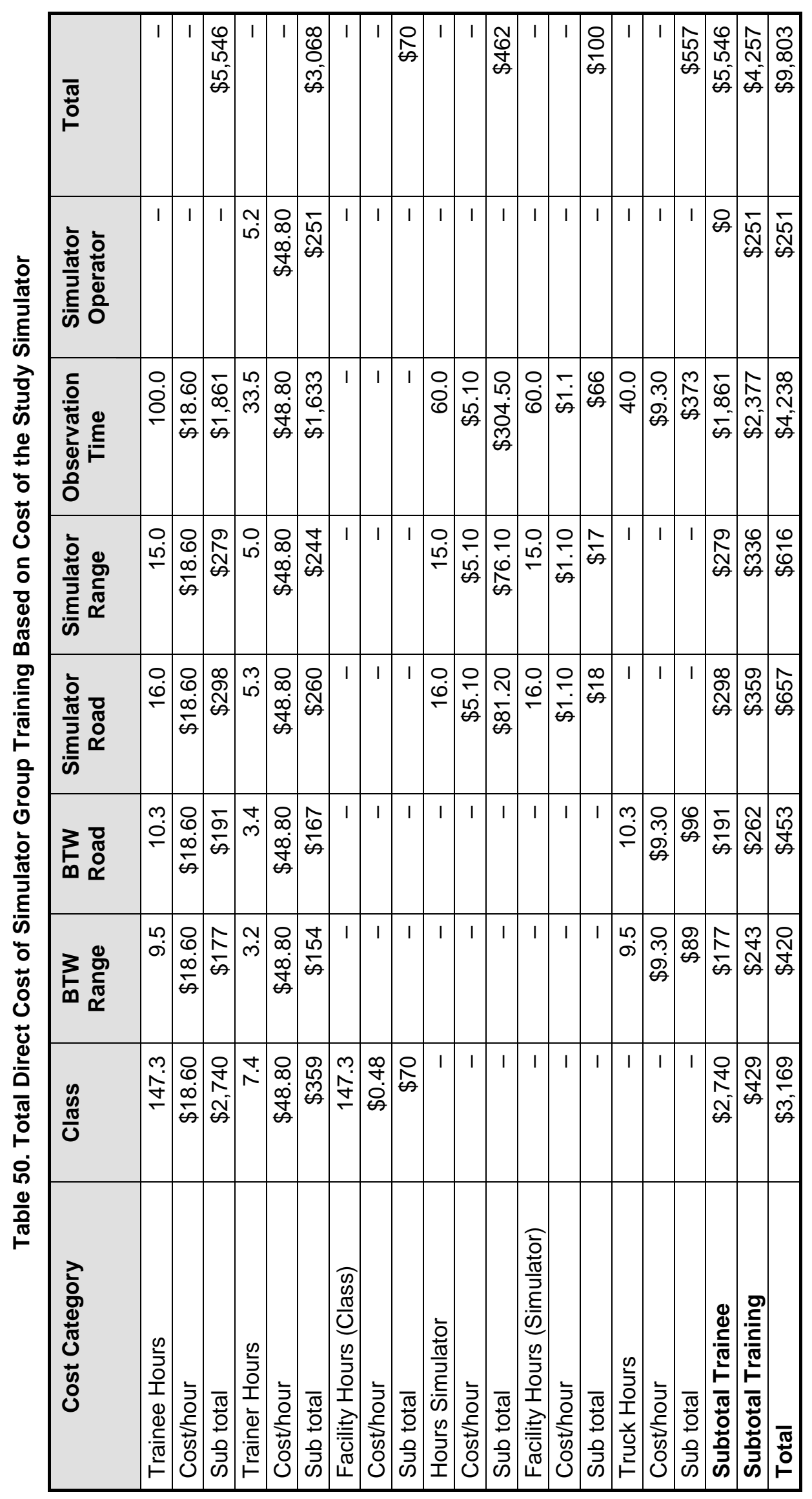




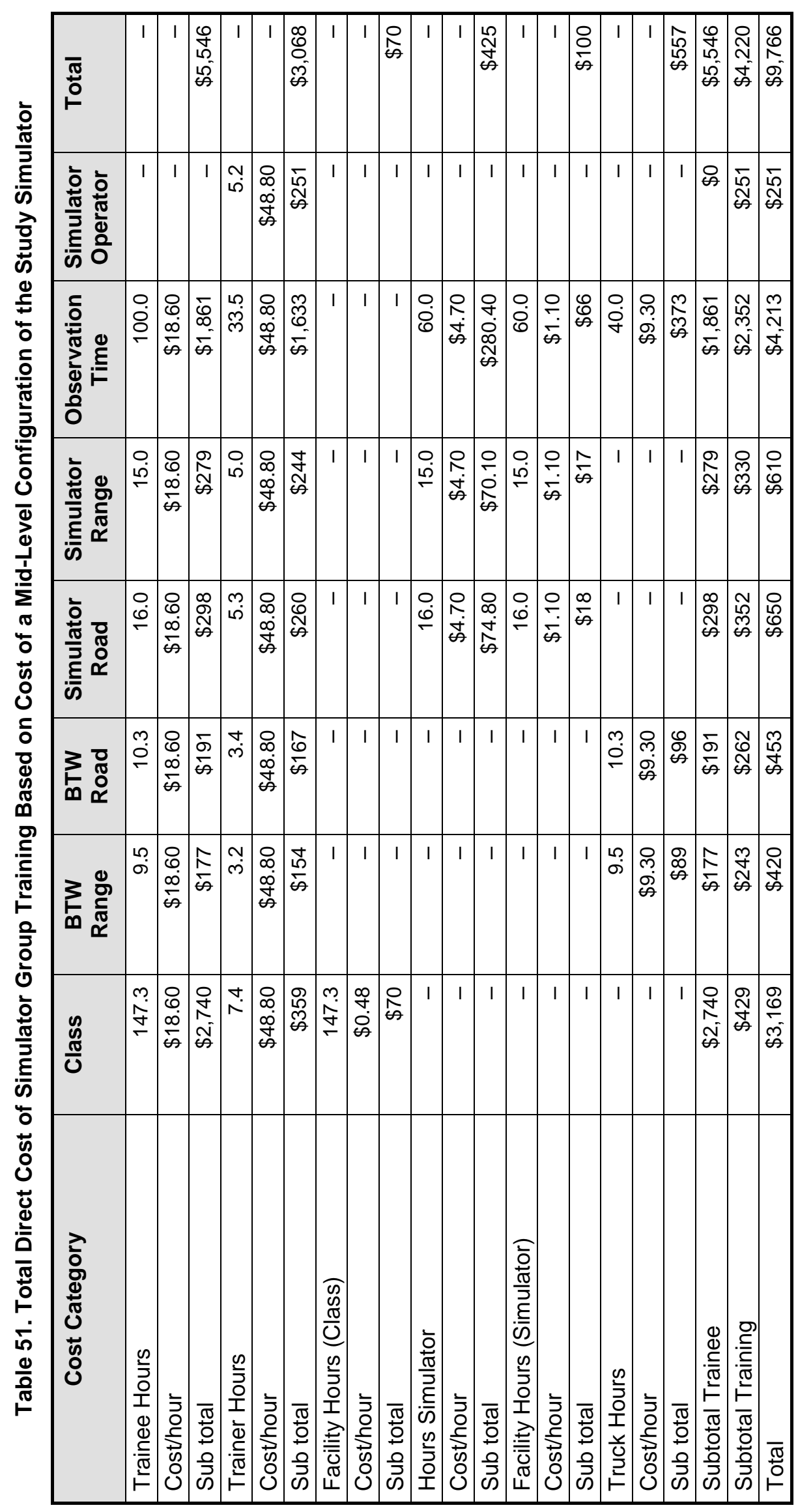




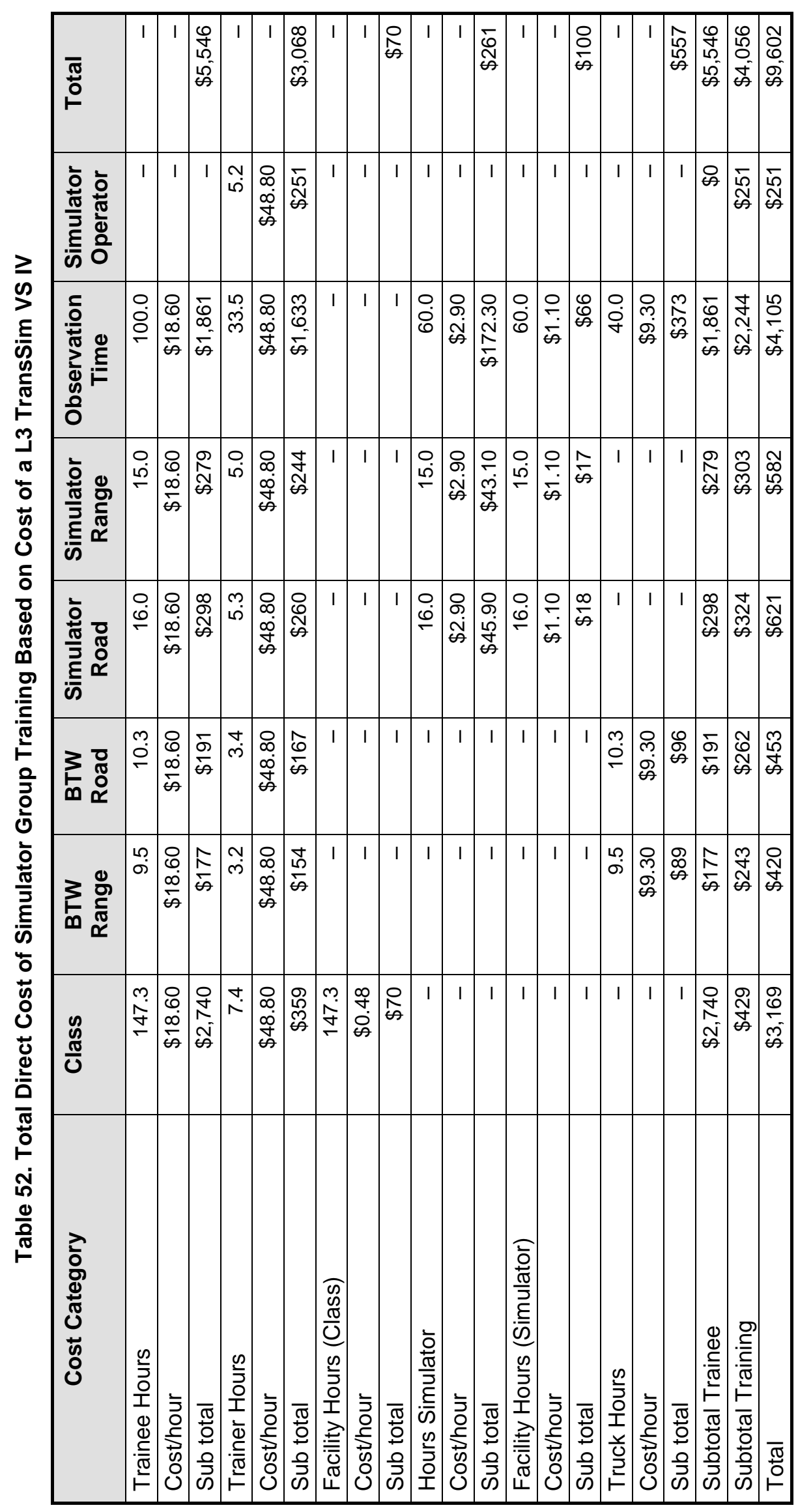


Table 53. Total Costs for Simulator Training

\begin{tabular}{|l|r|r|r|}
\hline \multicolumn{1}{|c|}{ Training Component } & \multicolumn{1}{|c|}{$\begin{array}{c}\text { FAAC } \\
\text { Simulator } \\
\text { Used in Study }\end{array}$} & $\begin{array}{c}\text { Mid-Level } \\
\text { FAAC } \\
\text { Simulator }\end{array}$ & $\begin{array}{c}\text { L3 TransSim } \\
\text { VS IV }\end{array}$ \\
\hline Training Costs & $\$ 9,803$ & $\$ 9,766$ & $\$ 9,602$ \\
\hline Cost Associated with not Passing the CDL Exam & $\$ 186$ & $\$ 186$ & $\$ 186$ \\
\hline Total Cost & $\$ 9,989$ & $\$ 9,952$ & $\$ 9,788$ \\
\hline
\end{tabular}

\subsubsection{Informal Training}

Informal training occurred when a driver received training in an informal or non-structured setting. No requirements for classroom or BTW time were present and the trainer may not be certified. Informal training may occur in situations such as an entry-level driver receiving training from a friend, family member, or coworker. Participants for the informal training group were asked to complete a questionnaire regarding how they learned to drive a truck. Three drivers reported learning on the job, and six reported learning from a friend or family member.

Based on the information available regarding equipment costs, as well as informal group participants' estimates of their own training times, the number of hours and miles were used to compute the associated cost for this type of training. To calculate the cost of the truck usage, only the cost per mile was used (assuming the truck was used for other purposes). The trainee time was computed as the number of hours that the participant indicated, multiplied by $\$ 18.61$. One-on-one instruction was assumed, so the trainer's time was equal to the trainee's time. The median cost of this type of training was $\$ 988$. In addition, the costs associated with not passing the CDL exam in the first attempt is $\$ 496$ per participant for a total cost of \$1,484 per participant (Table 54).

Table 54. Costs for Informal Training

\begin{tabular}{|l|r|}
\hline \multicolumn{1}{|c|}{ Training Component } & \multicolumn{1}{c|}{ Cost } \\
\hline Training Costs & $\$ 988$ \\
\hline Cost Associated with not Passing the CDL Exam & $\$ 496$ \\
\hline Total Cost & $\$ 1,484$ \\
\hline
\end{tabular}

\subsubsection{CDL-focused Training}

CDL-focused participants were those CDL test-takers who received training at a CDL-focused truck driver training school. CDL-focused training mimics many of the features of conventional training (including classroom and BTW training components), however on a temporally compressed schedule. CDL-focused training typically lasts two to four weeks and involves drivers learning the basic information needed to obtain a CDL instruction permit (learner's permit), followed by BTW training for specific vehicle skills. The participants in a CDL-focused program were asked to complete a questionnaire regarding what school they attended, how many training hours they experienced (both BTW and classroom), and how many trainees were in the course. This information is provided in Table 55. 
Table 55. CDL-focused Training Program Information

\begin{tabular}{|c|c|c|c|c|c|}
\hline Program & \# Participants & Class (hrs) & BTW/Range (hrs) & Total (hrs) & Cost \\
\hline 1 & 17 & 24 & 136 & 160 & $\$ 4,000$ \\
\hline 2 & 2 & 30 & 179 & 200 & $\$ 2,500$ \\
\hline 3 & 5 & 80 & 240 & 320 & $\$ 4,172$ \\
\hline 4 & 2 & N/A & - & - & - \\
\hline 5 & 1 & 69 & 138 & 207 & $\$ 3,700$ \\
\hline
\end{tabular}

Note: Program 3 requires participants to attend at least 80 percent of class hours. Program 5 has a duration of 3 weeks: 1 week for classroom instruction and 2 weeks for Range and BTW.

This was consistent with the cost reported on the FMCSA proposed regulations on minimum training requirements for entry-level CMV drivers (FMCSA, 2007). A prior FMCSA study included interviews of staff members of multiple training schools and organizations and determined that training prices varied widely among the different schools. However, the respondents replied that a cost of approximately $\$ 4,000$ per 4 weeks of training was typical. This translates into $\$ 1,000$ of direct training cost for 40 hours of training.

Participants reported that training hours vary, even in the same school. However, participants recalled receiving more classroom instruction and less BTW and range time than specified by the school. For some specific cases, the information was verified (e.g., one case of a participant receiving 1 day of training because the instructor considered the trainee ready to take the CDL test). In general, when the trainer determines that the participant is ready to take the exam, the student is encouraged to take it.

While the weighted average cost of the CDL-focused training program was $\$ 3,902$, the number of hours is higher than the 160 hours (with a weighted average of 190 hours, assuming 90 percent of the hours for program). Also, most participants indicated receiving BTW and range instruction with four or five fellow participants. This was a substantial difference with the simulator and conventional training groups, which have a ratio of three students per instructor (allowing $1 / 3$ for practice and 2/3 for observation time). The cost of the simulator or truck is significantly reduced as the number of participants is increased. It was assumed that the tuition cost covered all costs associated with training. The cost of the trainee time, at \$18.61 per 190 hours, was $\$ 3,549$. The costs associated with not passing the CDL exam in the first attempt was $\$ 324$ per participant. This, added to the training costs, resulted in a total cost of $\$ 7,775$ (Table $56)$.

Table 56. Tuition Costs, Trainees' Time, and Other Costs for CDL-focused Training

\begin{tabular}{|l|r|}
\hline \multicolumn{1}{|c|}{ Training Component } & Cost \\
\hline Tuition cost & $\$ 3,902$ \\
\hline Trainee's Time & $\$ 3,549$ \\
\hline Cost Associated with not Passing the CDL Exam & $\$ 324$ \\
\hline Total Cost & $\$ 7,775$ \\
\hline
\end{tabular}




\subsection{BENEFITS ASSOCIATED WITH TRAINING}

This section presents description of the safety metrics that the research team explored as part of the analysis. The expected number of crashes, violations, and other benefits were analyzed as possible safety metrics to compute the benefits for the BCA.

\subsubsection{Crashes}

As noted by Brock et al., (2007), the frequency of commercial vehicle crashes is the overarching indicator of commercial driver training effectiveness.

Crash information for study participants was obtained through motor carriers and the USDOT. However, as the probability of any individual driver experiencing a crash in a year is very small, none of the study drivers were involved in any carrier- or police-reported crashes during the study period. Additionally, no data were available on other, non-reportable, crashes occurring on private property. Thus, this form of comparison was impossible.

\subsubsection{Violations}

Several studies have attempted to identify relationships between driver-performance and CMV crashes. Hughes (2001) examined the relationship between average moving violations per driver, driver out-of-service rates, vehicle out-of-service rates, and carrier crash risk. The results indicated that serious driving violations were a strong predictor of crashes. Likewise, Lantz and Blevins (2001) analyzed driver traffic conviction data from CDLIS to help identify high safetyrisk motor carriers. Results indicated significant positive linear correlations between the carrier driver history and every safety variable examined, with the exception of the crashes per driver rate.

Additionally, there are models allowing for the prediction of future crash involvement based on prior driver history (American Transportation Research Institute, 2005). These models use reckless driving, improper turning and lane change, and right-of-way violations to predict the likelihood of a future crash and were considered for use in the present analysis. However, similar to crashes, violations are relatively rare events. Due to the small sample of drivers with violations, this method of analysis was precluded.

\subsubsection{Other Benefits}

Training effectiveness performance measures may be grouped into two general categories: objective (performance) and subjective measures. Quantifying the latter category is difficult; however, consideration of subjective performance is important when evaluating a training program.

To provide additional insight into the trainees' perceptions and opinions of their training method, a self-evaluation questionnaire was conducted at 4-5 months following employment (see discussion Research Question 3a). The results show that, in general, participants had a slightly above average mean self-assessment in their control and safety skills. As this result was present across all groups, no further analysis was conducted. Likewise, a supervisory evaluation was obtained from the trainees' supervisors at 4-5 months after employment (see discussion of 
Research Question 7a). However, a lack of employer participation prevented the inclusion of this information in the benefits model.

\subsection{COST-EFFECTIVENESS ANALYSIS}

Due to the lack of quantifiable data for a benefits model, a cost-effectiveness analysis was used to compare the cost of different training methods (

Table 57). Because all the costs are incurred in year 1, there was no need to select the analysis period or discount rate. Note that, for the purposes of this analysis, the cost associated with failing the CDL test is assumed to be the same for the other simulator configurations examined.

Table 57. Training Costs

\begin{tabular}{|l|r|r|r|}
\hline \multicolumn{1}{|c|}{ Training Group } & $\begin{array}{r}\text { Direct } \\
\text { Training Cost }\end{array}$ & CDL Test & Total Cost \\
\hline Conventional & $\$ 9,838$ & $\$ 0$ & $\$ 9,838$ \\
\hline $\begin{array}{l}\text { Simulator } \\
\text { (FAAC, As Used in Study) }\end{array}$ & $\$ 9,803$ & $\$ 186$ & $\$ 9,989$ \\
\hline $\begin{array}{l}\text { Simulator } \\
\text { (FAAC, Mid-Level Configuration) }\end{array}$ & $\$ 9,766$ & $\$ 186$ & $\$ 9,952$ \\
\hline $\begin{array}{l}\text { Simulator } \\
\text { (L3 TransSim VS IV) }\end{array}$ & $\$ 9,602$ & $\$ 186$ & $\$ 9,788$ \\
\hline Informal & $\$ 988$ & $\$ 496$ & $\$ 1,484$ \\
\hline CDL-focused & $\$ 7,451$ & $\$ 324$ & $\$ 7,775$ \\
\hline
\end{tabular}

\subsection{RESULTS}

\subsubsection{Cost of Simulator versus Conventional Training}

Using the cost-effectiveness analysis option, the cost of the conventional training method was $\$ 9,838$. The cost for the simulator training method was $\$ 9,803$ (i.e, for the training only; $\$ 9,989$ if the time taken to pass the CDL exam is considered). There is, at a maximum, 2 percent difference in costs with simulator training representing \$35 less cost per student as compared to conventional training; both costs are incurred the first year so the rate of return does not have an impact on the results. To compute the costs, several assumptions were made regarding the costs per hour for trainer and trainee, facilities, equipment and number of students per classroom. These costs can have variability, such as the estimation of the trainee salary's or the type of truck used for the training that exceeds the 2 percent range. Additionally, it is expected that advances in simulation technologies as well as increased utilization of CMV simulation-based training will lead to the cost of the simulator decreasing over time, in addition to increasing the number of students that can be trained, without any deterioration in the quality of the training.

When other configurations of simulators are examined, additional cost savings are realized as compared to conventional training. The mid-level configuration (lacking rear video channels, as well as models for tanker and doubles trailers) of the driving simulator used in the present study 
represented a direct cost savings of $\$ 62$ as compared to conventional training. A lower cost simulator represented a direct cost savings of $\$ 236$ as compared to conventional training. However, it is important to note that the training outcomes of these two additional simulator configurations were not examined in the present study. Therefore, conclusions about the equivalency of training between these three simulators cannot be drawn; these values are only presented to provide a more complete view of the cost associated with different types of simulators.

\subsubsection{Cost of Conventional versus CDL-focused and Informal Training}

The informal training group has the least direct costs of the three methods. However, there was a large variation in the training hours and truck training hours, ranging from 50-160 hours of truck driving practice and instruction. The difference in costs, \$7,775 in the CDL-focused group and $\$ 9,838$ in the conventional group, is due to the differences in trainer and trainee time, both major components for the cost of conventional group and CDL-focused group training. For every hour of training in those groups, there is an increase of \$18.61 per participant hour. The average number of hours per trainee was 190 hours and 300 hours for the CDL-focused and conventional groups, respectively. This difference represents a minimum of \$2,047 (110 hours at \$18.61 per hour) in the total cost.

\subsection{CONCLUSIONS}

The present study explored the benefits and costs associated with multiple methods of training. However, a lack of quantifiable data for inclusion in a benefits model precluded BCA. Therefore, a cost-effectiveness analysis was conducted. The results of this cost-effectiveness analysis show only a small difference between direct costs (not including the cost of not passing the CDL exam) of simulator and conventional training methods, with the Simulator training costing \$35 less per trainee than the conventional training. While other forms of the simulator (such as a midlevel configuration of the simulator used in the study and a commonly used L3 TransSim driving simulator) represented further cost savings for simulation-based training, the equivalency of these simulators as compared to the simulator used in the study was not assessed. The cost of simulation equipment may decrease over time, if scales of implementation increase and technology costs decrease. This will have an effect on the cost effectiveness analysis of simulation training versus other types of training. In comparing conventional to CDL-focused and informal training, informal training was the least expensive of the three training methods. However, there were large variations in the training time reported by the drivers in this group. Trainee time was a major component for the cost of conventional and CDL-focused group training. 
[This page intentionally left blank.] 


\section{CASE STUDY}

\subsection{EXISTING SIMULATOR-BASED TRAINING PROGRAMS}

\subsubsection{Introduction}

Two carriers agreed to participate and allow the research team to gather information about their existing truck simulator-based training programs. Participation included access to their truck simulator and training curriculum through consultation and site visits. In addition, access was granted to Class A CDL drivers who recently participated in the carrier's simulator-based training and then took part in a focus group. A non-disclosure agreement was signed by each carrier and the research team's institute. This agreement served to keep carrier identifying information anonymous, while allowing the research team to report information in support of the current effort.

\subsubsection{Carrier Profiles}

The two carriers agreeing to participate in the study were very similar in size and scope of operations. Profiles of both carriers, including fleet sizes and number of drivers, are provided below.

\subsubsection{Carrier $A$}

Carrier A employed or directly contracted over 10,000 Class A CDL drivers. The carrier had a fleet consisting of more than 10,000 power units and 40,000 trailers. In addition, Carrier A maintained more than 30 terminal locations across the continental U.S. Many of these terminal locations offered simulator-based training opportunities to drivers. This carrier reported more than 1,000,000,000 miles logged in their 2007 Motor Carrier Identification Report. Carrier A did not hire drivers who did not possess a valid Class A CDL and did not offer entry-level Class A CDL driver training. However, they did provide new-hire driver finishing programs and regularly scheduled refresher training for their employed and contracted drivers.

\subsubsection{Carrier $B$}

Carrier B employed or directly contracted more than 10,000 Class A CDL drivers. The carrier had a fleet consisting of more than 10,000 power units and 40,000 trailers. Carrier B maintained more than 30 terminal locations across the continental U.S. This carrier offered simulator-based training opportunities to drivers at 35 locations nationwide. This carrier reported more than 1,000,000,000 logged miles in their 2007 Motor Carrier Identification Report. Carrier B offered new and entry-level Class A CDL driver training as well as new-hire and entry-level driver finishing. In addition, the carrier offers regularly scheduled refresher training. New/entry-level Class A CDL truck simulator-based driver training occurred at their fixed-site truck simulator terminal locations. This training program consisted of at least 120 hours of instruction and guided experience lasting 35-45 days per driver. 


\subsubsection{Simulator Information}

\subsubsection{Carrier A Simulator}

Carrier A used an L3 STS I-Sim TranSim VS III driving simulator in their simulator-based training program. These fixed-base simulators used a three-screen display and a partial truckstyle dash based on a reconfigurable glass-panel dash and touch screen controls. Figure 34 displays an image of the glass-panel dash and touch screen controls. Feedback was provided to the driver through a force-feedback steering wheel. A 10-speed shift tower, as well as clutch, brake, and throttle pedals were included. The three image generators provided an approximately 180 degree field-of-view for the driver. Three plasma screens operated at a 1024 x 768 image resolution with a $70 \mathrm{~Hz}$ refresh rate. See Figure 34, Figure 35, and Figure 36 for images of the simulator screen, dash, and truck seat.

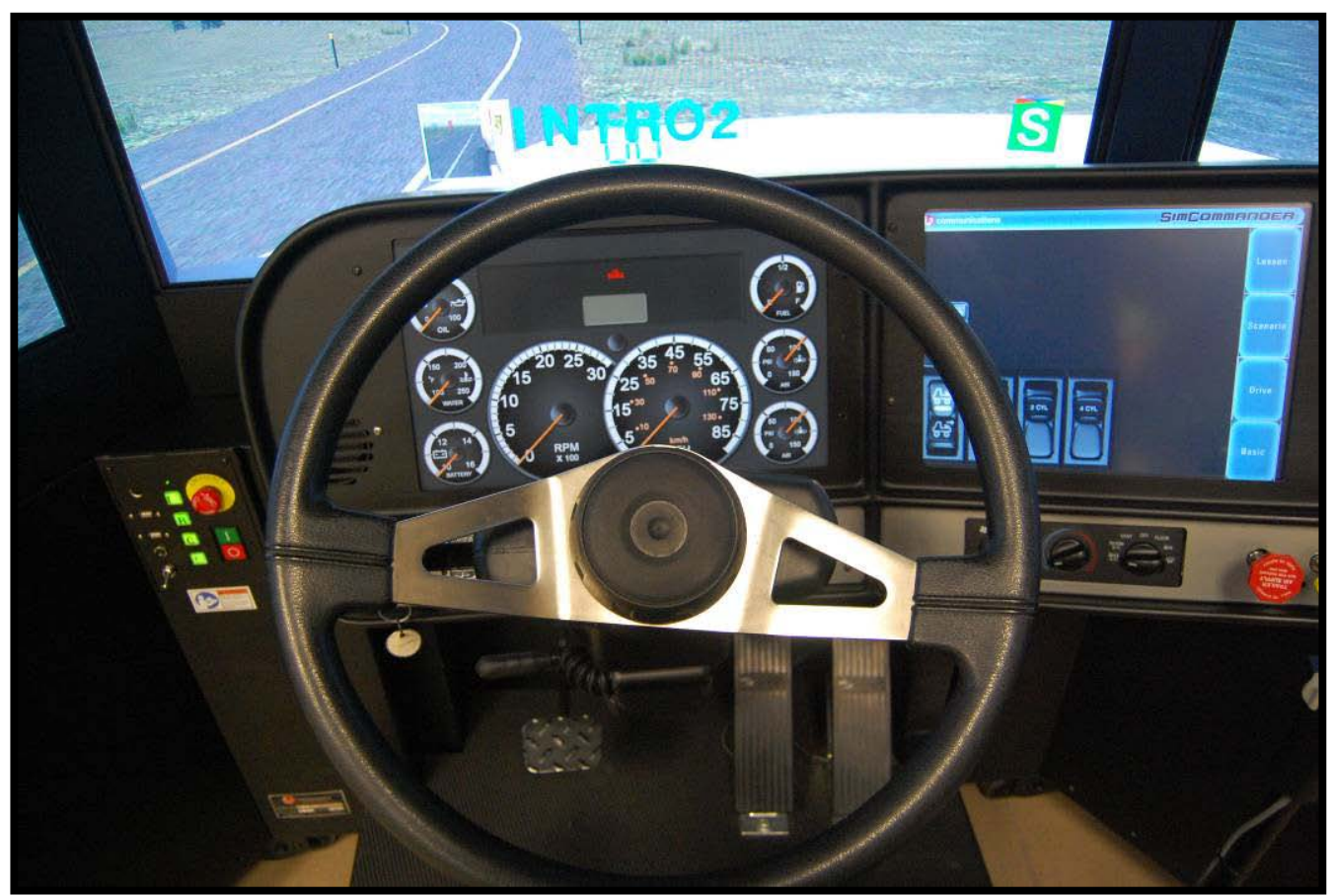

Figure 34. Photo. Glass-panel dash and touch screen control panel used at Carrier A. 


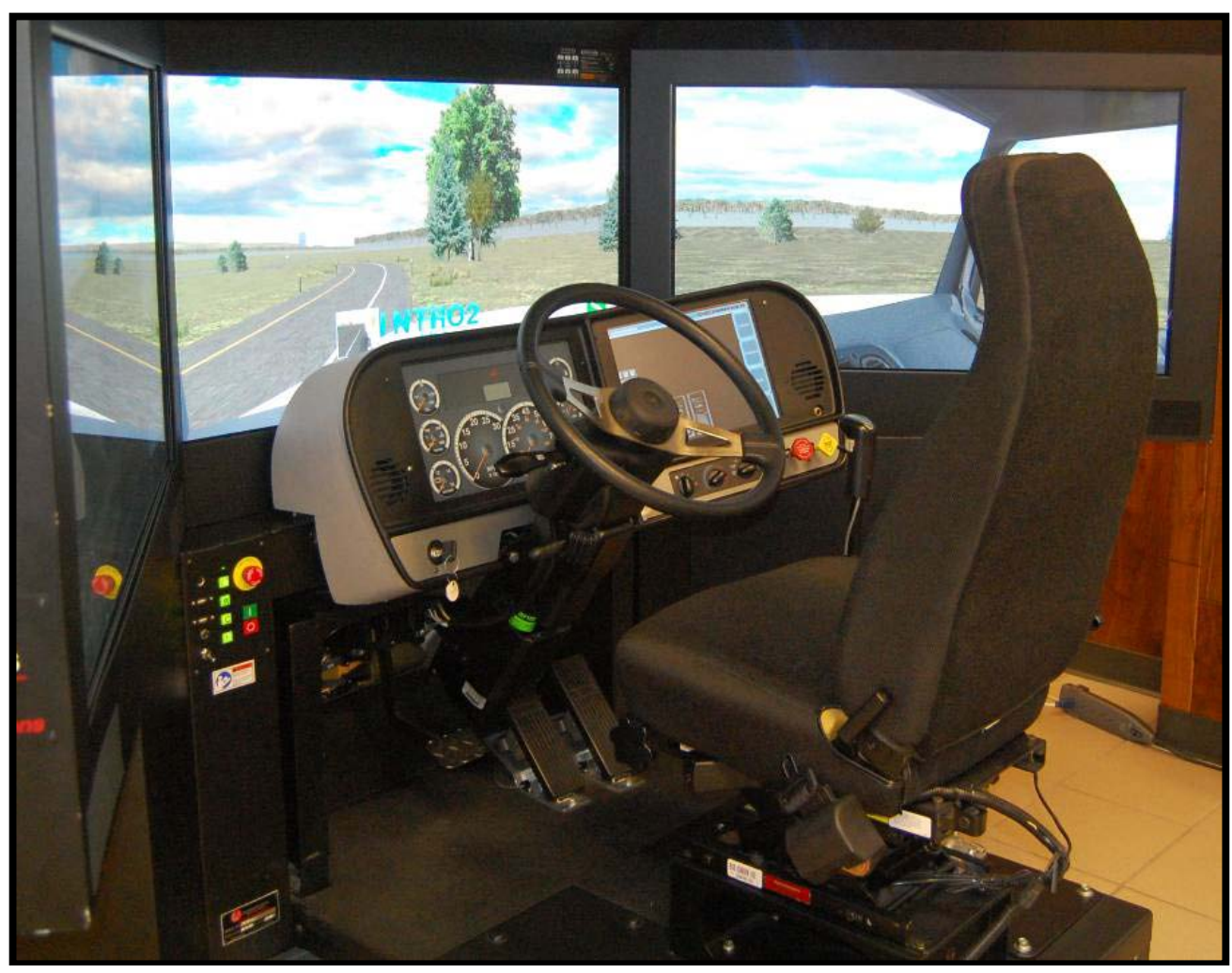

Figure 35. Photo. Simulator screens, dash, and seat used at Carrier A.

The physics of the simulation was configurable between a number of different transmission types, including both automatic and manual transmissions. Figure 36 displays an image of the truck simulator transmission used at Carrier A. The carrier used a standard 10-speed shift configuration in their scenarios. 


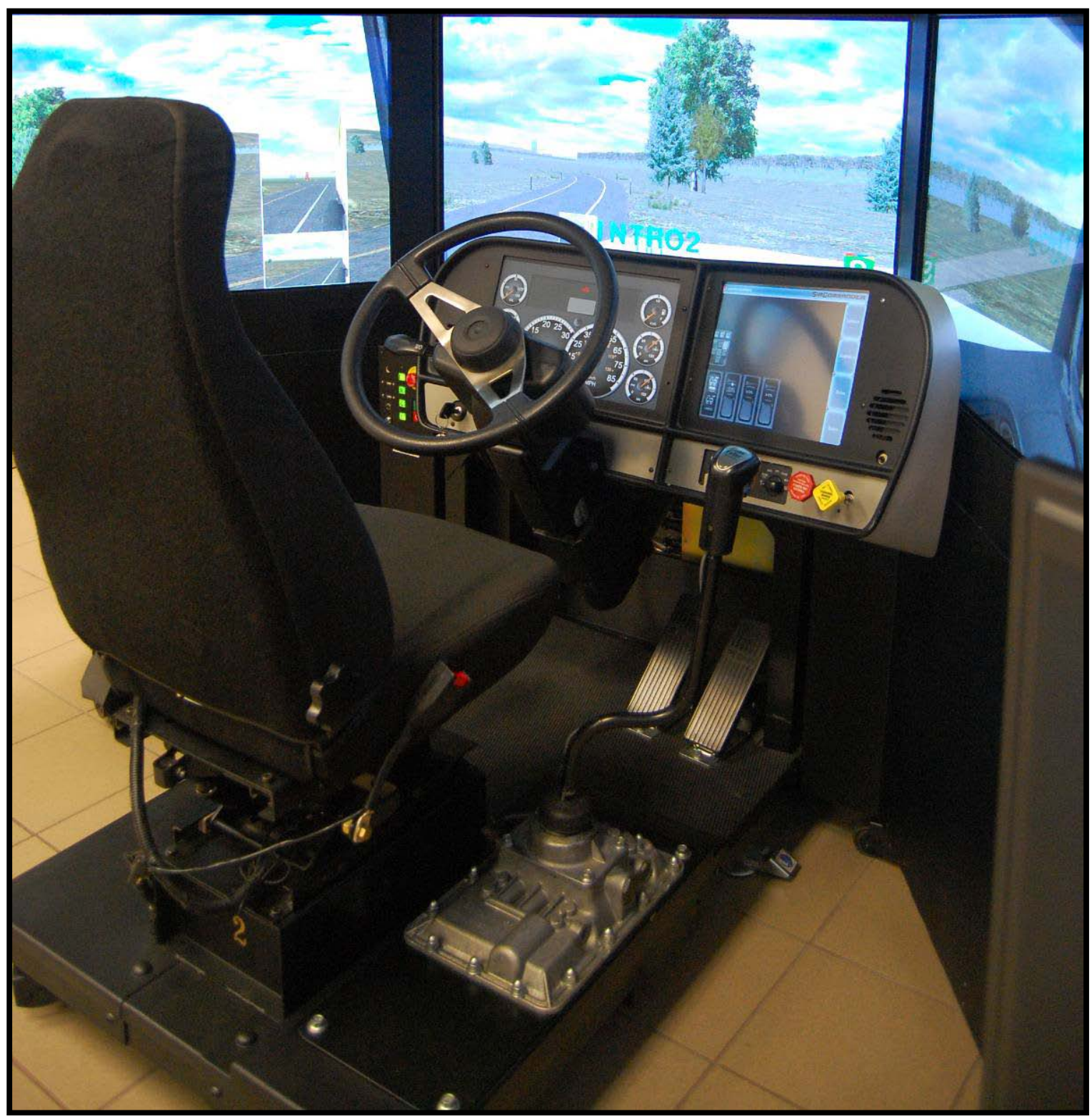

Figure 36. Photo. Simulator shift tower used at Carrier A.

In addition to the fixed-site truck simulators, Carrier A also used mobile truck simulators. These were similar to the fixed-site truck simulators described above; however, the mobile truck simulators were arranged within a standard 48-foot van trailer that could be transported to different locations as needed. The simulator equipment used in the mobile simulator was the same as was used in the fixed-site simulators. The carrier operated three fixed-site and three mobile truck simulators as of the writing of this report.

Carrier A had their truck simulators arranged so the trainer could observe the trainee from the operator's console. At the conclusion of the simulator drive, the trainee and trainer could both view the trainee's performance on an external, large-screen monitor positioned adjacent to the simulator. See Figure 37 for an image of the arrangement of the truck simulator and trainer's monitor. The mobile simulators were arranged in a similar manner. 


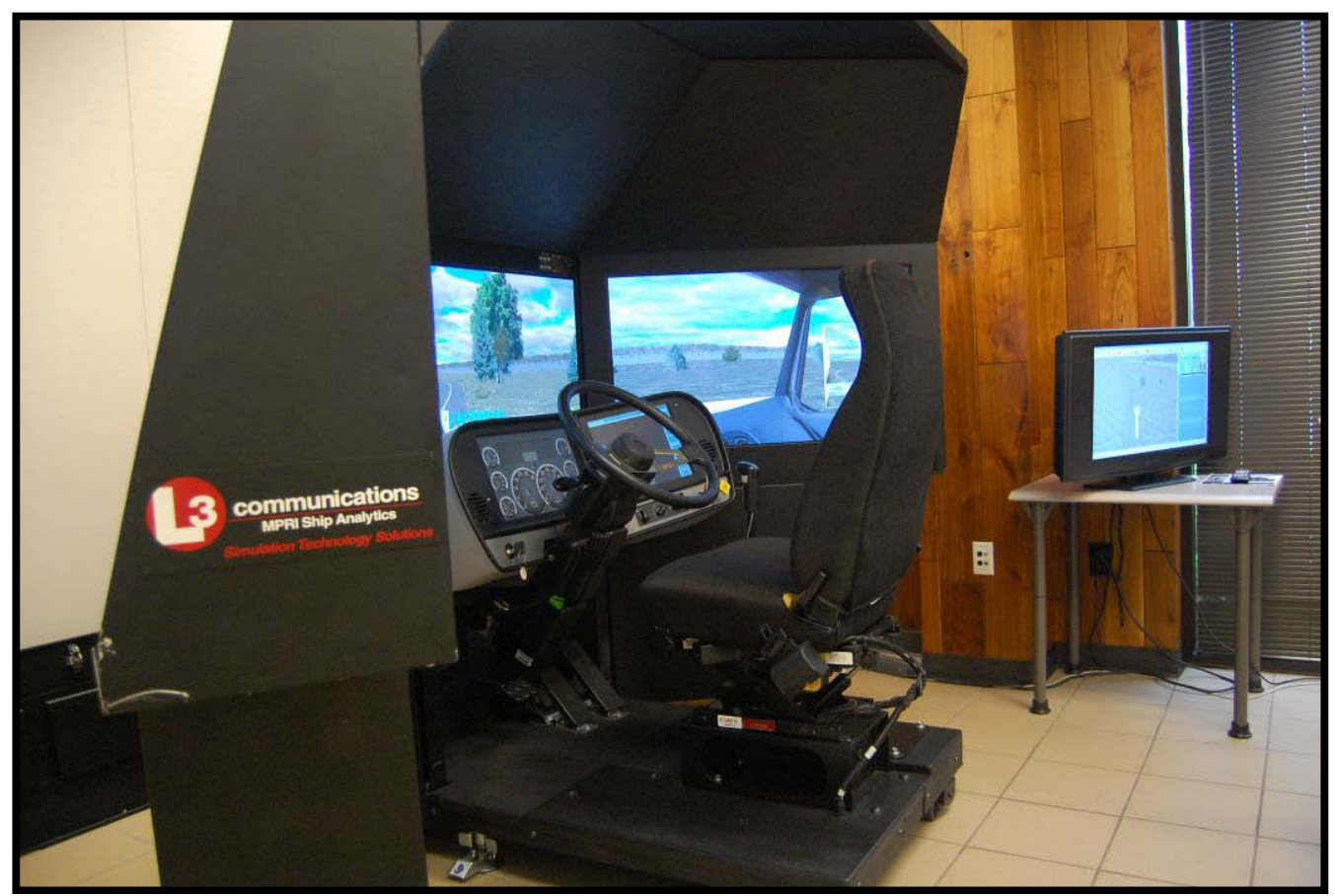

Figure 37. Photo. Simulator and playback monitor used at Carrier A.

\subsubsection{Carrier B Simulator}

Carrier B used a L3 STS I-Sim TranSim VS III driving simulator for their simulator-based training program. These fixed-base simulators used a three-screen display and a partial-truck style dash based on a reconfigurable glass-panel dash and touch screen controls. Feedback was provided to the driver through a force-feedback steering wheel. A 10-speed shift tower, as well as clutch, brake, and throttle pedals were also included. The three image generators provided an approximate 180 degree field-of-view for the driver. Three plasma screens operated at a $1024 \mathrm{x}$ 768 image resolution with a $70 \mathrm{~Hz}$ refresh rate. See Figure 38 for the simulator seat, dash, and screens used at Carrier B. 


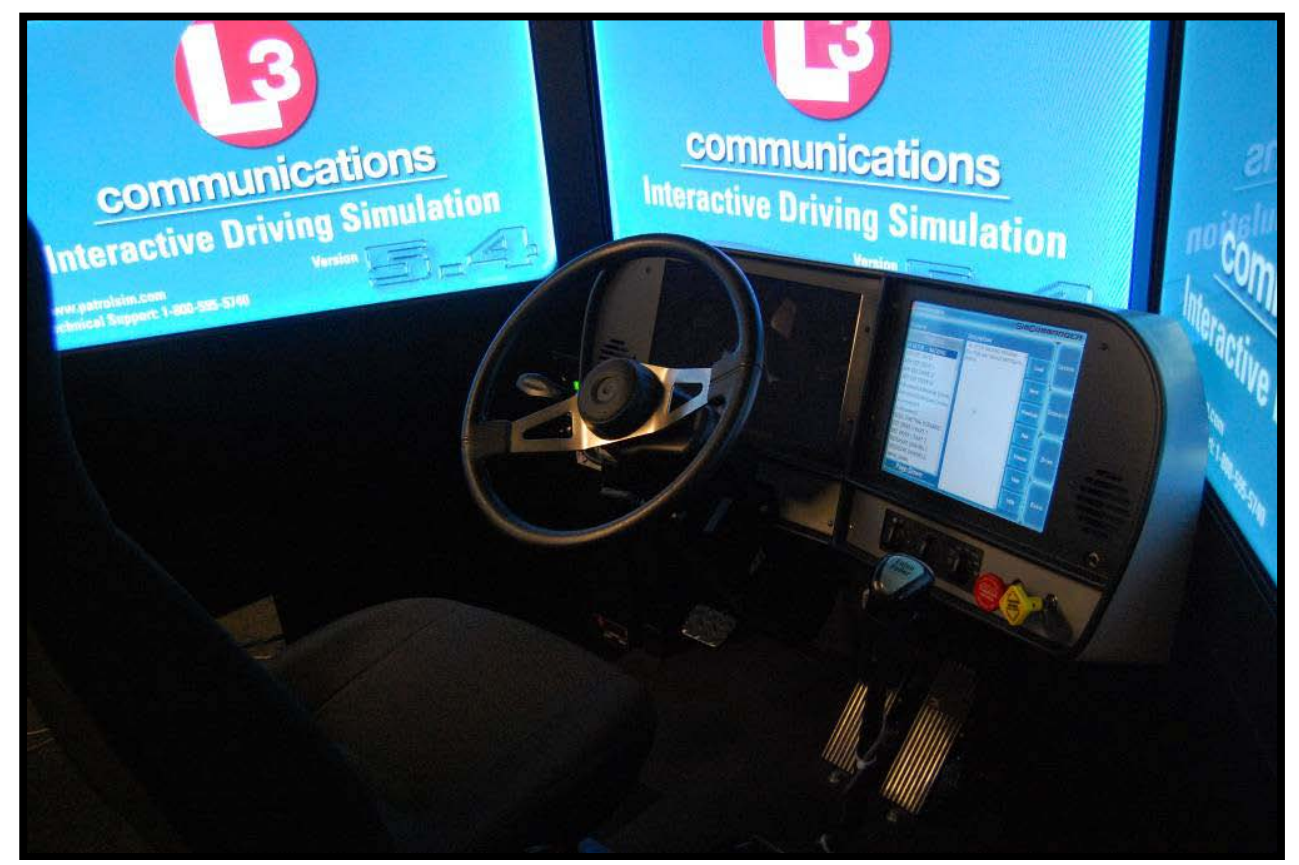

Figure 38. Photo. Simulator seat, dash, and screens used at Carrier B.

The physics of the simulation was configurable between a number of different transmission types (including both automatic and manual transmissions). Although Carrier B primarily used a standard 10-speed shift configuration, an automatic transmission could be used for some initial training exercises that were not specifically related to shifting. Additionally, differing engines, tire sizes, and axle ratios could be specified, although these were not used by Carrier $\mathrm{B}$. The simulation facility at Carrier B was arranged so that four trainees could simultaneously train under the supervision of two trainers. Two simulators were controlled by one operator's console (or OPCON). See Figure 39 for the OPCON arrangement used at Carrier B. 


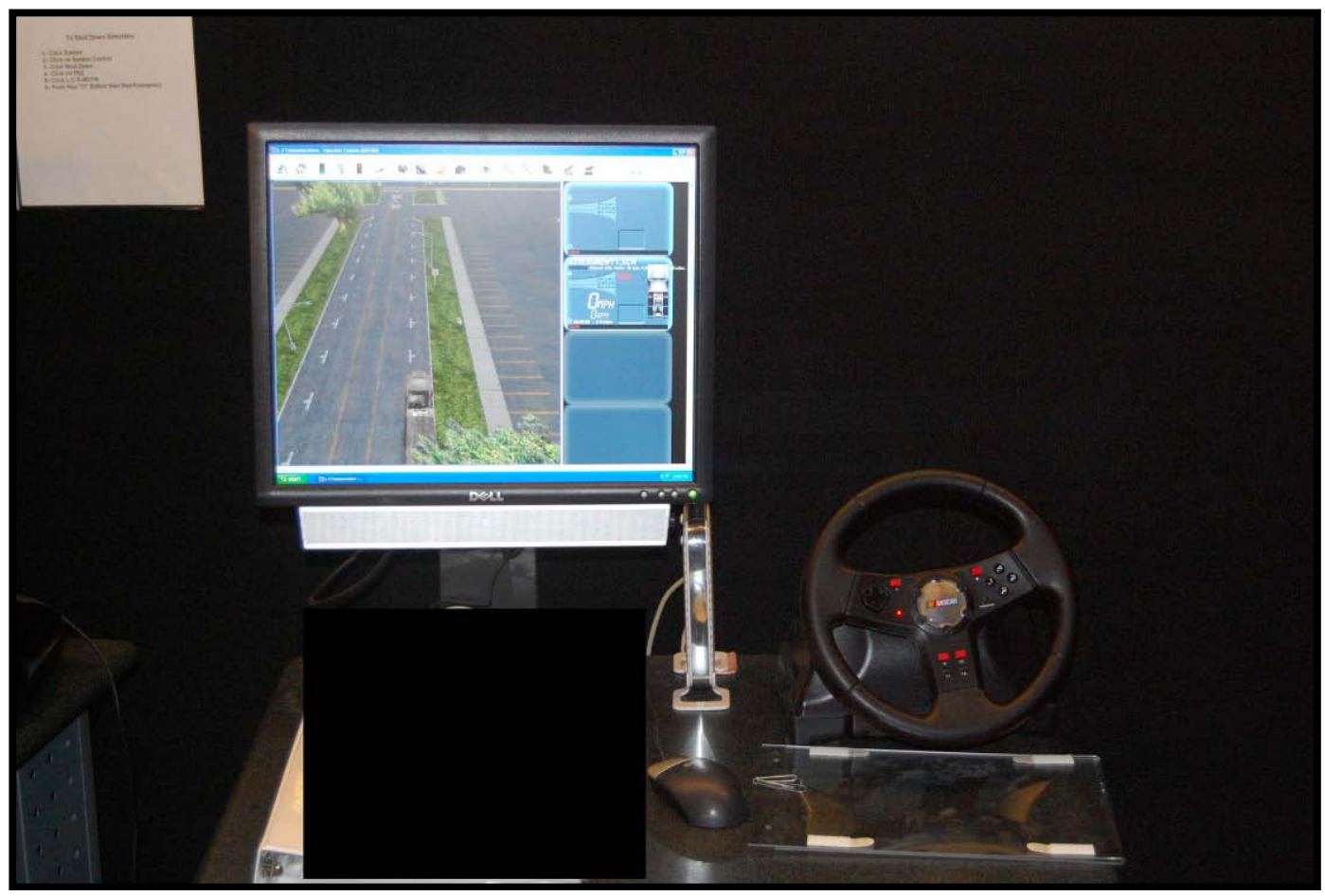

Figure 39. Photo. Simulator OPCON used at Carrier B.

The OPCON was positioned between the two simulators under its control. This arrangement allowed the trainer a clear view of both simulators. The positioning of the OPCON in relation to the truck simulator is shown in Figure 40.

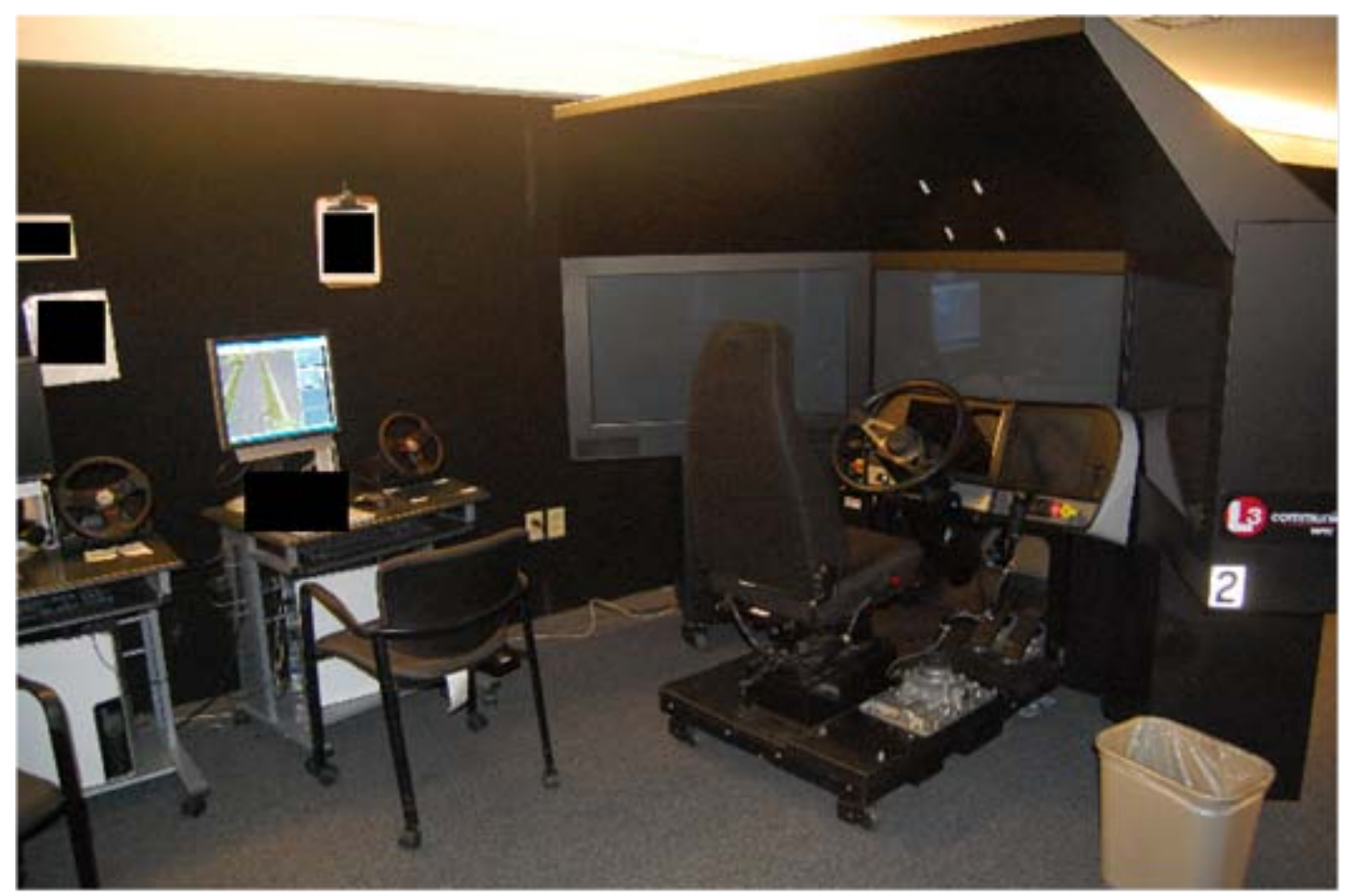

Figure 40. Photo. Operator's console and simulator used at Carrier B. 
Carrier B also operated a mobile truck simulation facility in addition to the fixed-site truck simulator. This mobile simulation facility contained a truck simulator within a trailer that could be transported to customer locations and terminals as needed. Similar to the arrangement used by Carrier A, Carrier B's mobile truck simulator was housed in a standard 48-foot van trailer and used the same simulation equipment as their fixed-site simulators.

\subsubsection{Simulator Training Materials}

\subsubsection{Carrier A Training Materials}

Carrier A's training was oriented towards maintaining and improving the skills of their existing drivers and offering remedial training to drivers following a safety incident which was reported to the company (e.g., crash and/or driving violation). Almost all drivers that were based near a terminal with a simulator training facility were required to undergo quarterly truck simulatorbased training. Drivers with a safety incident(s) were required to complete targeted truck simulator-based training prior to returning to active driving duty. Drivers undergoing training spent approximately 1-2 hours in the truck simulator for regular training and were allowed breaks after 5-20 minutes on the truck simulator. Training was always conducted one-on-one, with a single trainee being observed and coached by a single trainer.

Carrier A used an in-house authored scenario for quarterly driver training. This scenario used a freeway environment. Key concepts of the Smith System (a commercial defensive driving training program) were integrated into the scenario and allowed trainees to practice (and be evaluated on) defensive driving techniques, such as leaving adequate space for emergency lane changes and anticipating the actions of other drivers. The scenario began with the trainee traveling on a four-lane divided highway. The trainee was asked to complete several maneuvers, including lane changes, merges, stopping at the shoulder, and passing other vehicles. During this time, trainees were evaluated for proper vehicle and space control, mirror monitoring, and decision making. Situations with a high likelihood of a crash were included in the scenarios. These included a sudden forced lane change and a pedestrian running into the vehicle's path. In both cases the trainee was forced to make a sudden response to avoid a collision. These situations complemented Carrier A's use of the commercial training program, which placed emphasis on visual scanning and leaving space for emergency maneuvers.

Targeted training typically occurred when a driver had a recorded safety incident or for issues such as improving a driver's fuel mileage. The scenarios used for these training events were customized to the driver's needs, using the simulator's scenario editing program. Approximately 40-50 drivers at Carrier A received quarterly training each week. The exact rate of drivers completing truck simulator training was dependent on driver skill development (trainers were flexible in scheduling drivers for training to accommodate driver needs).

\subsubsection{Carrier B Training Materials}

Carrier B used a blended training approach for entry-level driver training. Blended training combined traditional BTW training with truck simulator-based training. In Carrier B's program, the trainee spent approximately 20 percent of his/her driving time in the simulator, with the remainder of time in BTW driving. The entry-level driver training program, from start to final testing, lasted an average of 35 days per driver. However, due to State law, training at one 
Carrier B training location lasted an additional 10 days. This carrier's truck simulator driving exercises introduced entry-level trainee drivers to situations where basic combination-vehicle driving skills were introduced and mastered by entry-level drivers. These were used as an introduction to many driving techniques and occurred prior to training in real trucks. Most simulated scenarios were conducted while a trainer simultaneously worked with one or two trainees. Trainees typically received an initial briefing prior to the training exercise, and received feedback during the exercise and a post-drive evaluation at the conclusion of the exercise.

Unless specifically requested by a trainee, all exercises at Carrier B were conducted using a standard, double-clutching 10-speed transmission. Each trainee had to reach a criterion of 80 percent efficiency (in terms of correct execution of the skill or maneuver) prior to advancing to the next exercise. Each truck simulator training exercise began with a demonstration of the skill or maneuver by the trainer, followed by the trainee completing the scenario. The scenario could be repeated as needed until the trainer was satisfied with trainee performance. Trainee performance during each simulated scenario was recorded on the simulator's OPCON to be used during trainee evaluations. Trainees received a break every 7-10 minutes.

The simulated scenarios were based on different driving environments. More specifically, basic shifting training was conducted in a skid pad environment, backing exercises were conducted in a simulated warehouse/terminal, and other exercises were conducted in rural, freeway, and/or urban environments. Basic shifting exercises were primarily for entry-level trainees. This exercise covered double-clutching, up-shifting, down-shifting, skip-shifting, and gear recovery (from a missed/poorly executed gear shift). Trainees learned up-shifting through successive approximations. That is, trainees began practicing up-shifting from 2nd to 5th gear at idle and while accelerating, followed by up-shifting from 2nd to 10th gear while at idle and accelerating.

Skip-shifting was introduced after the trainees mastered basic up-shifting. Then, down-shifting and gear recovery were introduced by having trainees begin in 10th gear and power downshift to $5^{\text {th }}$ gear. After the trainee successfully completed basic shifting training, the techniques were practiced in a simulated rural freeway, or urban driving environment. The trainers also allowed the truck simulator to be used as a PTT device during shifting exercises. Trainees with difficulty in locating or shifting gears could use the simulator while the system was powered down to practice the physical movements required for double clutching and shift tower movements (effectively using the simulator as a PTT).

Defensive driving exercises were conducted in a variety of driving environments. These defensive driving exercises began with a trainer-provided demonstration (upon request from the trainee). These simulated scenarios were configured with directional arrows through the simulated environment and provided navigational information to the trainee. The trainee was provided with instructions and feedback before and during the training exercise. Trainers were vigilant in their supervision of trainee performance and provided detailed feedback regarding items such as mirror checks for turns, lane changes, intersections, and straight line backing. These defensive driving exercises also covered signaling, handling unavoidable curb impacts, and increasing awareness of the driving environment (i.e., situational awareness). At the trainer's discretion, the trainee was asked to provide commentary on their defensive driving behaviors throughout the exercise, similar to a think-aloud protocol (Ericksson \& Simon, 1980). 
Carrier B trainers also instructed trainees in the method of snub braking, a technique for braking heavy vehicles at high speeds during descending grades. Snub braking has been demonstrated as the most effective and proper manner of downhill braking (State of Delaware Division of Motor Vehicles, 2005), replacing traditional "light and steady" methods of brake application. Snub braking was taught in two different driving environments. The exercise was conducted with the simulated vehicle configured as a tractor trailer at 80,000 lbs. The first snub braking exercise was during a basic, or empty, driving environment. Trainees began by accelerating to $60 \mathrm{mi} / \mathrm{h}$; once this speed was reached, the grade was adjusted to 6 percent up-grade and the trainee was instructed to select the appropriate gear. Once this basic exercise was completed, the grade was set to 0 percent and information on brake inspection, reading safety signs, and downhill gear selection was covered. Then, the trainee continued with a grade set to a 6 percent down-grade. Snub braking exercises were practiced a minimum of 15 times.

The primary training for off-road slow speed maneuvering occurred on a simulated skid-pad and warehouse/terminal environment. The objective of these scenarios was to develop proper space management using defensive driving techniques, proper backing, and understanding trailer overhang while conducting slow speed maneuvers. The training scenario began with the trainee driving a trailer-free tractor (or bobtail configuration) through a simulated course, using only 1st and 2nd gears. Then, the trainee driver completed a serpentine course with a trailer attached. Backing was covered in the warehouse/terminal environment. The trainee was given an environment with an optimal path overlaid upon the skid pad. See Figure 41 and Figure 42 for an image of the scenario where the optimal path was overlaid on the skip pad. This path informed the trainee on the ideal path to follow to complete an offset backing maneuver. After the trainee successfully completed the offset backing with the ideal path aid, then he or she practiced the maneuver without the preset path.

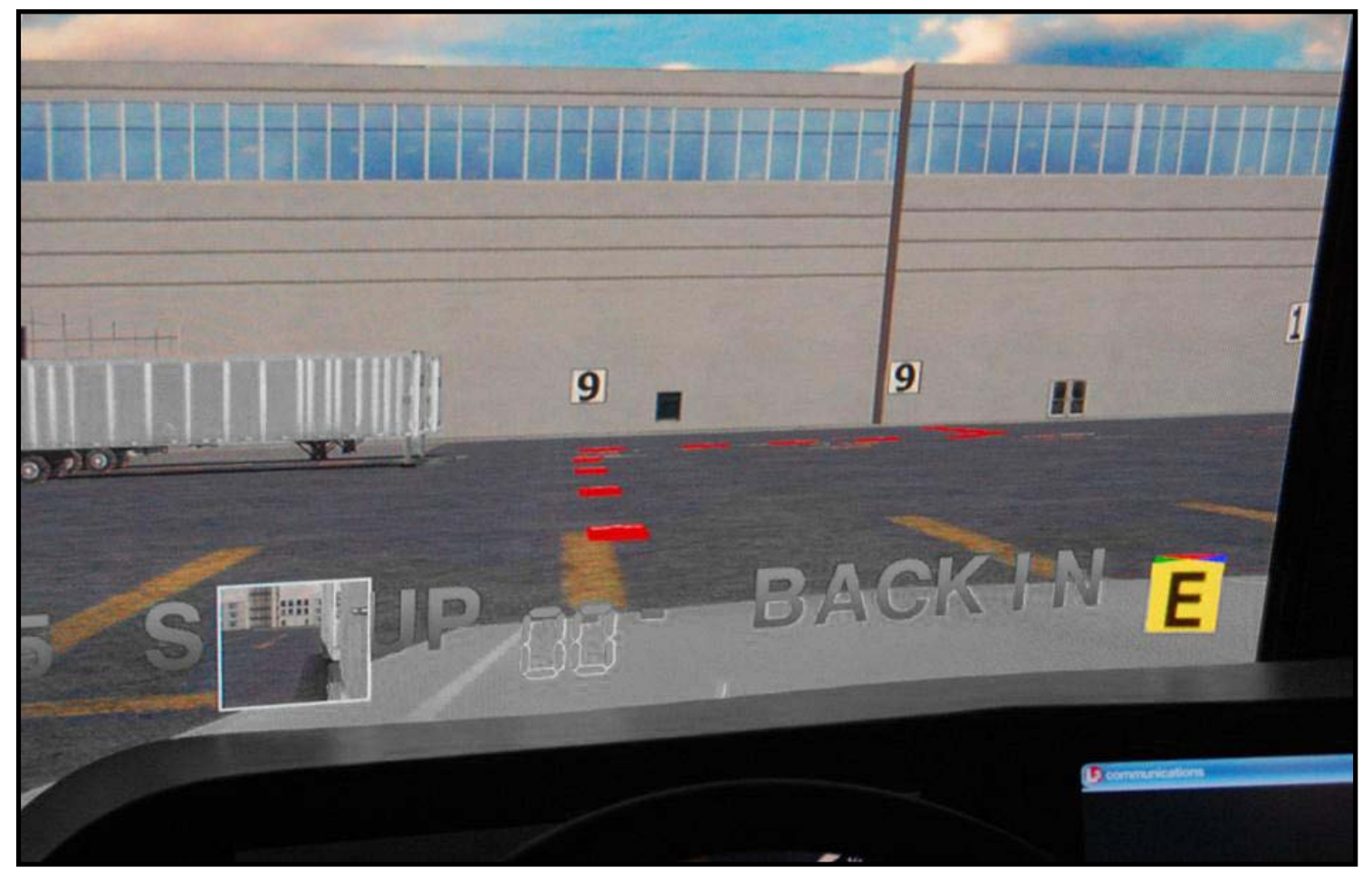

Figure 41. Illustration. Driver's view of introductory backing scenario. 


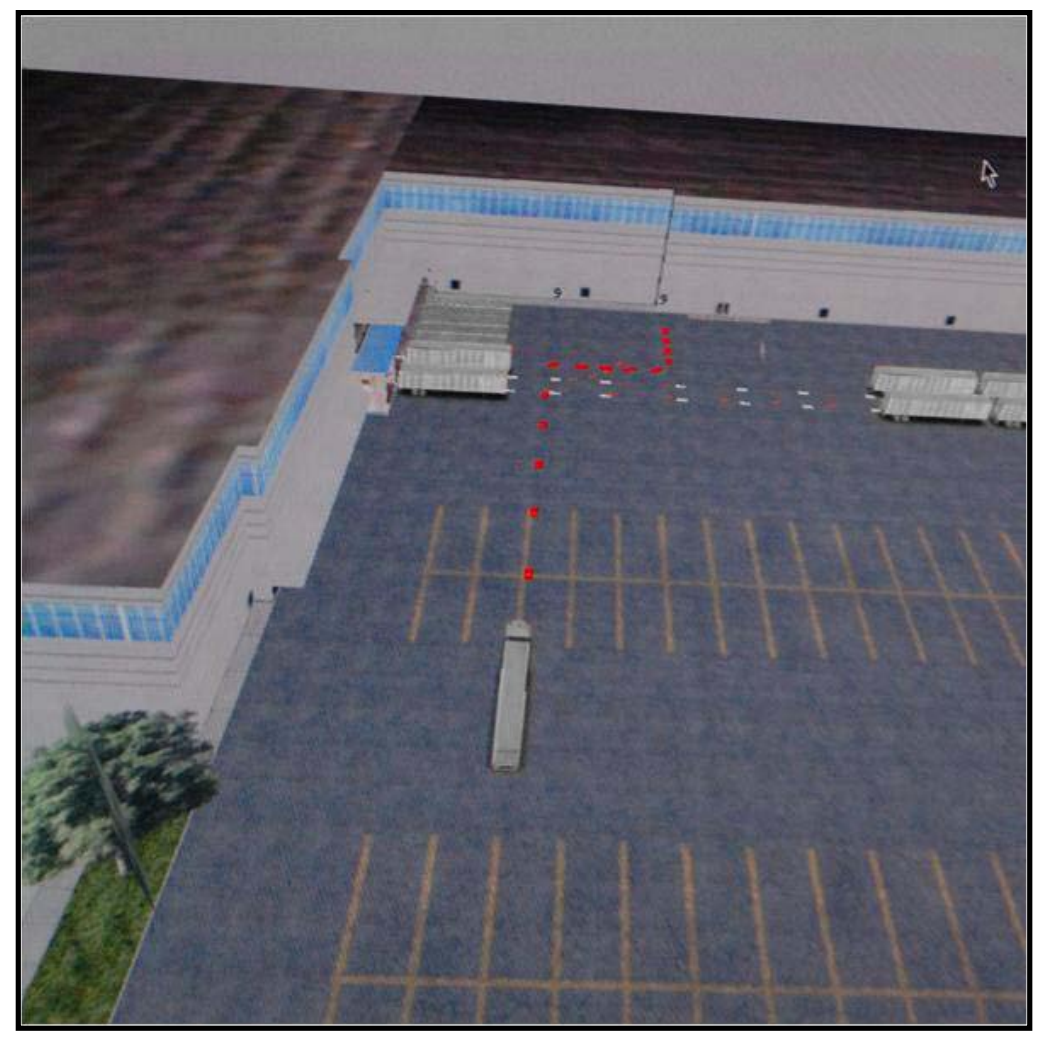

Figure 42. Illustration. Operator's console overhead view of introductory backing scenario.

Skid control was taught using a simulated skid pad or a constant radius curve. For the skid pad maneuvers, trainees were familiarized with the driving environment. Following this, a briefly engaged lock of either the left or right drive axle was simulated (causing the truck to enter into a skid). For constant radius curve maneuvers, trainees first observed a trainer driving the curve at three different speeds: 8-10 mi/h (does not produce a skid), 12-15 mi/h (produces a recoverable skid), and $20 \mathrm{mi} / \mathrm{h}$ (produces an unrecoverable skid). Then, the trainee practiced driving the constant radius curve at each of the three speeds. The recoverable skid was repeated, as needed, to allow trainees to become familiar with skid recovery in these conditions. Weather conditions were also varied during skid control training. Icy conditions were used with a drive axle lock to simulate a skid (with proper recovery as the training objective).

\subsubsection{Truck Simulator Effectiveness}

\subsubsection{Carrier A}

Data Set and General Remarks: Based on agreements in place with Carrier A, information regarding drivers' history was obtained for further analysis. This information consisted of seven data files with information on driver training, mileage logged, carrier recorded crashes (and their associated costs), and driver tenure. These data were protected under the non-disclosure agreement between the carrier and the research team. Note that data on the effectiveness of simulator truck training was only obtained from Carrier A. Additionally, these drivers should not be considered as entry-level, or novice, drivers; Carrier A does not hire drivers who do not already possess a valid Class A CDL. Thus, all drivers included in this data set should be considered to hold some level of commercial driving experience. 
Using the driver ID number associated with each case or instance in each data file, drivers were matched across files and all data were combined into a single database for all analyses. Two issues, training type and data collection periods, must be noted regarding the data obtained from Carrier A. First, Carrier A exposed drivers to one of three different training types, including: simulator (training primarily occurred via a truck simulator), BTW (training primarily occurred in a real tractor-trailer), and blended training (a combination of simulator and BTW). Drivers at one location received blended training, while the other locations included simulator or BTW training. Therefore, only drivers with both similar locations and training types were included in the data set. Additionally, interactions between training type and driver type were not able to be fully explored within the extant data. Second, the data set did not indicate an end date for data collection. However, based on conversations with a representative at Carrier A, this date was set to October 1, 2007. Thus, data were collected for miles logged by each driver between his or her date of hire and termination or end of data collection on October 1, 2007 (whichever occurred first).

The complete data set included 2,459 drivers. The majority of drivers were listed as having received blended training, with fewer drivers receiving either BTW or simulator training. The majority of drivers were over-the-road or regional drivers, reflecting Carrier A's typical routes. Table 58 displays the distribution of training and driver types across all 2,459 drivers in the data set.

Table 58. Number of Drivers in the Analysis by Training Type and Driving Route

\begin{tabular}{|l|r|r|r|r|}
\hline $\begin{array}{c}\text { Training } \\
\text { Type }\end{array}$ & \multicolumn{1}{c|}{$\begin{array}{c}\text { Local } \\
\text { Drivers }\end{array}$} & $\begin{array}{c}\text { Over-the-Road } \\
\text { Drivers }\end{array}$ & $\begin{array}{c}\text { Regional } \\
\text { Drivers }\end{array}$ & \multicolumn{1}{c|}{ Total } \\
\hline Blended & 114 & 979 & 314 & 1,407 \\
\hline BTW & 7 & 591 & 336 & 934 \\
\hline Simulator & 1 & 98 & 19 & 118 \\
\hline Total & 122 & 1,668 & 669 & 2,459 \\
\hline
\end{tabular}

In order to determine the effects of training on tenure and efficiency, the data were analyzed using a survival/failure analysis. Survival/failure analysis is used to determine a population's characteristics over time, especially when some members of the population may not remain active throughout the data collection period (Woodward, 2005). This was chosen as the most appropriate analytical strategy as the method produces a survival time, but also may be used to illustrate differences between groups. Additionally, not all individual drivers remained in the carrier's employment throughout the entire study period, making the use of other statistical techniques (such as ANOVA) problematic due to missing cases. Even with this consideration, the results of the survival/failure analysis should only be viewed as a description of events occurring within the measurement period (e.g., a training group without a crash during the measurement period may still become involved in a crash, however a crash was not observed within the measurement period) in the data provided by the carrier. These group differences are described in terms of hazard ratios (odds ratios), which allow for relative comparisons between groups in the analysis. The nature of each analysis, and its associated data, is described within each section below. 
Driver Tenure: Driver tenure may be seen as a reflection of the efficiency of a carrier's operations. Increasing the tenure of a driver reduces the costs required to recruit and hire replacement drivers, yielding increases in operational efficiency. Thus, a possible relationship between driver training and tenure is of great importance to a carrier. In this analysis, driver tenure was examined in terms of the temporal duration of driver tenure with and the number of miles logged by the driver during tenure.

\section{Duration of Driver Tenure}

A survival/failure analysis was conducted on the driver tenure data. Overall, 45.43 percent of drivers remained in the carrier's employment through the end of data collection (October 1 , 2007). Of the original 2,459 drivers in the study, 1,344 drivers were no longer employed by Carrier $\mathrm{A}$ at the end of data collection. Table 59 provides the descriptive statistics for driver tenure across each type of training. As shown in Table 59, drivers who received simulator training (140 days) had the longest tenure, followed by blended and BTW training (at 119 and 98 days), respectively. Note that the reason for driver termination was not known. The driver may have resigned, retired, or been terminated by the carrier for some unspecified reason.

Table 59. Descriptive Statistics for Driver Tenure

\begin{tabular}{|l|r|r|r|r|r|r|r|}
\hline Training Type & \multicolumn{1}{c|}{$\boldsymbol{N}$} & \multicolumn{1}{c|}{ Mean } & \multicolumn{1}{c|}{ SD } & \multicolumn{1}{c|}{ Min } & \multicolumn{1}{c|}{ Max } & \multicolumn{1}{c|}{$\mathbf{L C L}^{\mathbf{1}}$} & $\mathbf{U C L}^{2}$ \\
\hline Blended & 1,407 & 118.60 & 78.81 & 1 & 271 & 114.48 & 122.72 \\
\hline BTW & 934 & 98.06 & 72.66 & 2 & 271 & 93.39 & 102.72 \\
\hline Simulator & 118 & 140.13 & 82.75 & 8 & 269 & 125.04 & 155.21 \\
\hline
\end{tabular}

Note: Driver tenure expressed in days. 1. Lower 95 percent Confidence Limit, 2. Upper 95 percent Confidence Limit

Hazard ratios were calculated as part of the survival/failure analysis. This served as a description of the effect of training type on driver tenure and may be considered an estimate of relative risk of termination. Training type was considered the covariate in the analysis. The comparison, or control, training type was BTW training as this was the standard type of training employed in CMV operations. Thus, BTW training was compared to both the blended and simulator training to assess if training type had an effect on driver tenure.

All calculations of hazard ratios for training type used BTW training as the reference group; therefore, the hazard for the other training groups was compared to the BTW group to assess the ratios. Hazard ratios greater than 1.0 indicate that training type negatively affected tenure (larger hazard ratios indicate a shorter tenure with the company), while hazard ratios less than this value indicate that training type positively affected tenure (smaller hazard ratios indicating a longer tenure with the company). A hazard ratio of exactly 1.0 indicated no effect of training type on tenure. Both the simulator and blended training differed significantly as compared to BTW training. Drivers in the simulator training had the lowest hazard ratio (hazard ratio $=.66, \chi^{2}{ }_{(1)}=$ $9.75, p=.0018)$. Thus, these drivers were 34 percent less likely to leave the carrier's employment (e.g., terminated, resigned) during data collection than were BTW drivers. Blended training also resulted in a lower relative risk of termination as compared to BTW training (hazard ratio $\left.=.82, \chi_{(1)}^{2}=12.78, p=.0003\right)$. Thus, these drivers were 18 percent less likely to leave the carrier's employment during data collection than were BTW drivers. 
A Wald chi-squared test was used to assess for differences between simulator and blended training. The chi-square was not significant $\left(\chi_{(1)}^{2}=2.66, p=0.10\right)$. There was no significant difference between simulator and blended training. The results of the survival/failure analysis, including the hazard ratios, chi-square tests, and confidence intervals, are shown in table 60.

Table 60. Survival/Failure Analysis for Driver Tenure

\begin{tabular}{|l|r|r|r|r|r|r|r|r|}
\hline $\begin{array}{c}\text { Training } \\
\text { Type }\end{array}$ & $d f$ & $\begin{array}{c}\text { Parameter } \\
\text { Estimate }\end{array}$ & SE & $\begin{array}{c}\text { Chi- } \\
\text { Square }\end{array}$ & $\begin{array}{c}p- \\
\text { Value }\end{array}$ & $\begin{array}{c}\text { Hazard } \\
\text { Ratio }\end{array}$ & $\begin{array}{c}\text { Hazard } \\
\text { Ratio } \\
\text { LCL }\end{array}$ & $\begin{array}{c}\text { Hazard } \\
\text { Ratio } \\
\text { UCL }\end{array}$ \\
\hline Simulator & 1 & -0.42 & 0.13 & 9.75 & 0.0018 & 0.66 & 0.51 & 0.86 \\
\hline Blended & 1 & -0.20 & 0.06 & 12.78 & 0.0003 & 0.82 & 0.73 & 0.91 \\
\hline
\end{tabular}

\section{Miles Logged During Tenure}

Driver tenure was also measured by miles driven (as recorded in the driver's logbook). As above, miles driven until the study end date were included in the survival/failure analysis. This analysis included 2,145 drivers (sufficient data were not available for the remaining 314 drivers). Table 61 provides the descriptive statistics for driver mileage across each type of training (for all 2,145 drivers). Drivers who received simulator training $(M=36,016$ miles, $S D=23,307)$ had the longest tenure as measured in logged miles driven, followed by blended $(M=26,819$ miles, $S D=$ $21,045)$ and BTW training $(M=21,275$ miles, $S D=18,527)$, respectively. Of these drivers, 44.34 percent continued to record miles driven in their logbooks until the end of data collection.

Table 61. Descriptive Statistics for Miles Logged During Tenure

\begin{tabular}{|l|c|c|c|c|c|c|c|}
\hline \multicolumn{1}{|c|}{ Training } & $\boldsymbol{n}$ & Mean & SD & Min & Max & LCL & UCL \\
\hline Blended & 1,229 & 26,819 & 21,045 & 11 & 95,853 & 25,641 & 27,997 \\
\hline BTW & 810 & 21,275 & 18,527 & 3 & 96,515 & 19,998 & 22,553 \\
\hline Simulator & 106 & 36,016 & 23,307 & 21 & 84,933 & 31,527 & 40,504 \\
\hline
\end{tabular}

Note: Driver tenure expressed in days.

Hazard ratios were calculated as part of the survival/failure analysis of miles driven during tenure. This serves as a description of the effect of training type on miles logged and may be considered an estimate of relative risk in terms of miles logged. As above, training type was considered the covariate in the analysis. The comparison, or control, training type was BTW training, as this was the standard type of training employed in CMV operations. Thus, BTW training was compared to both blended and simulator training to assess if training type had an effect on driver tenure.

Table 62 displays the hazard ratios by training type for miles logged. As the comparison, or control variable, BTW training had a hazard ratio of one (1.0). The hazard analysis results for miles logged during driver tenure were similar in outcome to the results of the hazard analysis on tenure measured in days (shown in Table 60). Both simulator and blended training differed significantly as compared to BTW training. Drivers in the simulator training group had the lowest hazard ratio (hazard ratio $=.56, \chi_{(1)}^{2}=16.13, p<.0001$ ). These drivers were 44 percent more likely to log a greater number of miles during the study period as compared to BTW drivers. Blended training also resulted in logging more miles driven during the study period as 
compared to BTW training (hazard ratio $=.80, \chi_{(1)}^{2}=13.83, p=.0002$ ). Drivers who underwent the blended training were 20 percent more likely to log more miles during data collection than were BTW drivers.

A Wald chi-squared test was used to assess for differences between simulator and blended training. A significant result $\left(\chi^{2}(1)=6.27, p=0.01\right.$ ) was present, indicating that drivers in simulator training were significantly more likely to log a greater number of miles during the study period than drivers in blended training. The results of the survival/failure analysis, including the hazard ratios, chi-squares, and confidence intervals, are summarized in Table 62.

Table 62. Survival/Failure Analysis for Miles Logged During Tenure

\begin{tabular}{|l|c|c|c|c|c|c|c|c|}
\hline $\begin{array}{c}\text { Training } \\
\text { Type }\end{array}$ & $d f$ & $\begin{array}{c}\text { Parameter } \\
\text { Estimate }\end{array}$ & SE & $\begin{array}{c}\text { Chi- } \\
\text { Square }\end{array}$ & $\boldsymbol{p}$-Value & $\begin{array}{c}\text { Hazard } \\
\text { Ratio }\end{array}$ & $\begin{array}{c}\text { Hazard } \\
\text { Ratio } \\
\text { LCL }\end{array}$ & $\begin{array}{c}\text { Hazard } \\
\text { Ratio } \\
\text { UCL }\end{array}$ \\
\hline Simulator & 1 & -0.58 & 0.15 & 16.13 & $<0.0001$ & 0.56 & 0.42 & 0.74 \\
\hline Blended & 1 & -0.23 & 0.06 & 13.83 & 0.0002 & 0.80 & 0.71 & 0.90 \\
\hline
\end{tabular}

Crash Involvement: A crash was defined as any event listed as an "accident" in the data set provided by Carrier A. No further information on the carrier's definition of accident was available. The majority of drivers had no crash events. Approximately 93, 96, and 87 percent of drivers in the blended, BTW, and simulator training types, respectively, had zero crashes. The frequency of crashes per driver across each training type is depicted in Figure 43. Note that the operational definition of "accident" was not known. These "accidents" could include non-fault and at-fault events on the part of the truck driver. 


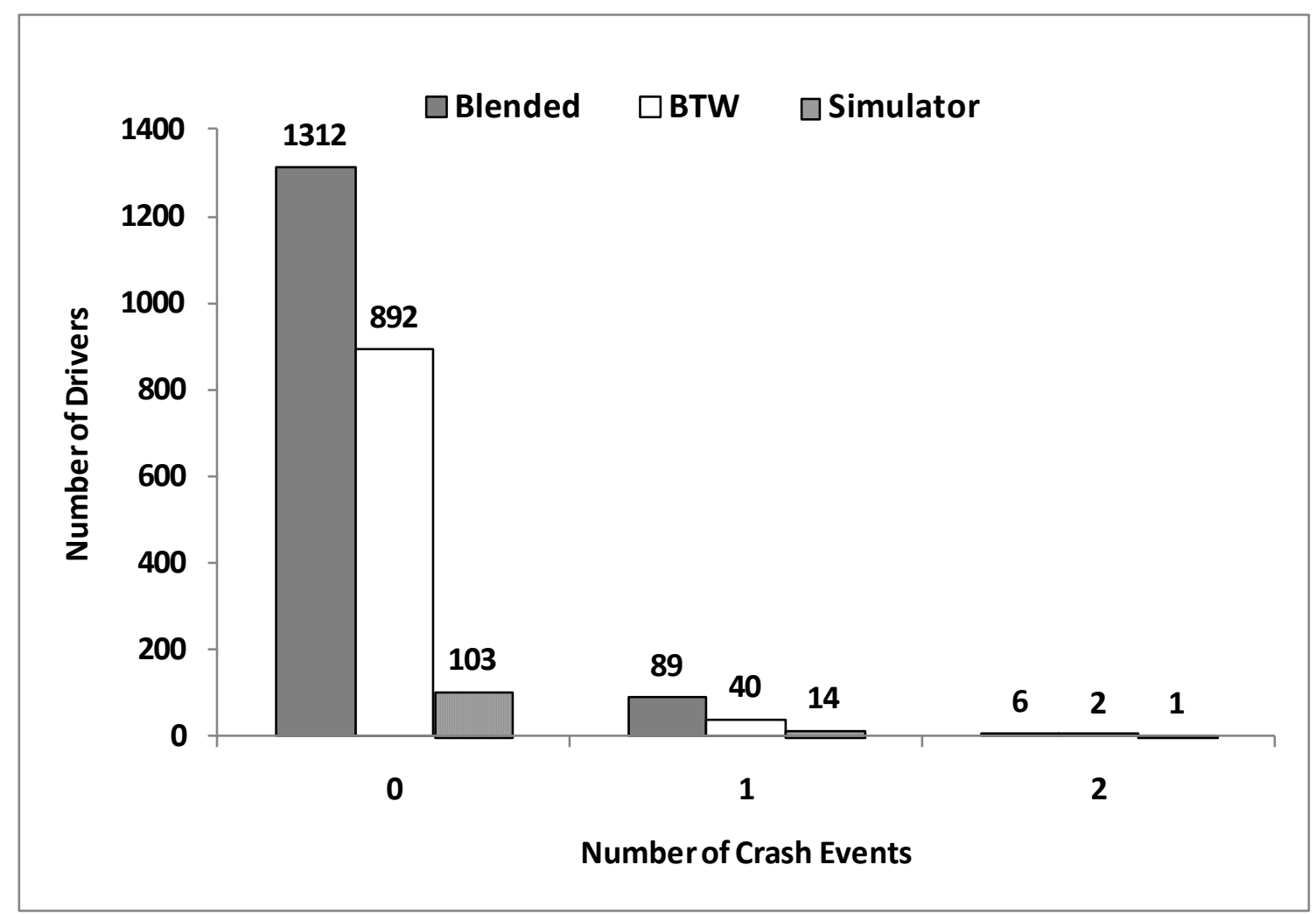

Figure 43. Graph. Frequency of crash events per driver by training type.

Using the mileage recorded over driver tenure and the crashes recorded for each driver, crash rates per 1,000 miles driven were calculated. In order to limit the influence of drivers with a low number of total miles recorded during their tenure, any driver with fewer than 10,000 miles (a value chosen as a representative minimum for drivers in the company) was removed from the analysis. The remaining 1,469 drivers were analyzed by training type.

Blended drivers had the highest crash rate per thousand miles $(M=0.0059, S D=0.0187$, $n=878)$, followed by simulator-trained drivers $(M=0.0056, S D=0.0136, n=89)$ and BTWtrained drivers $(M=0.0038, S D=0.0153, n=502)$. Descriptive statistics for each training group's crash rate per thousand miles is presented in table 63. An ANOVA was performed on the effect of training type on the crash rate per 1,000 miles driven. No significant differences were present between groups $\left(F_{(2,1466)}=2.40, p=0.09\right)$. The non-significance of this finding may be due to the relatively imbalanced number of drivers in each condition ( $n$ of 878, 502, and 89, for the blended, BTW, and simulator training, respectively). However, in this analysis, Levene's test did not indicate a violation of the homogeneity of variance assumption $(p=0.38)$. 
Table 63. Crash Rate per Thousand Miles by Training Type

\begin{tabular}{|l|c|c|c|c|c|c|c|}
\hline Training Type & $\boldsymbol{N}$ & Mean & SD & Min & Max & LCL & UCL \\
\hline Blended & 878 & 0.0059 & 0.0187 & 0.0000 & 0.1620 & 0.0047 & 0.0072 \\
\hline BTW & 502 & 0.0038 & 0.0153 & 0.0000 & 0.1831 & 0.0025 & 0.0052 \\
\hline Simulator & 89 & 0.0056 & 0.0136 & 0.0000 & 0.0671 & 0.0028 & 0.0085 \\
\hline
\end{tabular}

\section{Time to Crash}

Time (in days) to the first carrier-recorded crash was assessed for each training type by survival/failure analysis. This analysis included data from all 2,459 drivers. Of these drivers, 93.82 percent (2,307 drivers) had no crash event recorded through the end of data collection. Overall, 6.18 percent of drivers (152 drivers) had a crash during the data collection period. Descriptive statistics for time to crash (in days) after participating in training are provided in Table 64. Drivers who received blended training had an average time to crash of 48 days ( $S D=31$ days), while drivers that received BTW and simulator training had an average time to crash of $46(S D=30$ days $)$ and 48 days $(S D=34$ days $)$, respectively.

Table 64. Descriptive Statistics for Time to Crash

\begin{tabular}{|l|c|c|c|c|c|c|c|}
\hline Training Type & $\boldsymbol{n}$ & Mean & SD & Min & Max & LCL & UCL \\
\hline Blended & 95 & 48.11 & 31.37 & 4 & 140 & 41.72 & 54.49 \\
\hline BTW & 42 & 46.05 & 30.28 & 5 & 120 & 36.61 & 55.48 \\
\hline Simulator & 15 & 47.67 & 34.38 & 5 & 122 & 28.63 & 66.71 \\
\hline
\end{tabular}

Hazard ratios were calculated as part of the survival/failure analysis of days employed until a crash was recorded. This served as an assessment of the effect of training type on crash involvement and may be considered an estimate of relative risk. As with the previously described analysis, training type was considered the covariate in the analysis. The comparison, or control, training type was BTW training, as this was the standard type of training employed in CMV operations. BTW training was compared to both the blended and simulator training to assess if training type had an effect on time to first crash.

Drivers in the simulator training group had a significantly greater hazard ratio (hazard ratio = 2.63, $\left.\chi_{(1)}^{2}=10.11, p=.0015\right)$ compared to BTW-trained drivers. These drivers were 2.63 times more likely to be involved in a crash earlier in their tenure than were non-simulator drivers. Drivers in the blended training group did not significantly differ from drivers in the BTW training group (hazard ratio $=1.27, \chi_{(1)}^{2}=1.57, p=.2101$. A Wald chi-squared test was used to assess for differences between simulator and blended training. A significant result, $\chi_{(1)}^{2}=6.60$, $p=0.01$ was present, indicating that simulator drivers were significantly more likely than blended drivers to have a crash earlier in their tenure. Significant differences were present between simulator-trained drivers and both blended and BTW drivers, with simulator-trained drivers more likely to be involved in a crash event earlier in their tenure as compared to the other two groups. The results of the survival/failure analysis are summarized in Table 65. 
Table 65. Survival/Failure Analysis for Time to Crash by Training Type

\begin{tabular}{|l|c|c|c|c|c|c|c|c|}
\hline $\begin{array}{c}\text { Training } \\
\text { Type }\end{array}$ & $\boldsymbol{d f}$ & $\begin{array}{c}\text { Parameter } \\
\text { Estimate }\end{array}$ & SE & $\begin{array}{c}\text { Chi- } \\
\text { Square }\end{array}$ & $\begin{array}{c}\boldsymbol{p} \text { - } \\
\text { Value }\end{array}$ & $\begin{array}{c}\text { Hazard } \\
\text { Ratio }\end{array}$ & $\begin{array}{c}\text { Hazard } \\
\text { Ratio } \\
\text { LCL }\end{array}$ & $\begin{array}{c}\text { Hazard } \\
\text { Ratio } \\
\text { UCL }\end{array}$ \\
\hline Simulator & 1 & -0.97 & 0.30 & 10.11 & 0.0015 & 2.63 & 1.45 & 4.77 \\
\hline Blended & 1 & -0.24 & 0.19 & 1.57 & 0.2101 & 1.27 & 0.87 & 1.85 \\
\hline
\end{tabular}

\section{Cost Per Crash}

The cost per crash was examined for differences across training types. Due to the large variation in the number of cases across training types and the number of censored cases (drivers who terminated employment prior to the end of data collection) in the data, an ANOVA did not provide any more insight than the comparisons of means and confidence intervals. Drivers with simulator training had the lowest average cost per crash, with a cost per crash of approximately $\$ 460$, while drivers in the BTW and blended training groups had an average cost per crash of approximately $\$ 2,700$ and $\$ 4,000$, respectively (Table 66 ). However, two caveats are necessary when interpreting these results. Although any driver with a crash was included in the analysis (152 drivers), there were a number of crashes with a cost of zero $(\$ 0.00)$ recorded in the data set, which resulted in the minimum observed value of $\$ 0.00$. Additional detail was not available for these zero-cost crashes; however, as carrier-recorded events they were left in the analysis as many carriers' policies allow drivers a specific number of crash events before the driver's employment is terminated. Additionally, one outlier (a statistically high value) was present in the blended training. This value was a single crash with a cost of over $\$ 200,000$, which artificially influenced the average cost per crash for the blended group. Note that the cost data provided by Carrier A was an estimate and was not operationally defined. These numbers could include direct or indirect costs, or costs associated with both.

Table 66. Descriptive Statistics for Cost of Crash

\begin{tabular}{|l|c|c|c|c|c|c|c|}
\hline $\begin{array}{c}\text { Training } \\
\text { Type }\end{array}$ & $\boldsymbol{n}$ & Mean & SD & Min & Max & LCL & UCL \\
\hline Blended & 95 & $4,013.45$ & $22,158.07$ & 0.00 & $214,999.61$ & 0.00 & $8,527.28$ \\
\hline BTW & 42 & $2,738.02$ & $4,127.38$ & 0.00 & $22,222.00$ & $1,454.84$ & $4,024.21$ \\
\hline Simulator & 15 & 457.31 & 621.10 & 0.00 & $2,096.60$ & 113.36 & 801.27 \\
\hline
\end{tabular}

Note: Values in US Dollars (\$).

\section{Average Cost of Crashes per Thousand Miles Driven}

The cost of crashes per thousand miles driven was examined for differences across training types. As in the examination of cost per crash, large variations in the number of cases across training types and the number of censored cases (drivers who terminated employment prior to the end of data collection) present in the data, an ANOVA did not provide any more insight than the comparisons of means and confidence intervals. This calculation was performed only for drivers with both a crash recorded and mileage log data available $(n=136)$. Thus, drivers with no crashes and/or without driving log data were excluded from this analysis. Additionally, the one crash viewed as an outlier (costing more than $\$ 200,000$ ) was excluded from the analysis as this would unduly influence the comparison between training types. Note that some zero-cost $(\$ 0.00)$ crashes were recorded in the data set, however these events were included in the analysis and 
resulted in a minimum observed cost of crashes per thousand miles driven value of zero dollars (\$0.00). These crashes were left in the analysis due to their influence on driver tenure (many carriers’ policies allow drivers a specific number of crash events before being terminated). Drivers receiving simulator training had a lower average cost per thousand miles in terms of crashes (approximately \$12) when compared to drivers in both the BTW and blended groups (approximately $\$ 81$ and $\$ 49$, respectively). Table 67 shows the descriptive statistics for the average cost of crashes per thousand miles across each training type.

Table 67. Descriptive Statistics for Cost of Crashes per Thousand Miles Driven

\begin{tabular}{|l|r|r|r|r|r|r|r|}
\hline $\begin{array}{l}\text { Training } \\
\text { Type }\end{array}$ & $\boldsymbol{N}$ & Mean & \multicolumn{1}{c|}{$\boldsymbol{S D}$} & Min & Max & \multicolumn{1}{c|}{ LCL } & \multicolumn{1}{c|}{ UCL } \\
\hline Blended & 87 & 49.38 & 97.80 & 0.00 & 560.85 & 28.54 & 70.22 \\
\hline BTW & 35 & 81.16 & 121.24 & 0.00 & 412.55 & 39.51 & 122.80 \\
\hline Simulator & 14 & 11.62 & 17.10 & 0.00 & 59.56 & 1.75 & 21.49 \\
\hline
\end{tabular}

Note: Values in US Dollars (\$).

Summary of Results: Training, safety, and mileage data from 2,459 drivers were provided by Carrier A to the research team. Due to missing cases across data sets (e.g., missing mileage data from drivers), some cases were filtered from specific analyses (as noted above). A survival/failure analysis approach was chosen as the general analytic strategy. This approach allowed for the ability to account for cases dropped during data collection, prediction of time-toevent, and ability to produce hazard ratios illustrating differences between conditions.

Survival/failure analyses were conducted for driver tenure (in terms of time of tenure and mileage during tenure) and crash involvement (in terms of time to crash). Limitations with the data available (e.g., lack of descriptive categorization regarding crash involvement) prohibited more in-depth analyses of certain aspects of driver tenure and crash involvement; therefore, these results and conclusions should be considered in light of these limitations.

Driver tenure appeared to be positively affected by truck simulator-based driver training. Both simulator- and blended-trained drivers had significantly longer durations of tenure (as measured in days) compared to BTW-trained drivers. There was no significant difference between the simulator and blended groups with respect to driver tenure. The same result was found when tenure was measured as miles driven rather than days. Both simulator- and blended-trained drivers had logged significantly more miles during their tenure as compared to BTW-trained drivers. Additionally, simulator-trained drivers logged a significantly greater number of miles during their tenure of employment than did blended-trained drivers.

Based on the analyses, it appears that both simulator and blended training had a positive influence on the tenure of drivers (both in days and miles logged) when compared to BTW training. However, it was not possible to ascertain if either simulator or blended training were superior to BTW training with respect to driver tenure. Other explanatory factors for termination of employment (e.g., crash, at-risk driving behaviors, not following carrier polices, resignation) were unknown and may have influenced driver tenure.

Crash involvement, accepting any event logged by the Carrier A as an "accident” regardless of cost, was examined as a function of days until the first crash. Overall, the majority of drivers 
(93.82 percent of drivers across all training types) had zero crashes recorded during the measurement period. Hazard ratios calculated during the survival/failure analysis revealed that drivers in both the simulator and blended groups had a higher likelihood of a crash earlier in their tenure as compared to BTW drivers. Although this difference was statistically significant, the pragmatic effect of the difference was negligible due to the small difference between groups. Blended drivers traveled an average of 48 days prior to crash, compared to 46 and 48 days for BTW and simulator drivers, respectively. Thus, the statistically significant difference in time to crash was only 2 days.

The cost per crash was analyzed with descriptive statistics due to a severe imbalance in the number of observations across different training types (over 6 times as many drivers in the blended group as compared to the simulator group). Drivers receiving simulator training had the lowest overall cost per crash $(M=\$ 457.31, S D=621.10)$ as compared to both the blended $(M=\$ 4,013.45, S D=22,158.07)$ and BTW drivers $(M=\$ 2,738.02, S D=4,127.38)$. Although the mean and standard deviation for the blended drivers was adversely influenced by the presence of an outlier (a single crash of over \$200,0000 in cost), the mean cost per crash of simulator drivers was eight and six times lower than blended and BTW drivers, respectively.

Crash involvement was also examined in terms of the cost of crashes per thousand miles traveled to control for exposure. The resultant analysis demonstrated that drivers in the simulator group had the lowest overall mean cost per crash per thousand miles traveled $(M=\$ 11.62$, $S D=17.10)$, followed by blended $(M=\$ 49.38, S D=97.80)$ and BTW drivers $(M=\$ 81.16$, $S D=121.24)$, respectively. Although mean cost per crash and cost of crashes per thousand miles driven was not a true descriptor of crash severity, it may be viewed as a reasonable surrogate for crash severity. It appears that simulation training had a positive effect on the severity of a crash (as measured in cost). Again, this result should be viewed with caution. Any number of variables could have contributed to the occurrence of a single crash and its associated cost. The limited data available for the present analysis precluded the assumption that simulator training was superior to blended or BTW training without knowing the circumstances of the event .

In general, the truck simulator-training program at Carrier A appeared to be beneficial (Table 68). Given the caveats above, the truck simulator-training program appears to be associated with increases in driver tenure and reductions in crash severity and involvement. These results appear to support the findings of researchers (Brock et al., 2001) who have noted the safety and efficiency benefits of truck simulator-training for commercial drivers. 
Table 68. Major Findings from Carrier A Effectiveness Data

\begin{tabular}{|l|l|}
\hline \multicolumn{1}{|c|}{ Measure } & \multicolumn{1}{c|}{ Findings } \\
\hline Driver Tenure & $\begin{array}{l}\text { Simulator-trained drivers were 34 percent less likely to } \\
\text { leave employment during the observation period }\end{array}$ \\
\hline Miles Logged during Tenure & $\begin{array}{l}\text { Simulator trained drivers were 44 percent more likely } \\
\text { to log more miles during the observation period }\end{array}$ \\
\hline Crash Rate & $\begin{array}{l}\text { No apparent difference between simulator- and BTW- } \\
\text { trained drivers during the observation period }\end{array}$ \\
\hline Time to First Crash & $\begin{array}{l}\text { Simulator-trained drivers more likely to be involved in a } \\
\text { crash before BTW-trained drivers during the } \\
\text { observation period; difference was 2 days }\end{array}$ \\
\hline Cost per Crash & $\begin{array}{l}\text { Simulator-trained drivers had a lower cost per crash } \\
\text { during the observation period }\end{array}$ \\
\hline $\begin{array}{l}\text { Crash Cost per 1,000 Miles } \\
\text { Driven }\end{array}$ & $\begin{array}{l}\text { Simulator-trained drivers had a lower cost per crash } \\
\text { per 1,000 miles driven during the observation period }\end{array}$ \\
\hline
\end{tabular}

\subsubsection{Carrier B}

Although quantitative data of driver performance was not available from Carrier B, information regarding the effectiveness of simulator-based training was obtained during interviews with carrier officials. Thus, the following summary of the effectiveness of Carrier B's truck simulator training program was developed through these anecdotal conversations with Carrier B officials.

Carrier B measured the effectiveness of their blended driving training program (during which approximately 20 percent of driving time was completed in the truck simulator and the remaining time spent in BTW training) through multiple methods. During their pilot testing (initial implementation) of blended driver training, the carrier compared efficiency and effectiveness of the new blended driver training against the same measures at their other training facilities which had not included simulators in their driver training program. During this pilot testing, approximately 1,600 drivers were measured to record the efficiency and effectiveness of the blended training approach.

The primary efficiency measure in the truck simulation training program was cycle time. This was a straightforward measure of the time needed to send a driver through the training program and included the time from the beginning of the training program (typically the hire date of the trainee) through final testing. Carrier B observed significant reductions in training cycle time for drivers completing the blended training program as compared to their existing BTW training. Although the exact magnitude of this effect was not provided, the implications of any real reduction in cycle time would yield cost savings for larger carriers due to the number of drivers involved. Effectiveness measures used by Carrier B included safety (factors such as collisions and driving safety violations), trainee dropout and turnover rates, and the number of days until the trainee was working in a revenue-generating truck. In the 4 years following the implementation of the blended training approach at Carrier B, the carrier reported cost savings and safety improvements beyond those obtained with BTW training. These findings of increased efficiency and effectiveness of training led Carrier B to widen the implementation of their blended training approach to include all entry-level drivers. 
Additionally, the carrier integrated the truck simulators in bi-annual safety training. During the spring training event, all drivers complete an in-house safety refresher training that includes truck simulator-based training. During the winter safety training session, drivers either complete an online course (if they are deemed to have a lower safety risk) or return to the in-house training program for targeted training with the truck simulator (if they are deemed to have a higher safety risk). Carrier B expects to increase the use of truck simulator technology (and e-learning online training) for refresher training over the next several years.

\subsection{DRIVERS’ PERCEPTIONS OF SIMULATOR-BASED TRAINING}

\subsubsection{Introduction}

Two focus groups (one at each carrier) were conducted to examine trainees’ subjective experiences with truck simulation-based training. The trainees from the two carriers provided insightful and sometimes contrasting information, as the two different carriers held different approaches to truck simulation-based training. The approach to conducting each focus group followed a three-step process:

Identify and recruit target participant population for the focus group.

Conduct focus group.

Transcribe and analyze the focus group findings.

Each of these steps is described below.

\subsubsection{Method}

\subsubsection{Participant Recruitment}

The purpose of this portion of the study was to document how existing carriers operate truck simulator training programs. Therefore, only students currently enrolled in, or who had recently completed, a program were eligible to participate in the focus groups. Truck simulator training programs were defined as a driver training program, including entry-level or experienced driver training, which involved the use of a truck simulator as a mandatory part of training.

Specific participation criteria were:

Hold a valid Class A CDL.

Recently (within the past 6 months) attended the carrier's simulator training program. Note that the requirement for holding a Class A CDL resulted in all participants being at least 21 years of age.

Participants were recruited by company officials at the conclusion of their truck simulator training class or session. Potential participants were given recruitment flyers listing the details and purpose of the focus group, as well as the requirements for participation, type of compensation, and time requirements. 


\subsubsection{Participant Demographics}

Two focus groups were conducted. The first was conducted at Carrier A on October 17, 2008 $(n=5)$, while the second was held at Carrier B on November 17, $2008(n=12)$. Both focus groups were conducted in private classrooms at terminal locations with only the participants and moderator(s) present. All participants had recent (that is, within the previous 6 months) experience with their carrier's truck simulation-based training program.

A total of 16 males and one female participated in the focus groups $(M=45$ years old, $S D=7.7)$. On average, participants had 12.6 years experience driving a CMV $(S D=9.4)$. A majority of the participants $(n=11)$ held at least one endorsement on their license. Five of these 11 drivers held multiple endorsements. The most common endorsement was Doubles/Triples (endorsement "T", $n=6$ ), Hazardous Materials (endorsement “ $H$ ”, $n=6$ ), and Tank Vehicle (endorsement “N”, $n=$ 6) endorsements. Three drivers held Passenger ("P") endorsements. All participants held a valid Class A CDL and reported English as their preferred language (for both written and verbal communication).

Three of the five participants from Carrier A identified themselves as African-American (the other two participants were Caucasian). At Carrier B, five participants identified themselves as African-American, five as Caucasian, and two as Native-American. Three of the 5 participants from Carrier A held endorsements. Of these, one held a double/triple trailer endorsement, one tank endorsement, and one tank, double/triple trailer, hazardous materials, and passenger endorsements. Eight of the 12 participants from Carrier B held endorsements. Of these, two participants held hazardous materials endorsements, one hazardous materials and passenger endorsements, one double/triple trailer and passenger endorsements, and the remaining participants held a tank endorsement. No participant was a member of a professional or trade union. Table 69 presents participants' self-reported background demographics, by carrier (including moving violation and crash involvement over the past 36 months).

Table 69. Participant Demographic Information

\begin{tabular}{|c|c|c|c|c|c|c|c|}
\hline Carrier & Age & Sex & $\begin{array}{c}\text { CMV Driving } \\
\text { Experience } \\
\text { (SD) }\end{array}$ & $\begin{array}{c}\text { Tenure with } \\
\text { Carrier (SD) }\end{array}$ & $\begin{array}{c}\text { Moving } \\
\text { Violations } \\
\text { (SD) }\end{array}$ & $\begin{array}{c}\text { Crashes } \\
\text { (all types) } \\
\text { (SD) }\end{array}$ & $\begin{array}{c}\text { Crashes } \\
\text { (at-fault) } \\
\text { (SD) }\end{array}$ \\
\hline Carrier A & $\begin{array}{c}46.8 \\
(12.2)\end{array}$ & $\begin{array}{c}5 \text { males, } \\
0 \text { females }\end{array}$ & $13.8(8.1)$ & $8.7(6.7)$ & $0.20(0.45)$ & $0.40(0.55)$ & $0.40(0.55)$ \\
\hline Carrier B & $\begin{array}{c}44.3 \\
(5.4)\end{array}$ & $\begin{array}{c}11 \text { males, } \\
1 \text { female }\end{array}$ & $12.2(10.2)$ & $\begin{array}{c}\text { all newly } \\
\text { hired }\end{array}$ & $0.33(0.49)$ & $0.08(0.29)$ & $0.00(0.00)$ \\
\hline
\end{tabular}

Note that Age, Driving Experience, and Tenure with Carrier are presented in years. Standard deviations are presented in parentheses following means. Moving Violations and Crashes represent only violations and crashes during CMV driving. All information was self-reported.

\subsubsection{Focus Group Procedure}

The two focus groups were held on different days at conference rooms at each carrier's training facility. Each focus group lasted 2 hours. Participants were greeted and escorted to the focus group area. As described above, 5 male participants participated in the focus group at Carrier A, while 12 participants (11 males and 1 female) participated in the focus group at Carrier $\mathrm{B}$. 
Focus group participants were given an informed consent form and were given time to read the document and ask questions regarding the form prior to signing. Two copies of the document were signed, one for the research team's records and a second copy for each participant. Participants were also reminded that the focus group session would be audio-recorded and that they may refuse to answer any question or leave the focus group at any time without penalty.

Both focus group meetings followed the protocol outlined in appendix N. The first topic covered was a discussion of the focus group procedure and logistics (including the form, why the focus group was being conducted, issues of participant confidentiality, and the rules of the meeting). This discussion lasted approximately 10 minutes. The remainder of the focus group was divided into six topics that covered the following:

Benefits of Truck Simulators. Participants were asked who would benefit most from a truck simulator training program. This topic was covered as a brainstorming activity during each group, with responses written on the whiteboard.

Differences in Simulator versus BTW Training. Participants were asked what differences were present between traditional methods of training, using a real tractor-trailer, and simulator-based training. This topic was covered as a whiteboard activity, with parallel lists of similarities and differences left visible on the whiteboard through the end of Topic 6.

Changes in Driver Training from the use of Simulators. Participants were asked to explore if the use of truck simulators altered driver training and, if so, how it affects driver training.

The Role of Simulation in Training. Participants were asked what the proper use, or role, of a truck simulator was in driver training.

Transfer of Training. Participants were asked what level of transfer of training would occur between truck simulator training and real-world driving.

Best Practices and Areas to Improve. Participants were asked to describe the benefits and disbenefits of the truck simulator training (i.e., what would they keep or change).

At the conclusion of the last topic, participants were thanked for their participation and given a chance to pose questions regarding their participation. Participants were then asked to fill out a brief form with the name and address they wished to provide for payment. Participants were compensated \$25 per hour for their participation, thus all participants received a total of \$50.

\subsubsection{Transcribe and Analyze Data}

The focus group meetings were audio-recorded as a supplement to researcher notes and written responses provided by participants. As a precaution for participant confidentiality, no names or specifically identifying information were used during the focus group meetings. Additionally, transcripts produced from the recordings did not include any identifying information for any participant. Recordings were erased after the analysis was completed. 


\subsubsection{Results}

The results of the focus group meetings, including written and oral responses to questions, were analyzed. The results, provided by each major topic, are described below.

\subsubsection{Topic 1: Benefits of Truck Simulators}

The first question asked by the moderator was "Who would benefit most from a truck simulator training program?” The question was phrased as a brainstorming question, with participants asked to quickly provide the "first to mind" responses while the moderator wrote their responses on the whiteboard. This question was intended to serve as an introductory, warm-up question for participants and allow them to feel confident discussing the topics raised during the focus group meeting. However, the findings also illustrate driver beliefs regarding truck simulator training.

A total of 12 participants responded to the question. Although no specific category of response was solicited from participants, their responses were self-limited to basic categories. Five participants responded that all drivers would benefit equally from simulator-based training. The second most frequent response was entry-level drivers, with four participants responding that socalled "rookie drivers" (drivers with a recently obtained Class A CDL) were most likely to benefit from truck simulator training. Safety categories of complacent drivers, drivers who recently experienced a crash, and tank-endorsement drivers were proposed by one participant. Results of the frequencies of all responses to this topic are summarized in Figure 44.

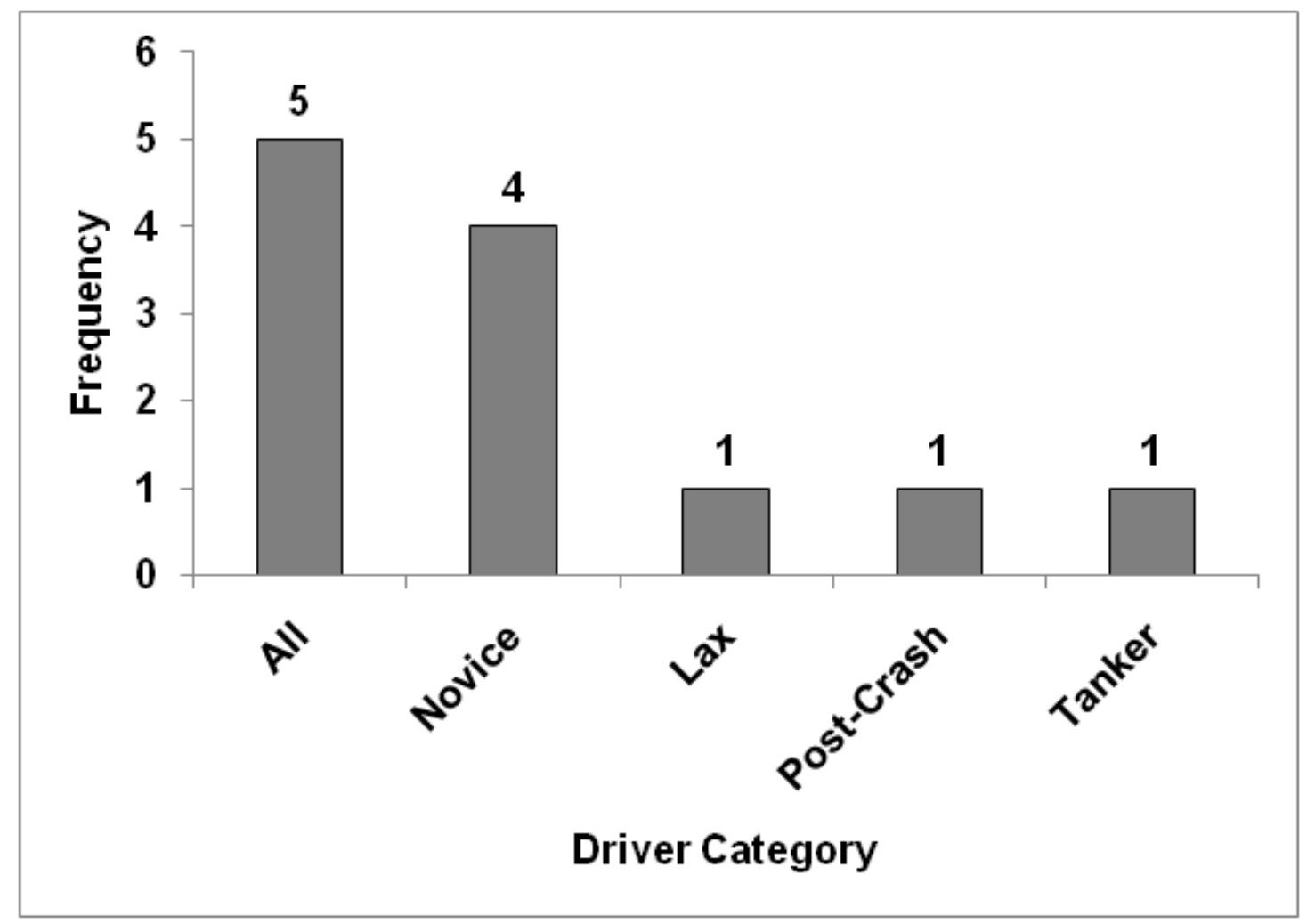

Figure 44. Graph. Frequency of participant responses to "Who would benefit most from a truck simulator training program?" 
Almost all participants believed that all drivers would benefit from training in a truck simulator. However, the majority of participants identified entry-level drivers as likely to obtain the most benefit from truck simulator training. These participants stated the truck simulator allowed entrylevel drivers the opportunity to experience situations that would be dangerous in the real world, without the associated risk and costs of an adverse event.

A small number of participants identified drivers who recently experienced a crash as likely to obtain the most benefits in truck simulator training. One participant identified tanker drivers, as he believed the drivers of tanker-trailer vehicles would benefit from controlled practice. One participant identified lax drivers (drivers who develop an unconcerned attitude towards driving safety). This participant described the lax driver as one who thought, "Yeah, I know I can do this." This led other participants to agree that introducing rare or challenging driving tasks during truck simulator training sessions would serve as a valuable skill check for lax drivers and, as one participant stated, "bring the driver back to reality." Another participant indicated that post-crash drivers receiving truck simulator training would serve as a valuable skills-check prior to resuming professional driving and would allow the driver to be reintroduced to driving in a less stressful and physically demanding manner. One participant believed tanker drivers would benefit the most from truck simulator training, as the simulation would provide a safer environment for training.

In follow-up discussions, many participants stated that veteran drivers would obtain benefits from truck simulator-based refresher training (albeit not to the level of benefit entry-level drivers could obtain). This was especially true for training responses to emergency maneuvers, such as steering during tire blowouts, skids, and driving in adverse weather conditions. Participants believed exposure to these situations in the truck simulator increased driver confidence and helped drivers develop a plan for how to proceed in challenging conditions.

In addition, some participants (from Carrier A) believed that over-the-road (OTR) drivers would obtain the greatest benefit from truck simulator training. These participants stated that their truck simulator training scenario consisted of interstate/highway driving, so these OTR drivers could experience a more accurate simulation of what they were likely to experience while driving. Carrier B participants did not believe drivers with different haul or route types would obtain different benefits from truck simulator-based training. The majority of participants indicated that the opportunity to experience simulated scenarios with a high degree of similarity to their actual driving experience would yield the greatest benefits.

When asked which drivers would be the least likely to benefit from truck simulator-based training, most participants believed drivers who were either complacent or assigned to truck simulation training (i.e., not by their choice) were the least likely to benefit. Participants stated that drivers who were forced to attend truck simulator-based training were likely to have an attitude of resistance towards training. Likewise, participants indicated that complacent drivers were likely to believe they would not benefit from training, stating, "This doesn't apply to me, I've been driving for 20 years".

There was also discussion about veteran/experienced and older drivers. Most participants from the Carrier A focus group believed these drivers were less likely to benefit because of their CMV driving experience ("they already know it") or a resistance to novel or new training techniques. 
One participant stated that trying to get experienced drivers to work with a truck driving simulator was like "teaching an old dog new tricks." Participants also believed that a driver's age factored into their resistance toward truck driving simulator training, especially since many older drivers had limited experience with computers. Some participants also noted that older drivers had trouble adapting to new electronics or technology, and that their response to simulator-based training would likely prompt a similar resistance.

Participants disagreed about the magnitude of benefit received during specific driving scenarios using a truck simulator. Some participants indicated that the scenarios were not realistic enough to benefit a driver already operating a real truck. However, other participants believed the exposure to the situation in a truck simulator would create increased awareness during a similar situation in a real truck. Although some participants believed that training for specific scenarios (such as loss of brakes, snow/ice, etc.) would not be beneficial, most participants believed exposure to these situations via the truck simulator would be helpful in assessing the likelihood of a dangerous scenario. One participant said, "It's better that I know what to do, whether or not it is realistic enough.” Many participants indicated the truck simulator was better at training their situational awareness.

Summary: In summary, participants believed the majority of drivers would benefit from simulator-based training. Moreover, many participants believed all drivers would benefit, especially entry-level drivers.

\subsubsection{Topic 2: Differences in Simulator versus BTW Training}

Participants were asked to discuss similarities and differences in a truck simulator compared to a real vehicle (BTW training). A total of 15 participants provided responses to the question.

Participants privately listed their top similarities and differences on a notepad and provided their top response to the moderator. Participant responses were listed on a whiteboard (which remained visible through the end of the focus group). Interestingly, both carriers used similar truck simulator equipment and software; however, they differed in the implementation of their respective truck simulator training programs (Carrier A used their simulator for refresher and targeted training, while Carrier B used their simulator for all new hires, entry-level drivers, and refresher training). These similarities and differences in the implementation of simulator-based training are reflected in participant responses.

The majority of participants indicated that the greatest similarity between simulated and BTW driving were vehicle reactions (or the physical reactions of the simulated versus real vehicle) and the equipment in each vehicle (each identified by four participants). The majority of participants indicated that the greatest difference between simulated and BTW driving was the safety of a truck simulator compared to BTW training (identified by five participants).

Some responses by participants were identified as similarities, while other participants noted the same response as a difference between simulator and BTW driving. This occurred in four of the participant responses:

Vehicle reactions.

Mirrors. 
The overall feel of driving (subjective realism).

Steering.

Figure 45 displays the frequency of participant responses noted as both a similarity and difference when comparing simulator and BTW driving. As shown in Figure 45, the greatest disparity was in vehicle reactions (four participants identified as a similarity, two as a difference) and feel of driving (one participant identified as a similarity, three as a difference). Two participants identified mirrors as a primary difference, while one identified it as a similarity.

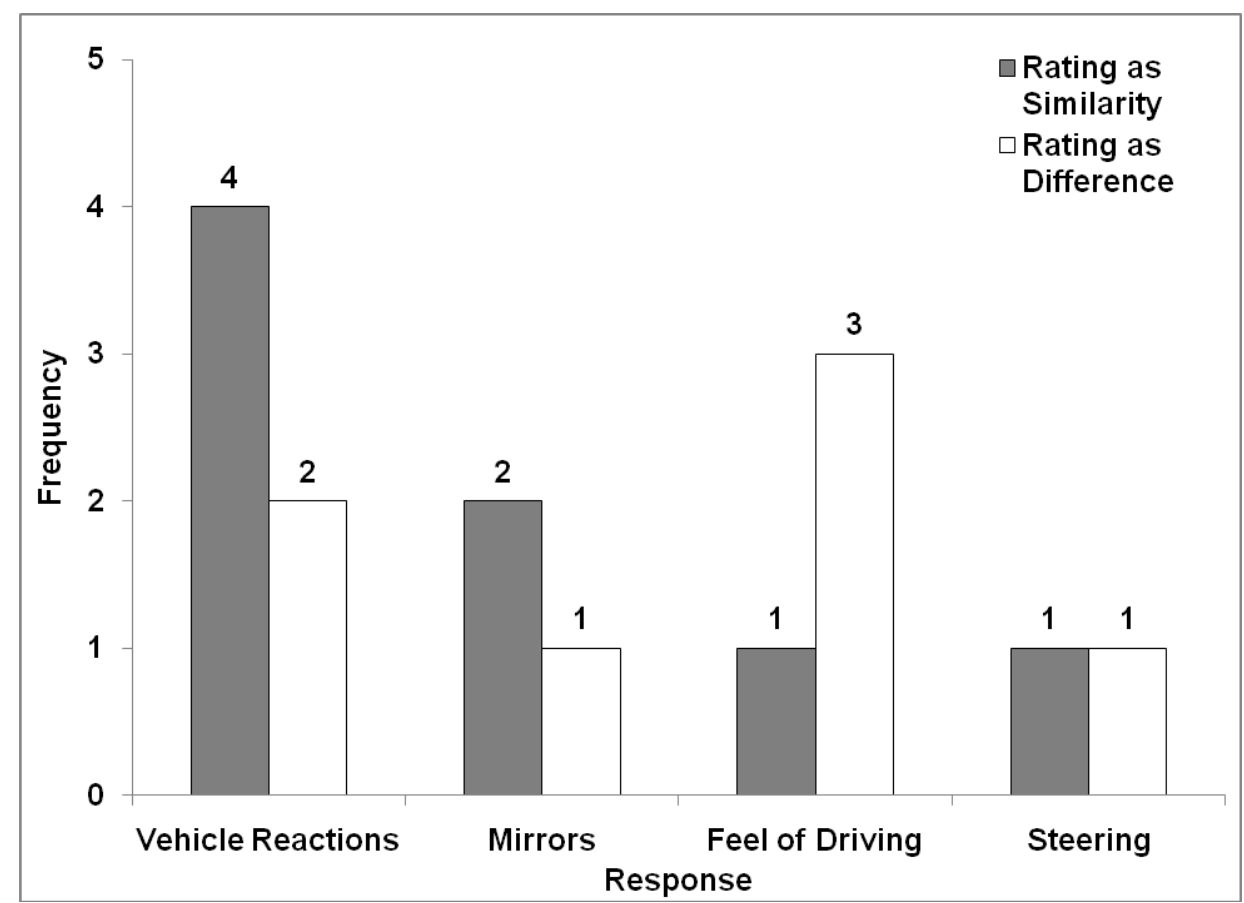

Figure 45. Graph. Comparison of participant responses noted as both a similarity and difference in simulator and BTW driving.

Upon follow-up questioning, many participants believed that the reactions of the simulated vehicle were very similar to those of a real truck. Participants indicated the accuracy of the engine speed and shifting interaction while ascending and descending steep grades in the simulator mimicked those in a real truck. Other similarities between the truck simulator and real truck noted by participants included dealing with in-cab vibrations, monitoring engine speed, handling tire blowouts, and mirrors (though some participants indicated the truck simulator mirrors were more effective than mirrors in a real truck). These participants believed the simulator mirrors should be adjustable to reflect a different visual field-of-view. Additionally, it was believed the truck simulator mirrors should include blind spots similar to those of a real truck.

Participants also discussed the discrepancies in driver mindset during simulator training compared to BTW training. Participants believed the truck simulator fostered a mindset that their interactions in the truck simulator were not real, thus, their actions within the simulated environment would have little effect on anything. Certain aspects of the simulated environment encourage this sensation, such as the condensed nature of scenarios (with simulated incidents and 
events occurring more frequently than in the real world) and differences in the simulated truck's steering and braking systems. Additionally, while the greater safety of truck simulator training was identified as a positive aspect, some participants noted that the lack of direct consequences fostered negative attitude towards the simulator (seeing the simulator as a game). A list of participant responses regarding similarities and differences in simulator and BTW driving are shown in Table 70.

Table 70. Frequency of Participant Responses Regarding Similarities and Differences in Simulator and BTW Driving

\begin{tabular}{|l|c|c|}
\hline Participant Response & $\begin{array}{c}\text { Rated as } \\
\text { Greatest } \\
\text { Similarity }\end{array}$ & $\begin{array}{c}\text { Rated as } \\
\text { Greatest } \\
\text { Difference }\end{array}$ \\
\hline Braking & 0 & 2 \\
\hline Collision Realism & 1 & 0 \\
\hline Driver Mindset & 0 & 2 \\
\hline Equipment/Instrumentation & 1 & 0 \\
\hline Feel of Driving & 1 & 3 \\
\hline Learning Process & 1 & 0 \\
\hline Mirrors & 2 & 1 \\
\hline Non-Realistic Timeline & 0 & 1 \\
\hline Overall Equipment & 4 & 0 \\
\hline Repeatable Scenarios & 0 & 1 \\
\hline Safety & 0 & 5 \\
\hline Shifting & 1 & 0 \\
\hline Sounds & 2 & 0 \\
\hline Steering & 1 & 1 \\
\hline Vehicle Reactions & 4 & 2 \\
\hline
\end{tabular}

During the discussion, a participant described the differences between the truck simulator and real trucks as being complementary (a contention supported by other participants). This participant indicated his carrier (Carrier A) had trainees practice the maneuver in the truck simulator prior to practicing the same maneuver in a real truck. The participant believed it was beneficial to experience the driving situation in the truck simulator followed by the real truck.

One participant believed the differences between the truck simulator and the real truck were too significant to depend on the truck simulator as the sole training approach. The lack of consequences and accurate motion cues in the truck simulator were the primary differences that would need to be addressed prior to relying on a truck simulator as the primary training vehicle. This participant compared the truck simulator to an arcade game, stating, "How many times have you been to the arcade and driven a racecar? Do you think you could get in a real racecar and race against professional drivers and do very good? You might, but it’s not likely.”

Summary: The physical reactions and subjective driving feel were cited as both a similarity and difference of the simulator as compared to BTW driving. Driver attitude towards simulator-based 
training was identified as a barrier to successful training. However, the similarities between the operation of the simulator and a truck were identified as beneficial to simulator-based training.

\subsubsection{Topic 3: Changes in Driver Training from the use of Simulators}

Participants were asked by the moderator to indicate if the use of a truck simulator changed their training process as compared to traditional BTW training. Participants responded verbally, with follow-up questions from the moderator. Table 71 displays the frequency of participants' responses to the question "Does use of a truck simulator change the driver training process as compared to traditional BTW training?” All 17 participants who responded believed that training in a simulator changed the process of training when compared to traditional BTW training. Following this response, the moderator inquired about the specific changes in the training process resulting from simulator training.

Table 71. Frequency and Percentage of Participants' Response to "Does use of a Truck Simulator Change the Driver Training Process as Compared to Traditional BTW Training?"

\begin{tabular}{|l|c|c|}
\hline Response & Frequency & Percentage \\
\hline Yes & 17 & $100 \%$ \\
\hline No & 0 & $0 \%$ \\
\hline
\end{tabular}

Almost all participants noted the importance in the physics of the simulation to match a real truck. Although subtle, the truck simulator's lack of simulated weight and momentum-shifting during the simulation was a limiting factor. Participants believed this restricted the ability to train drivers in recognizing subtle driving cues, such as being aware of impending skids or learning the implications in shifting while towing heavy loads. One participant stated that shifting was the most important behavior a new driver needed to learn; thus, the truck simulator was not providing an accurate re-creation of the shifting task beyond basic skills (due to the lack of interaction between shifting and weight of the towed load). In addition, some participants believed the feeling of being observed was more salient in the truck simulator compared to a real truck.

The lack of direct safety consequences within the simulated environment was related to a number of modifications to the driver training curriculum identified by participants. Participants believed this allowed drivers to train for many rare or dangerous scenarios (e.g., steering through tire blowouts, skids) which could not be easily accomplished in BTW training. Participants did not feel that training for these types of events was occurring in BTW training, and the changes to their training to include these rare and dangerous scenarios was beneficial. While participants believed the lack of casualties and equipment damage allowed exposure to events which would be difficult or impossible in real vehicles, they also believed the simulated environment heighted drivers' overall safety awareness. Participants believed this allowed drivers to experience, and be aware of, their own level of driving safety behaviors. Furthermore, participants believed this allowed for training of rare safety-critical events; training for rare safety-critical events was not believed to be possible to accomplish in BTW training.

The ability to review and play back prior driving behaviors and performance was noted by participants as one of the critical components in simulator truck training. Participants stated they were acutely aware of being recorded and monitored within the truck simulator; thus, some 
participants indicated that this led to more careful and reserved driving in the truck simulator than in a real truck. However, these participants also believed that the ability to pause and replay driving behavior and performance in the truck simulator (a simulator feature used as part of the training both groups experienced) was a positive training experience. Participants viewed the video replays as an opportunity to review a mistake and learn the root cause (rather than merely being informed of a mistake, as in the real truck), as well as provide a way to correct the problem.

Summary: All participants believed the use of simulators changed the trainer's approach to driver training. The simulator's lack of many subtle cues (that are present in BTW driving) was identified as a barrier to driver training. However, the lack of safety consequences for mistakes during simulator driving was identified as a positive aspect to simulator-based training.

\subsubsection{Topic 4: The Role of Simulators in Training}

Participants were asked to speculate on the role of truck simulators in CMV driver training. This question was phrased by the moderator so participants could reflect on the overall process of CMV driver training and provide feedback as to where the truck driving simulator would best be utilized. Responses were solicited from each participant with follow-up questions from the moderator.

Figure 46 displays the percentage of responses to the question "What is the ideal time for truck simulator-based training?” Most participants (77 percent) indicated the truck simulator would best be used prior to exposure to BTW driving. A smaller percentage of drivers (23 percent) believed some form of BTW training should occur prior to the use of truck simulators. However, follow-up questions from the moderator revealed some differences in participants' views of when truck simulator training should be implemented.

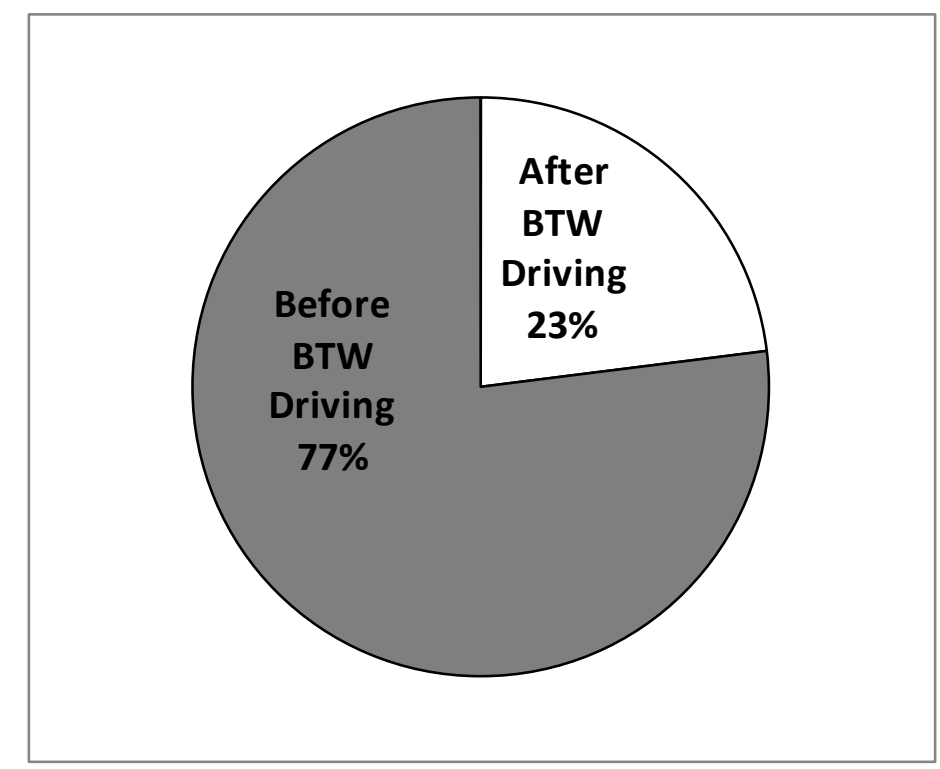

Figure 46. Chart. Percentage of participants' responses to "What is the ideal time for truck simulator-based training?" 
Follow-up questioning revealed that most participants believed the truck simulator should serve as a device to improve situational awareness and safety-conscious behavior. Many of these participants believed the truck simulator's appropriate role was that of a skills-development and maintenance tool. These participants believed the simulator could be used to train basic skills, including shifting, mirror use, and lane-holding skills, or could be integrated into their current Smith System training (a commercial defensive driving training course for professional drivers). Specific participant responses provided during the discussion are provided in Table 72.

Table 72. Participant Comments Regarding the Ideal Use of Truck Simulators in Entry-level CMV Driver Training

\begin{tabular}{|l|l|}
\hline \multicolumn{1}{|c|}{ Response } & \multicolumn{1}{c|}{ Comment } \\
\hline After BTW & Use specifically for training rare situations. \\
\hline After BTW & $\begin{array}{l}\text { Emergency maneuvers only. Cannot be used for training } \\
\text { backing. }\end{array}$ \\
\hline After BTW & After CDL testing, use for awareness training. \\
\hline After BTW & $\begin{array}{l}\text { If driver has already driven a truck, then simulator. Otherwise, } \\
\text { real truck first with truck simulator training afterwards }\end{array}$ \\
\hline Before BTW & $\begin{array}{l}\text { Use for training situational awareness and recovery from } \\
\text { dangerous situations. }\end{array}$ \\
\hline Before BTW & $\begin{array}{l}\text { Use for training situational awareness and procedural tasks } \\
\text { (mirror checks, lane discipline). Helps reduce anxiety in real } \\
\text { trucks. }\end{array}$ \\
\hline Before BTW & Use for training basics, such as shifting. \\
\hline Before BTW & $\begin{array}{l}\text { Use for teaching situational awareness of common crash } \\
\text { scenarios (automotive collisions during right turns) }\end{array}$ \\
\hline Before BTW & Use when introducing Smith System. \\
\hline Before BTW & Use for situational awareness training. \\
\hline Before BTW & Use when introducing Smith System. \\
\hline Before BTW & Use when introducing Smith System. \\
\hline Before BTW & Use for preventing common entry-level driver collisions. \\
\hline
\end{tabular}

In addition to using the truck simulator in entry-level driver training, some participants believed the truck simulator could play a prominent role in retraining drivers who experienced a crash or other type of safety event. Some of these participants indicated that training in a truck simulator following a crash, but prior to resuming on-road driving, would allow the driver to "refresh [his or her skills] without a lot of [physical] impact.” However, these same participants indicated that refresher training following a safety incident was the reason many participants expressed displeasure with the carrier's policy of mandatory safety training following a crash. Many participants indicated that the combination of mandatory training (regardless of fault) —with reduced pay during that training — was a form of punishment. Participants indicated that better pay, pre-training briefings, and allowing drivers to request specific types of training could improve the integration of truck simulators in CMV refresher training programs.

Most participants believed the simulator would be extremely useful as a regularly scheduled training device. When the moderator asked participants to indicate how often truck simulator refresher training should occur, one participant indicated that biannual training would be most 
appropriate. The majority of participants suggested that refresher training should be performed once every two years. However, one participant indicated that refresher training should occur once every four years (or during a driver's license renewal). This participant viewed training as an inconvenience and something that prevented the participant from making a revenuegenerating delivery. Upon further discussion, a consensus was reached among participants that refresher training should be conducted biannually, in order to break the monotonous mindset of drivers.

The moderator also asked participants to indicate the most important training scenario to be used during refresher training (regardless if it was not currently used). The majority of participants indicated that the most important training scenario involved a heavy freeway traffic environment with bad weather (as they believed it was the most common hazardous driving condition encountered). Other suggestions from participants included steering during tire blowouts, loss of brakes resulting in the use of a runaway truck ramp, and rehearsals for post-crash procedures. Participants agreed that simulator training should be progressive, building on previous knowledge and exercises, and not structured as discrete learning objectives.

Many participants believed that, in addition to regularly scheduled refresher training, truck simulators should be made available to drivers (i.e., on-demand). One participant stated he would be willing to pay for this type of simulator access. All participants agreed that on-demand truck simulator training would be a beneficial resource, especially if there were multiple driving scenarios available, including:

Seasonal driving training.

Handling off-road (runaway truck) ramps.

Animal strikes.

Adverse weather conditions (fog, rain, snow).

Construction areas.

Handling sudden/unexpected vehicle failures.

Rehearsal drives specifically tailored for different regions.

Participants indicated that on-demand training could be a useful training tool as it would allow each driver to experience the consequences of different driving maneuvers and environments. Likewise, most participants indicated a need for some form of scoring during the scenarios, allowing them to self-monitor their behavior. Participants cautioned that any scoring system provided must be confidential to prevent other drivers and/or managers from viewing their results or the scenarios they selected for training. One participant commented, "We have a lot of downtime, it would give us something to do and we'll be learning at the same time ... who wouldn't want to get on the simulator?”

Summary: The majority of participants believe simulator-based training should occur prior to exposure to BTW driving. Also, training for situational awareness and defensive driving was viewed by most participants as an appropriate role for the simulator. 


\subsubsection{Topic 5: Transfer of Training}

Participants were asked if they believed the information they learned in the truck simulator would carry over (transfer) to real-world driving. Responses were sought from each participant and follow-up questions were asked by the moderator. Table 73 shows the frequency and percent of participants' responses to the question, "Does the information you learn in the truck simulator carry over to real-world driving?” All participants believed some level of transfer-of-training would occur, with the majority (63 percent) believing the specific training received in the simulator would "somewhat" transfer to real-world driving. The remaining 38 percent believed a high degree of transfer-of-training would occur between simulator training and real-world driving. No participants believed that transfer-of-training would not occur.

Table 73. Frequency and Percentage of Participants' Responses to "Does the Information you Learn in the Truck Simulator Carry Over to Real-World Driving?"

\begin{tabular}{|l|c|c|}
\hline Response & Frequency & Percentage \\
\hline High & 6 & $38 \%$ \\
\hline Somewhat & 10 & $63 \%$ \\
\hline Not at all & 0 & $0 \%$ \\
\hline Total & 16 & $100 \%$ \\
\hline
\end{tabular}

During follow-up questioning, participants were asked about the factors that influence transferof-training from a truck simulator to real-world driving. Participants who stated a "somewhat" level in transfer-of-training from truck simulator to real-world driving believed driver attitudes, and their related factors, would be the most important factor influencing transfer-of-training. These participants indicated that transfer-of-training from a truck simulator to a real truck would depend on the driver's attitude towards simulator-based training, whether or not the driver was consciously altering his/her behavior in response to being observed, the quality of the pre-drive briefings, and the time spent in the simulator (both in individual sessions and across the training experience). Participants who reported a high level of transfer-of-training from the truck simulator to a real truck believed specific skills (such as responding to skids) and general skills (safety awareness) would be likely to transfer from a truck simulator to a real truck. Participant responses during the discussion are provided in Table 74. 
Table 74. Participant Responses and Comments Regarding Truck Simulator Transfer of Training

\begin{tabular}{|l|l|}
\hline $\begin{array}{c}\text { Transfer of } \\
\text { Training }\end{array}$ & \multicolumn{1}{c|}{ Participant Comment } \\
\hline Somewhat & Depends on attitude and behaving specifically for trainer. \\
\hline Somewhat & Depends on driver attitude. \\
\hline Somewhat & Depends on the pre-training explanation. \\
\hline Somewhat & Confidence in actions. \\
\hline Somewhat & Depends on repeated exposure through the simulator. \\
\hline Somewhat & Needs more time. \\
\hline High & $\begin{array}{l}\text { Alley and docking transfers well, probably highest transfer-of-training } \\
\text { for entry-level drivers. }\end{array}$ \\
\hline High & Attitude and awareness more than specific behaviors. \\
\hline High & Safety behaviors will transfer. \\
\hline High & Reactions to certain situations. \\
\hline High & Training will transfer. \\
\hline High & Lessons will transfer. \\
\hline High & Overall training will transfer. \\
\hline High & More for hands-on stuff you are not likely to experience. \\
\hline High & Specific training will transfer, such as skids. \\
\hline
\end{tabular}

When specifically asked by the moderator if any negative transfer-of-training could occur between the truck simulator and a real truck, some participants noted that situational awareness and an understanding of traffic flow patterns would be unlikely to transfer from a truck simulator to a real truck (resulting in a reduction in driving performance). One participant who believed situational awareness would not transfer from simulator-based training to real-world driving stated that the simulated drive should always be accompanied by a BTW test of driving ability; this participant stated that although the real world rarely presents complex driving challenges (such as those presented during simulator driving), the overall awareness of driving safety can only be learned and assessed in BTW driving. The participant who reported traffic flow as being unlikely to transfer from a truck simulator to a real truck believed the simulator presented an unrealistic depiction of traffic patterns. More specifically, this participant noted that drivers in the opposite travel lane did not slow to observe a crash in the simulation (as they would in the real world). In this participant's opinion, the simulator did not depict this type of interaction, leading him to believe an entry-level driver would falsely expect similar situations to occur in the real world.

One participant compared simulator-based training to sharpening his driving "tools" by indicating that simulator-based training was about "sharpening the tools ... and building them even more.” Another participant agreed with this statement, stating the simulator-based training was "geared towards being proactive.” This prompted other participants to describe situations where they experienced unexpected events while driving. These participants held the opinion that the specific high-risk scenarios encountered during simulator-based training would lead to greater situational awareness out on the road. 
All participants, regardless of their belief in the realism of the simulated scenarios, reported that the truck simulator would provide drivers with some beneficial transfer-of-training to a real truck. When describing the transfer-of-training from the truck simulator to a real truck, one participant stated, "You are still taking tools into the [real] truck which you didn't have before." Another participant stated, “Although it didn't feel the same, the knowledge is still there.” One veteran driver thoughtfully stated, "If you experience these things on the simulator and you've got the information in your head, it may save your life. Yeah, it doesn't feel the same, guys. You lose a tire at $70 \mathrm{mi} / \mathrm{h}$ and it's a whole different world than what's on the simulator. But the principle is still there, and you're not going to be that shocked when it happens.” This statement led another participant to indicate that emergency maneuvers encountered in the truck simulator increased his confidence in driving, "You know how many videos of [steering tire] blowouts I've watched? But before I did it on the simulator I've always had the fear of the blowout."

Summary: All participants believed transfer of training would occur between the simulator and BTW driving. Driver situational awareness was viewed as likely to transfer. In addition, many skills, such as reactions to events and backing, were viewed as likely to transfer.

\subsubsection{Topic 6: Best Practices and Areas to Improve}

At the conclusion of the focus group meeting, participants were asked by the moderator to describe the principal areas in their truck simulator training program they would retain, as well as modify, if they were in charge of the truck simulator program. The question was structured as a "Brief the President" activity where participants gave a succinct response to questions, as if briefing the president of their carrier on what to retain and modify in their simulator-based training program. Responses were solicited from every participant in the focus groups.

In addition, the moderator asked participants to give an overall rating of their truck simulator program (from 1-10, with 1 being highly ineffective and 10 begin highly effective). Figure 47 presents participant ratings of the effectiveness (or ineffectiveness) of their truck simulator training program. On average, participants ranked their truck simulator training program as "highly effective." The average rating reported by participants was $8.3(S D=1.9)$. The mode of participants’ ratings was 10. 


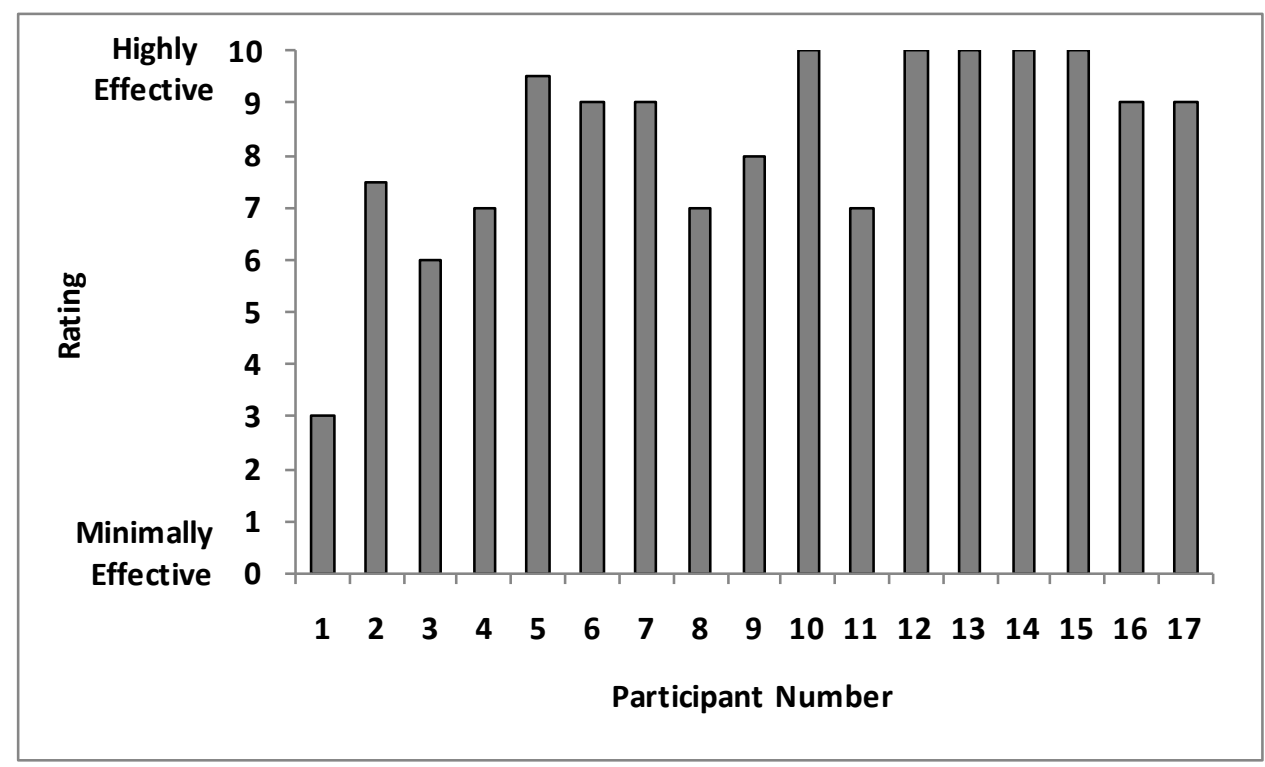

Figure 47. Graph. Participant ratings regarding the effectiveness (or ineffectiveness) of their truck simulator training program.

As participants tended to have very positive views of the simulator-based training program (as evidenced by the high ratings in Figure 47), the principal areas that participants indicated they would like to retain in their truck simulator training program covered broad areas. When participants' responses were grouped as broad categories, six participants reported they would like to retain the specific driving scenarios used in their truck simulator training program, while four participants reported that the proactive nature of the simulator-based training program should be retained and seven participants believed the specific skills training was the area that should be retained in their simulator-based training program. Figure 48 displays the frequency of participant responses to the request, "Describe the principal areas in your truck simulator training program you would retain.” 


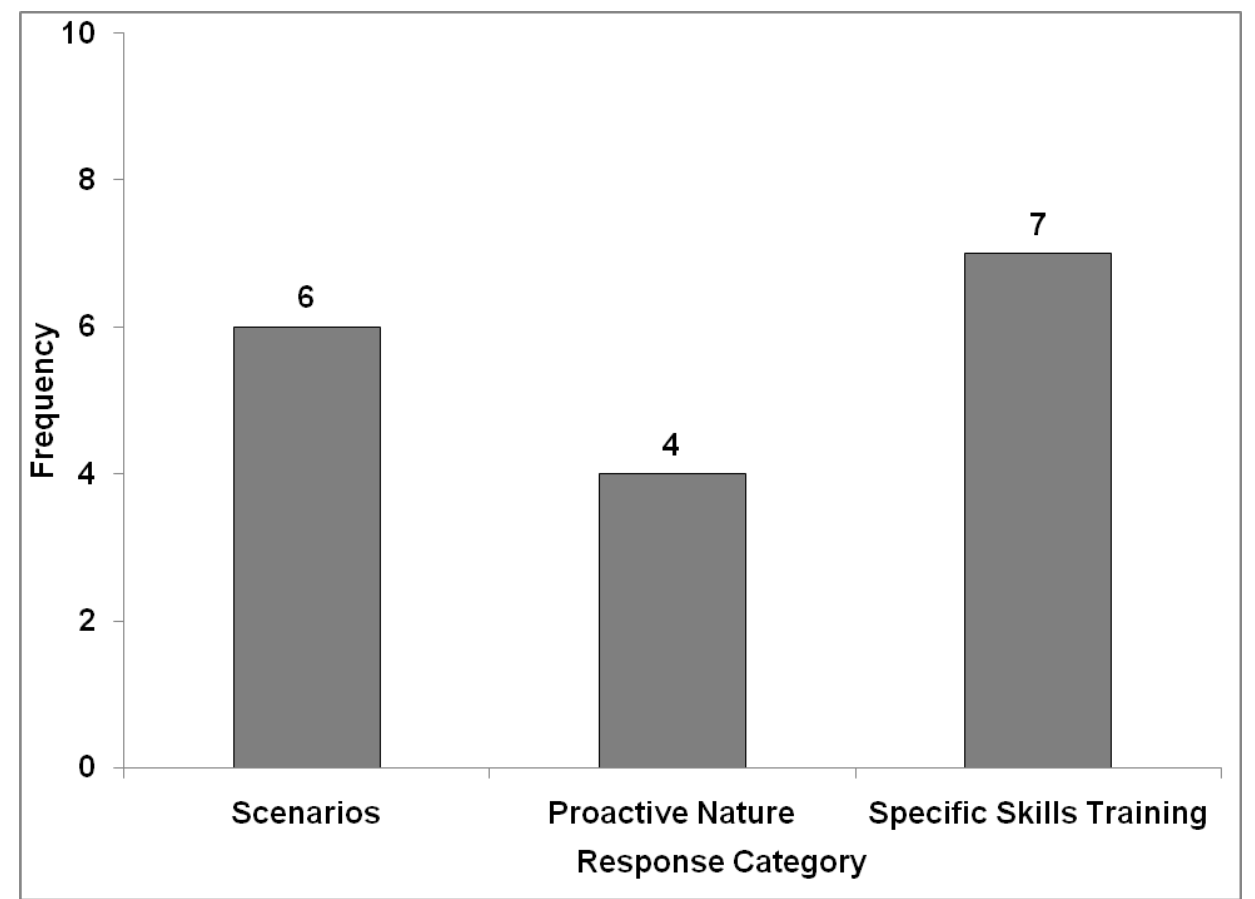

Figure 48. Graph. Participant responses to "Describe the principal areas in your simulator program you would retain"

Participants' responses regarding the principal areas they would like to modify in their truck simulator training program fell into three general categories:

Suggested modifications to the simulator (reported by eight participants).

Additional uses of the simulator (reported by five participants).

Suggestions to change the procedure and implementation of the simulator within the carrier (reported by three participants).

One participant responded that no changes were needed.

As mentioned above, the suggestions for changing the simulator-based training program primarily focused on modifying and adding to the existing training program. Specific suggestions received for changing the program included:

Improving the steering and braking feel of the simulator.

Using a truck cabin as a built-up cabin for the simulator.

Adding a motion base to the simulator.

Improving the visual quality (fidelity) of the simulator.

Specific suggestions for additional uses of the simulator included:

Using the simulator for training additional skills, such as handling misrouting or dealing with fatigue. 
Adding multiple scenarios, some with no critical events, to decrease predictability of each scenario.

Increasing the amount of time spent with each scenario.

Increasing the total amount of time with the truck simulator.

Specific suggestions for changing the procedure and implementation of their carrier's simulatorbased training program included:

Paying drivers for time in training.

Improving the pre-drive briefing to better explain the purpose of training on the simulator.

Making observation less salient by removing the trainer from the room during the drive.

Having both positive and negative feedback at the completion of the drive.

Figure 49 displays the frequency of participant responses to the request, "Describe the principal areas in your truck simulator training program you would modify.”

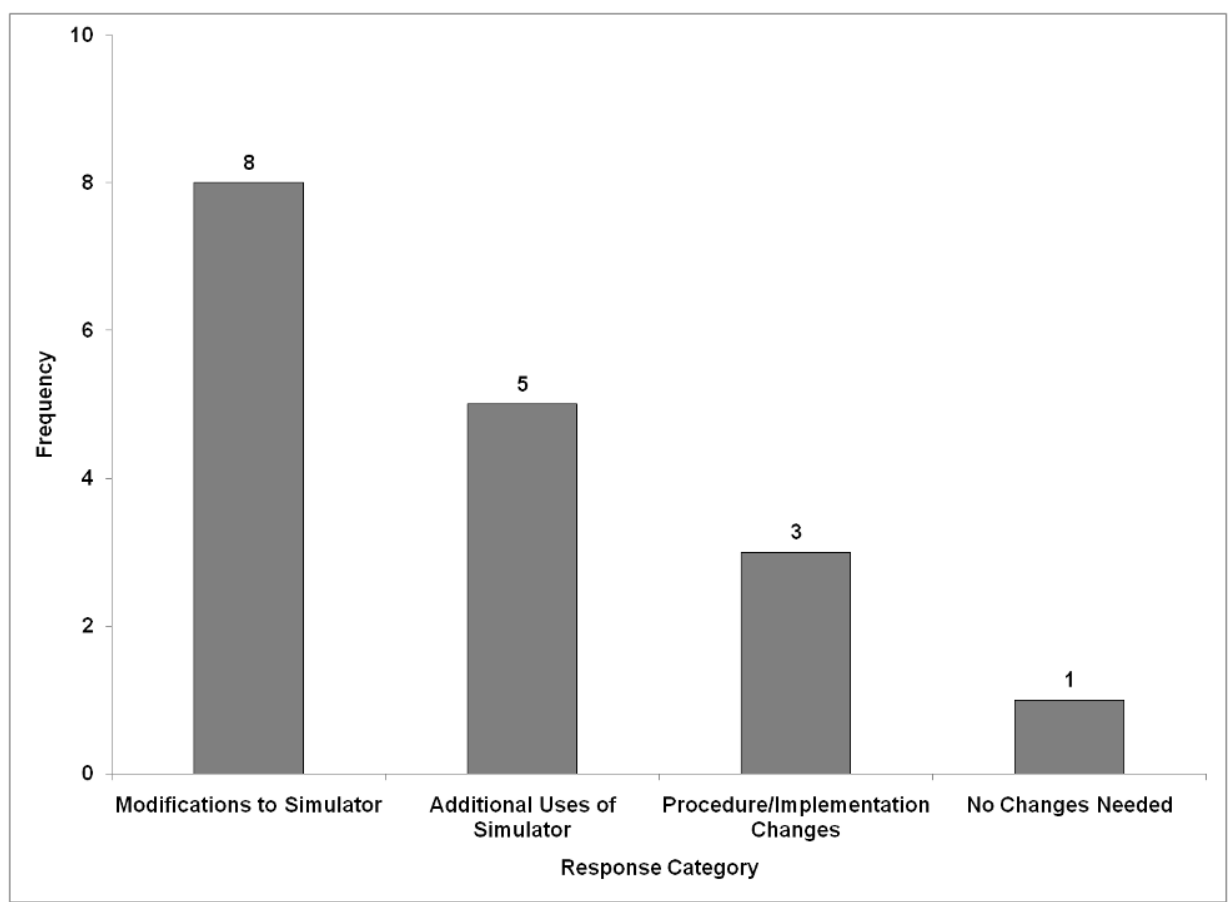

Figure 49. Graph. Participant responses to "Describe the principal areas in your simulator program you would modify"

Summary: Participants appreciated the training for specific skills, realistic scenarios, and proactive safety training provided through the simulator. The participants provided some constructive feedback on areas to improve: modifying the simulator for additional realism, using the simulator in new ways, and changing how the simulator training is implemented. Almost all participants rated their carrier's simulator-based training program as highly effective. 


\subsubsection{Limitations}

Two practical limitations must be kept in mind with respect to these focus group meetings. The participants in both focus groups had recently completed simulator-based driver training (in both cases, the day before). The limited amount of time between participants completing simulatorbased training and participating in the focus group may have biased their responses to the moderator's questions. Although the details of the simulator-training sessions were likely salient to participants, it's likely that participants did not form thorough opinions regarding their simulator training experiences. Additionally, Carrier B focus group participants were all newly employed at the carrier. Although many of these participants arrived at Carrier B with prior driving experience, their experience with Carrier B and its existing training program was limited. This, in turn, may have restricted the scope of these drivers' opinions regarding Carrier's B truck simulator training program.

\subsubsection{Recommendations}

Based on the results from the two focus group meetings, several recommendations for implementing truck simulator training programs in CDL training were apparent, including:

Better pre-training briefings provided by the trainer, which show a clear integration of the simulator-based training with carrier safety management techniques and training policies.

Carriers should portray truck simulator training as a positive, proactive approach to safety.

Carriers should offer drivers the opportunity to have "exploratory training” at their discretion. This can include informal training requests, as well as providing virtual skidpad time.

Ensure that trainers' feedback and coaching takes advantage of the record and playback capabilities of the simulator during training exercises.

Provide a variety of training scenarios that are continuously updated and/or revised to reduce familiarity.

The truck simulator hardware and software should mimic the power units and trailers used by each driver.

Although the truck simulator's visual fidelity is important, important visual aspects of a real truck, such as larger blind spots (requiring head movements) and poor visibility should be included in the simulations.

As participants believed that experiencing simulated scenarios that paralleled their actual driving environment provides the greatest benefit, scenarios should replicate each driver's current or upcoming driving environment (especially in the modeling of terrain and traffic). Scenarios should introduce drivers to challenges within a familiar driving environment.

Participants indicated that the repeatability of the same simulated training scenarios was a powerful method to ensure transfer-of-training.

Participants reported the truck simulator had many unexplored capabilities and was being underutilized in its current format. Researchers and vehicle-simulation companies should 
explore other training uses of the simulator, including fatigue management, misrouting, and driver distraction risks.

\subsection{SUMMARY AND CONCLUSIONS}

\subsubsection{Truck Simulator-Based Training}

Simulator-based training has a relatively brief history. Early development of advanced flight simulation equipment led to the widespread adoption of, and research in, simulator-based training. The success of flight simulation experienced by the U.S. Army Air Force influenced the adoption of simulator-based training approaches in civilian organizations. This was followed by an increase in the research and development of driving simulators.

There are many different configurations of truck driving simulator available. Brock et al., (2001) provided a convenient taxonomy for describing simulators which, viewed through the SRK framework (Rasmussen, 1983), assist in understanding the various types and applications of truck driving simulators. The simplest training simulators, such as PTTs, allow drivers to train on basic skill- and some rule-level tasks. While these simulators are not able to train drivers on the universe of driving tasks, they typically have lower acquisition costs and many allow for multiple students to train simultaneously. Also, these PTTs are typically open-loop, with very low immersion and fidelity. More complex truck simulators provide the ability to train drivers on skill-, rule-, and some knowledge-level tasks. These truck simulators are more complex, relying on computer-generated graphics, and have more valid driver interfaces. Compared to PTT-type simulators, these complex truck simulators have higher acquisition costs and lower student throughput.

Simulator-based training offers several advantages over traditional, BTW training. First, simulators offer increased driver and vehicle safety compared to BTW training. Drivers are exposed to hazardous or challenging situations in a safe and controlled environment with little injury risk. Simulator-based training is also more efficient in terms of time to complete training and trainee throughput. Second, simulator-based training has demonstrated significant cost savings compared to BTW training (simulators have reduced wear and maintenance requirements associated with training). Lastly, simulators offer the ability to obtain high precision measurements of driver performance, which can be shared with trainees to provide feedback and coaching that would be difficult to duplicate in a real truck.

However, simulators can cause some people to experience physical discomfort. This has been termed simulator sickness and typically manifests as nausea, disorientation, and eye strain (Kennedy et al., 1993). However, the potential for these symptoms can be predicted with some success (Golding, 2006).

\subsubsection{Carrier Implementation of Simulator-Based Training}

Two carriers agreed to participate in the current case study. Both had varying approaches to truck simulator-based training. These differences were possibly due to differences in each carrier's approach to driver hiring practices. Whereas Carrier A did not hire drivers without a Class A CDL, Carrier B accepted non-licensed individuals into their entry-level training program (which concluded with the trainee earning a valid license). This was reflected in each carrier's approach 
to truck simulator-based training. Carrier A used truck simulator training primarily for regularly scheduled refresher training and targeted training for specific driver problems, while Carrier B used truck simulator training with both new drivers and during regularly scheduled refresher training.

Carrier A provided the research team with quantitative data to measure the effectiveness of their truck simulator-based training. This data set represented three different training methods (BTW, blended, and simulator) and included data on measures of safety and exposure (i.e., mileage) data from almost 2,500 drivers across a variety of different terminal locations. A survival/failure analysis was conducted on driver tenure (in terms of time of tenure and mileage during tenure) and crash involvement (in terms of time to crash). Driver tenure appeared to be positively affected by the truck simulator-based driver training, with both simulator- and blended-trained drivers having significantly longer duration of tenure and miles logged as compared to BTW trained drivers. Note that it is not possible to state with certainty if simulator or blended training was superior to BTW training with respect to driver tenure as other unexamined explanatory factors not measured were likely to have influenced driver tenure.

The majority of drivers (approximately 94 percent) had zero crashes recorded during the measurement period. When examined by training type, hazard ratios calculated during the survival/failure analysis revealed that drivers in the simulator- and blended-trained groups had a higher likelihood of a crash earlier in their tenure as compared to BTW drivers. However, the pragmatic effect of this difference was negligible due to the small difference between groups (i.e., 2 days). The cost per crash was analyzed with descriptive statistics due to a severe imbalance in the number of observations across each different training type. Drivers that received simulator training had the lowest overall cost per crash and cost per crash per thousand miles traveled compared to both the blended and BTW drivers.

\subsubsection{Driver Opinions on Simulator-Based Training}

Drivers from both carriers who recently completed truck simulator-based training were asked to participate in a focus group meeting. Almost all participants expressed positive opinions regarding their own experiences in truck simulation-based training, believing that all drivers would obtain some benefits from training in the truck simulator. Some similarities, such as the physical reactions in the truck simulator, and differences, such as the increased safety of the simulator-based training, were identified by participants in regards to comparisons of truck simulators and real trucks. Most participants believed the truck simulator would be most beneficial in training entry-level drivers and that some degree of transfer-of-training to a real truck would occur. Participants reported that truck simulator training allowed them to experience hazardous, challenging, and infrequently occurring situations which they would not otherwise have the opportunity to train for. Additionally, many participants believed that truck simulatorbased training assisted drivers in increasing their situational awareness in the driving environment.

Participants also noted several issues in truck simulator training. Many participants indicated that the truck simulator was similar to an arcade game. Also, many participants identified "poor driver mindset” (the driver's attitude towards training, and specifically simulator-based training) as a major barrier to the success of a truck simulator-based driver training program. Participants noted that several of these limitations in truck simulators could be addressed with higher fidelity 
simulations and pre-training briefings (which ensure truck simulation-based training is portrayed as positive and proactive).

The majority of participants had positive opinions regarding their carrier's truck simulator training program. In fact, participants rated their truck simulator-based training program as highly effective. All participants reported the truck simulator-based program was a manifestation of each carrier's safety-conscious culture. These participants identified several best practices in truck simulator-based training, including use of scenarios that reflect real-world driving, proactive nature of the truck simulator-based safety training, and training in specific driving skills and maneuvers. Areas for improvement focused on hardware and software modifications to the truck simulator, additional training uses, and modifying how the truck simulator was implemented at the carrier.

\subsubsection{Future Directions}

Truck simulation technology continues to expand at a rapid pace, enabling many new lines of research into CMV driver behavior and training. Truck simulation has demonstrated considerable benefits in the training of entry-level CMV drivers and in the skills-maintenance for experienced CMV drivers. As simulation technology in CMV operations matures and becomes more prevalent in training programs, some key areas that deserve further exploration are noted below. Further exploration of these issues will aid in the proper implementation of truck simulator-based training programs.

Is there a need for standardized entry-level driver training during truck simulator driving scenarios?

What are the "best practices" to ensure transfer-of-training between a truck simulator and a real truck?

What is the optimal amount of training time during truck simulator-based refresher training to ensure transfer-of-training to a real truck?

What is the optimal ratio of training time in a truck simulator and a real truck during entrylevel driver training? 
[This page intentionally left blank.] 


\section{DISCUSSION}

Simulator-based training has a relatively brief history. Early development of advanced flight simulation equipment led to the widespread adoption of, and research in, simulator-based training. The success of flight simulation experienced by the U.S. Army Air Force influenced the adoption of simulator-based training approaches in civilian organizations. This was followed by an increase in the research and development of driving simulators.

There are many different configurations for truck driving simulators available. Brock et al., (2001) provides a convenient taxonomy for describing simulators that, viewed through the SRK framework (Rasmussen, 1983), assists in understanding the various types and applications of truck driving simulators. The simplest training simulators, such as PTTs, allow drivers to train on basic skill- and some rule-level tasks. While these simulators are not able to train drivers on the full spectrum of driving tasks, they typically have much lower acquisition costs and many allow for multiple students to train simultaneously. Also, these PTTs are typically open-loop, with very low immersion and fidelity. More complex truck simulators provide the ability to train drivers on skill-, rule-, and some knowledge-level tasks. These truck simulators are more complex, relying on computer-generated graphics, and have more complete driver interfaces. Compared to PTTtype simulators, these complex truck simulators have higher acquisition costs and lower student throughput.

Simulator-based training offers several advantages over traditional, BTW training. First, simulators offer increased driver and vehicle safety compared to BTW training. Drivers are exposed to hazardous or challenging situations in a safe and controlled environment with little injury risk. Second, simulator-based training is also potentially more efficient in terms of time to complete training and trainee throughput. Third, simulator-based training has demonstrated significant cost savings compared to BTW training (simulators have reduced wear and maintenance requirements associated with training). Lastly, simulators offer the ability to obtain high precision measurements of driver performance, that can be shared with trainees to provide feedback and coaching that would be difficult to duplicate in a real truck.

However, some forms of driving simulators presenting computerized graphics, and especially simulators with wrap-around displays, can cause some people to experience physical discomfort. This has been termed simulator sickness and typically manifests as nausea, disorientation, and eye strain (Kennedy et al., 1993). However, the potential for these symptoms can be predicted with some success (Golding, 2006).

The results from the SimVal2 project presented herein involve several areas of interest: (1) entrylevel training study, (2) advanced capabilities showcase, (3) cost benefit analysis of entry-level CMV driver training, and (4) a case study on existing simulator-based training programs. The following is a summary of those findings and some of the main aspects of these four areas. 


\subsection{ENTRY-LEVEL TRAINING STUDY}

\subsubsection{Effects of Training Method on CDL and Related Test Performance}

Two main areas were evaluated:

Simulator students’ capability to perform as well as the other training methods.

Non-certified methods performance compared the certified ones.

First, the results suggest that DTCC participants who received part of their training in a truck simulator do not significantly differ in test performance as compared to DTCC participants who received all of their training BTW. Second, when looking at the different training methods there are statistical differences in the DMV range test results (no difference in the DMV road test), however these differences are so small as to not be practical differences. On average, all training methods allowed participants to pass the DMV tests with an average score above 90.

However, in addition to DMV tests, performance results on the DTCC and Simulator road and range tests were compared. This is an important comparison as the DTCC and Simulator tests were constructed as a replication of DMV tests. Additionally, the scoring on these tests was validated by an independent reviewer. Group differences were present in both DTCC and simulated versions of the road and range tests. For the DTCC and Simulator road tests, this difference was manifest as the Conventional and Simulator groups (both having received PTDIcertified training) scored significantly greater than the Informal and CDL-focused groups (both not having received certified training). Similar results were present for the DTCC range test, with the Conventional and Simulator groups scoring significantly greater than the Informal or CDLfocused groups. On the Simulator range test, the Simulator training group scored significantly greater than the three other groups. These statistical differences suggest that the DTCC students, on average, score better than the drivers that are not trained in a PTDI-certified training program.

\subsubsection{Validity of Simulation Training and Testing}

\subsubsection{Assessing Road and Range Driving Skills}

One of the main reasons for the interest in using simulators for testing is to alleviate the costs and requirements that a BTW test might imposed. Therefore, it is important that the results obtained in a simulator are highly correlated to the ones obtained when the person is tested in a real vehicle either at a training school or at the DMV in order to obtain the full benefits of an alternate test modality.

Based on the results obtained in this research effort, only moderate to low correlations between simulator and BTW tests were observed across most groups. The only training group with a significant correlation between the Simulator tests and DMV test counterparts was the simulator training group. However, even within the simulator training group, the correlation for the range test was not a high one.

DMV scores were not correlated to the Simulator test scores for the groups that were not trained in a simulator. Moreover, all 9 informal training group participants failed the Simulator road test and at least one participant from each training group failed the Simulator road and range test 
(including the Simulator Group). This was not the case for the DTCC and DMV tests. Multiple participants from all groups were able to pass both sets of tests. Only participants from the Conventional and CDL-focused Training Groups had a significant, albeit moderate, correlation between the Simulator and DTCC road tests.

These results must be considered in future decisions that involve simulator-based driver testing. Unsurprisingly, participants trained in a simulator are able to obtain higher scores in simulatorbased testing than the ones not trained in a simulated environment. Moreover, participants that are not trained in a simulator might not be able to successfully pass a simulator CDL test, even if they would have passed a BTW version of the same test. There are several aspects that need to be experienced and practiced in a simulator in order to be mastered in a simulated test environment. One of the potential aspects that might have hindered non-simulator trained drivers is depth perception. Depending on the simulated environment or the task being tested, several landmarks and queues obtained through depth perception are not present in a simulated environment. This resulted in tasks such as backing being more difficult in the simulated environment.

\subsubsection{Simulator versus Behind-the-Wheel Testing}

Thirteen road test and 6 range test scoring areas were compared between Simulator and DTCC tests. Not all 13 road test scoring areas had a significant correlation between the Simulator and DTCC tests. However, scoring aspects related to general driving and turns had significant correlations for the Conventional and Simulator Groups. None of the road test scoring aspects for the Informal and CDL-focused Group participants were correlated between the Simulator and DTCC tests. Further analysis comparing each participant's Simulator to DTCC test on the 13 scored aspects revealed a number of differences. In Simulator testing, the Conventional Group had had more errors in general driving and right turns, and fewer road sign errors as compared to DTCC tests. The Simulator Group had a lower number of left curve and left turn errors, and a greater number of right turn, left lane change, and right lane change errors in the Simulator version of the road test.

Further analysis of LD rates indicated that there was a difference in the rate of LD in DTCC and Simulator road tests by group. Participants in the conventional training group had a greater rate of LD in the Simulator road test as compared to the DTCC road test. No other group displayed any reliable differences in LD rates based on test mode. As with the range tests, the lack of rich perceptual depth cues in the simulated environment may be one reason for this. Note that both Informal (all participants failed the Simulator road test) and CDL-focused (only one participant passed the Simulator road test) Training Groups had low performance on the Simulator road test. Thus, this pattern may not have manifested as a greater rate of LD due to the tests stopping earlier for almost all the participants in these groups.

Additionally, the Simulator and Conventional Group were shown to have similar LD rates, indicating that the participants who received most of their training in the simulator had vehicle lateral control skills equivalent to those of conventionally trained participants. This suggests that the simulator has validity for training entry-level CMV drivers for road driving. Similarly, when those participants who experienced PTDI certified training (the Conventional and Simulator Groups) were compared against the participants who did not have PTDI certified training, a 
difference in LD rate was observed. In the DTCC road test, drivers with PTDI certified training had a significantly lower LD rate than those drivers who did not have PTDI certified training.

This suggests that for the road test the results obtained in a simulator will not always resemble the results obtained BTW. While some participants may be able to demonstrate their skills in a similar manner in both environments (i.e., simulated and BTW), overall the results obtained in one environment may not be related, at least not a linear fashion, to results obtained in the other. Based on the present results, drivers trained in a certified program were the only ones able to demonstrate their general driving skills in both a Simulator and BTW (e.g., shifting, clutch use, gear selection, braking, steering, lane usage, and traffic checks). It is important to mention that it was confirmed by an external subject matter expert in the field that no bias in the skill test scoring system existed for any of the four training groups when they were evaluated in the simulated and BTW environments at DTCC.

The results from the range test are not parallel to the ones obtained in the road test. For the range test the Simulator Group was the one that obtained significant correlated results between the two test environments for most of the scoring aspects. The CDL-focused group was able to demonstrate their skills in a similar manner in both environments for the alley dock. However, this result is based on four comparisons. Straight line backing was the only scenario that did not present similar results in both testing environments for any of the groups. The main reason for this might be that depth perception cues are needed for such maneuver and they are not present or fully manifested in the simulated environment. Further analysis comparing each participant's Simulator to DTCC test on the 13 scored aspects revealed a number of differences. In Simulator testing, the Conventional Group had had more errors in general driving and right turns, and fewer road sign errors as compared to DTCC tests. The Simulator Group had a lower number of left curve and left turn errors, and a greater number of right turn, left lane change, and right lane change errors in the Simulator version of the road test. Again, these findings show that the tests are not equivalent for participants trained primarily in the simulator or for participants who trained in a conventional, BTW, manner. However, the lack of a significant difference in final range test scores between Conventional and Simulator Groups suggest that the simulator is a valid method of training entry-level CMV drivers.

\subsubsection{Simulator Most and Least Effective Features}

The results obtained in this subjective assessment suggest that basic controls, repeatability, and overhead views are the top three most effective simulator aspects. Similar to the findings of the correlational analysis, the simulator allows drivers trained in this driving environment to practice the basic controls needed for a vehicle (refer to it in the road test as general driving skills). This accords with previous research findings (Robin et al., 2005b) suggesting that repeatability and the ability to obtain overhead views and other perspective of where the vehicle is positioned is a very important aspect of a simulator-based training program. These findings confirm that having the ability to practice basic controls in a repeatable manner and having features such as overhead views during training, are just some of the main aspects why a simulator should be considered for training purposes.

The top ranked least effective features based on the assessment were realism and backing. While the realism of simulators has improved dramatically over the years, a simulated environment is still not capable of portraying or potentially engaging a driver the same way a real-world 
scenario might be able to. However, not all scenarios could be repeated safely and economically in a real-world environment. Backing (part of the range test scenarios) was highlighted by many of the analyses as a problematic area for simulator training. The depth perception cues needed to successfully accomplish this task are simply not presently available in an environment with only two dimensions (2D).

\subsubsection{Participant Evaluation of Training after Employment}

Due to the economic downturn in the last few years, many participants were not able to find a job as a Class A CDL licensed CMV driver. Therefore, the results presented herein are restricted to a small sample. However, the trend presented in these findings seems to be consistent across all four training groups. All drivers thought they were better than average in terms of control and safety skills. On average, all drivers thought their training method was average or above average. Some drivers found potential deficiencies in their program after they were driving professionally; however, on average, most ratings were no different from their original rating of their training method or better. One exception to this was found in the conventional training group. The Conventional Group always rated their training method equal or better than they originally rated it at the conclusion of entry-level training. When they were evaluated in a simulated environment, drivers thought that the simulator assessment was fair. Most of the drivers that participated in the study, and were currently employed as professional drivers, would recommend the training method they selected to obtain their entry-level training. Only one participant would not recommend the training method he selected (i.e., Informal).

\subsubsection{Effects of Training Method on Driver Performance}

The results obtained in the longitudinal follow-up were surprising. Most of the follow-up test scores were lower than the original scores for the entry-level tests. However, Conventional and Simulator Groups performed better on the follow-up road tests than the Informal and CDLfocused ones. Other possible explanatory factors that may have had an effect on follow-up road and range test performance includes the transmission type of the participant's work vehicle, the type of backing maneuvers encountered on the job, differences in driving exposure (i.e., local delivery versus over the road), and the lack of behavioral reinforcements while on-the-job. A number of participants mentioned that the CMVs they drove on the job were equipped with autoshift or synchronized transmissions. Thus, these participants would not have continued practice and exposure to the task of shifting a double-clutching, non-synchronized, manual transmission and its associated sub-tasks (e.g., engine speed matching, gear selection, double clutching, use of range switches). Additionally, some participants may not encounter all of the CDL test backing maneuvers (i.e. parallel parking) at their place of employment. Thus not having continued exposure to these skills would lead to deterioration in skill proficiency. Likewise, the lack of behavioral reinforcement for unsafe behaviors could have lead to some participants' demerits during testing.

\subsubsection{Effects of Training Method on Safety Performance Based on Driving Records}

The fact that the CDLIS records did not show the crashes is an important finding by itself. This type of database is viewed as the gold standard for many research efforts. However, finding out that multiple crashes are never reported and that driving records would never show the true reason why a driver was terminated is very important. Based on the present results, all groups seem to have a similar number of violations per participant and no major differences were found 
based on safety records. However, conclusive results are not able to be drawn based on the small sample.

\subsubsection{Effects of Training Method on Employee’s Supervisor Evaluation}

No major differences existed in the ratings for the participants that were evaluated by their supervisors. All training groups received a control and safety skills rating above average with a tendency towards the side of best performance. Obtaining employee evaluations, similarly to a reference, is becoming more difficult due to corporations viewing employee evaluations or references as a potential source of litigation. Although supervisors were reassured that the evaluation was for research purposes only and that their identity would be confidential, only 29 percent of the supervisors were willing to collaborate. Additionally, some participants who are owner-operators, as well as one employer who is no longer in business, were removed from the results.

\subsubsection{Exploratory Analysis of “Big Five” Personality Correlates}

No differences in any personality factor based on group membership were present, indicating that the groups had very similar personality traits. As there were no group differences in any of the 5 factors, regression analysis was conducted to examine for personality factor influences on test outcomes. No significant effects of personality were present for any of the tests. In all cases training group membership was the only significant factor in the model. In addition, the group membership effect provides a similar result as the examination of DMV, DTCC, and Simulator road and range scores (see previous topics). Therefore, it is suggested that skill test scores are predicted by training group membership and not personality factors.

\subsection{ADVANCED CAPABILITIES SHOWCASE}

\subsubsection{Assessing Advanced Capabilities of the Simulator}

No major statistical differences exist in the expected driving performance of million-mile participants and non-million milers for the showcase scenarios. When overall performance during emergency maneuvers and extreme conditions is evaluated, an expected trend is observed of the majority of the participants responding to the scenario appropriately. Overall, million milers responded appropriately more often than non-million milers.

\subsubsection{Extreme Conditions}

While driving the simulator, participants encountered 10 different extreme conditions. The extreme driving conditions were designed to replicate many of the conditions they may encounter on a daily basis while driving a tractor trailer in the real world. While both million milers and non-million milers tended to respond appropriate in these scenarios, the million milers tended to respond appropriately more often than the non-million milers. Million milers across all trailer configurations responded appropriately approximately 68 percent of the time, while the non-million milers responded appropriately approximately 62 percent of the time. However, when observing the extreme conditions on an individual basis, a rather limited number of these conditions produced the majority of the inappropriate responses for both the million milers and non-million milers. Details about each of the conditions are detailed in the results chapter. 
Of the million-mile participants, the tanker participants had the highest rate of appropriate responses at 73 percent, while those million-mile participants who pull a van trailer had the lowest rate of appropriate responses at 63 percent. This trend is of interest as the dynamics of each trailer type is substantially different. Tanker trailer dynamics typically present the most challenging conditions for a participant, followed by the doubles trailers, then the van trailer. It would be expected that million-mile tanker participants should have a higher degree of vehicle awareness than those participants who have only pulled van trailers.

\subsubsection{Emergency Maneuvers}

While driving the simulator, participants encountered 12 different situations requiring emergency maneuvers. These situations were designed to assess the participant's ability to perform emergency maneuvers when needed while driving. While appropriate responses were observed across both million milers and non-million milers in the majority of trails, million milers tended to respond appropriately more often than the non-million milers. Million milers across all trailer configurations responded appropriately approximately 70 percent of the time while the nonmillion milers responded appropriately approximately 62 percent of the time. When examining these emergency maneuvers individually, five maneuvers standout. Details about these maneuvers are provided in the results chapter.

Of the million-mile participants, those who pulled a van trailer had the highest rate of appropriate responses at 80 percent, while those million-mile tanker trailer participants had the lowest rate of appropriate responses at approximately 65 percent. This follows an expected trend. Tanker trailer dynamics typically present the most challenging conditions for a driver, followed by the doubles trailers and the van trailer, respectively. It would be expected that million-mile participants who pull a van trailer should have a higher degree of vehicle handling and control skills than those participants who pull tanker trailers, as van trailers are the most stable of the three trailers used for this showcase.

The results of this showcase demonstrate that while million-mile participants respond appropriately more often than non-million-mile participants in both the emergency maneuvers and extreme conditions. However, the million-mile participants still responded inappropriately or not at all for approximately 30 percent of the emergency events and approximately 32 percent of the extreme conditions encountered. This is an indication that all participants, including million milers, could potentially benefit from refresher and defensive driving type training. The simulator provides one such mechanism for providing this type of training. As noted in the Case Study of Existing Driver Training Programs (Chapter 7), some larger carriers are beginning to use driving simulators for this purpose. Thus, the simulated scenarios described here could be used as part of a training program for such refresher and defensive driving training.

\subsubsection{Participants’ Assessment of the Simulator’s Advanced Capabilities}

Following the completion of the scenarios, both million-mile and non-million-mile participants for all three trailer configurations provided feedback on both the usefulness and realism of the simulator, as well as feedback on which emergency maneuvers and extreme events were most realistic. These ACS participants completed 12 emergency maneuvers and 10 extreme condition scenarios. No overall pattern of statistically significant differences between million milers and non-million milers exist in the ratings of the showcase scenarios' realism. When overall 
participant ratings for the emergency maneuvers and extreme conditions are evaluated, a trend is observed of the majority of participants feeling these showcase scenarios are realistic when compared to their real world counterpart. However, the black ice extreme condition proved difficult to replicate in the simulated environment. Participants noted that several realistic driver cues in black ice conditions were missing from this scenario.

When participants' feedback on both the usefulness and realism of the simulator is evaluated another trend is observed. Both million milers and non-million milers for the doubles trailers and tanker trailer rated safety and safe operations in the top three most effective aspects of the simulator. This is an important finding, as these participants agree that both the safety and safe operations ability of a commercial vehicle driving simulator can provide beneficial training and experience for these emergency maneuvers and extreme conditions. Another important aspect for the simulator is vehicle dynamics, especially in relation to the vehicle dynamics of tanker trailers and doubles trailers. Here, simulation provides a safe alternative for exposing drivers to emergency maneuvers, in comparison to the risk of a safety critical event associated with replicating these emergency maneuvers in a real truck-trailer combination. Both million-mile and non-million-mile van trailer participants rated either safety (million-mile van trailer participants) or safe operations (non-million-mile van trailer participants) as one of the top three aspects. However, neither rated both safety and safe operations together in the top three. This trend may be explained by the fact van trailers do not present the same vehicle dynamics challenges of the other trailer configurations. Thus, participants who pull van trailers may feel that safety and safe operations provided by the simulator is not as critical of a factor when training and practicing on a simulator.

Participants were also asked to provide open ended comments. Some of the most notable comments were to add night driving, high bridges with wind gusts, include more city driving, and provide a better replication of the sound of an engine. These were all cues that participants noted as providing feedback, or special situations presenting increased risk of a safety critical event, that they felt would be beneficial for training.

Both doubles trailer million milers and non-million milers had similar feedback on the most useful aspects of the simulator. Doubles million-mile participants rated safety as the top aspect, followed by safe operations (the ability to teach drivers defensive driving skills). Doubles nonmillion-mile participants rated realism as the most effective, followed by safety. Tanker million milers rated realism as the most effective aspect, followed by safety. Tanker non-million milers ranked safe operations as most effective, followed by safety. Van trailer million-mile participants rated safety as most effective, followed by a tie between realism and basic control. Van trailer non-million milers rated enrichment (the ability to simulate rare or otherwise infrequently encountered events such as weather conditions) as most effective, followed by realism and safe operations. Most ACS participants viewed the safety aspects, including both the direct safety of training in a simulator and the indirect safety of using the simulator to train defensive driving, as the most beneficial aspect of driving simulation.

The ACS participants also provided ratings of each of the emergency maneuvers and extreme conditions. Both million milers and non-million milers, in each trailer type, rated the scenarios as "about the same" or "realistic" as compared to driving a real truck. The most realistic ratings, across all groups, were typically assigned to weather events (fog and snow) and construction 
zones. Comparisons between million-mile and non-million-mile drivers, in each trailer type, demonstrated few differences in subjective ratings. Doubles trailer s rated right side rollovers as more realistic than doubles non-million milers, and tanker trailer non-million milers rated evasive maneuvers as more realistic than tanker trailer million milers.

These findings indicate that the simulator is able to produce a realistic simulation of many different types of driving environments. Most drivers held favorite ratings for the ability of the simulator to replicate potentially hazardous situations in a safe manner, and to be used to train drivers in defensive driving skills. Similarly, the emergency maneuver and extreme condition scenarios were deemed to be generally realistic by participants with very few differences between million milers and non-million milers in ratings. This suggests that the realism of the events encountered on the simulator are not just limited to a certain group of drivers based on the level or amount of driving experience. However, this study only examined one simulator. The results from this course of research may not generalize to training programs using different simulators. Therefore, an examination of the equipment levels required to provide adequate training in a simulator-based program is justified.

\subsection{COST EFFECTIVENESS ANAL YSIS OF ENTRY-LEVEL CMV DRIVER TRAINING}

The present study attempted to quantify the costs associated with multiple methods of training. Each method of training incurred costs in the development, delivery, and evaluation of the training program.

\subsubsection{Cost of Simulator versus Conventional Training}

Only a small difference in direct costs between the Simulator and Conventional Group training was found. In addition, it is expected that with the advancement of technologies and extended usage, simulator costs will decrease over time while the number of students that can be trained at the same time will increase without any deterioration in the quality of the training. If the expected crashes, based on the number of violations, are incorporated in the analysis, the NPV of the Simulator training method (as compared with the Conventional Training method) is negative. This will render the Conventional Training method as superior. However, when these participants performed the DTCC range and road tests, no significant differences were experienced in their abilities among the different groups. How these abilities translate to safety benefits is uncertain. A follow-up of the drivers to allow for additional data for this analysis at a later point would be beneficial. Potentially, performing a follow-up of a subgroup of participants in each group in a naturalistic manner would render more data (i.e., safety-critical events) than can be obtained from police-reported collisions.

\subsubsection{Cost of Conventional versus CDL-focused and Informal Training}

None of the drivers in the three groups were involved in a crash or traffic violation. Therefore, the BCA for these types of training was reduced to a comparison of the direct costs of training. The Informal Group was the cheapest of the three methods. However, there were large variations in the training time reported by the drivers in this group. On the other hand, the difference in costs was relatively minor (approximately 2.6 percent). Trainee time was a major component for 
the cost of Conventional Group and CDL-focused Group training. For every hour of training in those groups, there is an increase of $\$ 18.61$. The average number of hours per trainee was 190 hours and 300 hours for the CDL-focused and Conventional Groups, respectively. This difference represents a minimum of \$2,047 (110 hours at \$18.61 per hour) in the total cost. It is expected that this increase in the number of hours will result in some type of safety benefit that has not been reflected in the crash and violation data for the period that the data were collected. Some group differences were found between participant performance on DTCC road and range tests. Similar to the above comparison, the ability to translate these differences into safety benefits is uncertain.

\subsection{CASE STUDY ON EXISTING SIMULATOR-BASED TRAINING PROGRAMS}

\subsubsection{Carrier Implementation of Simulator-Based Training}

Two carriers agreed to participate in the case study. Both had varying approaches to truck simulator-based training; these differences were possibly due to differences in each carrier's approach to driver hiring practices. Whereas Carrier A did not hire drivers without a Class A CDL, Carrier B accepted non-licensed individuals into their entry-level training program (that concluded with the trainee earning a valid license). This was reflected in each carrier's approach to truck simulator-based training. Carrier A used truck simulator training primarily for regularly scheduled refresher training and targeted training for specific driver problems, while Carrier B used truck simulator training with both new drivers and during regularly scheduled refresher training.

Carrier A provided the research team with quantitative data to measure the effectiveness of their truck simulator-based training. This data set represented three different training methods (BTW training, a blended simulator and BTW training program, and simulator training program) and included data on measures of safety and exposure (i.e., mileage) data from almost 2,500 drivers across a variety of different terminal locations. A survival/failure analysis was conducted on driver tenure (in terms of time of tenure and mileage during tenure) and crash involvement (in terms of time to crash). Driver tenure appeared to be positively affected by the truck simulatorbased driver training, with both simulator- and blended-trained drivers having significantly longer duration of tenure and miles logged as compared to BTW trained drivers. However, it cannot be stated with certainty if simulator or blended training was superior to BTW training with respect to driver tenure as other unexamined explanatory factors not measured were likely to have influenced driver tenure.

The majority of drivers (approximately 94 percent) had zero crashes recorded during the measurement period. When examined by training type, hazard ratios calculated during the survival/failure analysis revealed that drivers in the simulator- and blended-trained groups had a higher likelihood of a crash earlier in their tenure as compared to BTW drivers. However, the pragmatic effect of this difference was negligible due to the small difference between groups (i.e., 2 days). The cost per crash was analyzed with descriptive statistics due to a severe imbalance in the number of observations across each different training type. Drivers that received simulator training had the lowest overall cost per crash and cost per crash per thousand miles traveled compared to both the blended and BTW drivers. 


\subsubsection{Driver Opinions on Simulator-Based Training}

Drivers from both carriers who recently completed truck simulator-based training were asked to participate in a focus group meeting. Almost all participants expressed positive opinions regarding their own experiences in truck simulation-based training, believing that all drivers would obtain some benefits from training in the truck simulator. Some similarities, such as the physical reactions in the truck simulator, and differences, such as the increased safety of the simulator-based training, were identified by participants in regards to comparisons of truck simulators and real trucks. Most participants believed the truck simulator would be most beneficial in training entry-level drivers and that some degree of transfer-of-training to a real truck would occur. Participants reported that truck simulator training allowed them to experience hazardous, challenging, and infrequently occurring situations which they would not otherwise have the opportunity to train for. Additionally, many participants believed that truck simulatorbased training assisted drivers in increasing their situational awareness in the driving environment.

Participants also noted several issues in truck simulator training. Many participants indicated that the truck simulator was similar to an arcade game. Also, many participants identified "poor driver mindset” (the driver's attitude towards training, and specifically simulator-based training) as a major barrier to the success of a truck simulator-based driver training program. Participants noted that several of these limitations in truck simulators could be addressed with higher fidelity simulations and pre-training briefings (which ensure truck simulation-based training is portrayed as positive and proactive).

The majority of participants had positive opinions regarding their carrier's truck simulator training program. In fact, participants rated their truck simulator-based training program as highly effective. All participants reported the truck simulator-based program was a manifestation of each carrier's safety-conscious culture. These participants identified several best practices in truck simulator-based training, including use of scenarios that reflect real-world driving, proactive nature of the truck simulator-based safety training, and training in specific driving skills and maneuvers. Areas for improvement focused on hardware and software modifications to the truck simulator, additional training uses, and modifying how the truck simulator was implemented at the carrier.

\subsection{LESSONS LEARNED}

During the course of this multi-year research effort, several lessons were learned regarding aspects such as data collection and participant throughput. These lessons learned are summarized here for the benefit of future research activities.

\subsubsection{Participant Payments for Travel Time}

It became apparent during the course of the study the need to pay participants for their travel time. Entry-level participants were recruited from all four DMV locations in the State of Delaware. While the testing location was located near one of these locations (Georgetown), the other three DMV sites were all over one hour's drive away from the testing location. The Dover DMV was one hour from the test site, while the Wilmington and New Castle DMV locations 
were 1.75 hours from the test site. This is important, as participants generally travelled from their home address. Several participants had a one-way travel time of 3 hours. Once the new recruitment plan, including reimbursement for time traveling to the test site, was implemented the number of participants increased.

\subsubsection{Participant Contact}

Contact with participants for job status checks sometimes proved difficult. This difficult was primarily with the CDL-focused Group. A few participants in the other groups were also difficult to contact. A protocol was followed for attempted contacts with the participants: participants were contacted by phone and a voice mail message was left, when participants failed to return voice messages a letter was mailed to their place of residence reminding them of the study and asking them to please contact the researcher at their earliest convenience. If contact was still not established, a second letter was mailed approximately one month later. In some cases either the participant's home or mobile phone numbers were disconnected; on some occasions both telephone numbers had been disconnected. Additionally, some participants moved during the course of their participation.

The Conventional and Simulator Group participants were with the researcher approximately 5 days per week for 8 weeks. The participants were able to become familiar and comfortable with the presence of the researcher and the study protocols, thus being more likely to respond for the follow-up. However, the Informal and CDL-focused Group participants were only with the researcher for one day. This exposure to the researcher over an extended length of time may provide one explanation as to why it was easier to maintain contact with the Conventional and Simulator Group participants.

Payment did not seem to influence participants' likelihood to remain in contact for the follow-up study. Participants were paid \$32 per hour for their time during the follow-up testing, with a $\$ 200$ bonus once they completed all aspects of the follow-up. However, this did not appear to influence participants' choice on continued participation.

\subsubsection{Employer Evaluations and Data}

Only 29 percent of employers collaborated with the research team to complete the participant evaluations. Most employers who refused to complete the evaluations cited liability as a main concern, even though they were assured the data was for research purposes and would remain confidential. Participants also signed an authorization granting the release of this information but this did not help easing the employers’ concerns over liability.

While the researchers were unable to predict who the future employers were going to be, perhaps obtaining these data in future longitudinal studies could be made easier through the use of certificates of confidentiality. These would provide a recognized protection to the employer release of data and may help increase the number of employers willing to provide such data.

\subsubsection{Department of Motor Vehicles Data}

Test scoring data handling by DMVs was found to vary from State to State. While scoring data (i.e., CDL range and road tests) were able to be obtained from the Delaware CMV, it was found 
that the Maryland DMV did not keep records of road and range test scores. Once this was determined, participants were no longer recruited from Maryland.

Additionally, at the beginning of the study, there was an approximately 3 week delay from the time a person took the CDL range and road tests until their scores were accessible on the Delaware DMV database. However, during the last months of the study, the Delaware DMV reduced this delay to only one day. Also, following the implementation of this new procedure, the entire range and road tests score sheets began being made available through the database.

The quality control of DMV records is one aspect the researchers are unable to control. This should be taken into consideration in future studies like this.

\subsubsection{Simulator Sickness}

Simulator sickness was a problem with ACS participants. While this is a known phenomenon, the number of participants who became simulator sick caused a delay in completing the ACS study. This should be taken into consideration for future studies in order to allow for some level of attrition due to simulator sickness. For the ACS study, 12 participants were unable to continue due to simulator sickness; this represents a 20 percent attrition rate in recruited ACS participants. It should be noted, however, that the mean age of these participants was almost 50 years. This may have had some influence on their likelihood of experiencing simulator sickness.

Additionally, in addition to planning for attrition due to simulator sickness newer methods of predicting simulator sickness (see Golding, 2006) should be considered by future research.

\subsubsection{Per formance Factors}

Several participants displayed a substantial drop in performance at follow-up testing as compared to their results from entry-level testing. However, a further examination revealed that many of these participants drove autoshift or synchronized transmissions at their place of employment. These participants struggled to successfully drive a double-clutching, non-synchronized, transmission in follow-up testing. This factor should be taken into consideration, especially as the number of trucks equipped with these types of transmissions increases. Several large truck load carriers now employ autoshift transmissions. Approximately 20 percent of the trucks found in the United States are now equipped with these autoshift transmissions. This also lead to a potential Informal Group participant not being eligible for participation as he was taught to drive a tractor trailer, and performed his CDL test, with an autoshift transmission.

\subsubsection{Overall CDL Throughput}

CDL throughput was a limiting factor in recruitment for the Informal Group. Several factors relevant to this became apparent during the course of this study.

Due to the economic downturn occurring during this study, employers became much more selective in hiring drivers. Several large carriers ceased hiring entry-level drivers, and only hired experienced drivers. Thus, more potential entry-drivers may be seeking some form of training. The converse of this may be occurring; in some instances entry-level drivers are seeking other types of employment. 
Two other problems encountered were potential Class A CDL participants who scheduled testing with the DMV and either did not show for their scheduled testing time or failed to show in a tractor trailer. Inquiries to the DMV relating to those not showing for their scheduled test time tend to show that the majority of these potential Class A CDL drivers are unable to find a tractor trailer to use for testing. Those not showing in a tractor trailer typically came to the test in a straight truck or a straight truck pulling a small trailer.

Data was obtained from the Delaware DMV regarding CDL throughput. There are four DMV locations in the State of Delaware; each location provides testing four days per week and has the ability to test three people per day. Accounting for holidays, a potential total of 2,437 tests may be scheduled. However, the Delaware DMV cancels testing in inclement weather such as heavy rain and snow. For the calendar year 2007, a total of 676 drivers were issued a CDL. For the calendar year 2008, a total of 549 drivers were issued a CDL. It is important to note that the Delaware DMV currently does not track whether these drivers received a Class A, Class-B, or Class-C CDL. Additionally, the above totals do not include the participants from the Simulator and Conventional Groups.

Of the total potential CDL tests per year, only 28 percent of those potential test times resulted in a driver obtaining a CDL in 2007 while dropping to 23 percent in 2008. The Delaware DMV provided weekly test schedules. Table 75 shows a 9-month period from the Georgetown location. As shown, the majority of test takers were testing for a Class-B CDL.

Table 75. Georgetown DMV CDL throughput from July 2008 to March 2009

\begin{tabular}{|l|r|r|r|r|r|r|r|r|}
\hline Category & Unsure & $\begin{array}{c}\text { None } \\
\text { Scheduled }\end{array}$ & $\begin{array}{c}\text { Class A } \\
\text { No } \\
\text { Show }\end{array}$ & $\begin{array}{c}\text { Class A } \\
\text { Not } \\
\text { Eligible }\end{array}$ & $\begin{array}{c}\text { Class A } \\
\text { No Tractor } \\
\text { Trailer }\end{array}$ & $\begin{array}{l}\text { Class A } \\
\text { Eligible }\end{array}$ & $\begin{array}{r}\text { Class-B } \\
\text { Eligible }\end{array}$ & $\begin{array}{r}\text { Class-C } \\
\text { Eligible }\end{array}$ \\
\hline Frequency & 21 & 170 & 35 & 8 & 10 & 16 & 127 & 0 \\
\hline Percent & $5.43 \%$ & $43.93 \%$ & $9.04 \%$ & $2.07 \%$ & $2.58 \%$ & $4.13 \%$ & $32.82 \%$ & $0.00 \%$ \\
\hline
\end{tabular}

This trend follows for all four DMV locations in Delaware. PTDI also provided national throughput numbers from all PTDI certified training programs and, in calendar year 2007, 16,304 drivers received a Class A CDLs from PTDI certified training programs. However, in calendar year 2008 this number dropped to 15,169, a nearly 7 percent decline.

The factors outlined above had a major effect on the recruitment of the Informal Group participants and could not have been predicted by the researchers. Unfortunately, the economic downturn coupled with the decrease in people attempting to obtain a Class A CDL led to much lower numbers of Class A drivers than would have been available as little as two years ago. While the researchers attempted multiple strategies to increase participant recruitment there were simply few informal training group participants available to recruit. 


\section{CONCLUSIONS AND FUTURE STEPS}

The FMCSA is dedicated to reducing both the incidence and severity of CMV crashes on U.S. highways. In support of this, the FMCSA commissioned the research team to examine the use and effectiveness of driving simulation as a training tool for entry-level CMV drivers, as well as to examine the advanced capabilities of the driving simulator for simulating emergency maneuvers and extreme situations. The present study presents findings of this examination.

This project commenced in August 2006, and collected training and performance data from 107 entry-level CMV drivers as they earned their Class A CDLs and began to drive in a professional capacity. These participants were drawn from four different types of training (conventional 8week training, conventional 8-week training using a simulator for approximately 60 percent of driving experience, informal training, and CDL-focused short courses). This data set allows for the comparison of drivers' DMV CDL test performance, as well as performance in BTW and simulator testing.

The study was organized to address the following topic areas: training and DMV test performance, the validity of a driving simulator for training and testing, longitudinal effects of different training methods, the effects of personality factors on training outcomes, and the advanced capabilities of the simulator for simulating emergency maneuvers and extreme events.

No differences in DMV road tests were observed based on training type; however, conventional training group participants performed better on DMV range tests than did Informal and CDLfocused participants. However, performance on the (independently validated) DTCC and Simulator road and range tests did differ by training type; with the Conventional and simulator training groups outperforming the Informal and CDL-focused Training Groups. This is an important comparison for two reasons. First, the DTCC and Simulator road and range tests were constructed as equivalent measures as compared to the DMV tests. Since the scoring on DTCC and Simulator tests was not biased by group membership, other explanations for these differences in outcomes must be explored (e.g., differences in scoring criteria, or the need for scoring standards). Second, the participants who received PTDI-certified training (involving more training time) typically outperformed those who received less total, or less structured, training.

No differences in longitudinal measures of performance, safety, self-ratings, or supervisory ratings were observed. No substantive differences in any of the real world road or range tests were present between conventionally- and simulator-trained participants. These findings indicate that a CMV driving simulator may be used to train entry-level drivers. However, testing all drivers on a CMV driving simulator does not appear to be feasible, as participants without prior experience with the simulator tend to have lower performance on simulated versions of road and range tests. Further, participants trained on the simulator had different performance between simulated and real truck versions of the same road and range test maneuvers. These findings do not yield support for the use of a simulator as a CMV driver testing platform at the current point in simulator technology. 
Advanced simulator capabilities to replicate driving under extreme driving conditions and emergency maneuvers under different vehicle cargo/load configurations were assessed. Results indicate that the simulator provides a realistic recreation of the tested scenarios.

A cost analysis was performed examining cost differences among training types. Few differences between conventional and simulator training were present. Additionally, simulator training is expected to decrease in price due to technological advancements and increases in scale of use. This report also details the findings of a case study of existing implementations of CMV simulator training that indicated the benefits and non-benefits of simulated training as well as drivers' overall favorable opinions of simulation-based training. This research effort holds implications for the future of simulation-based CMV driver training.

A summary of the main findings for this research effort are presented next. 


\subsection{KEY FINDINGS}

Main study findings are detailed, by topic area, in Table 76.

Table 76. Main Study Findings

\begin{tabular}{|c|c|}
\hline Topic Area & Major Findings \\
\hline $\begin{array}{l}\text { Training Method and } \\
\text { CDL/Related Test } \\
\text { Performance (Research } \\
\text { Question 1) }\end{array}$ & $\begin{array}{l}\text { There are no training group differences in DMV road tests, and few } \\
\text { group differences in DMV range tests. } \\
\text { - There are differences between groups on DTCC and Simulator } \\
\text { versions of DMV tests, suggesting that more training leads to greater } \\
\text { performance on these tests. }\end{array}$ \\
\hline $\begin{array}{l}\text { Validity of Simulation } \\
\text { Training and Testing } \\
\text { (Research Question 2) }\end{array}$ & $\begin{array}{l}\text { - Only moderate to low correlations between Simulator and DTCC tests } \\
\text { are present across all groups. } \\
\text { - Simulator-trained participants demonstrated significant, but moderate, } \\
\text { correlations between Simulated and DTCC tests. } \\
\text { - The simulator may be used to train many aspects of road and range } \\
\text { driving, however it cannot be used for testing those aspects at the } \\
\text { present time. } \\
\text { - There are no differences in real-world vehicle lateral control between } \\
\text { Conventional and Simulator training group participants, this is } \\
\text { evidence that the simulator is a valid method of training entry-level } \\
\text { CMV drivers. } \\
\text { - Participants trained in a PTDI-certified program have better vehicle } \\
\text { lateral control over a vehicle than participants not trained in a PTDI- } \\
\text { certified manner. }\end{array}$ \\
\hline $\begin{array}{l}\text { Longitudinal Effects of } \\
\text { Training Methods (Research } \\
\text { Question 3, 4, 5, \& 6) }\end{array}$ & $\begin{array}{l}\text { - Most participants had generally high self-ratings of control and safety } \\
\text { skills, and would recommend their manner of training to others } \\
\text { interested in learning to drive a CMV. } \\
\text { - Both Simulator and DTCC follow-up road and range test scores were } \\
\text { lower for all groups as compared to entry-level final tests. } \\
\text { - No differences in safety records were able to be determined. } \\
\text { - No differences in supervisory ratings were found. }\end{array}$ \\
\hline $\begin{array}{l}\text { Personality Correlates } \\
\text { (Research Question 7) }\end{array}$ & - Personality does not predict performance on road and range tests. \\
\hline $\begin{array}{l}\text { Advanced Simulator } \\
\text { Capabilities (Research } \\
\text { Question } 8 \text { \& 9) }\end{array}$ & $\begin{array}{l}\text { - ACS participants generally rated the scenarios as realistic. } \\
\text { - Million milers responded to simulated events appropriately more often } \\
\text { than non-million milers. }\end{array}$ \\
\hline $\begin{array}{l}\text { Cost Effectiveness Analysis } \\
\text { of Simulator-Based Training }\end{array}$ & $\begin{array}{l}\text { - Simulator training is associated with a small (\$35/participant) cost } \\
\text { savings over Conventional training. } \\
\text { - Simulator training costs are likely to decrease over the foreseeable } \\
\text { future due to decreases in the cost of simulators. }\end{array}$ \\
\hline $\begin{array}{l}\text { Case Study of Existing CMV } \\
\text { Simulator Training Programs }\end{array}$ & $\begin{array}{l}\text { Carriers are successfully using CMV simulators to train entry-level } \\
\text { drivers and provide refresher training for existing drivers. } \\
\text { - Drivers have positive opinions regarding simulation-based training. }\end{array}$ \\
\hline
\end{tabular}




\subsection{RECOMMENDATIONS FOR IMPLEMENTING SIMULATOR-BASED TRAINING BASED ON CASE STUDY FINDINGS}

One of the most important aspects of this research effort was the value input obtained from the focus group recommendations. Based on the results from the two focus group meetings, several recommendations for implementing truck simulator training programs in CDL training were apparent, including:

Better pre-training briefings that show a clear integration of the simulator-based training with carrier safety management techniques and training policies.

Carriers should portray truck simulator training as a positive, proactive approach to safety.

Carriers should offer drivers the opportunity to have "exploratory training” at their discretion. This can include informal training requests, as well as providing virtual skidpad time.

Ensure that trainers' feedback and coaching takes advantage of the record and playback capabilities of the simulator during training exercises.

Provide a variety of training scenarios that are continuously updated and/or revised to reduce familiarity.

The truck simulator hardware and software should mimic the power units and trailers used by each driver.

Although the truck simulator's visual fidelity is important, other visual aspects of a real truck, such as larger blind spots (requiring head movements) and poor visibility should be included in the simulations.

As participants believed that experiencing simulated scenarios that paralleled their actual driving environment provides the greatest benefit, scenarios should replicate each driver's current or upcoming driving environment (especially in the modeling of terrain and traffic). Scenarios should introduce drivers to challenges within a familiar driving environment.

Participants indicated that repetition of simulated training scenarios was a powerful method to ensure transfer-of-training.

Participants reported that the truck simulator had many unexplored capabilities and was being underutilized in its current format. Researchers and vehicle-simulation companies should explore other training uses of the simulator, including fatigue management, misrouting, and driver distraction risks.

\subsection{FUTURE STEPS}

Truck simulation technology continues to expand at a rapid pace, enabling many new lines of research into CMV driver behavior and training. Truck simulation has demonstrated considerable benefits in the training of entry-level CMV drivers and in skills-maintenance for experienced CMV drivers. As simulation technology in CMV operations matures and becomes more prevalent in training programs, some key areas that deserve further exploration are noted below. 
Further exploration of these issues will aid in the proper implementation of truck simulator-based training programs.

Is there a need for standardized entry-level driver training during truck simulator driving scenarios? Providing a clear set of objectives and training goals will ensure that the simulator is used to its full potential. This will also provide for instructor consistency, give the trainee clear objectives and create a more serious training environment, and ensure that the simulator is not used as a video game.

Is there a need to establish minimum hours of training, either in a simulator or BTW setting, for entry-level CMV drivers? Minimum defined standards would assist employers, training schools, and other agencies in determining what type of simulator would best fit their needs in terms of training and efficiency.

Should hours logged training in a simulator be counted as equivalent to hours logged training BTW? The PTDI currently recognizes simulator time as classroom/laboratory time, and not as the equivalent of BTW time. Further investigation of the benefits and consequences of allowing trainees to log simulator training time in the same manner as BTW time is warranted.

What are the "best practices" to ensure transfer-of-training between a truck simulator and a real truck? These "best practices" would present clear and concise guidelines or "tips" to assist employers, training schools, simulator manufacturers, and other agencies. The compilation of these practices would act as a quick reference guide.

What is the optimal amount of training time for truck simulator-based refresher training to ensure transfer-of-training to a real truck? Evaluating and providing this time would assist employers in their assessment and refresher training of drivers.

What is the optimal ratio of training time in a truck simulator and in a real truck during entrylevel driver training? An optimal ratio of simulator time and truck time will provide for a more efficient training environment, therefore allowing more instruction time with the students. Determining that optimal time will greatly enhance the usability of the simulator and allow specific scenarios, objectives, and procedures to be standardized, which will assist in training consistency in the future.

In addition, several other aspects should be investigated to improve the overall licensing process for CMV drivers and the training programs offered to these drivers.

Is there a benefit to providing standardized training to CDL licensing examiners? Currently, not all examiners have a CDL. Providing the experience, basic CMV knowledge, and background on what some driving skills entail would assist examiners in their assessment.

How can experienced CMV drivers benefit from simulation-based training of advanced simulator capabilities? An effective advanced capabilities module based on the simulator ACS findings should be developed. This training module should be evaluated closely to ensure that scenarios and scenario guidelines could benefit a myriad of training programs and institutions. Naturalistic data could be collected to assist in the development of this training module and to evaluate the benefits for those receiving the advanced capabilities 
training against those who do not receive this training. This would assist with training consistency in the future.

How can the simulator be used to deliver targeted defensive driving training? Based on naturalistic data scenarios and LTCCS scenarios that are most often repeated, a defensive driving and hazard perception training module could be developed. This will fill a key training need in terms of CMV driver refresher training and skills maintenance. The ability to practice specific defensive driving and hazard perception skills is impractical in real trucks. The safety aspect of the simulator can provide drivers with the chance to sharpen their skills in handling safetycritical events such as potential rollover situations and evasive maneuvers. 


\title{
APPENDIX A-PARTICIPANT SCREENING PROTOCOLS \\ Simulator Orientation Pre-Screening Questionnaire SimVal Phase II
}

\author{
Participant \#: $\quad$ Age: $\quad$ Gender: Male / Female
}

\section{PART 1:}

1. Do you have or have lingering effects from heart condition, stroke, or brain tumor?

YES or NO If yes, please describe:

2. Do you have or have lingering effects from a head or neck injury?

YES or NO If yes, please describe:

3. Do you suffer from epileptic seizures?

YES or NO

4. Do you have any respiratory disorders?

YES or NO If yes, please describe:

5. Do you have dizziness, vertigo, or balance problems?

YES or NO

6. Do you have problems with claustrophobia?

YES or NO If yes, please describe:

7. Are you currently taking any medications that might affect your driving?

YES or NO

8. If female, are you currently pregnant?

YES or NO 


\section{PART 2:}

Please answer the following statements using the accompanying scale.

0

1

2

3

Never

Occasionally

Often

Always

\begin{tabular}{|l|l|}
\hline \multicolumn{1}{|c|}{ Simulator-Sickness Criteria } & RATING \\
\hline $\begin{array}{l}\text { The inability to sit close to a movie screen or watch the movement of a train or } \\
\text { carnival ride without nausea, dizziness, or headaches }\end{array}$ & \\
\hline Frequent, and sometimes daily, pressure headaches & \\
\hline Nausea, headache, or dizziness on playground equipment & \\
\hline Car sickness & \\
\hline $\begin{array}{l}\text { Fatigue, headaches, nausea, or dizziness when reading or sitting in front of a } \\
\text { computer screen }\end{array}$ & \\
\hline Nausea, headache, or dizziness from riding roller coasters & \\
\hline $\begin{array}{l}\text { Inability to watch rapid movement on a TV or movie screen without getting } \\
\text { nausea or dizziness }\end{array}$ & \\
\hline Nausea, headache, or dizziness when playing video games & \\
\hline Seeing the floor or ground as being tilted or wavy & \\
\hline $\begin{array}{l}\text { Tendency to become nauseated or dizzy when turning, spinning, or doing } \\
\text { rapid movements }\end{array}$ & \\
\hline Feeling that something inside the body is in constant motion & \\
\hline Sensation that print floats at times when reading & \\
\hline
\end{tabular}




\section{Commercial Motor Vehicle Driving Simulation Validation Study Phase II Participant Information \& Screening Form: Groups 1-2}

Group:

\section{General Info}

Date:

Name:

Date of Birth:

Gender: M / F

Address:

Phone Numbers: Home:

Cell:

Alternate Phone Number \#1: Home:

Cell:

Best Time to Call:

Email:

CDL Class A Learner’s Permit Expiration Date: 


\section{General Driving History}

1. When (month \& year) did you get your general driver's license?

2. What State was your general driver's license issued in?

3. What is your driver's license number?

\section{Truck Driving/Employment History \& Goals}

1. Have you previously driven any kind of truck commercially (e.g., prior Class-A or B)?

Yes No

If yes, please describe:

Company:

Type of Operation:

Types of Vehicles:

Number of Months/Years:

Number of Miles

Number of Hours:

2. Have you ever previously worked for a trucking company in a job other than driver? Yes No. If yes, describe briefly:

3. What type of truck driving job are you seeking?

a. Distance:

i. Long-haul/national (over 500 miles per trip)

ii. Short-haul/regional (between 50 and 499 miles per trip)

iii. Local-haul/delivery (less than 50 miles per trip)

iv. Don't know

b. Type of operation (select one):

i. For Hire Truckload

(Company hired to haul other company's goods, e.g., Schneider)

ii. For Hire Less-Than-Truckload (LTL)

(Company hired to haul other company's goods that are generally 10,000lbs or less, e.g., Old Dominion)

iii. Private fleet

(Company that hauls own goods, e.g., Wal-Mart)

iv. Line-haul

(Travels from terminal to terminal, no pick-up/delivery, e.g., Pitt Ohio)

v. Don't know 


\section{Heavy Farm Equipment Use}

1. Have you ever had prior experience or do you currently operate heavy farm equipment? For example: large tractors, combines, bailers, etc...

YES or NO

If yes, please describe the types of equipment operated:

2. Approximately how many years of experience in operating this type of equipment do you have? 
In addition to the orientation questions and Participant Information \& Screening Form, participants in the informal and CDL-focused training groups were asked:

What type of truck do you plan on using to take the CDL skills test? (e.g., tractor trailer)

\section{$\underline{\text { Truck Driving Training History }}$}

1. Have you gone, or are you planning to go, to a truck driver training school?

Yes No

2. If yes, please provide the following information:

a) School name \& location:

b) Describe the program [emphasis on time in training]

c) How many hours of classroom training?

d) How many hours of behind-the-wheel training?

e) Was a simulator included in the training?

f) Was a certificate awarded? Yes No

If yes, describe:

g) Have you taken any additional commercial driving courses? Yes No If yes, list courses:

3. Have you received any on-the-job training (OJT) in commercial driving? Yes No If yes, please describe (setting, instructor, number of hours/miles, type of vehicles, type of operations, name of company, etc.):

4. Have you received any other commercial driver training? This includes informal training such as a friend or relative teaching you how to drive a truck. Yes No

If yes, please describe:

Setting:

Number of Hours/miles driven:

Type of vehicle:

Instructor (e.g., friend, father, etc.): 


\section{General/Wrap-Up}

1. Is there any additional information that you can think of relating to your background and training in trucking and truck driving?

(e.g., defensive driving course, company remedial training) If yes:

In addition to the orientation questions and Participant Information \& Screening Form, participants in the Advanced Capabilities Showcase were asked:

CDL Class A License Expiration Date:

How many endorsements do you currently carry?

Please list all endorsements and the number of years for each:

School Bus Years

Tanker Years

Doubles Years

Triples Years

Hazardous Years

Other

Years 


\section{Truck Driving Employment History:}

1. How many years have you been a commercial truck driver?

2. What trucking company do you currently work for?

3. Are you a million mile driver?

4. How many at-fault accidents have you had while driving a commercial motor vehicle?

5. What type of truck driving job do you currently have?

a. Distance:

i. $\quad$ Long-haul/national (over 500 miles)

ii. Short-haul/regional (between 51 and 499 miles)

iii. Local-haul/delivery (less than 50 miles)

iv. Don't know

b. $\quad$ Type of operation (select one):

i. $\quad$ For Hire Truckload

(Company hired to haul other company’s goods, e.g., Schneider)

ii. $\quad$ For Hire Less-Than-Truckload (LTL)

(Company hired to haul other company's goods that are generally 10,000lbs or less, e.g., Old Dominion)

iii. $\quad$ Private fleet (Company that hauls own goods, e.g., Wal-Mart)

iv. Line-haul

(Travels from terminal to terminal, no pick-up/delivery, e.g., Pitt Ohio)

v. Don't know

6. $\quad$ Please describe the following:

a. Hours driven per week:

b. Miles driven per week:

c. Type(s) of trucks driven (include \# of years and \# of miles for each):

i. Van:

ii. Tanker:

iii. Doubles: 


\section{APPENDIX B-DTCC ENTRY-LEVEL TRAINING CURRICULUM}

The DTCC curriculum consists of four different classroom courses and three different driving courses, all of which require passing scores in order to earn the certificate. Listed below (Antonik \& Derrickson, 1999) are the course descriptions:

CTS $101 \quad$ Fundamentals of Motor Fleet Safety (8 weeks-Tuesday \& Thursday evenings). This course teaches safety fundamentals and essential regulatory requirements not directly related to driving. It addresses knowledge of Federal and State regulations governing commercial drivers and motor carriers. ( 3 credits)

CTS 102 Vehicle Systems and Reporting Malfunctions (8 weeks-Monday evening). This course is designed to familiarize the student with tractor trailer vehicle systems and the proper procedures for handling and reporting vehicle malfunctions. (1 credit)

CTS 103 Tractor-Trailer Operations (4 weeks—weekdays or 8 weeks—Friday evenings). This course acquaints students with tractor-trailer control systems and introduces control concepts. It teaches vehicle inspection procedures and requirements, coupling and uncoupling procedures, and shifting procedures and patterns. (2 credits)

CTS 104 Road Driving Practices (4 weeks—weekdays or 8 weeks—weekends). This course teaches the basic control skills necessary to operate tractor-trailer vehicles on the public roadways. Registered students must participate in a random drug-testing program as mandated by Federal law. (1 credit)

CTS 105 Range Driving Practices (8 weeks—weekdays or 16 weeks—weekends). This course teaches the basic control skills necessary to safely operate tractor trailer vehicles through a series of maneuvering exercises. Students will also develop shifting, backing, inspecting, and coupling/uncoupling skills. Registered students must participate in a random drug testing program as mandated by Federal law. (2 credits)

CTS $106 \quad$ Advanced Driving Operations (4 weeks—weekdays or 8 weeks—Friday evenings). This course teaches driving principles necessary for safe operation of a tractor-trailer vehicle on the highway and explores the interaction between the vehicle and the highway traffic environment. It addresses hazard perception and response as well as emergency and evasive maneuvers. (2 credits)

CTS 107 Advanced Driving Practices (4 weeks—weekdays or 8 weeks—weekends). The student learns to apply safe operating principles, perceive hazards, and operate at night in this course. Skill development and learning occurs through behind-the-wheel training. Registered students must participate in a random drug testing program as mandated by Federal law. (1 credit) 


\section{APPENDIX C-SIMULATOR ORIENTATION PROTOCOL}

\section{Simulator Sickness \& Orientation Protocol \\ SimVal Phase II Study \\ Entry Level - Groups 1 \& 2}

- Open up the IOS program. (double click the IOS icon)

- Open the Scripted Scenarios directory found on the left side of the screen.

- Next open the Sim-Sickness directory.

- Click and drag Route 1 onto the right side of the screen.

- Right click the truck icon and select advanced, and then configure to make sure settings are correct. They should be as follows:

o Conventional Tractor

o Automatic Transmission

o Cummins 350hp engine

o No trailer

- Click the play button to begin. (Drive Exercise)

- Instruct participant that no food or drink is allowed on the simulator.

- Once the scenario has loaded, orient the participant to the simulator, screens, fields of view, mirrors, etc.:

Read to participant while orienting to truck cab:

1. Please take a seat in the driver's seat.

a. Have participant fasten safety belt

b. Point out seat adjustments (height, slide bar, backrest)

c. Point out steering wheel adjustment

d. Proceed once subject is comfortable

2. The instrumentation is fully functional except for the fuel gauge and volt gauge.

a. Point out each gauge

b. The fuel gauge will remain at $3 / 4$ of a tank

c. The volt gauge will remain at 12 volts

d. Point out indicator lights, jake brake, headlight switches, parking brake, trailer brake, ignition, turn signals, and hazard lights

3. If you happen to stall the truck, you must turn the ignition switch to the off position before restarting.

4. Also, please do not use the jake brake.

a. All the way down is off

5. The fan is fully functional. Feel free to use it if you would like.

a. Point out on/off switch location

6. The engine is a Cummins 350hp 
7. The transmission is an Eaton Fuller.

a. Point out shift tower

b. It is spring loaded on both sides, will always come back to the middle slot when released

8. For this first drive you will be using an automatic transmission.

a. Explain automatic shift pattern

9. These are your west coast (real) mirrors. Feel free to make any adjustments. You will not have a trailer for this drive.

a. Point out tractor drive axle tires in rear screens

10. These are your spot or convex mirrors. They give you the wide angle view.

11. The white strip is the front edge of your hood.

a. Point out front edge of hood

12. You are parked on a two lane rural road with a posted speed of $35 \mathrm{mph}$.

a. Point out speed limit sign.

13. Speed limits are posted throughout:

a. $25-30 \mathrm{mi} / \mathrm{h}$ in towns and cities

b. $35-55 \mathrm{mi} / \mathrm{h}$ on rural roads

c. $55 \mathrm{mi} / \mathrm{h}$ on the highways and interstates

d. Most curves have yellow warning signs posted

14. You may encounter traffic (cars, motorcycles, and other trucks). You may also encounter pedestrians, animals, traffic lights, construction zones, different weather conditions along with urban and rural driving environments.

15. The route takes about 30 minutes. For this route, I will be giving route information as you go.

16. Do you have any questions?

- Using the laminated map and route directions, give verbal commands to direct the participant on the route.

- Provide feedback to the participant when first starting out about driving the simulator successfully. For example, depth perception.

- This driving route will be approximately 30 minutes. Using the spot mirror in the cab, observe the participant for any signs of discomfort. If signs are present or participant complains of feeling ill, stop the scenario immediately. Upon completion, have the participant step out of the simulator and fill out the simulator sickness post-screening questionnaire A. If the participant scores less than 40 , then he/she can continue with the next simulator drive. If the participant scores between a 40 and 50, it is at the researcher's discretion if they should continue or not. A score of 50 or higher eliminates the participant from continuing in the study. If the participant is eligible to continue, allow a 5-minute break if they want before proceeding. 
- Once the participant is ready to proceed, open the Scripted Scenarios directory found on the left side of the screen.

- Next open the Sim-Sickness directory.

- Click and drag Route 2 onto the right side of the screen.

- Right click the truck icon and select advanced, and then configure to make sure settings are correct. They should be as follows:

o Conventional Tractor

o Automatic Transmission

o Cummins 350hp engine

o No Trailer

- Click the play button to begin. (Drive Exercise)

- Once the scenario has loaded, select the environment button (sun) and set autonomous traffic to \#4 density level.

- Have the participant get back into truck cab and fasten his/her safety belt.

- Instruct participant that they are free to drive wherever they would like for 30 minutes.

1. When you are ready, start the truck and let your air pressure build. This drive will last for 30 minutes and you are free to drive wherever you like. Use this time to continue to get a feel for the tractor. You will have an automatic transmission and no trailer. Please obey all traffic laws. Do you have any questions before you begin?

- $\quad$ Set the timer for 30 minutes and have participant begin driving.

- Instruct participant to stop at any time if they are feeling ill in any way. Keep an eye on the participant and stop them if they are looking ill.

- At the end of 30 minutes, have the subject get out of the cab and fill out the simulator sickness post-screening questionnaire B. Again, if the participant scores less than 40, then they can continue with the next simulator drive. If the participant scores between a 40 and 50, it is at the researcher's discretion if they should continue or not. A score of 50 or higher eliminates the participant from continuing in the study.

- Participants who do not pass the simulator sickness screening will be placed into the conventional group.

- Thank all participants for volunteering to participate and tell them they will be notified of group assignments the following day. 


\section{Simulator Sickness \& Orientation Protocol \\ SimVal Phase II Study \\ Entry Level - Groups 3 \& 4}

- Open up the IOS program. (double click the IOS icon)

- Open the Scripted Scenarios directory found on the left side of the screen.

- Next open the Sim-Sickness directory.

- Click and drag Sim Sickness Route 1 onto the right side of the screen.

- Right click the truck icon and select advanced, and then configure to make sure settings are correct. They should be as follows:

o Conventional Tractor

o Automatic Transmission

o Cummins 350hp engine

o No trailer

- Click the play button to begin. (Drive Exercise)

- Instruct participant that no food or drink is allowed on the simulator.

- Once the scenario has loaded, orient the participant to the simulator, screens, fields of view, mirrors, etc.

Read to participant while orienting to truck cab:

17. Please take a seat in the driver's seat.

a. Have participant fasten safety belt

b. Point out seat adjustments (height, slide bar, backrest)

c. Point out steering wheel adjustment

d. Proceed once subject is comfortable

18. The instrumentation is fully functional except for the fuel gauge and volt gauge.

a. Point out each gauge

b. The fuel gauge will remain at $3 / 4$ of a tank

c. The volt gauge will remain at 12 volts

d. Point out indicator lights, jake brake, headlight switches, parking brake, trailer brake, ignition, turn signals, and hazard lights

19. The fan is fully functional. Feel free to use it if you would like.

a. Point out on/off switch location

20. The engine is a Cummins $350 \mathrm{hp}$

21. The transmission is an Eaton Fuller.

a. Point out shift tower

b. It is spring loaded on both sides, will always come back to the middle slot when released

22. For this first drive you will be using an automatic transmission.

b. Explain automatic shift pattern 
23. These are your west coast (real) mirrors. Feel free to make any adjustments. You will not have a trailer for this drive.

a. Point out tractor drive axle tires in rear screens

24. These are your spot or convex mirrors. They give you the wide angle view.

25. The white strip is the front edge of your hood.

a. Point out front edge of hood

26. You are parked on a two lane rural road with a posted speed of $35 \mathrm{mph}$.

a. Point out speed limit sign.

27. Speed limits are posted throughout:

e. 25-30 $\mathrm{mi} / \mathrm{h}$ in towns and cities

f. $35-55 \mathrm{mi} / \mathrm{h}$ on rural roads

g. $55 \mathrm{mi} / \mathrm{h}$ on the highways and interstates

h. Most curves have yellow warning signs posted

28. You may encounter traffic (cars, motorcycles, and other trucks). You may also encounter pedestrians, animals, traffic lights, construction zones, different weather conditions along with urban and rural driving environments.

29. The route takes about 30 minutes. For this route, I will be giving route information as you go.

30. Do you have any questions?

- Using the laminated map and route directions, give verbal commands to direct the participant on the route.

- Provide feedback to the participant when first starting out about driving the simulator successfully. For example, depth perception.

- This driving route will be approximately 30 minutes. Using the spot mirror in the cab, observe the participant for any signs of discomfort. If signs are present or participant complains of feeling ill, stop the scenario immediately. Upon completion, have the participant step out of the simulator and fill out the simulator sickness post-screening questionnaire A. If the participant scores less than 40, then he/she can continue with the next simulator drive. If the participant scores between a 40 and 50, it is at the researcher's discretion if they should continue or not. A score of 50 or higher eliminates the participant from continuing in the study. If the participant is eligible to continue, allow a 5 minute break if they want before proceeding.

- Once the participant is ready to proceed, open the Locations directory found on the left side of the screen.

- Under the Locations directory, open the General Purpose directory.

- Click and drag Loading Dock Station 1 onto the right side of the screen.

- Right click the truck icon and select advanced, and then configure to make sure settings are correct. They should be as follows:

o Conventional Tractor 
- 9 or 10-speed Transmission (check counter balance)

o Cummins 350-hp engine

o $48 \mathrm{ft}$ van or flatbed (check counter balance)

- Click the play button to begin. (Drive Exercise)

- Once the scenario has loaded, select the environment button (sun) and set autonomous traffic to number 4 density level.

- Have the participant get back into truck cab and fasten his/her safety belt.

- Instruct participant that they are free to drive wherever they would like for 15 minutes.

2. When you are ready, start the truck and let your air pressure build. This drive will last for 15 minutes and you are free to drive wherever you like. Use this time to get a feel for the transmission and the trailer. You will have a 10-speed transmission and a (48-ft van or flatbed) trailer. Please drive this truck/trailer combination as you would drive your own truck in the real world. Do you have any questions before you begin?

- Set the timer for 15 minutes and have participant begin driving.

- Instruct participant to stop at any time if they are feeling ill in any way. Keep an eye on the participant and stop them if they are looking ill.

- At the end of 15 minutes, stop the scenario and reset on the driving range.

- Under the Scripted Scenarios directory, open the CTS 105 directory.

- Click and drag CDL_Range_SimVal2 onto the right side of the screen.

- Right click the truck icon and select advanced, and then configure to make sure settings are correct. They should be as follows:

o Conventional Tractor

o 9- or 10-speed Transmission (check counterbalance)

o Cummins 350-hp engine

o 40 -ft van trailer

- Click the play button to begin. (Drive Exercise)

1. When you are ready, start the truck and let your air pressure build. This drive will last for 15 minutes and you are free to practice the backing skills. Use this time to practice each maneuver. You will have a 10-speed transmission and a 40-ft van trailer. Please drive this truck/trailer combination as you would drive your own truck in the real world. Do you have any questions before you begin?

- Time for 15 minutes.

- Once 15 minutes have ended have the participant stop and get out of the cab and fill out the simulator sickness post-screening questionnaire B. Again, if the participant scores less than 40 , then they can continue with the next simulator drive. If the participant scores between a 40 and 50, it is at the researcher's 
discretion if they should continue or not. A score of 50 or higher eliminates the participant from continuing in the study.

- Participants who do not pass the simulator discomfort screening will be compensated for their time.

- Participants who have passed both simulator discomfort screening questionnaires may take a 5-minute break before proceeding. 


\section{APPENDIX D-SIMULATOR SICKNESS SCREENING QUESTIONNAIRE}

Note that the same questionnaire was used for both administration instances.

\section{Simulator Orientation Post Screening Questionnaire A SimVal Phase II}
Participant \#:
Age:
Gender: Male / Female

Please answer the following statements using the accompanying scale.
0
1
2
3

None

Slight

Moderate

Severe

\begin{tabular}{|l|l|}
\hline SYMPTOM & Rating \\
\hline General Discomfort & \\
\hline Fatigue & \\
\hline Headache & \\
\hline Eye Strain & \\
\hline Difficulty Focusing & \\
\hline Increased Salivation & \\
\hline Dry Mouth & \\
\hline Sweating & \\
\hline Nausea & \\
\hline Difficulty Concentrating & \\
\hline Fullness of Head & \\
\hline Blurred Vision & \\
\hline Dizzy (eyes open) & \\
\hline Dizzy (eyes closed) & \\
\hline Vertigo & \\
\hline Stomach Awareness & \\
\hline Burping & \\
\hline
\end{tabular}

Symptoms Score: 


\section{APPENDIX E-ADVANCED CAPABILITIES EMERGENCY MANEUVERS AND EXTREME CONDITIONS OPERATIONAL DEFINITIONS}

\begin{tabular}{|c|c|}
\hline Event & What To Look For \\
\hline Rollover Avoidance 1 & $\begin{array}{l}\text { Search: Read all signs. Includes instruments in visual search. } \\
\text { Speed: Brakes and/or shifts prior to entering curve if necessary. } \\
\text { Vehicle speed is } 40 \mathrm{mi} / \mathrm{h} \text { or less. } \\
\text { Position: Holds lane position without drifting or weaving and does not } \\
\text { leave traveled portion of roadway. Touching lane lines OK. }\end{array}$ \\
\hline Rollover Avoidance 2 & $\begin{array}{l}\text { Search: Read all signs. Includes instruments in visual search. } \\
\text { Speed: Brakes and/or shifts prior to entering curve if necessary. } \\
\text { Vehicle speed is } 40 \mathrm{mi} / \mathrm{h} \text { or less. } \\
\text { Position: Holds lane position without drifting or weaving and does not } \\
\text { leave traveled portion of roadway. Touching lane lines OK. }\end{array}$ \\
\hline Deer Crossing & $\begin{array}{l}\text { Reaction: Lets off the accelerator when deer is spotted. Does not } \\
\text { induce skid. Maintains lane position. Does not swerve out of lane to } \\
\text { avoid deer. Collision is OK. }\end{array}$ \\
\hline Snow Area & $\begin{array}{l}\text { Search: Reads all signs. Includes instruments in visual search pattern. } \\
\text { Checks mirrors. } \\
\text { Speed: Reduces speed for conditions, maintains speed smoothly and } \\
\text { evenly. Does not lose traction. } \\
\text { Position: Turns lights on if not already on. Maintains lane position. }\end{array}$ \\
\hline 8\% Upgrade -Snow & $\begin{array}{l}\text { Search: Includes instruments in visual search pattern. Checks mirrors } \\
\text { for overtaking vehicles. } \\
\text { Speed: Operates engine at peak torque, } 1100-1500 \text { RPM. Downshifts } \\
\text { as necessary to maintain power. Maintains traction. } \\
\text { Position: Maintains lane position. } \\
\text { Communication: Uses } 4 \text {-ways when traveling at less than } 35 \mathrm{mi} / \mathrm{h} \text {. }\end{array}$ \\
\hline $\begin{array}{l}\text { 8\% Downgrade - } \\
\text { Snow }\end{array}$ & $\begin{array}{l}\text { Search: Includes instruments in visual search pattern. Checks mirrors. } \\
\text { Speed: In proper gear before beginning descent. Does not shift on } \\
\text { downgrade. Maintains RPM in mid to upper part of operating range, } \\
\text { 1500-2000 RPM. } \\
\text { Position: Maintains lane position. Does not lock up brakes. } \\
\text { Communication: Uses 4-ways when traveling at less than } 35 \mathrm{mi} / \mathrm{h} \text {. }\end{array}$ \\
\hline Dirt Road & $\begin{array}{l}\text { Search: Reads all signs. Includes instruments in visual search pattern. } \\
\text { Checks mirrors. } \\
\text { Speed: Reduces speed at least } 5 \mathrm{mi} / \mathrm{h} \text { below posted limit. Maintains } \\
\text { speed smoothly and evenly. } \\
\text { Position: Maintains proper lane position. }\end{array}$ \\
\hline 8\% Downgrade - Dry & $\begin{array}{l}\text { Search: Includes instruments in visual search pattern. Checks mirrors. } \\
\text { Speed: In proper gear before beginning descent. Does not shift on } \\
\text { downgrade. Maintains RPM in mid to upper part of operating range, } \\
\text { 1500-2000 RPM. } \\
\text { Position: Maintains lane position. Does not pump brakes. } \\
\text { Communication: Uses 4-ways when traveling at less than } 45 \mathrm{mi} / \mathrm{h} \text {. }\end{array}$ \\
\hline Squeeze & $\begin{array}{l}\text { Reaction: Adjusts speed and/or lane position to accommodate traffic } \\
\text { and road conditions. Checks mirrors. Uses signals when necessary. } \\
\text { Yields to merging traffic. }\end{array}$ \\
\hline Construction Zone & $\begin{array}{l}\text { Search: Reads all signs. Includes instruments in visual search pattern. } \\
\text { Checks mirrors. } \\
\text { Speed: Reduces speed to at least } 45 \mathrm{mi} / \mathrm{h} \text {. } \\
\text { Position: Moves to/stays in proper lane. Yields to merging vehicles. }\end{array}$ \\
\hline
\end{tabular}




\begin{tabular}{|c|c|}
\hline & No passing. \\
\hline Fog & $\begin{array}{l}\text { Search: Reads all signs. Includes instruments in visual search pattern. } \\
\text { Check mirrors. } \\
\text { Speed: Reduces speed to less than } 50 \mathrm{mi} / \mathrm{h} \text {. Maintains speed } \\
\text { smoothly and evenly. } \\
\text { Position: Stays in right lane. Increases following distance. Maintains } \\
\text { proper lane position. } \\
\text { Communication: Turns on lights if not already on. Uses 4-ways when } \\
\text { traveling less than } 45 \mathrm{mi} / \mathrm{h} \text {. }\end{array}$ \\
\hline Offroad Recovery & $\begin{array}{l}\text { Reaction: Adjusts speed and lane position to accommodate traffic and } \\
\text { road conditions. Checks mirrors. Did everything reasonable to prevent } \\
\text { collision and/or reduce the severity of the outcome. (May or may not } \\
\text { have collision) }\end{array}$ \\
\hline Air Pressure Loss & $\begin{array}{l}\text { Reaction: Should be continually scanning gauges and identify air } \\
\text { pressure loss. Identifies malfunction before warning alarm. Safely } \\
\text { stops vehicle and does not stop on roadway. }\end{array}$ \\
\hline Roadway Obstruction & $\begin{array}{l}\text { Reaction: Checks mirrors. Adjusts speed to account for object and } \\
\text { surrounding traffic conditions. Changes lane or uses shoulder to avoid } \\
\text { object. Does not encroach on or hit other traffic. Does not go off road. } \\
\text { Signals if changing lanes. }\end{array}$ \\
\hline Tire Blowout & $\begin{array}{l}\text { Reaction: Maintained lane position and stopped safely. Did not use } \\
\text { hard braking, stop on highway, or have collision, rollover, jackknife. }\end{array}$ \\
\hline Rain & $\begin{array}{l}\text { Search: Reads all signs. Includes instruments in visual search pattern. } \\
\text { Checks mirrors. } \\
\text { Speed: Reduces speed to at least } 50 \mathrm{mi} / \mathrm{h} \text {. Maintains speed smoothly } \\
\text { and evenly. } \\
\text { Position: Increases following distance. Maintains proper lane position. } \\
\text { Communication: Turns on lights. Uses } 4 \text {-ways when traveling less } \\
\text { than } 45 \mathrm{mi} / \mathrm{h} \text {. }\end{array}$ \\
\hline Ice & $\begin{array}{l}\text { Search: Reads all signs. Includes instruments in visual search pattern. } \\
\text { Check mirrors. } \\
\text { Speed: Reduces speed to at least } 15-20 \mathrm{~m} / \mathrm{h} \text { when entering off ramp. } \\
\text { Maintains speed smoothly and evenly. Does not brake hard. } \\
\text { Position: Maintains proper lane position. } \\
\text { Communication: Uses } 4 \text {-ways when traveling at less than } 45 \mathrm{mi} / \mathrm{h} \text {. }\end{array}$ \\
\hline Lane Cross & $\begin{array}{l}\text { Search: Does everything reasonable to prevent collision and/or reduce } \\
\text { the severity of the outcome. (May or may not have collision) } \\
\text { Speed: Adjusts speed as necessary. } \\
\text { Position: Adjusts lane position to accommodate traffic and road. Does } \\
\text { not put others or self at risk. }\end{array}$ \\
\hline RR Crossing & $\begin{array}{l}\text { Search: Directs attention to detect any hazard entering view. Scans } \\
\text { left, right, left etc. } \\
\text { Speed: Backs off throttle prior to crossing and prepares to slow or stop } \\
\text { quickly if necessary. Must not shift while any portion of the vehicle is } \\
\text { over tracks. } \\
\text { Communication: If tanker, must stop and activate 4-ways at RR } \\
\text { crossing. }\end{array}$ \\
\hline City Curve wl Traffic & $\begin{array}{l}\text { Search: Reads all signs. Includes instruments in visual search pattern. } \\
\text { Should realize tight curve and be on look out for oncoming traffic. } \\
\text { Checks mirrors. } \\
\text { Speed: Brakes and/or shifts prior to entering curve if necessary. } \\
\text { Position: Positions vehicle on the outside (high side) of the lane. } \\
\text { Yields to oncoming traffic. Tractor does not jump curb. }\end{array}$ \\
\hline Blind Entrance & $\begin{array}{l}\text { Search: Directs attention to detect any hazard entering view. Scans } \\
\text { left, right, left etc. }\end{array}$ \\
\hline
\end{tabular}




\begin{tabular}{|l|l|}
\hline & $\begin{array}{l}\text { Speed: Backs off throttle prior to blind entrance and prepares to slow } \\
\text { or stop quickly if necessary. }\end{array}$ \\
\hline & $\begin{array}{l}\text { Search: Directs attention to detect any hazard entering view. Scans } \\
\text { left, right, left etc. } \\
\text { Ball \& Pedestrian }\end{array}$ \\
$\begin{array}{l}\text { Speed: Speed should be no more than } 25 \mathrm{mi} / \mathrm{h} . \\
\text { Position: Maintains lane position but may swerve to avoid pedestrian } \\
\text { if necessary. } \\
\text { Communication: Uses horn if time permits. }\end{array}$ \\
\hline
\end{tabular}




\section{APPENDIX F-DELAWARE DMV ROAD TEST, RANGE TEST, AND RELATED MATERIALS}

\section{DEPARTMENT OF MOTOR VEHICLES RANGE TEST}

The DMV range test to obtain a Class A CDL, also known as the skills test, consists of three backing maneuvers. It should be noted that there are a total of six CDL backing maneuvers. However, the DMV range test only consists of three. Listed below are the range backing maneuvers and order in which they are tested.

100-ft. straight line backing maneuver.

Either off-set left or off-set right.

One of the following: 90-degree 40-ft. foot alley dock, conventional parallel park, or sight side parallel park.

Examinees accrue points as errors are made. Each encroachment (i.e., touching a line or cone) is scored two points. A vehicle pull-up or if the examinee exits the vehicle to look at his/her position is scored as one point. It should be noted the examinee is allowed to pull-up or exit the vehicle to look without being assessed points up to a minimum:

Straight line backing.

One free pull-up.

One free look.

All other maneuvers.

Two free pull-ups.

Two free looks.

The examinee may take as many pull-ups or looks as needed to complete the maneuver or they reach the failing score. The examinee may obtain a maximum of 12 points before failing the range test. The range test is always administered first; therefore, an examinee who fails will not be allowed to take the road test.

\section{Department of Motor Vehicles Road Test}

The DMV Class A CDL road test consists of operating a tractor trailer through a predetermined travel route. During the test, examinees are evaluated on specific skills such as left and right turns, lane changes, expressway driving, curves, railroad crossing, road side stop/start, and intersections. Each of these skills is broken down into a set of scoring criteria (i.e., traffic checks, turn signal, coasting, wide/short on turns, both hands on the wheel). Additionally, examinees are critiqued on their general driving skills (i.e., shifting, clutch use, lane deviations, braking) and for serious errors (i.e., moving violations, striking a curb while turning). It should be noted that a serious error results in an automatic failure. See the list below for the major areas of evaluation. 
4 left turns.

4 right turns.

2 stopping intersections.

2 through intersections.

Rural driving.

Urban driving.

Expressway driving.

2 left lane changes.

2 right lanes.

Left and right curve.

Road side stop/start.

Railroad crossing.

Traffic sign identification.

General driving.

Serious errors.

Including each skill section listed above; there are a total of 314 scoring criteria.

Examinees accrue points as errors are made. The examinee may obtain a maximum of 30 points before failing the road test. 
CDL Skills Tests Score Sheet (front)

\section{VEHICLE INSPECTION TEST}

${ }^{*}$ ) If vehicle has no air brakes, put a line through these components.

\begin{tabular}{|c|c|}
\hline \multicolumn{2}{|c|}{ Engine Start } \\
\hline 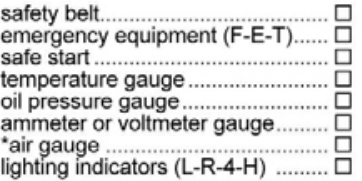 & 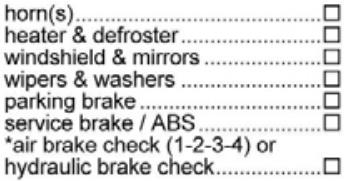 \\
\hline
\end{tabular}

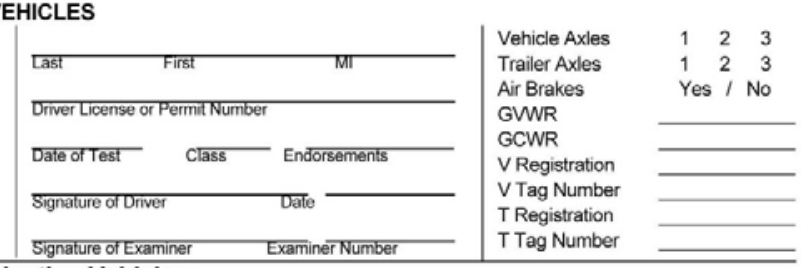

air \& electric connnectors $\quad$........... $\quad$ air \& electric connectors \& lines ....

air \& electric connnectors $\quad$........... $\quad$ air \& electric connectors \& lines ....

Coupling System

mounting bolts

pintle hook

hafere lever...

safety devices

Form

Front of Vehicle

lights \& reflectors (L-R-4.HL-HB-C) ... $\square$

Engine Compartment

coolant level........................................

power steering fluid

water pump

alternator.......................

air compressor ...................................

leaks \& hose

Steering

steering box \& hoses................... ㅁ.

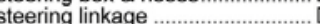

Front Axle

Front Wheel

tires (I-C-D).

rims ...

Front Suspension

springs \& shocks

spring mounts

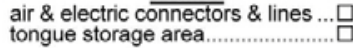

Coupling System

tongue or drawbar

mounting bolts

sliding pintle

\section{Front Brake}

brake hoses or lines

slack adjustor \& push-rod

drum \& linings or

inings or rotor \& dis

\section{Form B}

Fuel Area/Under/Side

Driver/Fuel Area

door \& mirror

fuel tank \& cap \& leaks

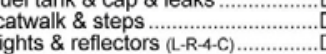

Under Vehicle

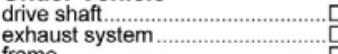

frame

\section{Rear Axles}

Rear Wheels

tires

lug nuts

axle seal

spacers or budd spacing ...............

Rear Suspension

springs \& airbags \& shocks ...........

spring/air mounts \& torque arm....

Rear Brakes

brake hoses or lines.

"brake chamber...........................

drum \& linings or rotor \& disk .........

Rear of Vehicle

oors \& ties \&

ints \& reflectors. (L....................

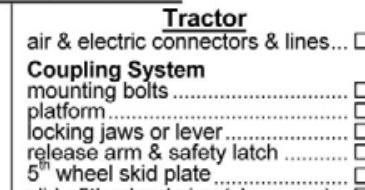

air \& electric comnectors ...............

Coupling System

kingpin

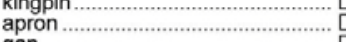

ide 5 th wheel pins (clearance)...

\section{Form C (Combinations)}

Trailer/Semi-Trailer

Trailer Front

header board or bulkhead................

lights \& reflectors (C)

Side of Trailer

landing gear......................

frame \& tandem relea

(n)........................

lights \& reflectors (L-R.4.C) ...................

Trailer Axles

Wheels

tires (I-C-D)

lug nuts

lug nuts

spacers or budd spacing...................

Suspension

springs \& air bags \& shocks.

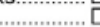

spring/air mounts \& torque arm .......

Brakes

brake hoses or lines

tor \& push-rod

drum $\&$ linings or rotor $\&$ dis

Rear of Trailer

doors \& ties \& lif

lights \& reflectors (L-R-4-TL-BL-C) /ABS.

\section{All Passenger Vehicles}

Passenger Items

passenger entry \& lift ................... $\square$

emergency exits............................ $\square$

seating...............................................

Baggage Compartment

doors secure (baggage) .............. $\square$

\section{School Bus (Only)}

\section{Student Lights}

student lights (front, back) .......... $\square$

Student Safety Items

stop arm \& safety arm ................. $\square$

student mirror

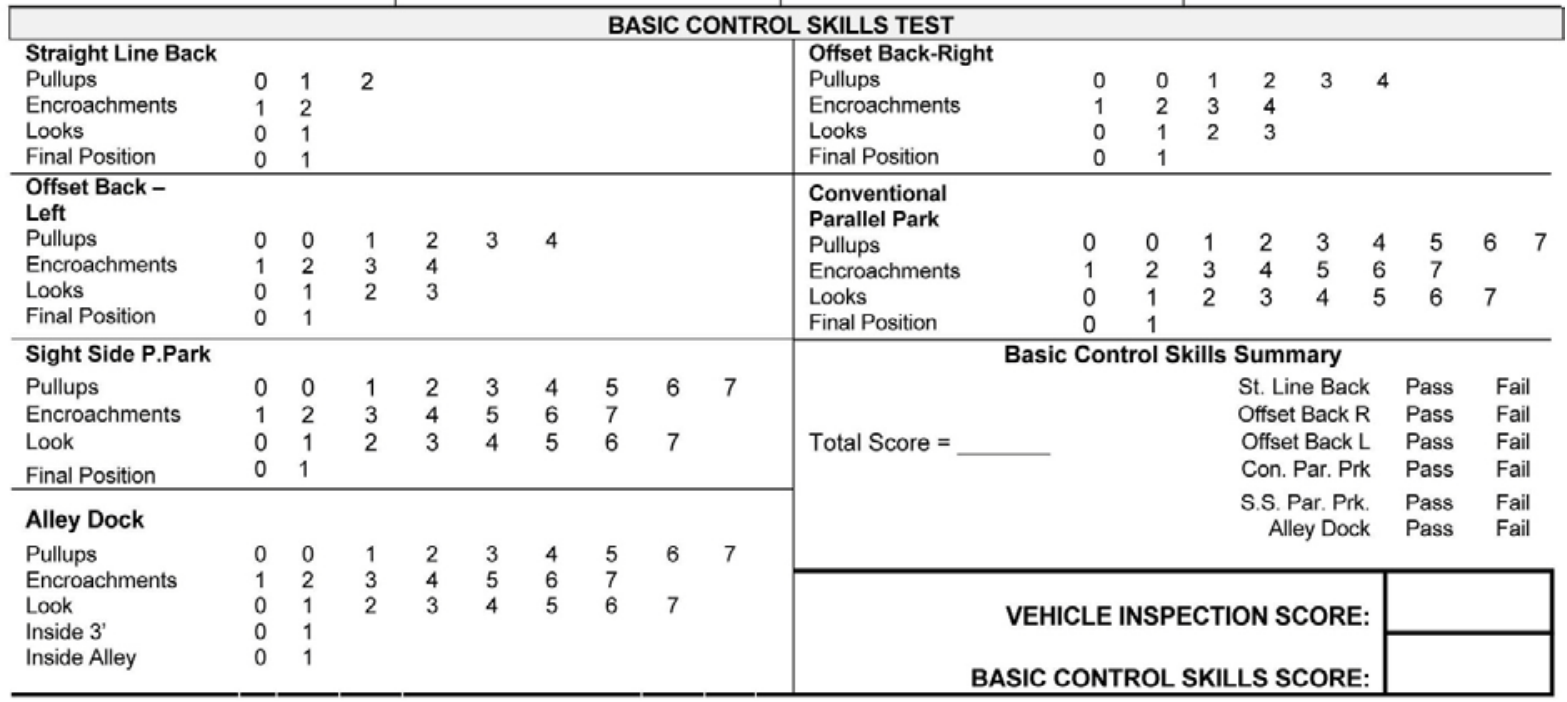

Figure 50. Photo. Delaware DMV CDL exam score sheet (first page). 


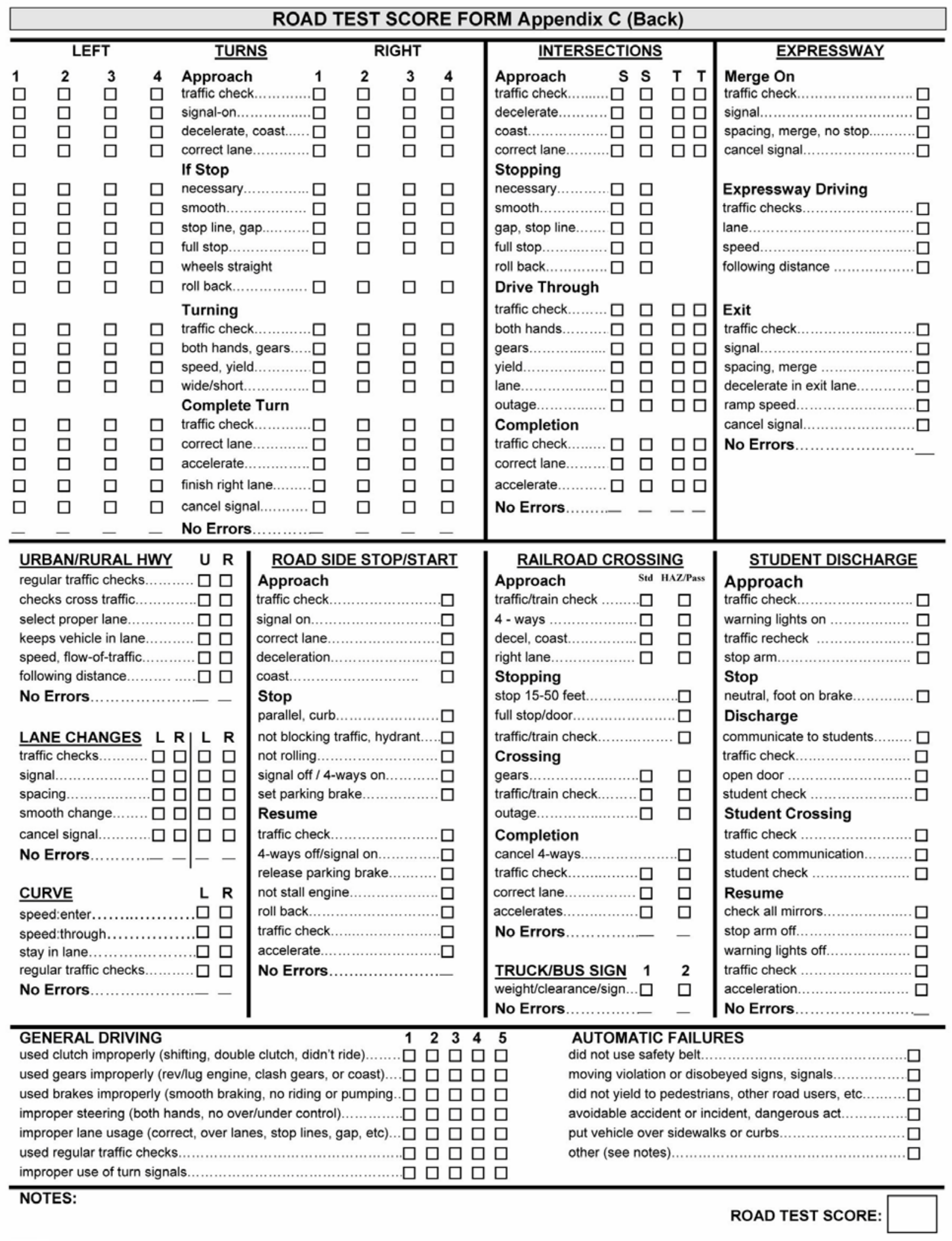

Figure 51. Photo. Delaware DMV CDL exam score sheet (second page). 
Table 77. CDL Passing Scores

\begin{tabular}{|l|l|c|c|}
\hline \multirow{2}{*}{$\begin{array}{l}\text { PRE-TRIP } \\
\text { (Total is number of correct answers) }\end{array}$} & Pre-Trip Form & \multicolumn{2}{|c|}{ VEHICLE } \\
\cline { 2 - 4 } & FORM A & 35 & 27 \\
\cline { 2 - 4 } & FORM B & 67 & 43 \\
\cline { 2 - 4 } & FORM FULL & CLASS A & CLASS B \\
\hline \multirow{2}{*}{$\begin{array}{l}\text { BASIC CONTROL SKILLS TEST } \\
\text { (Total is number of } \underline{\text { errors }} \text { ) }\end{array}$} & \multirow{2}{|}{12 points or less (all vehicles) } \\
\hline \multicolumn{2}{|l}{$\begin{array}{l}\text { ROAD TEST } \\
\text { (Total is number of errors) }\end{array}$} & 30 points or less (all vehicles) \\
\hline
\end{tabular}


[This page intentionally left blank.] 


\section{APPENDIX G-COMMERCIAL DRIVER 4-MONTH \\ FOLLOW-UP SELF-ASSESSMENT}

\section{Commercial Driver 4-Month Follow-Up Self Assessment}
Date:
Participant \#:
Group \#:

Part 1. General Evaluation

Now I'm going to ask you some questions followed by the responses you can select. Please select the response that most accurately fits your rating.

1. Compared to other drivers with equal experience (Class A drivers with about 4 months experience), how would you rate your current vehicle control and maneuvering skills (e.g., proper shifting, smooth acceleration \& braking)? (Circle one)

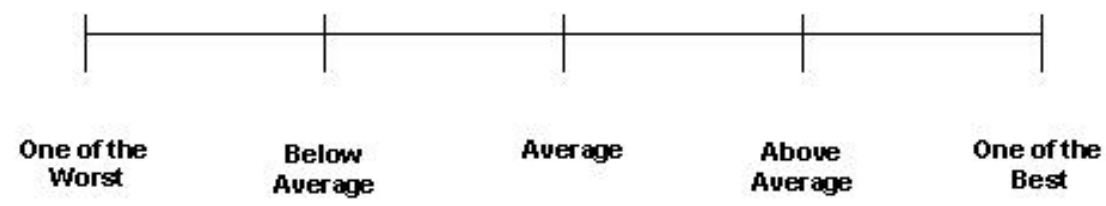

2. Compared to other drivers with equal experience (Class A drivers with about 4 months experience), how would you rate your current safe operations skills (e.g., defensive driving, speed maintenance, space management)? (Circle one)

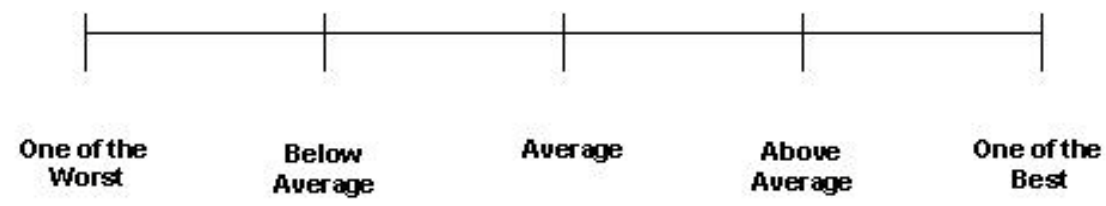

3. How would you rate the entry-level training you received in terms of teaching you vehicle control and maneuvering skills (e.g., proper shifting, smooth acceleration \& braking)? (Circle one)

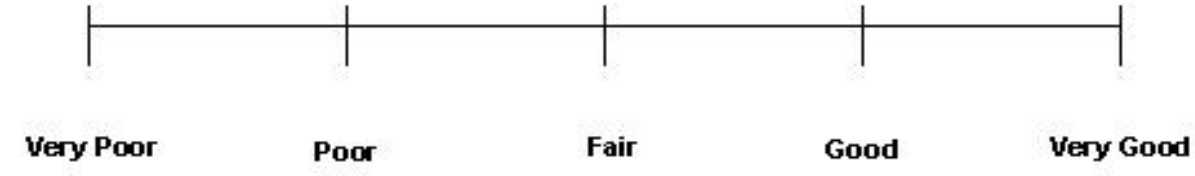

4. How would you rate the entry-level training you received in terms of teaching you safe operations skills (e.g., defensive driving, speed maintenance, space management)?

(Circle one)

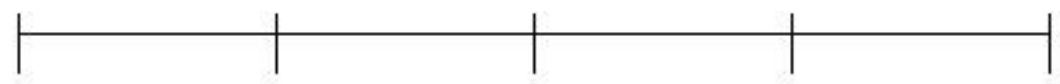


5. Looking back to when you first received your CDL, how would you rate your vehicle control and maneuvering skills (e.g., proper shifting, smooth acceleration \& braking) compared to other new CDL holders? (Circle one)

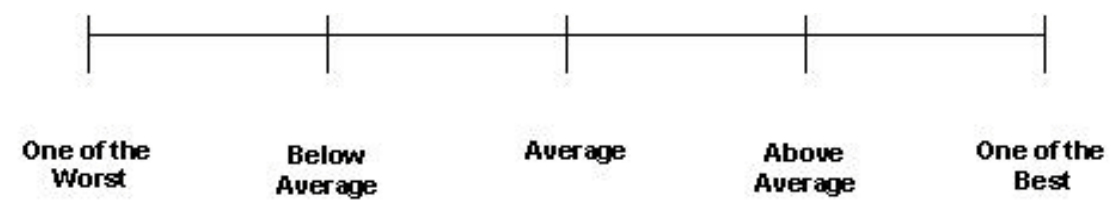

6. Looking back to when you first received your $C D L$, how would you rate your safe operations skills (e.g., defensive driving, speed maintenance, space management) compared to other new CDL holders? (Circle one)

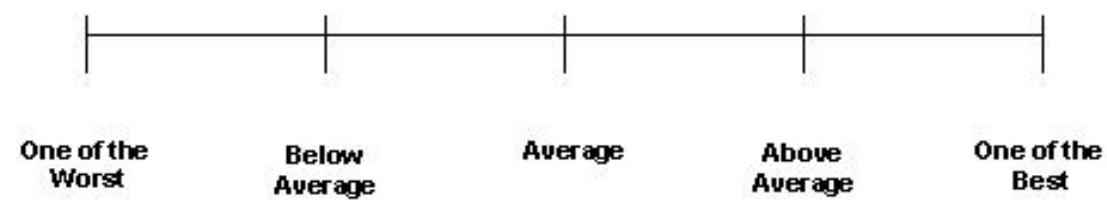

7. How do you think the amount of behind-the-wheel training you received compares to other drivers? (Circle one)

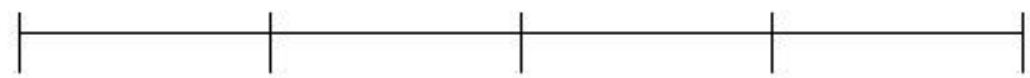

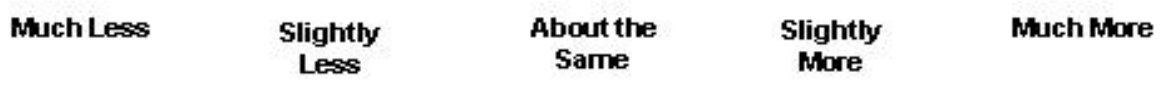

Why?

8. Would you recommend the training you received to others?

No

Yes

Why? 


\section{Part 2. Skills Tests Evaluation}

During the study you participated in you took a simulated skills test and a BTW skills test . Looking back, do you think that these tests were a fair assessment of your driving skills at that time? Please select the response that most accurately fits your rating.

1. Simulated Skills Test:

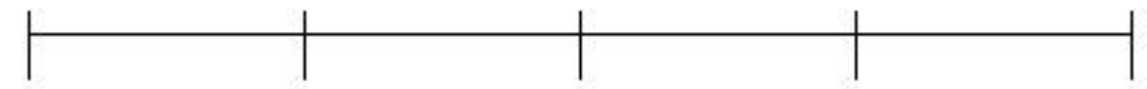

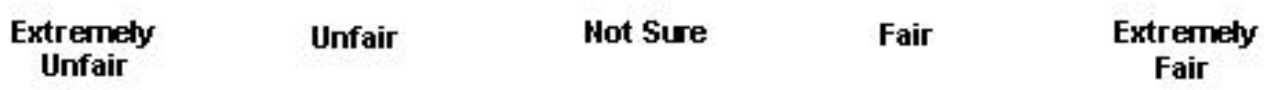

2. Behind-the-Wheel DTCC Skills Test:

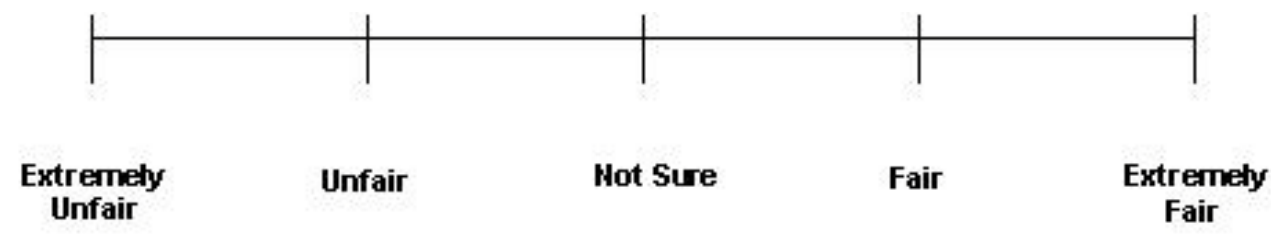

\section{Thank You!}




\section{APPENDIX H-COMMERCIAL DRIVER ON-THE-JOB SAFETY PERFORMANCE EMPLOYER QUESTIONNAIRE}

\section{Commercial Driver On-Job Safety Performance Questionnaire Safety Manager Evaluation}

Driver:

Company:

Safety Manager:

Date:

1. Driver Start Date with company (as driver):

2. Approximate miles driven since hire date:

3. Number of DOT-reportable (i.e., fatality, injury, or towaway) accidents since hire:

4. Of the above, how many were preventable (caused by driver or otherwise avoidable)?

5. Number of non-DOT-reportable accidents since hire (note: please include all non-reported impacts regardless of how minor or whether they were police-reported):

6. Of the above, how many were preventable (caused by driver or otherwise avoidable)?

7. Number of moving violations (as commercial driver) since hire:

8. Compared to other drivers with equal experience, how would you rate this driver's basic vehicle control and maneuvering skills? (Place an $\mathrm{X}$ along the scale)

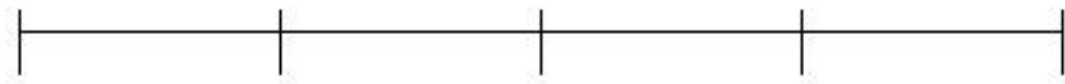

One of the
Worst $\quad \begin{gathered}\text { Below } \\ \text { Average }\end{gathered} \quad$ Average $\quad \begin{gathered}\text { Above } \\ \text { Average }\end{gathered} \quad \begin{gathered}\text { One of the } \\ \text { Best }\end{gathered}$

9. Compared to other drivers with equal experience, how would you rate this driver's safe operations (e.g., defensive driving) skills? (Place an $X$ along the scale)

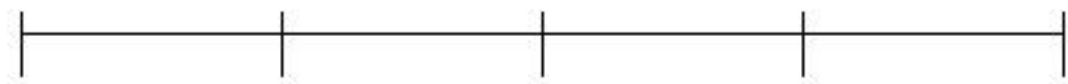

\begin{tabular}{|c|c|c|}
\hline $\begin{array}{l}\text { One of the } \\
\text { Worst }\end{array}$ & $\begin{array}{c}\text { Below } \\
\text { Average }\end{array}$ & Average \\
\hline
\end{tabular}

10. If you have any additional comments, please feel free to write them on the back or contact at

Thank you! 


\section{APPENDIX I-ADVANCED CAPABILITIES TRAINING AND DRIVING HISTORY QUESTIONNAIRE}

SimVal Phase 2

Advanced Capabilities Truck Driver Training and Driving History Questionnaire

Date:

Participant \#:

\section{Truck Driving Training History:}

1. Have you gone to a truck driver training school?

Yes No

2. If yes, please provide the following information:

a) Dates:

b) School name \& location:

c) Describe the program [emphasis on time in training]

d) How many hours of classroom training?

e) How many hours of behind-the-wheel training?

f) Was a simulator included in the training?

g) Was a certificate awarded? Yes No

If yes, describe:

h) Have you taken any additional commercial driving courses? Yes No If yes, list courses:

3. Have you received any on-the-job training (OJT) in commercial driving? Yes No If yes, please describe (setting, instructor, number of hours/miles, type of vehicles, type of operations, name of company, etc.):

4. If you did not go to a truck driver training school, please describe how you were taught to drive a truck. This includes informal training such as a friend or relative teaching you how to drive a truck. 
5. Do you have any additional comments?

\section{Truck Driving History:}

1. Have you experienced any of the following hazardous, dangerous situations during actual driving? If "yes", mark which ones. YES or NO
- Jackknife
- Tire Blowout
a Black Ice
- Blowing Snow
๑ High speed merging traffic
๑ Heavy Fog
- Heavy Rain
- Steep upgrades and downgrades
a Brake Failure
- High Speed Evasive Maneuver
- Other 


\section{APPENDIX J -ADVANCED CAPABILITIES POST-DRIVE QUESTIONNAIRE}

\section{SimVal Phase 2}

Advanced Capabilities Showcase Questionnaire

Date: Participant \#:

Trailer:

Please complete the following assessment of simulator performance.

\section{Part 1. Simulator Evaluation}

Compare the following features in the simulator to an actual tractor trailer. Place an $X$ along the scale that best represents your response.

Example:

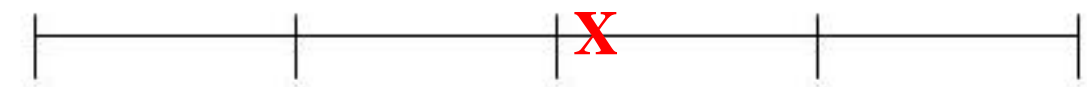

\begin{tabular}{|c|}
\hline $\begin{array}{l}\text { Extremely } \\
\text { Different }\end{array}$ \\
\hline
\end{tabular}

1. Upshifting:

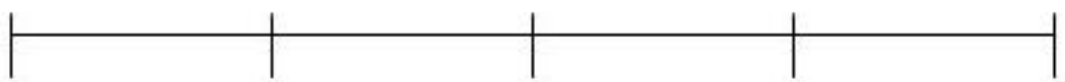

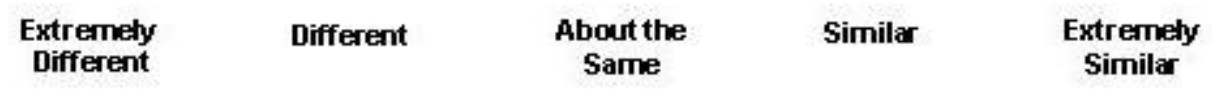

2. Downshifting:

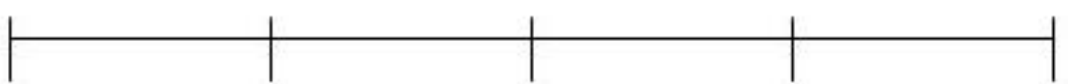

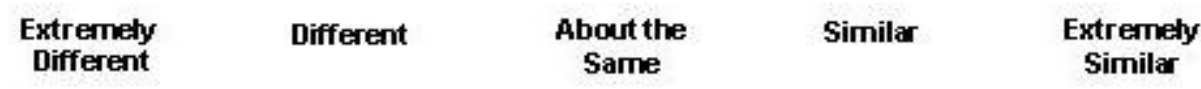

3. Acceleration:

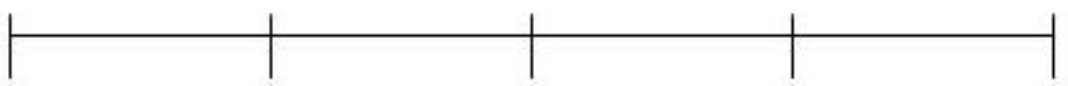

\begin{tabular}{|c|c|c|c|}
\hline $\begin{array}{l}\text { Extremely } \\
\text { Different }\end{array}$ & rent & $\begin{array}{l}\text { About the } \\
\text { Same }\end{array}$ & Similar \\
\hline
\end{tabular}

4. Braking:

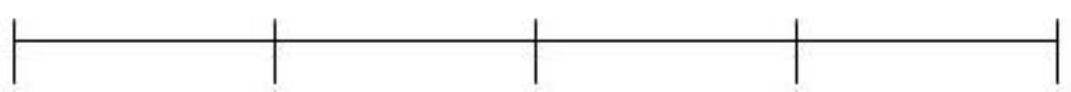

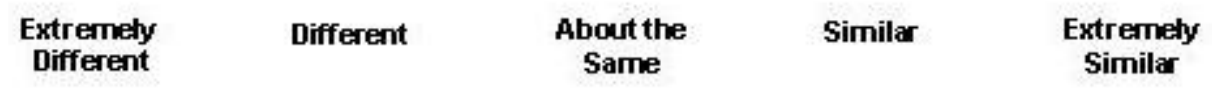


5. Steering:

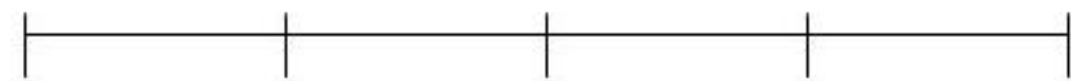

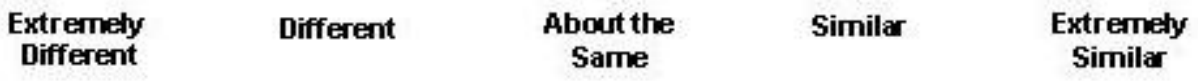

6. Turning:

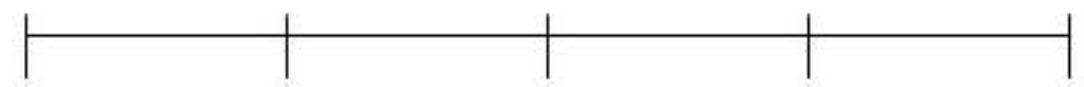

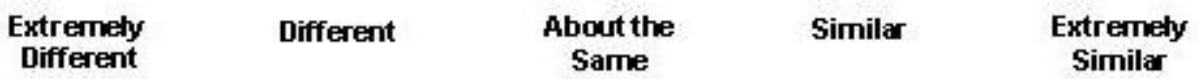

7. Handling

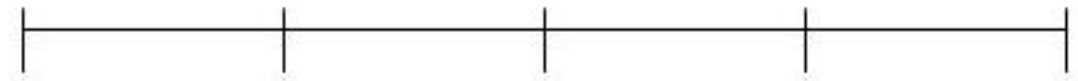

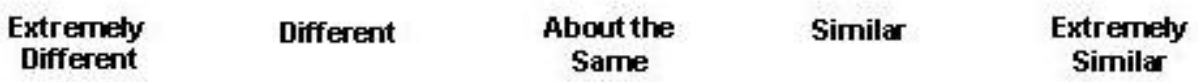

8. Mirrors:

(Views realistic)

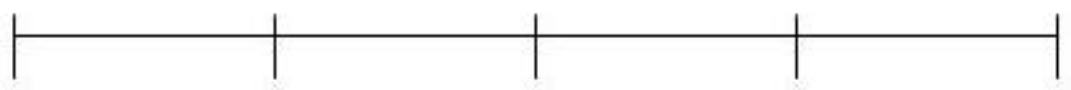

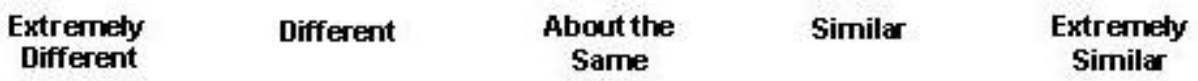

9. Trailer:

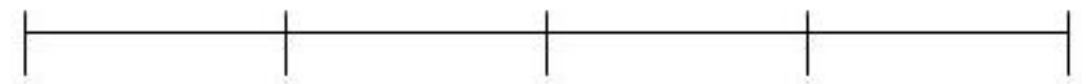

(Characteristics \& handling)

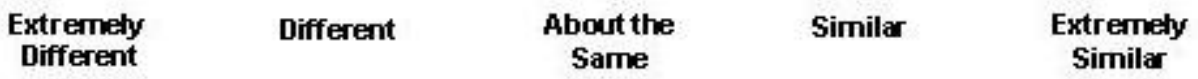

10. Visuals:

(Signs, lane

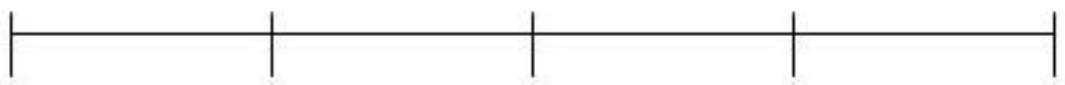
markings, road surface, other vehicles)

11. Potential Threats: (Situations you encountered)

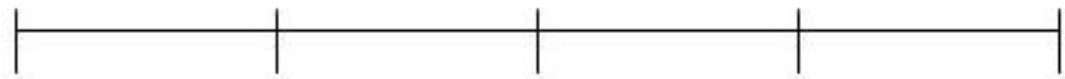

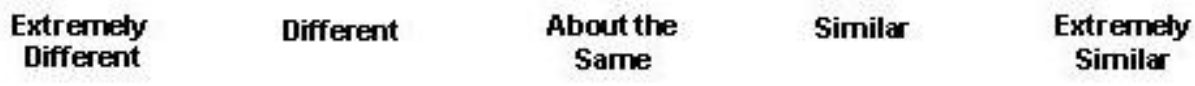

12. Overall Realism:

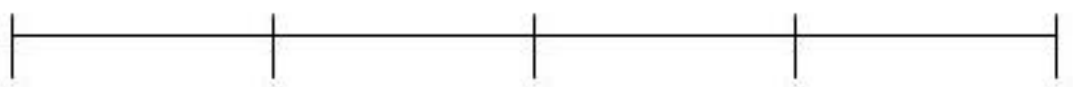

\begin{tabular}{|c|c|}
\hline Extremely & Different \\
\hline
\end{tabular}


13. Skills:

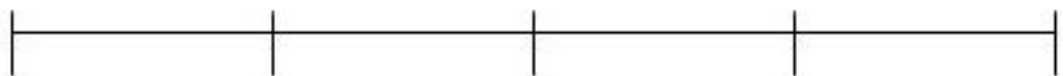

(How did you

handle the situations

encountered in the

Extremely

Different

About the

Similar

Extremely scenario?)

14. What aspect(s) of the simulator do you think were most effective? (Check all that apply and rank the top 3 among those with 1 being the most effective)

\begin{tabular}{|l|c|c|}
\hline \multicolumn{1}{|c|}{ Aspect } & $\begin{array}{c}\text { Most Effective } \\
\text { (check all that apply) }\end{array}$ & $\begin{array}{c}\text { Top 3 } \\
(\mathbf{1}, 2,3)\end{array}$ \\
\hline Safety & & \\
\hline Overhead Views & & \\
\hline Repeatability & & \\
\hline Standardization & & \\
\hline Realism & & \\
\hline Enrichment (e.g., weather, mountains) & & \\
\hline Basic Control & & \\
\hline Vehicle Handling & & \\
\hline Safe Operations & & \\
\hline Backing Skills & & \\
\hline
\end{tabular}

15. What aspect(s) of the simulator do you think were least effective? (Check all that apply and rank the top 3 among those with 1 being the least effective)

\begin{tabular}{|l|c|c|}
\hline \multicolumn{1}{|c|}{ Aspect } & $\begin{array}{c}\text { Least Effective } \\
\text { (check all that apply) }\end{array}$ & $\begin{array}{c}\text { Top 3 } \\
(\mathbf{1 , 2 , 3 )}\end{array}$ \\
\hline Safety & & \\
\hline Overhead Views & & \\
\hline Repeatability & & \\
\hline Standardization & & \\
\hline Realism & & \\
\hline Enrichment (e.g., weather, mountains) & & \\
\hline Basic Control & & \\
\hline Vehicle Handling & & \\
\hline Safe Operations & & \\
\hline Backing Skills & & \\
\hline
\end{tabular}




\section{Part 2. Event Evaluation}

How realistic were each of the following emergency/evasive maneuvers and extreme conditions that you encountered during the scenario? Place an $X$ along the scale that best represents your response.

Example:

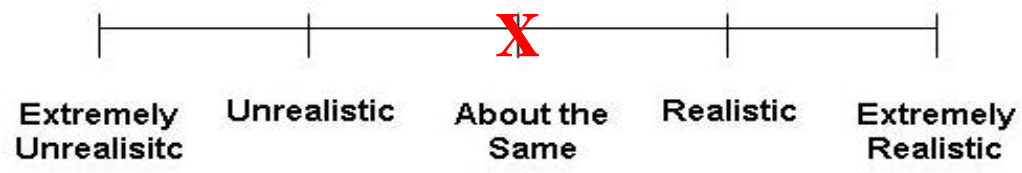

\section{Emergency/Evasive Maneuvers:}

1. Merge squeeze:

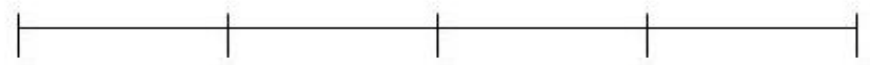

Extremely Unrealistic About the Realistic
Unrealisitc $\quad \begin{gathered}\text { Extremely } \\ \text { Realistic }\end{gathered}$

Comments:

2. Lane cross:

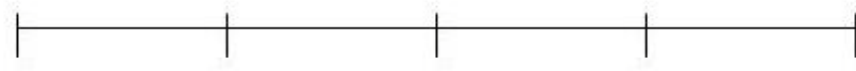

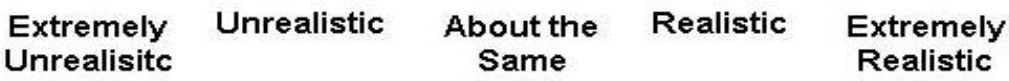

Comments:

3. Tire blowout:

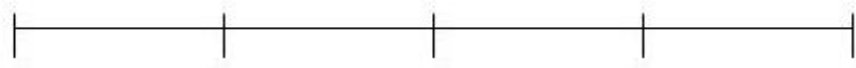

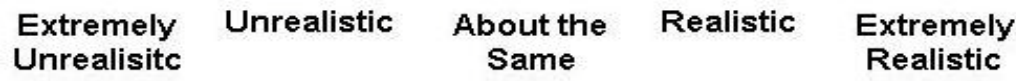

Comments:

4. Possible rollover

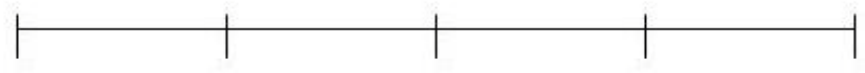

Situations (right):

Extremely

Unrealistic

About the

Realistic

Extremely Unrealisitc Same

Realistic

Comments: 
5. Possible rollover Situations (left):

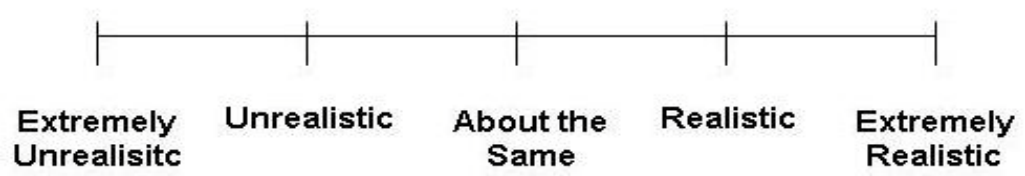

Comments:

6. Brake failure:

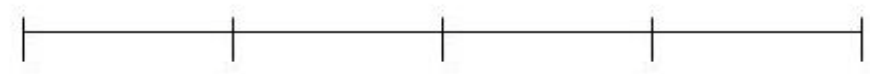

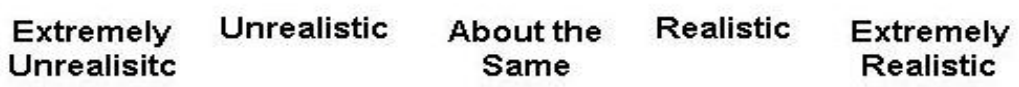

Comments:

7. Off-road recovery:

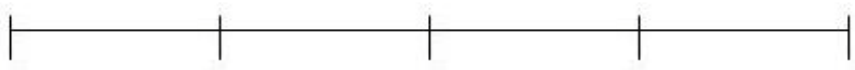

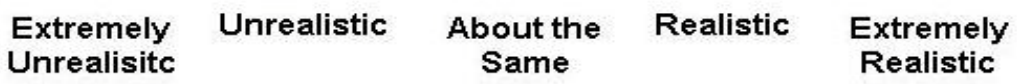

Comments:

8. Animal crossing:

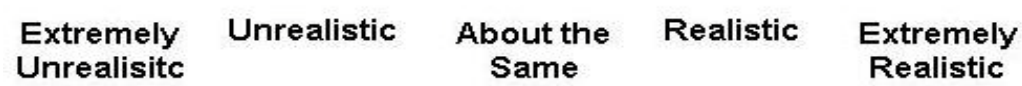

Comments:

9. Vehicle pulling out from blind entrance:

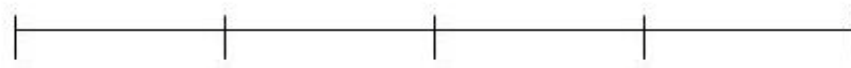

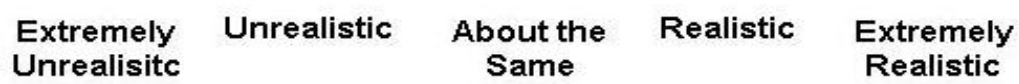

Comments:

10. Pedestrian encounter:

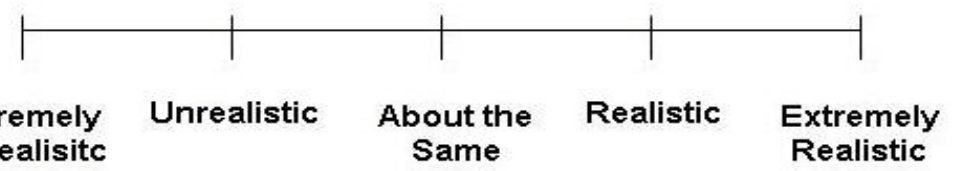

Comments: 
11. Tight city turn with trailer off tracking:

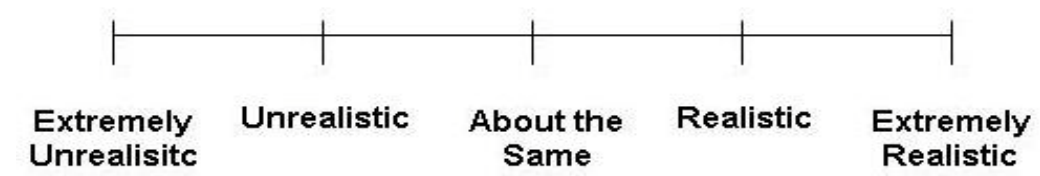

Comments:

12. Roadway obstruction:

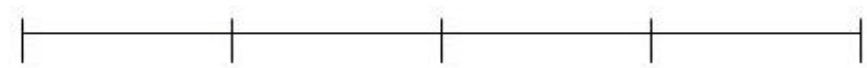

Extremely
Unrealisitc Unrealistic $\begin{gathered}\text { About the } \\ \text { Same }\end{gathered} \quad$ Realistic $\begin{gathered}\text { Extremely } \\ \text { Realistic }\end{gathered}$

Comments:

\section{Extreme Driving Conditions:}

13. Varying levels of fog:

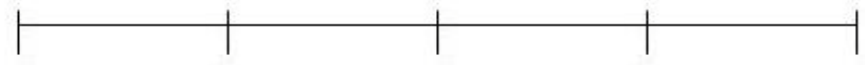

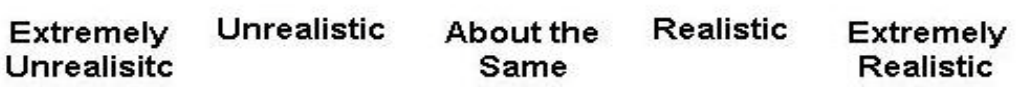

Comments:

14. Heavy rain with slick roads:

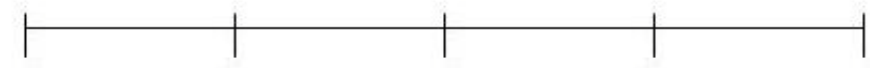

$\begin{array}{cccc}\text { Extremely Unrealistic About the } & \text { Realistic } \\ \text { Unrealisitc } & \text { Extremely } & \begin{array}{c}\text { Extre } \\ \text { Realistic }\end{array}\end{array}$

Comments:

15. Snow-covered roads:

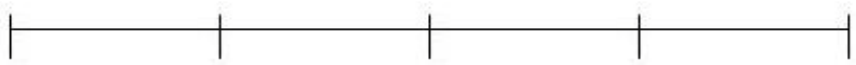

$\begin{array}{cccc}\text { Extremely Unrealistic } & \begin{array}{c}\text { About the } \\ \text { Same }\end{array} & \text { Realistic } & \begin{array}{c}\text { Extremely } \\ \text { Realistic }\end{array}\end{array}$

Comments:

16. Black ice:

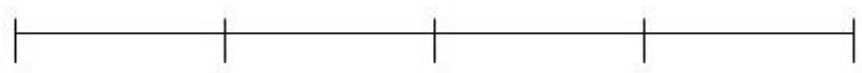

Extremely Unrealistic
Unrealisitc $\quad \begin{gathered}\text { About the } \\ \text { Same }\end{gathered}$ Realistic $\begin{gathered}\text { Extremely } \\ \text { Realistic }\end{gathered}$

Comments: 
17. Mountain stretches with 8 percent upgrades:

Extremely Unrealistic Unrealisitc

About the Realistic Same

Extremely Realistic

Comments:

18. Mountain stretched with 8 percent downgrades:

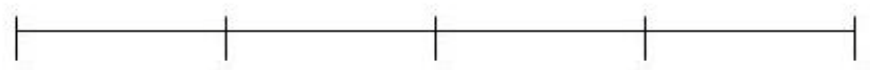

Extremely Unrealisitc

Unrealistic

About the Realistic

Extremely Same

Realistic

Comments:

19. Dirt road:

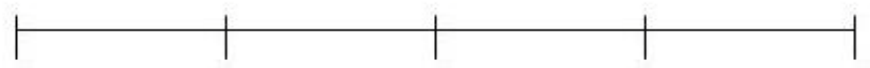

$\begin{array}{cccc}\text { Extremely Unrealistic } & \begin{array}{c}\text { About the } \\ \text { Same }\end{array} & \text { Realistic } & \begin{array}{c}\text { Extremely } \\ \text { Realistic }\end{array}\end{array}$

Comments:

20. Construction zone with one lane

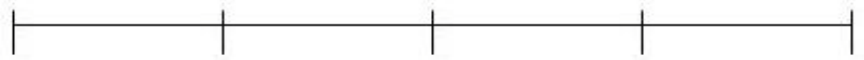
$\begin{array}{ll}\text { with one lane } & \text { Extremely } \\ \text { closed on interstate: } & \text { Unrealisitc }\end{array}$

Unrealistic

About the Same

Extremely Realistic

Comments:

21. Railroad crossing:

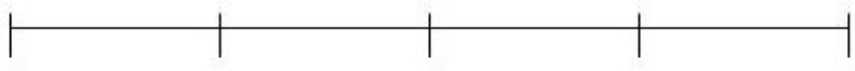
Extremely Unrealistic Unrealisitc

About the Realistic
Same $\quad \begin{gathered}\text { Extremely } \\ \text { Realistic }\end{gathered}$

Comments: 
22. What would you change about the scenarios to provide a more appropriate tool for assessing the advanced driving capabilities (e.g., different events, longer scenario, more transmission options)?

23. If you had to identify three points you like least and three points you like most about the simulator, what would they be?

\section{Least}

1.

2.

3.
Most

1.

2.

3.

24. Do you have any other comments?

\section{THANK YOU!}




\section{APPENDIX K-TEST SCORE VALIDATION RESULTS}

\section{INTRODUCTION}

In an effort to validate the test scoring, a sample of DTCC and Simulator road and range tests was selected for evaluation for each of the four training groups. Each participant in the validation sample had his/her test reviewed by the independent (outside of the research team or DTCC) reviewer, allowing for a comparison of the number of demerits assigned on the exam. The differences between the reviewer- and the DTCC instructorassigned number of demerits were calculated. The differences between reviewer and DTCC demerits on each test were assessed by group (conventional, simulator, informal, and CDL-focused training groups) and by test modality (DTCC or simulator-based testing). These validations were performed for both road and range tests.

\section{ROAD TEST VALIDATION}

A total of 162 road tests were examined by the reviewer. Descriptive statistics for DTCC demerits, reviewer demerits, and change scores are presented in Table 78. 


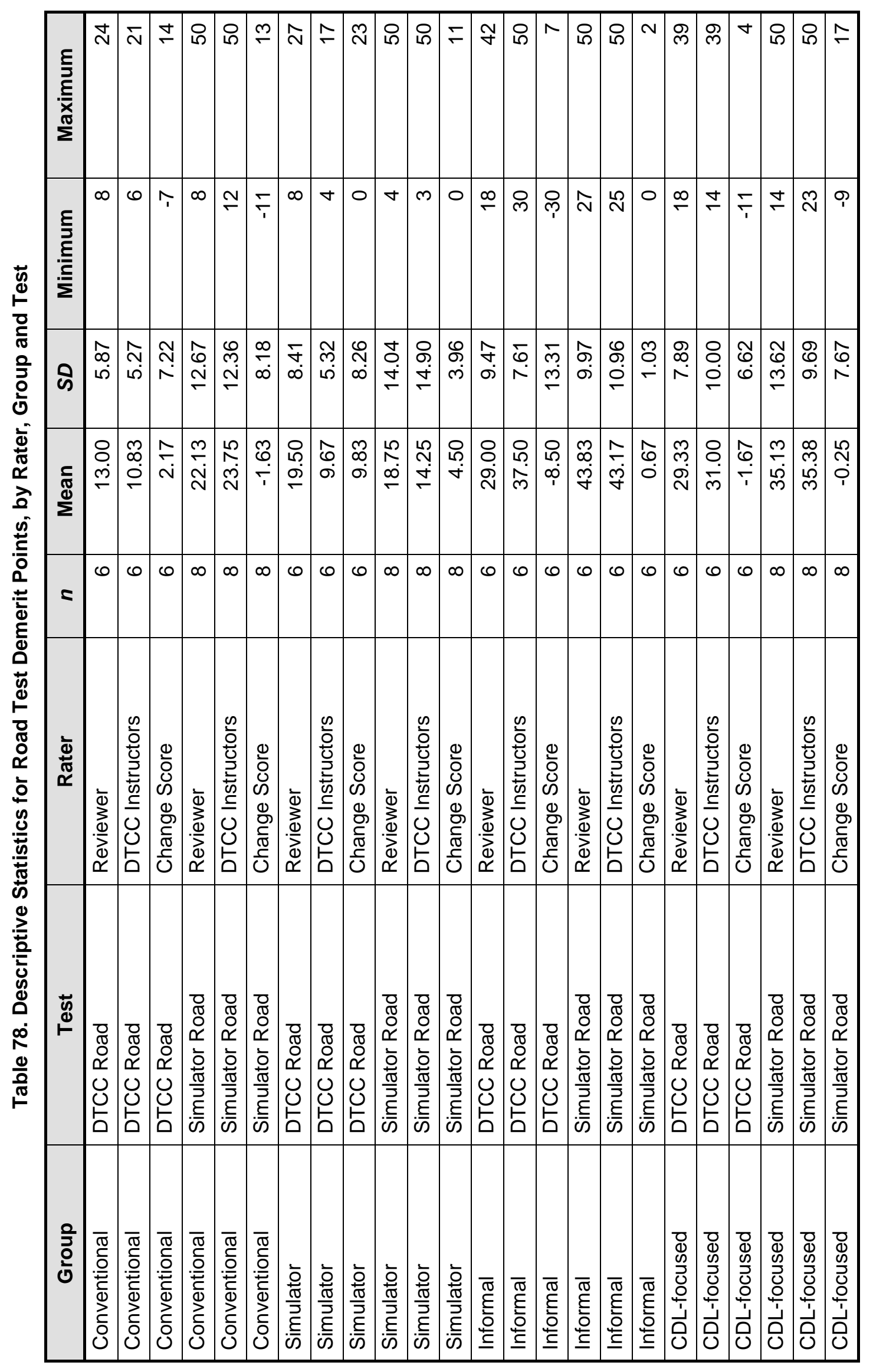


The change scores were analyzed for differences between DTCC instructor ratings and reviewer ratings by training group membership. A significant difference was present for the simulator training group $(T(13)=3.91, p=.002)$, indicating that the reviewer and DTCC instructors assigned a significantly different number of demerit points for this group. No other group comparison demonstrated statistical significance $(p>.05)$.

Further analysis of average demerits given to the simulator training group by the reviewer and DTCC personnel indicated that the reviewer assigned a significantly greater number of demerits $(M=19.07, S D=11.55)$ to participants in this group as compared to DTCC personnel $(M=12.29, S D=11.66)$. Although this is a significant difference, it should be noted that this is a 6.79 average point difference (Figure 52).

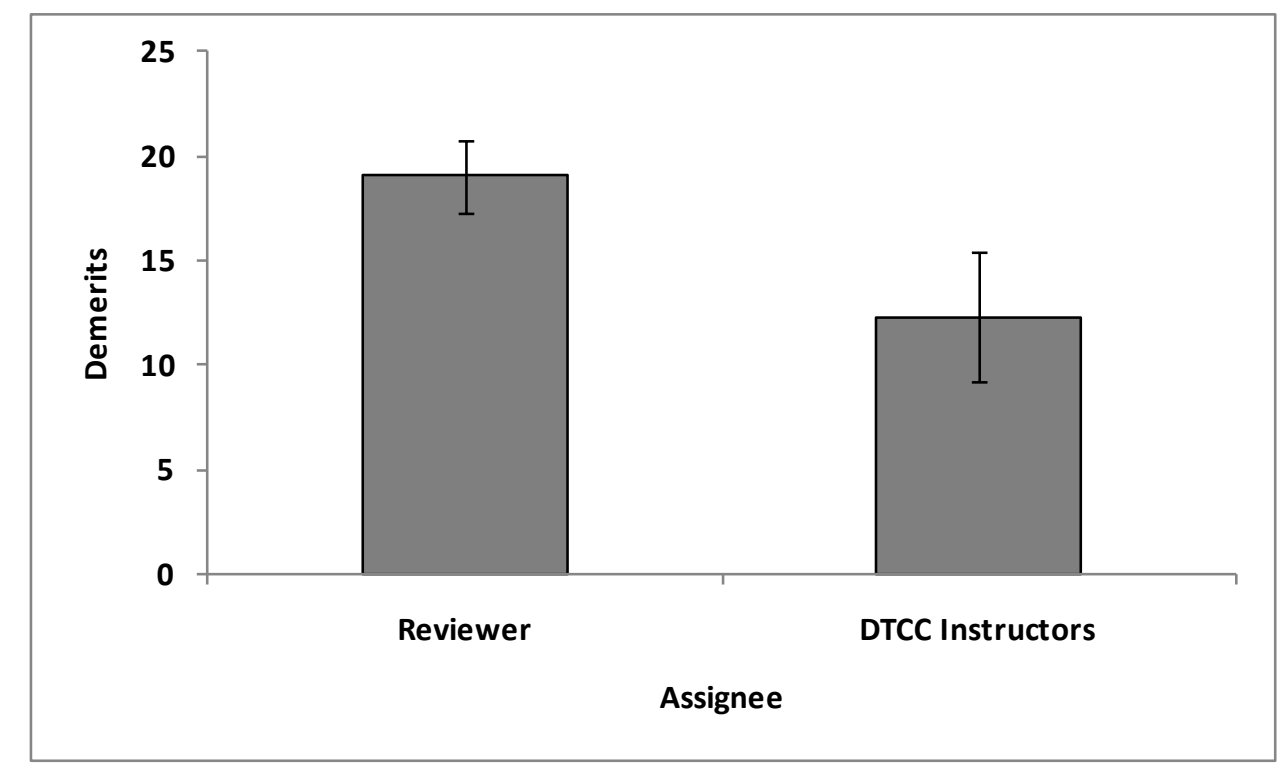

Figure 52. Chart. Demerits assigned to the Simulator Training Group, by rater. Error bars represent standard error.

Further analysis revealed three tests in the simulator training group with large differences between DTCC and reviewer demerits (Table 79). Two of these were Simulator road tests, and one was a DTCC road test. Two different DTCC instructors scored the three exams. The most likely cause for these discrepancies is the difference in perspective between the DTCC instructor (who is seated in the passenger seat of the vehicle) and the reviewer (who has views of the participant's face, forward roadway, and sides of the truck). 
Table 79. DTCC and Reviewer Discrepancies in Scoring

\begin{tabular}{|l|l|l|l|}
\hline Participant & \multicolumn{1}{|c|}{$\begin{array}{c}\text { Class } \\
\text { Session }\end{array}$} & \multicolumn{1}{|c|}{ Test } & \multicolumn{1}{c|}{ Discrepancy } \\
\hline 55 & 9 & DTCC & $\begin{array}{l}\text { DTCC identified 0 traffic check errors and one 5- } \\
\text { point error for a curb strike. Reviewer identified 9 } \\
\text { traffic check errors and two 5-point errors for curb } \\
\text { strikes. }\end{array}$ \\
\hline 61 & 3 & Simulator & $\begin{array}{l}\text { DTCC identified 0 traffic check errors. Reviewer } \\
\text { identified 17 traffic check errors. }\end{array}$ \\
\hline 67 & 14 & Simulator & $\begin{array}{l}\text { DTCC identified 0 traffic check errors. Reviewer } \\
\text { identified 7 traffic check errors. }\end{array}$ \\
\hline
\end{tabular}

As most discrepancies were from traffic checks, it is likely that the DTCC personnel were not able to clearly tell if the participant was performing a traffic check; in effect DTCC instructors appeared to give the benefit of the doubt to participants when they could not make a clear determination of head movement. This is because the DTCC instructors were seated behind the participant (in the case of Simulator testing) or in the passenger seat of the cabin (in the case of DTCC testing); both situations precluded the DTCC instructor having a clear view of the driver's face. However, the reviewer could always make a clear determination of head movement through viewing the participant's face directly on the video recording. The discrepancy in curb strikes could have arisen due to the perspective that the reviewer had of the wheels: the single camera view for each side of the vehicle was provided by a fender-mounted camera. If the strike occurred by a rear trailer wheel, the view might provide an ambiguous picture. This explanation is offered because it is assumed that the DTCC instructor riding in the vehicle would have felt a curb strike during real-vehicle testing.

The change scores were further analyzed for differences between DTCC instructor ratings and reviewer ratings by test mode (DTCC and Simulator tests). No significant differences were found $(p>.05)$.

\section{RANGE TEST VALIDATION}

A total of 72 range tests were examined by the reviewer. Descriptive statistics for DTCC demerits, reviewer demerits, and change scores are presented in Table 80. 


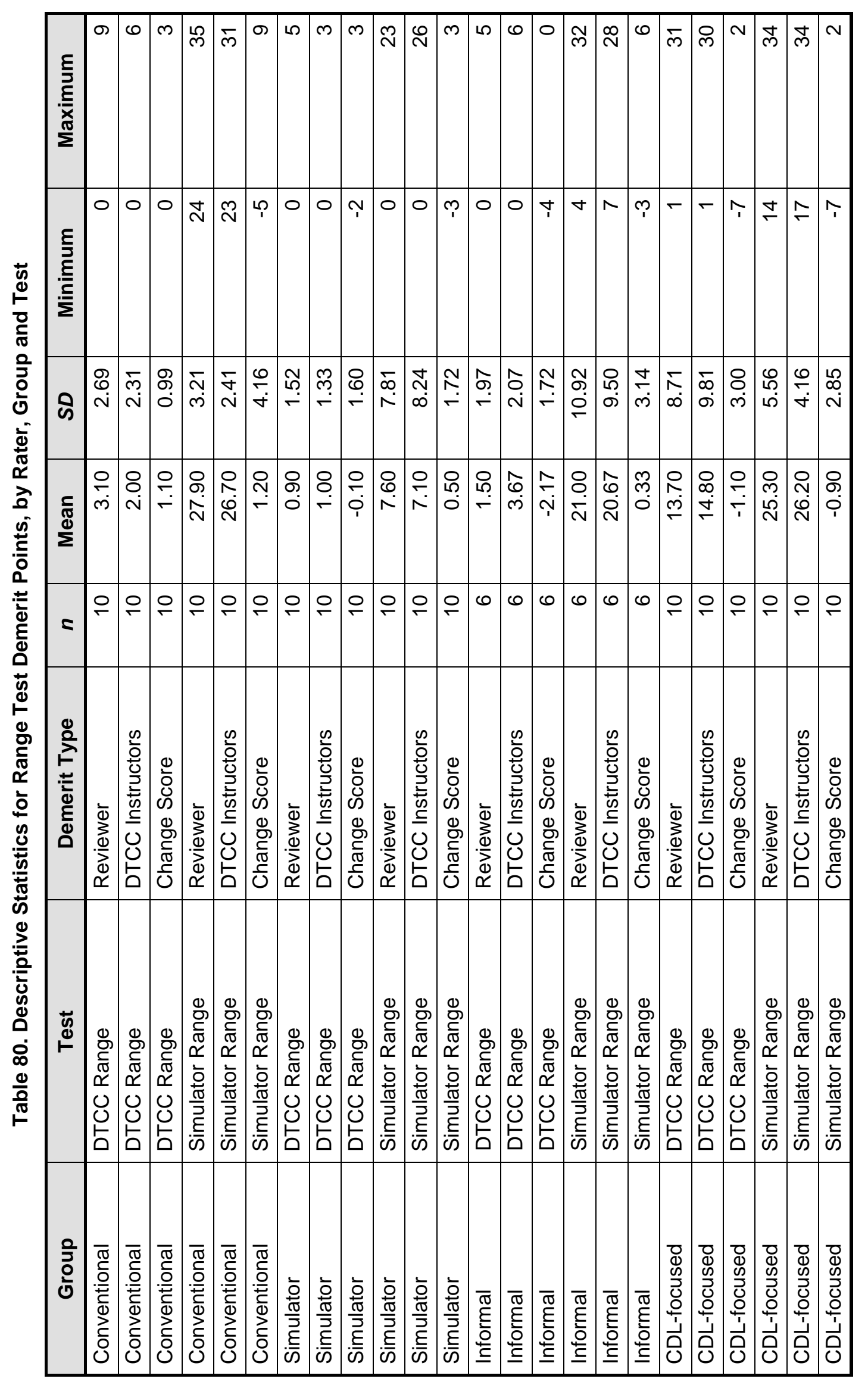


The change scores were analyzed for differences between DTCC instructor ratings and reviewer ratings by training group membership and test mode (DTCC and Simulator tests). In both cases, no significant differences were found ( $p>.05)$.

\section{DISCUSSION}

Reviewer- and DTCC-assigned demerit points were compared for both road and range tests via change scores. Analysis of the road tests indicated the presence of a significant difference between DTCC and reviewer scoring of the simulator training group's tests. The DTCC personnel assigned a lower number of demerit points to this group than the reviewer did; however, this difference was an average of 6.79 points. The majority of these demerit points were assigned for traffic checks; this presents a possible explanation for this discrepancy as the reviewer had a clear view of the participant's face while the DTCC instructor could only indirectly view the participant's head. No other group differences, and no test modality differences, were present. Analysis of the range tests indicated no significant differences in demerits assigned by the reviewer and DTCC personnel. 


\section{APPENDIX L-END-OF-TESTING PARTICIPANT ASSESSMENT}
Date:

\section{Commercial Driver End of
Participant \#:}
Group \#:

Please complete the following assessment of simulator performance, your driving safety skills, and your evaluation of the training that you received prior to becoming a commercial driver.

Part 1. Simulator Evaluation

Please compare the following features in the simulator to an actual tractor trailer and place an $\mathrm{X}$ along the scale that best represents your response.

Example:

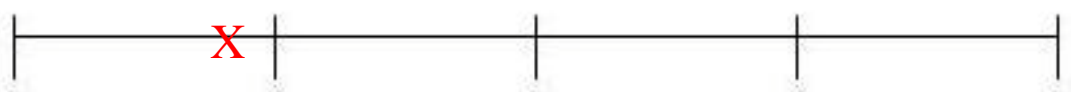

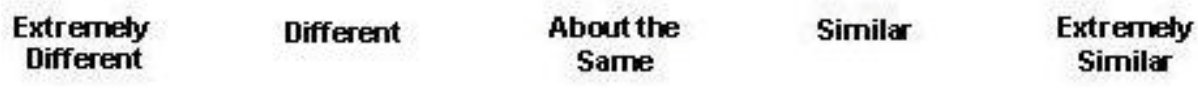

1. Upshifting:

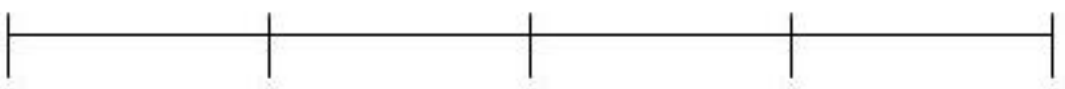

\begin{tabular}{|c|c|c|c|}
\hline $\begin{array}{l}\text { Extremely } \\
\text { Different }\end{array}$ & Different & $\begin{array}{l}\text { Abour the } \\
\text { Same }\end{array}$ & Similar \\
\hline
\end{tabular}

2. Downshifting:

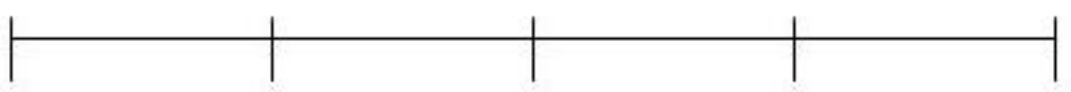

\begin{tabular}{ccccc|}
$\begin{array}{c}\text { Extremely } \\
\text { Different }\end{array}$ & Different & $\begin{array}{c}\text { About the } \\
\text { Same }\end{array}$ & Similar & $\begin{array}{c}\text { Extremely } \\
\text { Similar }\end{array}$ \\
\hline & & & & \\
\hline $\begin{array}{c}\text { Extremely } \\
\text { Different }\end{array}$ & Different & $\begin{array}{c}\text { Abourt the } \\
\text { Same }\end{array}$ & Similar & $\begin{array}{c}\text { Extremely } \\
\text { Similar }\end{array}$
\end{tabular}


4. Braking:

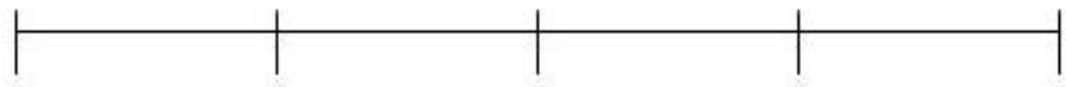

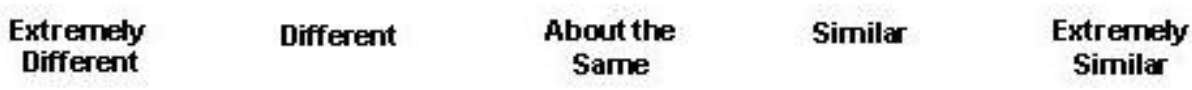

5. Steering:

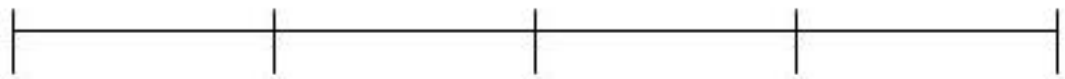

\begin{tabular}{|c|c|c|c|}
\hline $\begin{array}{l}\text { Extremely } \\
\text { Different }\end{array}$ & Different & $\begin{array}{c}\text { About the } \\
\text { Same }\end{array}$ & Similar \\
\hline
\end{tabular}

6. Turning:

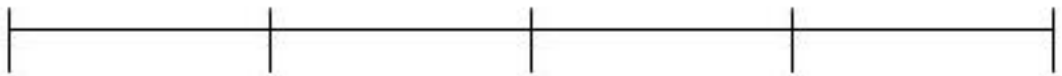

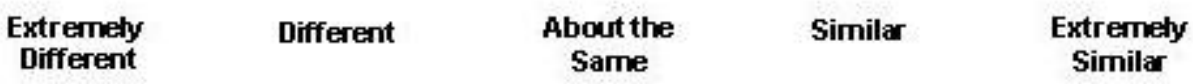

7. Handling

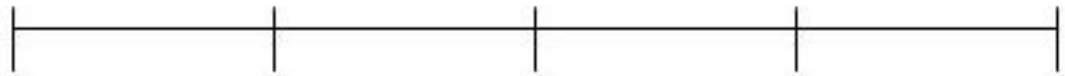

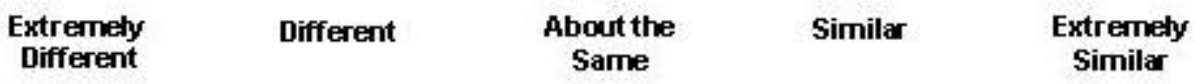

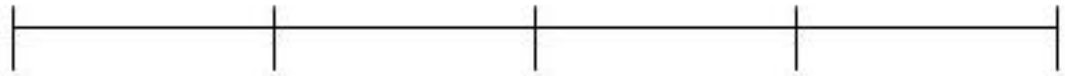

8. Mirrors:

(Views realistic)

Extremehy

Different

About the

Similar

Extremely

Different

Same

Similar

9. Trailer:

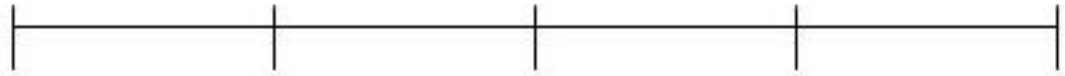

(Characteristics \& handling)

Different

About the

Similar

Extremely

Different

Same

Similar

10. Visuals:

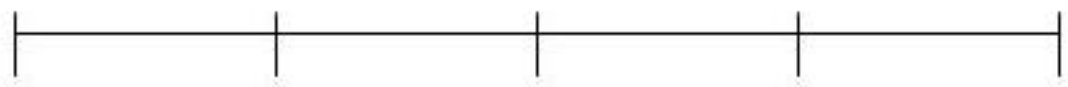

(Signs, lane

markings, road

Extremehy

Different
About the
Same

Similar

Extremely

surface, other Different

Similar 
11. Potential Threats:

(Situations you

encountered)

12. Overall Realism:

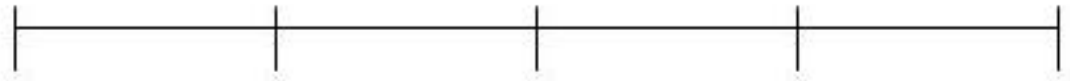

\begin{tabular}{|c|c|c|c|}
\hline $\begin{array}{l}\text { Extremely } \\
\text { Different }\end{array}$ & Different & $\begin{array}{l}\text { About the } \\
\text { Same }\end{array}$ & Sirnilar \\
\hline
\end{tabular}

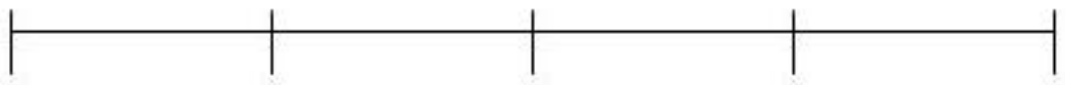

$\begin{array}{cccc}\begin{array}{c}\text { Extremely } \\ \text { Different }\end{array} \text { Different } & \begin{array}{c}\text { About the } \\ \text { Same }\end{array} & \text { Similar } & \begin{array}{c}\text { Extremely } \\ \text { Similar }\end{array}\end{array}$

13. Skills:

(How did you

handle the situations

Extremely

Different

About the

Similar

Extremeby

Different Same Similar

encountered in the

scenario?)

14. What aspect(s) of the simulator do you think were most effective? (Check all that apply and rank the top 3 among those with 1 being the most effective)

\begin{tabular}{|l|l|l|}
\hline \multicolumn{1}{|c|}{ Aspect } & $\begin{array}{c}\text { Most Effective } \\
\text { (check all that apply) }\end{array}$ & $\begin{array}{c}\text { Top 3 } \\
(\mathbf{1 , 2}, \mathbf{3})\end{array}$ \\
\hline Safety & & \\
\hline Overhead Views & & \\
\hline Repeatability & & \\
\hline Standardization & & \\
\hline Realism & & \\
\hline Enrichment (e.g., weather, mountains) & & \\
\hline Basic Control & & \\
\hline Vehicle Handling & & \\
\hline Safe Operations & & \\
\hline Backing Skills & & \\
\hline
\end{tabular}


15. What aspect(s) of the simulator do you think were least effective? (Check all that apply and rank the top 3 among those with 1 being the least effective)

\begin{tabular}{|l|c|c|}
\hline \multicolumn{1}{|c|}{ Aspect } & $\begin{array}{c}\text { Least Effective } \\
\text { (check all that apply) }\end{array}$ & $\begin{array}{c}\text { Top 3 } \\
(\mathbf{1}, \mathbf{2}, \mathbf{3})\end{array}$ \\
\hline Safety & & \\
\hline Overhead Views & & \\
\hline Repeatability & & \\
\hline Standardization & & \\
\hline Realism & & \\
\hline Enrichment (e.g., weather, mountains) & & \\
\hline Basic Control & & \\
\hline Vehicle Handling & & \\
\hline Safe Operations & & \\
\hline Backing Skills & & \\
\hline
\end{tabular}

\section{Part 2. General Evaluation}

Please answer each of the following questions by placing an $\mathrm{X}$ along the scale that best represents your response.

Example:
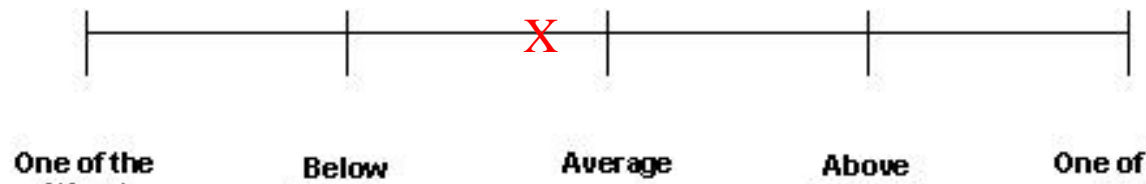

Worst

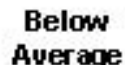

Average

Above

Average

One of the

Best

1. Compared to other drivers with equal experience, how would you rate your current vehicle control and maneuvering skills (e.g., proper shifting, smooth acceleration \& braking)?

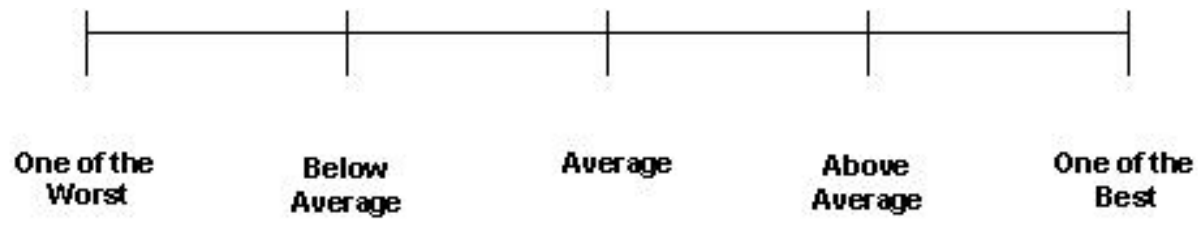

2. Compared to other drivers with equal experience, how would you rate your current safe operations skills (e.g., defensive driving, speed maintenance, space management)?

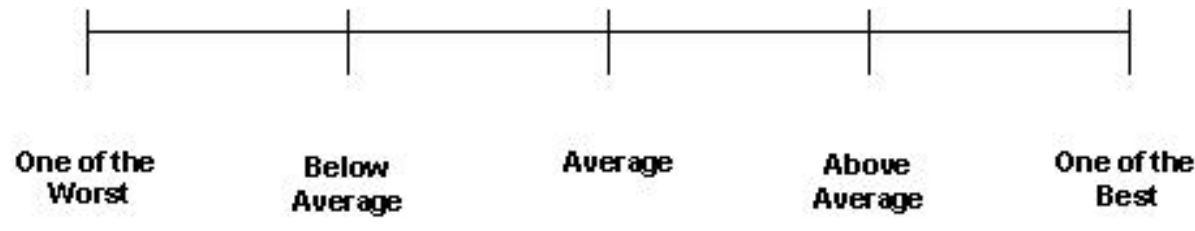


3. How would you rate the entry-level training you received in terms of teaching you vehicle control and maneuvering skills (e.g., proper shifting, smooth acceleration \& braking)?

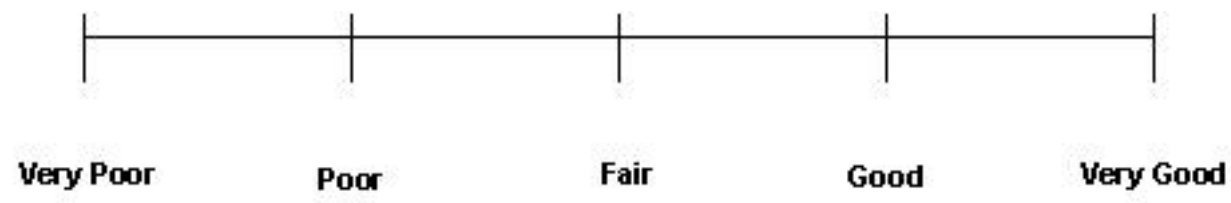

4. Looking back, how would you rate the entry-level training you received in terms of teaching you safe operations (e.g., defensive driving skills, speed ,maintenance, space management)?
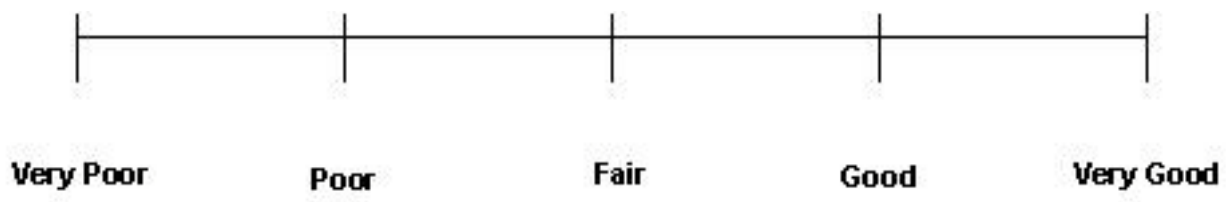

5. How do you think the amount of behind-the-wheel training you received compares to other drivers?

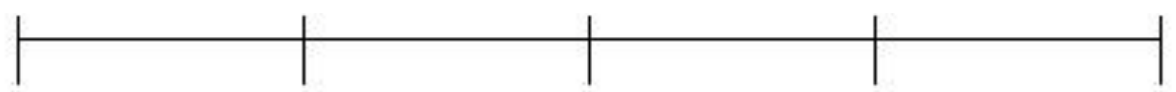

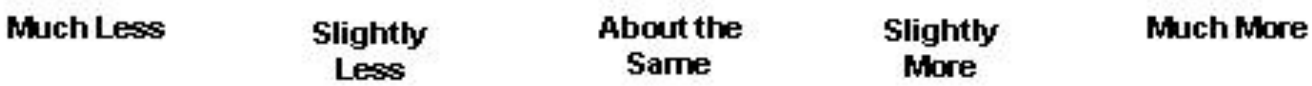

Why?

6. Would you recommend the training you received to others? (Select one)

No Yes

Why?

Next page please. 
Part 3. Skills Tests Evaluation

Please answer each of the following questions by placing an $\mathrm{X}$ along the scale that best represents your response. As part of the study, your skills were tested on a simulator and a tractor trailer. Do you think that these tests were a fair assessment of your driving skills at the time? (Circle one per test)

1. Simulated Skills Test:

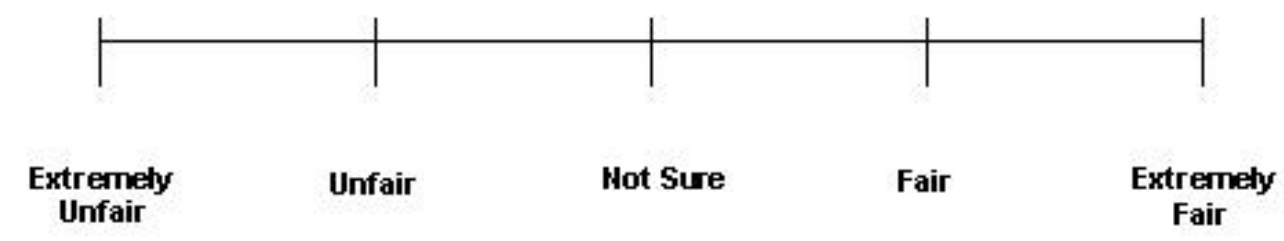

2. Behind-the-Wheel DTCC Skills Test:

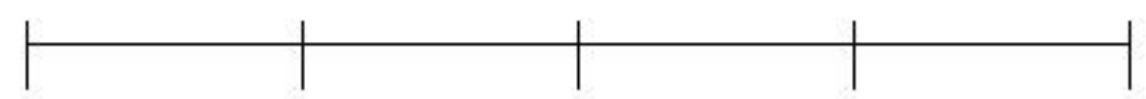

Unfair

Hot Sure

Fair

Extremely

Unfair 


\section{APPENDIX M-SIMULATOR CONFIGURATIONS CURRENTLY IN USE}

As simulator technology has evolved over the years, the number of trucking companies and training facilities employing the use of CMV simulators to provide training to their drivers has grown. These simulators are being used to enhance and supplement driver training. Table 81 below identifies trucking entities currently employing CMV simulators and which vendor and model they are using. The three vendors identified in Table 81 were contacted to obtain the pricing and specifications for these simulators as well as any additional simulator models manufactured by these vendors. It should be noted that Lockheed Martin is no longer manufacturing CMV simulators, and the L3 TransSim VS IV has replaced the TransSim VS III. One Doron Precision simulator was identified as being in use (the model 460Truck, price quoted at $\$ 203,695)$. Doron Precision also manufactures a second line of CMV simulators, the 550Truck at a cost of $\$ 120,000$. It should be noted that simulators can be customized based on customer needs, drastically increasing their cost. Table 81 and Table 82 provide a broad scope of simulators currently in use and/or are available in the current market.

Table 81. Simulators Identified as In-Use within the Industry

\begin{tabular}{|l|l|r|r|}
\hline \multicolumn{1}{|c|}{$\begin{array}{c}\text { Simulator } \\
\text { Manufacturer }\end{array}$} & \multicolumn{1}{|c|}{ Simulator Model and Configuration } \\
Notes & $\begin{array}{c}\text { Simulator Model } \\
\text { Base Cost } \\
\text { Approximate) }\end{array}$ & $\begin{array}{c}\text { Number of } \\
\text { Simulators } \\
\text { in Use }\end{array}$ \\
\hline Doron Precision & 460Truck & $\$ 203,695$ & 1 \\
\hline FAAC & TT-1000-V1 & $\$ 105,000$ & 1 \\
\hline FAAC & TT-1000-V3 & $\$ 120,000$ & 3 \\
\hline FAAC & TT-2000-V3 & $\$ 500,000$ & 1 \\
\hline FAAC & TT-2000-V5 (6-DOF Freightliner cab) & $\$ 290,000$ & 1 \\
\hline FAAC & TT-2000-V7 (3-DOF generic cab) & $\$ 350,000$ & 2 \\
\hline L3 & Mark III & $\mathrm{N} / \mathrm{A}$ & 4 \\
\hline L3 & TransSim VS III & $\$ 125,000$ & 3 \\
\hline L3 & TransSim VS IV & $\mathrm{N} / \mathrm{A}$ & 1 \\
\hline Lockheed Martin & MDTS (Frieghtliner cab) & \\
\hline
\end{tabular}

Table 82 describes the basic simulator specifications for each of the simulators presented above. It should be noted that Lockheed Martin has been omitted from this table as they have ceased manufacturing CMV simulators. Additionally, Doron Precision Systems has been included. 


\begin{tabular}{|c|c|c|c|c|c|c|c|c|c|c|}
\hline 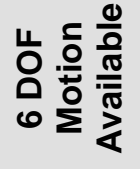 & 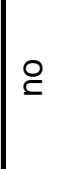 & $\stackrel{9}{\cong}$ & $\stackrel{8}{9}$ & $\stackrel{9}{\complement}$ & $\stackrel{\infty}{\stackrel{\infty}{\infty}}$ & $\stackrel{0}{0}$ & ㅇ & 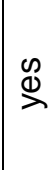 & $\stackrel{9}{\complement}$ & 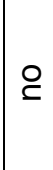 \\
\hline 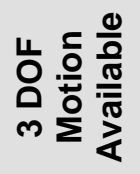 & 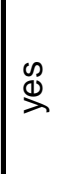 & $\stackrel{\infty}{\rightleftharpoons}$ & $\stackrel{\varrho}{\stackrel{\varrho}{\nu}}$ & 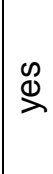 & $\stackrel{\mathscr{U}}{\stackrel{\mathscr{U}}{\lambda}}$ & $\stackrel{\mathscr{Q}}{\nu}$ & ㅇ & 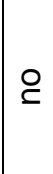 & 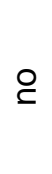 & 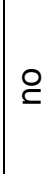 \\
\hline 厄๊ & \begin{tabular}{|c}
$\overline{\mathrm{d}}$ \\
응
\end{tabular} & 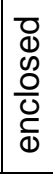 & ळे & 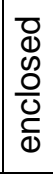 & 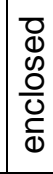 & $\begin{array}{l}D \\
d \\
0 \\
0 \\
0 \\
0 \\
\\
\end{array}$ & 흥 & 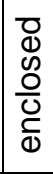 & $\begin{array}{l}\frac{c}{\Phi} \\
\frac{0}{0}\end{array}$ & 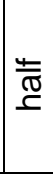 \\
\hline 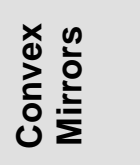 & 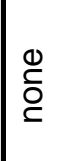 & 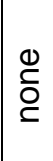 & $\frac{\bar{\sigma}}{\stackrel{3}{*}}$ & $\stackrel{\bar{\sigma}}{\frac{\pi}{2}}$ & $\frac{\bar{\pi}}{\stackrel{3}{\frac{\pi}{\gamma}}}$ & $\frac{\bar{\sigma}}{2}$ & 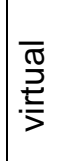 & 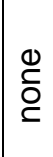 & $\frac{\sqrt[\sigma]{\underline{N}}}{\bar{y}}$ & $\frac{\bar{\sigma}}{\overrightarrow{3}}$ \\
\hline 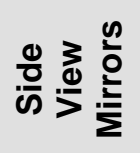 & \begin{tabular}{|l}
$\stackrel{0}{0}$ \\
$\stackrel{0}{\Xi}$ \\
\end{tabular} & 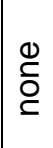 & 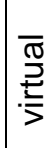 & 氶 & 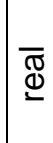 & 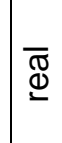 & 嵒 & ن & 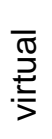 & 哥 \\
\hline 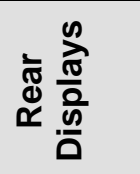 & 0 & 0 & 0 & 0 & $N$ & $\sim$ & 0 & 0 & 0 & 0 \\
\hline 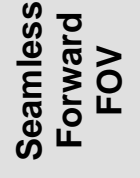 & 2 & zo & z & 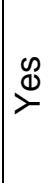 & $\underset{\nu}{\mathscr{U}}$ & $\stackrel{\mathscr{d}}{\succ}$ & z & 운 & z & 은 \\
\hline 인 & $\stackrel{12}{1}$ & $\stackrel{10}{1}$ & $\underset{\sim}{\infty}$ & ণิ & 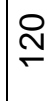 & $\stackrel{\mathscr{N}}{N}$ & 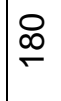 & 啰 & $\underset{\sim}{\stackrel{\leftrightarrow}{\rightarrow ~}}$ & 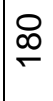 \\
\hline 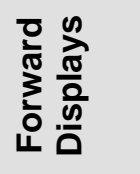 & -1 & -1 & $m$ & $m$ & ما & $\wedge$ & m & m & $m$ & $\nabla$ \\
\hline $\begin{array}{l}\bar{\Phi} \\
\frac{0}{2}\end{array}$ & $\mid \begin{array}{c}5 \\
\vdots \\
0 \\
0 \\
1 \\
1 \\
1\end{array}$ & 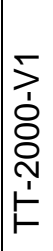 & $\begin{array}{l}m \\
0 \\
8 \\
8 \\
7 \\
1 \\
1\end{array}$ & 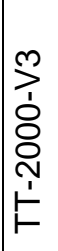 & 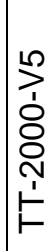 & $\begin{array}{l}1 \\
\dot{1} \\
\delta \\
0 \\
N \\
1\end{array}$ & 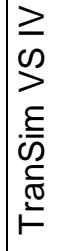 & $\begin{array}{l}\equiv \\
\text { 立 } \\
\overline{\bar{\nu}} \\
\Sigma\end{array}$ & 总 & 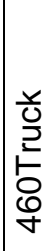 \\
\hline 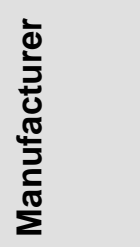 & $\mid \begin{array}{l}0 \\
\\
4 \\
4 \\
4\end{array}$ & $\mid \begin{array}{l}0 \\
\frac{\alpha}{4} \\
4\end{array}$ & $\begin{array}{l}0 \\
\frac{1}{4} \\
4\end{array}$ & $\begin{array}{l}0 \\
\end{array}$ & 晏 & 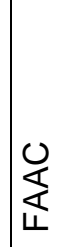 & m & $m$ & هั & ठั \\
\hline
\end{tabular}




\section{APPENDIX N—CASE STUDY FOCUS GROUP MEETING PROTOCOL}

\section{Greetings and Informed Consent (10 minutes)}

- Participants will be greeted and escorted to the conference room.

- An investigator will check each participant's CDL to verify that it is valid/current.

- Participants will be given the Informed Consent Form and asked to read the form and voice any concerns or questions that they have to an investigator.

- If subjects wish to participate, they will be asked to sign the last page of the Informed Consent Form. One form will be collected by the experimenter and the second will be given to the participant to keep for their own records.

- Those who choose not to participate may leave.

- Participants will be reminded that they may refuse to answer any questions and may leave at anytime.

\section{Facilitator Introduction and Ground Rules (5 minutes)}

Hello, my name is . We want to thank you for taking the time (today/this morning/this evening) to come and share your thoughts and opinions with us.

\section{PURPOSE OF THE MEETING}

- The purpose of this meeting is to discuss issues related to truck simulator driving programs.

- We are going to ask you a series of questions and need you to respond as openly and honestly as possible There are no right or wrong answers-we just want your opinions.

\section{CONFIDENTIALITY}

- The discussion tonight is strictly for research purposes, we are not selling anything and we will not connect anything you say with your name.

- There is a tape recorder in the room. Please speak loudly and clearly so that we get a good recording of your comments. 
- There will be a transcript of this discussion, but it will not match comments with names.

- Recordings will be stored in a secure location and erased after they are transcribed.

- If you feel uncomfortable, you can refuse to answer a question or you may leave.

- Did everyone fill out one of these consent forms? Do you have any questions about it?

\section{LOGISTICS}

- This meeting will run until (time), we are very appreciative of the time that you are spending and will honor it by not running over.

- Bathrooms are located around the corner to your left. If you would like something to eat or drink please help yourself now before we get started. We will also have a break later.

- Please turn off any phones/beepers unless you need them on for emergency reasons. This will help us to avoid distractions and finish on time.

\section{GROUND RULES}

- Please let me know if you are uncomfortable with any of these rules. If you are ok with these rules, then let's agree to abide by them for the remainder of the meeting.

- Avoid names, nicknames, or other identifying information

- Listen to each other

- Everyone participate fully

- No side conversations

- Spelling does not count

- Don’t criticize others

- Finish on time

- Return from break in a timely manner 


\section{Facilitator Questions and Discussion Rules (40 to 60 minutes)}

Note. These are primary questions (probes). Additional, in-depth, follow up questions may be used and will depend on the responses given to these primary questions. These follow up questions will be directly related to the general line of questions presented herein.

\section{Introduction/Simulators}

1. How is the truck simulator different than real-world driving?

2. How is the truck simulator similar to real-world driving?

3. What are the benefits of the truck simulator?

4. What are the disadvantages of using the truck simulator?

5. Do you believe the information you have learned in the truck simulator will transfer to real-world driving?

6. How important is a realistic simulation to learning on the truck simulator?

7. How would you change the truck simulator?

\section{Simulators in Training}

1. What is the truck simulator's role in the driver training program? (How is the truck simulator integrated in the driver training program?)

2. How is training in a truck simulator different than training in a real vehicle?

3. How is training in a truck simulator similar to training in a real vehicle?

4. Does training in a truck simulator change how you are taught?

5. Who would benefit most from a truck simulator training program?

6. If you were in charge of the truck simulator training program, what would you change? (What are some ways the truck simulator training program can be improved?)

7. If you were in charge of the truck simulator training program, what would be the top thing you would keep? (What are some ways the truck simulator training program is doing well?)

8. How could a truck simulator be used differently in your driver training program?

9. Overall, how would you rate the truck simulator training program? 



\section{ACKNOWLEDGEMENTS}

The authors of this report wish to thank the instructors and administration of Delaware Technical \& Community College for their continued support throughout this research study. Additional gratitude goes out to the DTCC Commercial Transportation Program instructors, Chris Antonik, Lee Derrickson, Jack Taylor, Glenn Kotch, and Bob Grover, for all of their dedicated hard work, advice, and daily support.

The authors wish to thank Mark Johnson, Robert McClanahan, John McFann, Jim McKnight, Gerald Krueger, and Kevin Lewis for their valuable advice, support, feedback, and technical assistance with this project.

The authors wish to thank the Delaware Division of Motor Vehicles and the Professional Truck Driver Institute for all of their assistance and comments, as well as the companies that provided assistance in the recruitment of study participants: Perdue Transportation Inc., Mountaire, PepUp Fuel, FedEx, Con-Way, Walmart, and Berry Van Lines. 



\section{REFERENCES}

American Transportation Research Institute (ATRI). (2005) Predicting Truck Crash Involvement, Developing of a Crash Behavior Based Model and Recommended Countermeasures. Arlington, VA: American Transportation Research Institute,.

American Transportation Research Institute (ATRI). (2008) A technical analysis of driver training impact on safety. Arlington, VA: American Transportation Research Institute.

Antonik, C., \& Derrickson, L. (1999). DTCC commercial transportation program entry level training curriculum. Georgetown, Delaware: DTCC.

Blanco, M., Hickman, J.S., Klauer, S.G., \& Hanowski, R.J. (2006). The new generation human factors naturalistic driving research: A proactive approach to enhance safety. In Proceedings of the 6th Annual Intelligent Vehicle Systems Symposium. National Defense Industrial Association.

Brock, J.F., Jacobs, C., Van Cott, H., McCauley, M.E., \& Norstrom, D.M. (2001). Simulators and bus safety: Guidelines for acquiring and using transit bus operator driving simulators. (Transit Cooperative Research Program, Report 72). Washington, DC.: Transportation Research Board.

Brock, J.F., McFann J., Inderbitzen, R., \& Bergoffen, G., (2007). Effectiveness of Commercial Motor vehicle driver training curricula and delivery methods (Commercial Truck and Bus Safety Synthesis 13). Washington, DC.: Transportation Research Board.

Bureau of Labor Statistics. (2008, May). Occupation employment and wages: 53-3032 Truck drivers, heavy and tractor-trailer. Retrieved February 10, 2010 from www.bls.gov/oes/current/oes533032.htm. Washington, DC: Bureau of Labor Statistics.

Bureau of Labor Statistics. (2009). Occupational outlook handbook, 2010-11 edition: Truck drivers and driver/sales workers. Retrieved February 10, 2010, from www.bls.gov/oco/ocos246.htm. Washington, DC: Bureau of Labor Statistics.

Carroll, R.J., \& Dueker, R.L. (1996). Commercial Motor Vehicle Simulation Technology to Improve Driver Training, Testing, and Licensing Methods (Report No. FHWA-MC-96-003). Washington, DC: USDOT, FHWA Office of Motor Carriers.

Dueker, R. L. (1995). Assessing the Adequacy of Commercial Motor Vehicle Driver Training: Final Report. (Publication No. FHWA-MC-96-011). Washington, DC: USDOT, FHWA, Office of Motor Carriers.

Dugan, R.T. (2008, October 6). Training, turnover. [Letter to the editor]. Transportation Topics, p. 9.

Emery, C., Robin, J., Knipling, R., Finn, R., \& Fleger, S. (1999). Research design: Validation of simulation technology in the training, testing, and licensing of tractor-trailer drivers: Final Report (Report No. FHWA-MC-99-060). Washington, DC: USDOT, FHWA, Office of Motor Carrier and Highway Safety.

Ericksson, K., \& Simon, H. (1980). Verbal reports as data. Psychological Review, 87(3), 215251. 
Federal Motor Carrier Safety Administration (2007, December). Notice of Proposed Rulemaking Minimum Training Requirements for Entry-Level Commercial Motor Vehicle Operators [Docket No. FMCSA-2007-27748 RIN 2126-AB06]. Washington, DC, Office of the Federal Registrar.

Goldberg, L.R.(1992). The development of markers for the Big-Five factor structure . Psychological Assessment, 4, 26-42.

Golding, J.F. (2006). Predicting individual differences in motion sickness susceptibility by questionnaire. Personality and Individual Differences, 41, 237-248.

Hartman, K., Pritchard, R., Jennings, K., Johnston, J., Knipling, R.R., et al., (2000). Commercial Vehicle Safety - Technology and Practice in Europe. (Document no. FHWA-PL-00-010) Washington, DC: USDOT, FHWA, Office of International Programs.

Howard, J., Zuckerman, A., Strah, T.M., \& McNally, S. (2009, February 16). Trucking’s growing job losses. Transport Topics, pp. 6.

Hughes, R (2001). The Effectiveness of Commercial Motor Vehicle (CMV) Enforcement in Reducing Truck-involved Crashes. Chapel Hill, NC: UNC Chapel Hill Highway Safety Research Center.

Juang, M., \& Alessi, N.E. (2000). An introduction to virtual reality in psychiatry. Canadian Psychiatric Association Bulletin, 32, 1-3.

Kennedy R.S., Fowlkes, J.E., Berbaum K.S., \& Lilienthal M.G. (1992). Use of a motion sickness history questionnaire for prediction of simulator sickness. Aviation, Space, and Environmental Medicine, 63(7), 588-593.

Kennedy, R.S., Lane, N.E., Berbaum, K.S., \& Lilienthal, M.G. (1993). Simulator sickness questionnaire: an enhanced method for quantifying simulator sickness. International Journal of Aviation Psychology, 3(3), 203-220.

Kneebone, R.L., Nestel, D., Vincent, C., \& Darzi, A. (2007). Complexity, risk and simulation in learning procedural skills. Medical Education, 41, 808-814.

Knipling, R.R., Hickman, J.S., \& Bergoffen, G. (2003). Effective Commercial Truck and Bus Safety Management Techniques. (Commercial Truck and Bus Safety Synthesis Program, Synthesis 1). Washington, DC: Transportation Research Board.

Lantz B., \& Blevins, M. (2001). An analysis of commercial vehicle driver traffic conviction data to identify high safety risk motor carriers. Fargo, ND: Upper Great Plains Transportation Institute.

Murray, D., Shackelford, S., Houser, A. (2009). Analysis and Benefits of Cost of Roll Stability Control System for the Trucking Industry (Publication No. FMCSA RT-09-020). Washington, DC: USDOT, FMCSA.

Office of Management and Budget. (1992). Guidelines and Discount Rates for Benefit-Cost Analysis of Federal Programs. (OMB Circular A-94). Washington DC: Executive Office of the President. 
Orlansky, J., \& String, J. (1977). Cost-effectiveness of flight simulator for military training. Use and Electiveness of Flight Simulators: Vol. 1 (Report No. P-1275). Arlington, VA: Institute for Defense Analysis.

Parkes, A.M. (2003). Truck driver training using simulation in England. Proceedings of the Second International Driving Symposium on Human Factors in Driver Assessment, Training and Vehicle Design (pp. 59-63). Iowa City: University of Iowa.

Pausch, R., Crea, T., \& Conway, M. (1992). A literature survey for virtual environments: military flight simulator visual systems and simulator sickness. Presence: Teleoperators and Virtual Environments, 1, 344-363.

Pierowicz, J., Robin, J., Gawron, V., Watson, G., Nestor, B., \& Murphree, W. (2002). Commercial truck simulators re-assessment and evaluation. (Report No. FMCSA RT-03008). Washington, DC: USDOT, FMCSA Office of Research and Technology.

Professional Truck Driver Institute. (1999, September). Curriculum standard guidelines for entry-level tractor-trailer driver courses. Alexandria, VA: Author.

Rasmussen, J. (1983). Skills, rules, and knowledge: Signals, signs, and symbols, and other distinctions in human performance models. IEEE Transactions on Systems, Man, and Cybernetics, SMC-13(3), 257-266.

Reason, J.T. (2008). The human contribution: unsafe acts, accidents and heroic recoveries. Surrey, UK: Ashgate.

Riva, G., \& Gamberlini, L. (2000). Virtual reality in telemedicine. CyberPsychology and Behavior, 5, 219-224.

Robin, J.L., Knipling, R.R., Derrickson, M.L, Antonik, C., Tidwell, S.A., \& McFann, J. (2005b). Truck simulator validation ("SimVal") training effectiveness study. Proceedings of the 2005 Truck \& Bus Safety \& Security Symposium (pp. 475-483). Alexandria, VA: National Safety Council.

Robin, J.L., Knipling, R.R., Tidwell, S.A., McFann, J., Derrickson, M.L., \& Antonik, C. (2005a). FMCSA Commercial Truck Simulation Validation Study Phase I Pilot Test: Driving Scenario Definition and Development, Driving Simulation Conference North America 2005. Orlando, FL.

State of Delaware Division of Motor Vehicles. (2005). State of Delaware Commercial Driver License Manual, Version 2.0. Wilmington, Delaware: Author.

Stock, D. (2001). I-95 Corridor Coalition Field Operational Test 10: Coordinated Safety Management; Volume I: Best Practices in Motor Carrier Safety Management, Final Report. I-95 Corridor Coalition: Rockville, MD.

US Air Force. (2008). Link Trainer Fact Sheet. Retrieved 15 October 2008, from http://www.nationalmuseum.af.mil/factsheets/factsheet_print.asp?fsID=3371\&page=1.

Wachtel, J. (1995). Brief history of driving simulators. TR News, 45, $26-27$.

Willis, J. (2009) Office Trends Report Trends Report Third Quarter 2009. Charleston, SC: Grubb \& Ellis. 
Witmer, B.G., \& Singer, M.J. (1998). Measuring presence in virtual environments: A presence questionnaire. Presence: Teleoperators and Virtual Environments, 7, 225-240.

Woodward, M. (2005). Epidemiology: Study design and data analysis (2nd Ed.). Boca Raton, FL: CRC Press 